Florida International University

FIU Digital Commons

FIU Electronic Theses and Dissertations

University Graduate School

10-23-2019

\title{
Graphene Foam Reinforced Shape Memory Polymer Epoxy Composites
}

Adeyinka Idowu

aidow002@fiu.edu

Follow this and additional works at: https://digitalcommons.fiu.edu/etd

Part of the Nanoscience and Nanotechnology Commons, Polymer and Organic Materials Commons, and the Structural Materials Commons

\section{Recommended Citation}

Idowu, Adeyinka, "Graphene Foam Reinforced Shape Memory Polymer Epoxy Composites" (2019). FIU Electronic Theses and Dissertations. 4350.

https://digitalcommons.fiu.edu/etd/4350

This work is brought to you for free and open access by the University Graduate School at FIU Digital Commons. It has been accepted for inclusion in FIU Electronic Theses and Dissertations by an authorized administrator of FIU Digital Commons. For more information, please contact dcc@fiu.edu. 


\section{FLORIDA INTERNATIONAL UNIVERSITY}

Miami, Florida

GRAPHENE FOAM-REINFORCED

\section{SHAPE MEMORY POLYMER EPOXY COMPOSITES}

A dissertation submitted in partial fulfillment of the

requirements for the degree of

DOCTOR OF PHILOSOPHY

in

MATERIALS SCIENCE AND ENGINEERING

by

Adeyinka Taiwo Idowu 


\section{To: Dean John Volakis}

College of Engineering and Computing

This dissertation, written by Adeyinka Taiwo Idowu, and entitled Graphene FoamReinforced Shape Memory Polymer Epoxy Composite, having been approved in respect to style and intellectual content, is referred to you for judgment.

We have read this dissertation and recommend that it be approved.

$\begin{array}{r}\text { Sharan Ramaswamy } \\ \hline \text { Norman Munroe } \\ \hline \text { Benjamin Boesl, Co-Major Professor } \\ \hline \text { Arvind Agarwal, Co-Major Professor }\end{array}$

Date of Defense: October 23, 2019

The dissertation of Adeyinka Taiwo Idowu is approved.

Dean John Volakis College of Engineering and Computing

Andrés G. Gil

Vice President for Research and Economic Development and Dean of the University Graduate School

Florida International University, 2019 
(C) Copyright 2019 by Adeyinka Taiwo Idowu

All rights reserved. 


\section{DEDICATION}

I dedicate this thesis first to the Almighty, All-Knowing God for empowering me to complete my $\mathrm{PhD}$ project, to my wife for standing by me through the thick and thin, to my parents for always believing in me and to my twin brother, elder brother and sister for inspiring me to pursue my dreams to fulfillment. 


\section{ACKNOWLEDGMENTS}

I sincerely and deeply thank my major advisor, Dr. Arvind Agarwal, for his inspiring guidance and invaluable support throughout the period of my doctoral study at Florida International University. Your motivating words to start and timely conclude my $\mathrm{PhD}$ journey are one of the forms of inspiration which has driven me to the conclusion of obtaining my terminal degree.

Special thanks to my co-major adviser, Dr. Benjamin Boesl, for your counsel and instilling in me the believe of executing the doctoral project. Your readiness to share your technical knowledge helped my line of thought while engaged in my $\mathrm{PhD}$ pursuit. I would like to thank my committee members, Dr. Norman Munroe and Dr. Sharan Ramaswamy, for giving great suggestions during my doctoral research.

I would like to acknowledge the supports from Plasma Forming Lab research group members. Your interesting interactions with me created positive atmosphere for me to conduct my PhD study. Particularly, I am thankful to Drs. Cheng Zhang and Tony Thomas for awesome technical discussion and assistance as senior members fo the group. My thanks go to Mr. Pranjal Nautiyal for technical discussion I had with you. I appreciate Dr. Archana Loganathan for her willingness to support me and create positive energy around me.

My appreciation goes to the support of the Advanced Materials Engineering Research Institute (AMERI) at FIU for providing research facilities. I also thank Dr. Alex Franco for the training and assistance he rendered at any time I am in AMERI. I am thankful to University Graduate School, FIU for supporting me through Dissertation Year Fellowship (DYF) award. 


\section{ABSTRACT OF THE DISSERTATION \\ GRAPHENE FOAM-REINFORCED \\ SHAPE MEMORY POLYMER EPOXY COMPOSITES}

by

Adeyinka Taiwo Idowu

Florida International University, 2019

Miami Florida

Professor Arvind Agarwal, Co-Major Professor

Professor Benjamin Boesl, Co-Major Professor

Shape memory polymer (SMP) epoxy has received growing interest due to its facile processing, low density, and high recoverable strain. Despite these positive attributes, SMP epoxy has drawbacks such as slow recovery rate, and inferior mechanical properties. The slow recovery rate restricts the use of SMP epoxy as a functional structure. The aim of the present work is to explore the capabilities of three-dimensional (3D) graphene foam $(\mathrm{GrF})$ and graphene nanoplatelet (GNP) as reinforcements in SMP epoxy to overcome their slow recovery and improve the mechanical properties. GrF and GNP based SMP epoxy composites are fabricated by mold-casting approach and 3D printing techniques, respectively. They are investigated for their thermal, shape recovery, and mechanical behaviors. 0.13 wt. $\%$ GrF addition results in $19 \%$ increase in the glass transition temperature (Tg) of mold-cast SMP epoxy. GrF-based SMP epoxy composite displays thermal conductivity of $0.296 \mathrm{~W} \mathrm{mk}^{-1}$ at $70^{\circ} \mathrm{C}$, which is $57 \%$ greater than that of SMP epoxy. The addition of GrF results in excellent thermal and electrical conductivity of SMP epoxy by providing a continuous network of graphene for phonon and electron flow, 
respectively. Thus, thermal and electrical stimulation are employed to actuate shape recovery in GrF-reinforced SMP epoxy composite. Maximum shape recovery ratio is achieved for thermally actuated GrF-based SMP epoxy composite with a $23 \%$ improvement in the recovery rate. GrF addition transforms a non-electrically conductive SMP epoxy to an electrically conductive polymer. Moreover, 0.5 wt.\% GrF integration enhances tensile strength and elastic modulus of SMP epoxy by $\sim 6 \%$ and $20 \%$, respectively which is attributed to excellent stress transfer from matrix to $\mathrm{GrF}$ reinforcement. Damping behavior of SMP-0.5 wt.\% GrF epoxy is also improved by $180 \%$. SMP epoxy-GNP composite is successfully 3D printed using a slurry-based extrusion technique. 3D printed composites exhibit complete shape recovery. A mere 0.1 wt.\% GNP addition resulted in enhanced tensile strength (30\%) and elastic modulus (17\%) than that of SMP epoxy. Damping behavior of 3D printed of SMP epoxy-GNP composite is also improved by $\sim 50 \%$ (below its $\mathrm{Tg}$ ) as compared to 3D printed SMP epoxy. This study demonstrates that graphene-based reinforcements endow SMP epoxy with multifunctional capabilities; thereby paving the way for a new generation of advanced shape memory polymer composite, finding potential applications in electro-mechanical systems, micro-robots and morphing wing of an aircraft. 
Chapter I: Introduction

1.1 Shape Memory Polymers and Their Applications - Advantages, Drawbacks and Solution. 1

1.2 Graphene Reinforcement - Its Capabilities to Shape Memory Polymers ...... 3

1.2.1 Challenges Associated with Graphene Reinforcement ......................... 4

1.3 3D Graphene Foam: Suitable reinforcement to shape memory polymer

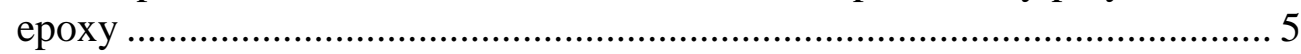

1.4 Objectives of Current Research .................................................................... 6

Chapter II: LITERATURE REVIEW …………………….............................. 8

2.1 Graphene-Based Polymer Composites ........................................................ 8

2.1.1 Interfacial interactions between graphene filler and polymers............. 8

2.1.2 Dispersion of graphene within the polymers ....................................... 9

2.1.3 High inter-sheet junction contact resistance ........................................ 10

2.2 Graphene Foam: Alternative to Graphene ………………............................. 11

2.2.1 What is Graphene Foam? ............................................................. 11

2.2.1.1 Fabrication of 3D Graphene Foam........................................ 13

2.2.2 Free Standing Graphene Foam Properties .......................................... 14

2.2.3 Processing of Graphene Foam-Based Polymer Composites .............. 17

2.2.3.1 Dip coating ......................................................................... 18

2.2.3.2 Casting approach .................................................................. 19

2.2.3.3 Electrostatic spray deposition................................................. 19

2.2.3.4 Vacuum infiltration technique.................................................. 20

2.2.4 Properties of Graphene Foam-Based Polymer Composites ................ 21

2.2.4.1 Mechanical Properties ........................................................... 21

2.2.4.2 Electrical properties.............................................................. 24

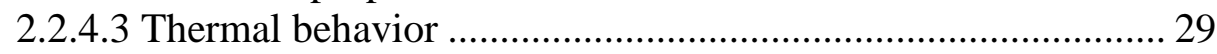

2.2.4.4 Additional Properties of Graphene Foam................................. 32

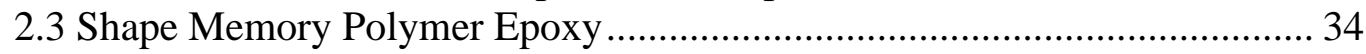

2.3.1 Graphene-Based Shape Memory Epoxy Composites........................... 37

2.3.2 Synthesis of Graphene-Based Shape Memory Epoxy Composites.... 37

2.3.2.1 Graphene Dispersion Technique ............................................ 38

2.3.2.2 Processing of SMP-graphene Composites ............................... 39

2.3.3 Properties of Graphene-Based Shape Memory Epoxy Composites ... 42

2.3.3.1 Shape Recovery Behavior ......................................................... 42

2.3.3.2 Uniaxial and Dynamic Mechanical properties ......................... 44

2.3.4 Potential Applications of Graphene-Based Polymer and SMP

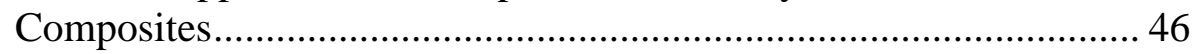

Chapter III: Materials and Methods ...................................................................... 48

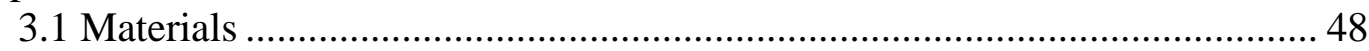

3.1.1 SMP Epoxy Components ………………………………………... 48

3.1.1.1 Diglycidyl ether of bisphenol A ............................................. 48 
3.1.1.2 Neopentyl glycol diglycidyl ether.

3.1.1.3 Poly(propylene glycol)bis(2-aminopropyl) ether ...................... 49

3.1.2 3D Graphene Foam..................................................................... 49

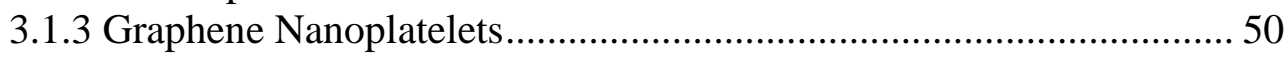

3.2 Experimental Procedure........................................................................... 51

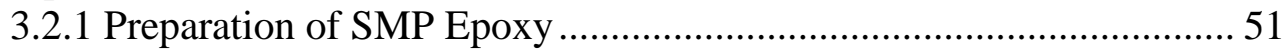

3.2.2 Mold Casting of SMP Epoxy-GrF Composite …………………….... 52

3.2.2.1 Fabrication of Rectangular Shape Samples............................ 52

3.2.2.2 Fabrication of Dog-Bone Shape Samples ................................ 53

3.2.2.3 Fabrication of Electrically Responsive SMP Epoxy-GrF Composites ..................................................................... 54

3.2.3 3D Printing of SMP Epoxy and Graphene-Based SMP Epoxy Composites ................................................................................... 55

3.3 Microstructural Characterization .................................................................. 57

3.3.1 Scanning Electron Microscopy: Powder Morphology and

Microstructure of Composites................................................................ 57

3.3.2 Raman Spectroscopy ................................................................... 57

3.3.2 Fourier Transform Infrared (FTIR) Spectroscopy …………………... 58

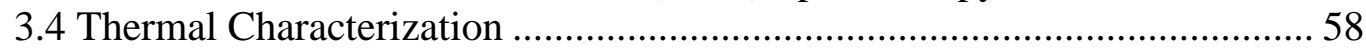

3.4.1 Differential Scanning Calorimetry (DSC) ………………………...... 58

3.4.2 Thermal Conductivity …………………………….......................5 59

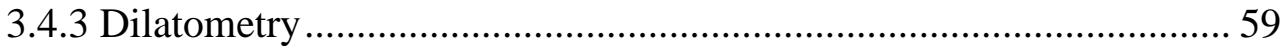

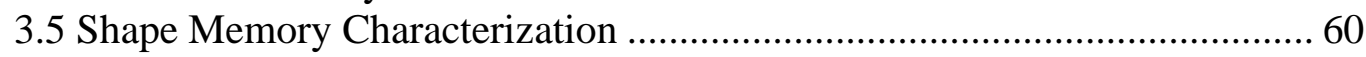

3.5.1 Thermally Stimulated SMP Epoxy-GrF Composite............................. 60

3.5.1.1 Qualitative and Quantitative Evaluation of Shape Recovery of SMP Epoxy-GrF Composite ...................................................... 60

3.5.1.2 Electrically Stimulated SMP Epoxy-GrF Composite................. 61

3.5.3 Thermal Imaging of Electrically Triggered SMP Epoxy-GrF

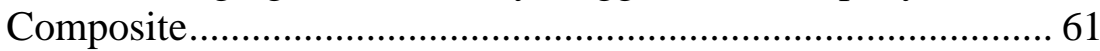

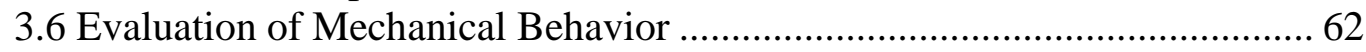

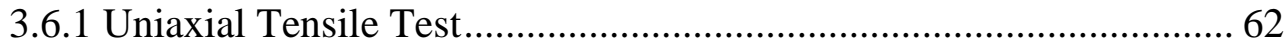

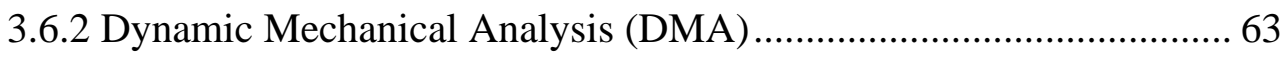

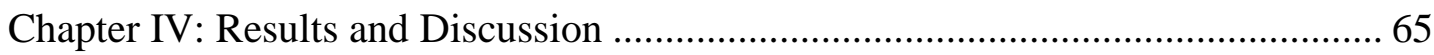

4.1 Morphology of SMP Epoxy-GrF Composites prepared by Mold Casting..... 65

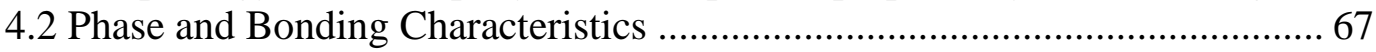

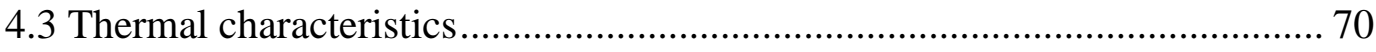

4.4 Thermal actuation of SMP epoxy-GrF composites ....................................... 73

4.4.1 Temporal Gradient Behavior of SMP epoxy-GrF Composite................ 74

4.4.2 Spatial Control of SMP epoxy-GrF Composites via Direct heating .... 79

4.4.3 Effect of switching temperatures on the shape recovery rate of SMP epoxy and SMP epoxy-GrF composite ................................................ 83

4.5 Electrical actuation of epoxy SMP and graph-epoxy SMP composite ..........86 4.6. Proof of concepts of SME in GrF-SMP epoxy shapes and bird composites 92 
Chapter V: Mechanical Behavior of SMP Epoxy-GrF Composite............................. 98

5.1 Uniaxial Tensile Properties ........................................................................ 98

5.1.1 Thermodynamic Response of Mechanical Behavior of SMP epoxy-

$\mathrm{GrF}$

5.2 Dynamic Mechanical Analysis.................................................................. 107

5.2.1 Loss Tangent Behavior of SMP Epoxy Composite Samples under Tensile Deformation ............................................ 108

5.2.2 Storage Modulus and Loss Tangent Behavior of SMP Epoxy-GrF under Bending Deformation ........................................................... 112

5.3 Mechanisms involved in Storage Modulus Behavior...................................... 117

5.4 Mechanisms involved in Loss Tangent Behavior ……………………......... 118

Chapter VI: 3D Printing of SMP Epoxy-Graphene Nanoplatelets Composites by Slurry-Based Extrusion......................................... 125

6.1 SMP Epoxy Precursor for 3D Printing: challenge and solution................... 125

6.1.1 Mixing of SMP liquid and its powder ............................................. 126

6.1.2 Partial curing of SMP liquid at room temperature .......................... 129

6.1.3 Partial curing of SMP epoxy liquid at room temperature (under a nearly cryogenic condition after 3D printing.........................130

6.2 Morphology of 3D printed SMP epoxy-graphene nanoplatelets powder

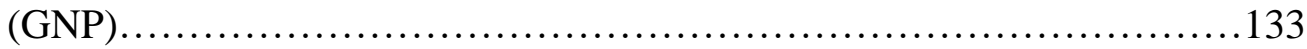

6.3 Phase and Bonding Characteristics ........................................................... 137

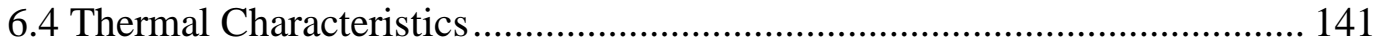

6.5 Shape Memory Behavior of 3D Printed SMP Epoxy-GNP Composite ....... 142

6.5.1 Thermal Actuation......................................................................... 143

6.6 Static and Dynamic Mechanical Properties.................................................... 146

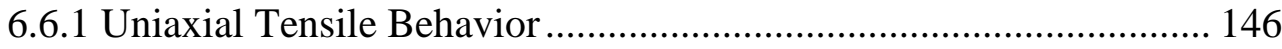

6.6.2 Dynamic Mechanical Properties......................................................... 151

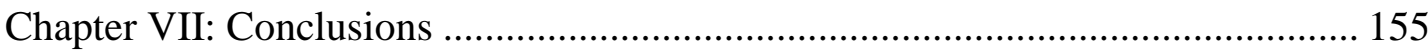

Chapter VIII: Recommendations for the Future Work …………............................. 158

8.1 Optimizing GrF in mold-cast SMP Epoxy Composites ............................... 158

8.2 Surface Treatment of GrF Reinforcement

8.3 Optimizing GNP content in 3D printed SMP Epoxy Composites............... 158

8.4 Control Process Parameters Optimization for 3D printing of SMP Epoxy-

GNP Composite

8.5 Modifications on SMP epoxy precursor and 3D Printing Instrumentation.160

8.6 Fabrication of Kirigami-based SMP Epoxy Composites.................. 160

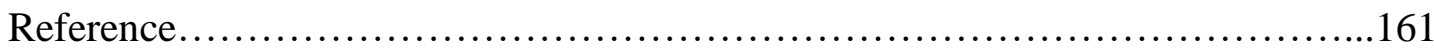

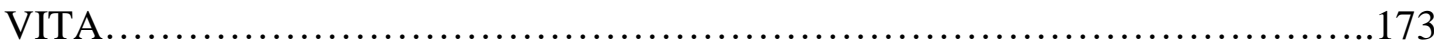




\section{LIST OF FIGURES}

FIGURE

PAGE

Figure 1: Number of publications on GrF properties, ceramic/polymer matrix-GrF composites during 2011 to date....................................12

Figure 2: Classification of 3D Graphene-based frameworks.........................13

Figure 3: Synthesis of Graphene foam: ( $a$ and b) CVD growth of graphene films (Ni-G, b) using a nickel foam as scaffold. (c) as-grown graphene film after coating a thin PMMA supporting layer (Ni-G-PMMA). (d) Graphene foam coated with PMMA (GrF-PMMA) after etching the nickel foam with $\mathrm{FeCl}_{3} / \mathrm{HCl}$ solution

(e) Free-standing GrF after PMMA dissolution with acetone.

Figure 4: In-situ, multiscale, and dynamic analysis for determination of properties and failure mechanisms of free-standing graphene foam....................16

Figure 5: Meso-structure evolution during tensile deformation. (a) Beginning state of GrF in uniaxial tension (b) End state of GrF uniaxial tensile deformation; Yellow color represents local graphene flakes. (c) four sequential thermodynamic states of mesostructure during tensile deformation.

Figure 6: Schematic showing dip-coating process involving polymer-graphene foam coating process ................................................ 18

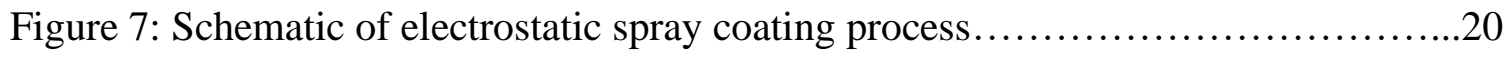

Figure 8: Schematics showing failure mechanisms and influence of processing on the behavior of graphene foam-based polymer composites fabricated by coating

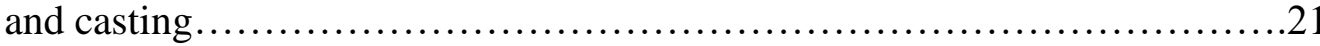

Figure 9: Stress-strain graph of Graphene foam. i) black line - 70\% loading strain; ii) red line $-60 \%$ loading strain; iii) blue line $-40 \%$ loading strain; iv) purple line $-20 \%$ loading strain.

Figure 10: Stress-strain graph of GrF/PDMS. i) black line - 70\% loading strain; ii) red line $-60 \%$ loading strain; iii) blue line $-40 \%$ loading strain; iv) purple line $20 \%$ loading strain.

Figure 11: (a). Electrical conductivities of GrF/PDMS (in-plane) vs Graphene wt.\%. (b) electrical conductivities of GrF/PDMS (through-plane) vs Graphene wt.\%. 
Figure 12: Electrical conductivities of GrF/PDMS \& GrF/Epoxy vs GrF wt.\%

Figure 13: Scheme of thermal and electron transport in composites reinforced with 1D,

$2 \mathrm{D}$ and $3 \mathrm{D} \mathrm{GrF}$.

Figure 14: Graph of thermal conductivity vs temperature of PI and 0.35 wt.\% GrF/PI at $150^{\circ} \mathrm{C}$, and PDMS and 0.7 wt. $\%$ GrF/PDMS at $85^{\circ} \mathrm{C}$

Figure 15: Graph of thermal conductivity vs filler content of Polyamide/GrF, Multigraphene flakes/PDMS, Multigraphene flakes/GrF/PDMS.

Figure 16: Chemical Structures of SMP epoxy components (a) Diglycidyl ether bisphenol A (DGEBA); (b) Neopentyl glycol diglycidyl ether (NGDE); (c) Jeffamine D-230 (n 2.5); (d) decylamine ..................................35

Figure 17: Number of publications on SMP reinforced with carbon-based fillers during 2015-date (Source: www.webofknowledge.com).

Figure 18: Preparation procedures of RGO/WEP/RGO sandwich structure composite film

Figure 19: Schematic of the fabrication of E-G composites. (a) Preparation of GO aqueous suspension with THF, then freezing by bidirectional freeze-casting (b) The lamellar GO scaffold was obtained after freeze drying (c) Subsequently, heating was applied to reduce the GO scaffold, forming an rGO scaffold (d). Finally, the E-G composite was produced by infiltrating with shape memory EP and (e) curing.

Figure 20: Surface images of the as scratched and recovered surfaces. The optical images in the left two columns represent samples after scratch testing. The optical images in the third column are the recovered samples after heating.

Figure 21: (a) Stress-strain curves of GO-toughened epoxy resin composites (b) DMA curves of storage of GO-toughened epoxy resin composites....

Figure 22: (a) SEM micrograph of as-received macro-porous graphene foam made by chemical vapor deposition (CVD) (b) SEM Image of as-received graphene nanoplatelets (xGNP-M-5) were obtained from XG Sciences..............51

Figure 23: Schematic of a Mold Cast Thermal Responsive SMP-GrF Composite........53

Figure 24: Reverse impression dog-bone mold fixture $\ldots \ldots \ldots \ldots \ldots \ldots \ldots \ldots \ldots \ldots \ldots . .54$ 
Figure 25: Schematic of SMP epoxy-GrF mold cast Composite......................54

Figure 26: Schematic of a fabricated Electrically Responsive SMP-GrF Composite......55

Figure 27: Image showing mechanical MTI testing stage used for tensile testing...........62

Figure 28: Fractured surface of SMP epoxy-GrF composites revealing graphene wrinkled surface and graphene flake like structure at (a) low and (b) high magnifications....

Figure 29: Raman spectrum of GrF and SMP epoxy-GrF Composite revealing peaks characteristics of $\mathrm{GrF}$ and peak shifts in the composite.

Figure 30: FTIR spectra of GrF, SMP epoxy and SMP epoxy-GrF composite confirming possibililty in interfacial interactions between functional groups on SMP epoxy and GrF.

Figure 31: Differential Scanning Calorimetry of SMP epoxy and SMP epoxy-0.13 wt.\% GrF composite displaying their glass transition behaviors.

Figure 32: (a) Illustration of the Dual Component Mechanism (DCM) in SMP epoxy

(b) Dual Component mechanisms of enhanced performance for 2D

Graphene addition, (c) the mechanisms of improved performance for 3D graphene foam-based additions.

Figure 33: Shape recovery images of (a) SMP epoxy and

(b) SMP epoxy-0.75 wt.\% GrF composite triggered by direct heating from hot plate.

Figure 34: Shape recovery angle as a function of time for epoxy SMP and graphene foam-epoxy SMP composite subjected under direct heating from hot plate.

Figure 35: Shape recovery images of thermally actuated (heated air)

(a) SMP epoxy and (b) 0.75 wt.\%GrF-epoxy SMP composite stimulated by heated air from heat gun.

Figure 36: Shape recovery angle as a function of time for epoxy SMP and graphene foam-epoxy SMP composite heated with hot air .78

Figure 37. Thermal Flir imaging of SMP-GrF samples. a) stimulated by direct heating (hot plate); thermal gradient of b) direct heating of SMP; c) direct heating of SMP-GrF composite showing special control via GrF filler d) Thermal gradient of thermally stimulated SMP Epoxy and SMP Epoxy-GrF Composite as a function time, at $70^{\circ} \mathrm{C}$ 
Figure 38: Thermal conductivity behavior of SMP Epoxy and SMP epoxy-0.5 wt.\% $\mathrm{GrF}$ at 25 and $70^{\circ} \mathrm{C}$

Figure 39: Shape recovery rate of SMP epoxy and SMP epoxy-0.5 wt.\% GrF as a function of switching temperatures

Figure 40: Glass transition behavior of $0.5 \mathrm{wt} \%$ graphene foam-epoxy SMP as a function of T-M cycles.

Figure 41. Temporal gradients of fold-deploy tests of SMP epoxy-GrF composites.

a) stimulated by direct heating (hot plate); b) triggered by indirect or

b) electrical heating at $0.4 \mathrm{~A}$.

Figure 42. Thermal Flir images of SMP epoxy actuated by indirect (electrical) heating at a) $0.4 \mathrm{~A}$ and SMP epoxy-GrF samples at b) $0.1 \mathrm{~A} \mathrm{c}) 0.2 \mathrm{~A} \mathrm{~d}$ ) $0.3 \mathrm{~A} \mathrm{e)} 0.4$ A f) thermal gradient of fold-deploy tests of electrical heating of SMP composite showing spatial control via GrF filler...

Figure 43: a) Images of SMP epoxy fabricated to S-shape, deformed to thin rectangular shape and recovered back to $\mathrm{S}$-shape under the influence external heat stimulus; b) Images of SMP epoxy designed to L-shape. deformed into rectangular shape and recovered back to L-shape when subjected to heat trigger; c) Images of graphene foam- SMP epoxy made into inverted U-shape deformed into irregular rectangular shape and recovered back to inverted U-shape when stimulated by heat.

Figure 44: a) Images of SMP epoxy fabricated into accordion-like shape, stretched out into temporary shape and restored to its original shape under the influence external heat from hot water; b) Images of graphene- SMP epoxy made into accordion-like shape, deformed into temporary shape and recovered to its original shape when triggered by thermal actuation; c) Images of graphene-foam- SMP epoxy composite made into accordion-like shape, deformed into temporary shape and recovered to its original shape when stimulated under heat..........................................94

Figure 45: a) Images of SMP epoxy fabricated into accordion-like shape, stretched out into temporary shape and restored to its original shape under the influence hot air; b) Images of graphene foam- SMP epoxy made into accordion-like shape, deformed into temporary shape and recovered to its original shape when triggered by hot air.... 
Figure 46: (a) Images of deformed (temporary shape) wings of epoxy SMP bird and b) graphene foam-epoxy SMP bird at $25^{\circ} C$; c) Images of recovery of the deformed wings of epoxy SMP bird and d) graphene foam-epoxy SMP bird e) Image of cracked-open wing of biomimetic graphene foam-epoxy SMP bird after $15^{\text {th }} \mathrm{T}-\mathrm{M}$ cyclic deformation.; f) Images of crack-closed wing of graphene foam-epoxy SMP bird.

Figure 47: (a) Uniaxial tensile stress-strain curves for SMP epoxy and mold cast composite of $0.5 \mathrm{wt} \% \mathrm{GrF}$ content (b) Comparison of Young modulus (elastic modulus) of SMP epoxy and composite of $0.5 \mathrm{wt} \% \%$ GrF content ....99

Figure 48: SEM images of fracture surface of SMP epoxy-GrF composite. (a) GrF branch-node anatomy retaining its integrity in SMP epoxy after curing (b) shrinkage of SMP epoxy leading to interfacial spacing between GrF and SMP epoxy (c) distinct surface features of GrF and SMP epoxy showing higher magnification of the interfacial spacing (d) close view of good interface regions between $\mathrm{GrF}$ and SMP epoxy

Figure 49: Schematic showing a) Stretching of entangled polymers chains with high entropy state; b) stretched, aligned polymer chains with low entropy state.

Figure 50: Schematic portraying highly randomly coiled position of SMP epoxy after mixing the three epoxy resins component without curing and chain deformation.

Figure 51: Schematic portraying (a) highly stretched SMP epoxy chain and

(b) SMP epoxy-GrF samples after curing and deformation 106

Figure 52: Plot of loss tangent versus of SMP Epoxy and SMP Epoxy-0.5 wt.\% GrF a) as a function of number of cycles; as a function temperature at b) $30 \mathrm{~Hz}$; ) $100 \mathrm{~Hz}$ .110

Figure 53: Plot of storage modulus versus temperature of SMP epoxy and SMP epoxy-0.5 wt.\% GrF at amplitude of (a) $50 \mu \mathrm{m}$ (b) $100 \mu \mathrm{m}$ and (c) $200 \mu \mathrm{m}$

Figure 54: Plot of loss tangent versus temperature of SMP epoxy and SMP epoxy-0.5 wt.\% GrF at amplitude of (a) $50 \mu \mathrm{m}$ (b) $100 \mu \mathrm{m}$ and (c) $200 \mu \mathrm{m}$; (d) Plot of distance points against inversion temperature of SMP Epoxy and SMP Epoxy-0.5 wt.\% GrF

Figure 55: (a) van deer Waals interactions between graphene sheets, and (b) bending of $\mathrm{GrF}$ branch during applied load from 3-point bending test

Figure 56: Plot of Coefficient of thermal expansion vs temperature of SMP epoxy and SMP-0.5 wt.\% GrF Composite.

Figure 57: Plot of Coefficient of thermal expansion vs temperature of GrF (a) 1 cell; 
(b) 4 cells and (c) Bulk cells.

Figure 58: (a) Print head syringe for low viscosity polymers.

125

Figure 59: (a) SEM micrograph showing varying sizes of SMP epoxy powder processed by ball milling technique (b) particle size distribution of SMP epoxy powder...

Figure 60: Image showing 3D printed dog-bone shape sample using mixture of SMP epoxy liquid and powder.

Figure 61: Image of 3D printed dog-bone shape sample using partial cured SMP epoxy precursor. (a) immediately after printing (retaining the dog-bone shape geometry); (b) 30 minutes after printing (revealing smudging of SMP epoxy and not retaining the shape geometry)....

Figure 62: Images of 3D printed dog-bone shape sample placed under nearly cryogenic condition. a) SMP epoxy b) SMP epoxy-0.1 wt.\% GNP

Figure 63: SEM micrographs of cross-section of 3D printed dog-bone shape specimen from SMP epoxy top view surface (a) at lower magnification (150×); (b) at higher magnification $(1500 \times$ and $5000 \times$ [inset]) and side view surface (c) at lower magnification $(100 \times)$ and (d) at higher magnification $(750 \times)$

Figure 64: SEM micrographs of cross-section of 3D printed dog-bone shape specimen from SMP epoxy-0.1 wt.\% GNP (a) at lower magnification (150×); (b) at higher magnification (1500× and 5000× [inset]) and SMP epoxy-0.1 wt.\% GNP (c) revealing rough surfaces and (d) interface between graphene and SMP epoxy.

Figure 65: Raman spectrum of (a) GNP powder []; Raman spectra of SMP epoxy, SMP epoxy-0.1 wt.\% GNP and SMP epoxy-0.25 wt.\% GNP at (b) wavenumber $1000-1800 \mathrm{~cm}^{-1}$; (c) at wavenumber $2000-4000 \mathrm{~cm}^{-1} \ldots . .138$

Figure 66: FTIR spectra of SMP epoxy and SMP epoxy-0.1 wt.\% GNP composite confirming possibility of interfacial interactions between functionalities on SMP epoxy and graphene.

Figure 67: Differential Scanning Calorimetry (DSC) curves of 3D printed SMP epoxy and SMP epoxy-0.1 wt.\% GNP Composite.

Figure 68: Shape recovery images of pre-deformed samples actuated by direct heat from hot plate (a) SMP epoxy and (b) SMP epoxy-0.1 wt.\% GNP composite.

Figure 69: Shape recovery images of pre-deformed SMP epoxy and SMP epoxy-0.1 wt.\% GNP composite samples actuated by direct heat from hot plate heat source. 
Figure 70: Images of 3D printed dog-bone shape samples fabricated by using syringe extruder (a) room cured SMP epoxy (b) room cured SMP epoxy-GNP.....147

Figure 71: (a) Uniaxial tensile stress-strain curves for mold-cast SMP epoxy and SMP epoxy composite of $0.1 \mathrm{wt}$ \% GNP content (b) Comparison of Young modulus of SMP epoxy and composite of $0.1 \mathrm{wt} . \%$ GNP content...148

Figure 72: SEM micrographs of cross-section of 3D printed SMP epoxy revealing smooth surface feature.

Figure 73: SEM fractographs of SMP epoxy-GNP composite. (a) image taken from perpendicular planes to the layer deposition axis, with $2200 \times$ [inset] (b) image taken from parallel plane to the layer deposition axis, with $750 \times$

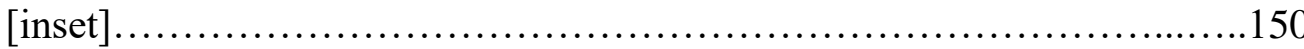

Figure 74: SEM images of the fracture surface showing crack-bridging mechanism in SMP epoxy-GNP composite. (a) low magnification of $1500 \mathrm{X}$ and (b) high magnification of 5000X............................................... 151

Figure 75: Plot of (a) storage modulus and (b) tan delta of SMP epoxy and SMP epoxy- $0.1 \mathrm{wt} . \%$ GNP composite at amplitude load of $5 \mu \mathrm{m}$ 


\section{Chapter I: Introduction}

The aim of this study is to fabricate GrF-reinforced shape memory epoxy composites. The motivation is to develop a smart polymeric composite that addresses the downsides of shape memory epoxy, such as low thermal conduction, poor electrical conduction, low recovery stress, by integrating GrF reinforcement in shape memory epoxy. A thorough analysis of shape memory effect, thermal and mechanical properties of the newly developed composites are shown to attest to its superior performance over shape memory epoxy.

\subsection{Shape Memory Polymers and Their Applications - Advantages, Drawbacks and Solution}

The growing interest and demand on innovative materials have led to continuous development of smart materials within the past two decades [1]. Smart materials are responsive materials that can change its physical properties when activated by a specific external stimulus. Appreciable progress in the actuation of these materials has been attained in this research field [2]. The progress has enabled other stimuli sources (electricity, light, magnetic field), other than heat, to trigger response from smart materials [3],[4]. The Technological importance of these materials in the past decade became noticeable due to their wide range of applications. The broad utilization of smart materials ranges from conventional applications in electronics [5], textiles [6] and packaging [7] to elegant applications presently developed in biomedicine [8], automotive and aerospace [9]. Smart

materials are based on different material systems. These material systems are classified as magnetostrictive, chromogenic and piezoelectric, hydrogels, electroactive polymers, shape memory alloys and shape memory polymers (SMP) systems [10], [11]. 
SMP is one of the most extensively used smart materials, engineered from conventional polymers to stimulus-responsive systems. As emerging class of advanced polymers, they possess the ability to change their shape when appropriately stimulated. Once fabricated into their original, permanent shape, SMP can be deformed and retained as a temporary shape. The temporary shape is fixed till it is induced by an external stimulus, resulting to recovery of the original shape. This phenomenon is termed shape memory effect (SME), meaning that SMP can be deformed into temporary shape (shape fixity) and triggered to memorize or remember its shape (shape recovery). SME in SMP can be combined successfully with other functional properties such as thermal and electrical conductivity, biocompatibility and biodegradability, thus resulting in multifunctional SMP [12]. Recently, SMP epoxy has received more attention primarily due to its facile fabrication, low cost and light weight [13]-[15]. SMP epoxy possess additional advantages such as its high recoverable strain, robust structural versatility, synthetic flexibility, environmental durability and industrial viability [16], [17]. These advantages have made SMP epoxy to be highly considered as a matrix component for composite system ahead of its shape memory alloy counterpart, in some specific applications.

Nevertheless, SMP have some downsides which have limited its ability and constrained its application boundaries from being expanded into new area of possibilities. The limitations associated with SMP mainly includes poor thermal conductivity $(<0.3$ $\mathrm{W} / \mathrm{mk})[18]$, low storage modulus (1.4 - 2.5 GPa) [19], [20], and low recovery stress (1 $3 \mathrm{GPa}$ [21]. It construes that poor intrinsic stiffness of SMP epoxy accounts for the low recovery stress generated during shape recovery. Poor heat transfer leads to slow thermal 
diffusion through the SMP epoxy. This translates to slower recovery rate and longer recovery period for the SMP epoxy to attain its permanent shape. A possible approach to eliminate the drawbacks is by adding a reinforcement material. Addition of mere low content of the reinforcement can pose great influence on the structure-property characteristics of the SMP epoxy matrix [22]. It is therefore pivotal that such reinforcement possesses remarkable capabilities. These capabilities can be used to imbue certain desirable attributes, such as enhanced shape recovery, stiffness, and thermal and electrical conductivity, into SMP epoxy.

\subsection{Graphene Reinforcement - Its Capabilities to Shape Memory Polymers}

Graphene, a basic building block of carbon-based nanomaterial, is a single layer of $\mathrm{sp}^{2}$ bonded carbon atoms organized in a hexagonal, two-dimensional honeycomb lattice [23]. Atomic configuration of graphene has enabled it to exhibit remarkable elastic modulus (1 TPa), tensile strength (130 GPa) [24], thermal conductivity (5000 W/mK) [25], temperature stability of up to $1500^{\circ} \mathrm{C}$, and electrical conductivity $(72 \mathrm{~S} / \mathrm{cm})[26]$. All these promising behaviors have designated graphene to be highly preferred as a versatile filler, reinforcing the three main material matrices (ceramic, metal and polymers). In a conventional polymers system, graphene reinforcement has been able to augment the elastic modulus, fracture toughness and tensile strength of the polymers [27]. In addition, it has demonstrated great ability to improve glass transition and thermal conduction coupled with a boost in electron mobility within the polymers. 


\subsubsection{Challenges Associated with Graphene Reinforcement}

Graphene's exceptional properties have great potential to boost the material properties of the polymers. The transformation would thereby create a new breed of highly advanced polymeric composites. However graphene addition in polymers has not lived up to desired expectations in improvement in the properties [28]. The inability to fully harness its properties can be traced to the intrinsic nature of graphene. Graphene has s high surface energy $\left(78.9 \pm 3.1 \mathrm{~mJ} / \mathrm{m}^{2}\right) \mathrm{s}[29]$ resulting in the non-homogeneous dispersion of graphene sheets [30], weak interfacial interaction between polymers [31], and high intersheet junction contact resistance [32]. Due to the presence of weak intermolecular forces, graphene sheets reaggregate when dispersed in polymer matrices. This makes its uniform dispersion burdensome and poses a serious challenge for processing of polymer composites. Besides the processing challenge, graphene agglomeration can serve as stress concentrators, which can lead to failure of the resultant composites.

Graphene reinforcement in polymers tends to have discontinuity zones, thereby affecting the performance of polymer composites. The discontinuity zones are formed due to make-up of the graphene structure. From the atomic and molecular perspective, graphene planes consist of carbon atoms. These atoms are held together by aromatic bonds. The bonds account for graphene inertness and chemical stability [31]. Graphene therefore has no other alternative than to relate with polymers through weak intermolecular forces. Thus, the interfacial bond strength formed between the reinforcement and matrix can act as a weak link in the composites. In addition, surface chemistry between polymer molecules and between graphene sheets creates low surface energy between the two components. It construes that covalent bond in the polymer molecules and the weak 
intermolecular forces between graphene layers account for the low surface energy. Graphene reinforcement thus experiences poor wettability by polymers due to the low surface energy [33].

\subsection{D Graphene Foam: Suitable reinforcement to shape memory polymer epoxy}

The challenges associated with graphene require an efficient, suitable reinforcement to SMP epoxy. The suitable reinforcement should possess properties like graphene as well as ability to resolve agglomeration issues in graphene. Graphene foam ( $\mathrm{GrF})$ has the capacity to act as an alternative. GrF is a carbon-based nanomaterial, consisting of twodimensional graphene sheets arranged into a three-dimensional architecture. It is an interconnected 3D continuous framework of nodes and branches. It displays ultralow density $\left(<0.005 \mathrm{~g} / \mathrm{cm}^{3}\right)$, high surface energy and large specific surface area $\left(850 \mathrm{~m}^{2} / \mathrm{g}\right)$ elastic modulus (69.9 GPa) and specific tensile strength $\left(1.2 \mathrm{~Pa} \cdot \mathrm{m}^{3} / \mathrm{Kg}\right)$ [34]. These interesting properties position $\mathrm{GrF}$ as a potential reinforcement for SMP epoxy.

The 3D GrF architecture allows for flexibility and elasticity, enabling it to withstand multiscale mechanical deformation and exhibit great damping characteristics. The branchnode morphology of GrF creates electron and thermal flow paths that facilitate its high electrical and thermal conductive properties. GrF ability to exhibit these attributes makes it a very attractive multifunctional reinforcement in polymer/ceramic/metal matrices [23]. Particularly, these multifunctional properties of GrF can be fit for advanced polymers, such as in shape memory epoxy, for several applications. Such applications include aerospace field (flexibility of aircraft wings), electronic purposes (nano and micro-electromechanical systems) and biomedical area (wearable sensor and nerve tissue generation). 


\subsection{Objectives of Current Research}

The overall aim of this research is to enhance the shape memory response of graphene and GrF-reinforced SMP epoxy composites for structural applications. The overall objective could be accomplished through the following specific objectives:

- Reinforce SMP epoxy with GrF and GNP using mold casting approach and syringebased extrusion 3D printing, respectively

- Understand the free flow infiltration of SMP epoxy in GrF and role of GrF uniform distribution in SMP epoxy matrix

- Influence of GrF-SMP epoxy interface behavior on the overall performance of the composite.

- Effect of GrF on glass transition temperature, thermal expansivity characteristics and conductivity of SMP epoxy.

- Role of GrF and GNP reinforcement in the shape recovery of thermally responsive SMP epoxy composite

- Influence of GrF reinforcement in the shape recovery of electrically stimulated SMP epoxy composite

- Examine mechanical properties in terms of elastic modulus, tensile strength, storage modulus, and loss tangent of GrF and GNP-based SMP epoxy composites.

The research work conducted in this study has been organized into chapters, sections, and subsections of this dissertation. Chapter II presents an exhaustive literature review on this research topic. It also features the growing interest of this topic in the scientific community. Chapter III conveys a detailed description of materials and methodology 
employed in the preparation, characterization and evaluation of GrF-reinforced SMP epoxy composites. In-depth discussion and scientific analysis of the results in line with the aim of this research are provided in chapters IV, V and VI. Chapter VII gives the main points of the study, summarizing key outcomes. Future research work and recommendations about graphene based SMP composites are highlighted in chapter VIII. 


\section{Chapter II: LITERATURE REVIEW}

\subsection{Graphene-Based Polymer Composites}

The significant attention that carbon-based polymer composites has received over the past two decades is largely due to its light weight, high strength-to-weight ratio, ease of processing and usefulness in many engineering applications [35]-[37]. Intrinsic properties of the carbon nanofillers, such as high aspect ratio (1000-1272) [38], [39], specific surface area $\left(150-2600 \mathrm{~m}^{2} / \mathrm{g}\right)[40]-[42]$, etc., have considerably accounted for the improvements in the behavior of the composites [43], [44]. Thus, it has facilitated tremendous progress in the study of carbon-based fillers, including graphene, in polymer composites [45]-[47]. Graphene is a two-dimensional monolayer of $\mathrm{sp}^{2}$ bonded carbon atoms organized in a hexagonal lattice. Graphene reinforcement in different polymer matrices has increasingly gained attraction after its discovery in 2004 [48]. The attraction is due to its remarkable mechanical, thermal and electrical properties. Nevertheless, its fascinating properties have not been fully harnessed in polymer matrices. The constraints associated with the completely utilizing graphene properties by different polymers can be attributed to inherent behavior of graphene. Main challenges in graphene reinforced polymer composites (GRPC) include: (i) weak interfacial interaction between graphene and polymer matrices, (ii) non-uniform distribution of graphene sheets within the polymer, and (iii) high intersheet junction contact resistance.

\subsubsection{Interfacial interactions between graphene filler and polymers}

Polymers reinforced with graphene have different discontinuity zones or interfaces which significantly influence the overall behavior of polymer composites. Properties of the 
polymer matrix and graphene filler are two key determining factors for the compatibility and adhesion of the interfaces formed in GRPC. Moreover, graphene structural make-up and behavior at the interface of the composite influence the interactions between polymer/graphene interface [49]. From the atomic and molecular point of view, the carboncarbon bonds within the graphene planes are aromatic. The aromaticity has accounted for graphene chemical stability and inertness [31]. It consequently left graphene with the only option of interacting with the polymer chains through weak intermolecular forces. This results in low interfacial bond strength between the graphene and polymers [50]. The wrinkled or pristine surface of graphene can create presence or absence of physical interlocking positions respectively with polymers, which has affected the interfacial properties. While pristine graphene indicates that integrity of its overall properties is untouched, the absence of physical interlocking of graphene with the polymer chains does not allow very good interface formation [51]. Property trade-off is thus required to attain excellent interfacial interaction. Wetting behavior of graphene, which stems from graphene's surface chemistry, has also affected the interface behavior in GRPC [52]. Due to the covalent bonds between polymer molecules and weak intermolecular forces between graphene layers, low surface energy dominates between the two components. This creates poor wettability of graphene by the polymer.

\subsubsection{Dispersion of graphene within the polymers}

To achieve isotropic properties in GRPC, dispersion of graphene within a polymer matrix is an essential factor to be considered. The dispersive behavior of graphene remains a germane concern to achieve suitable reinforcement in GRPC. High specific surface area $\left(2630 \mathrm{~m}^{2} / \mathrm{g}\right)$ and physical attraction between graphene sheets owing to its high surface 
energy have created leverage for graphene sheets to agglomerate. Its high aspect ratio makes the sheets to be susceptible to restacking, thereby preventing the homogenous distribution within the entire polymer matrix [53]. Also, the presence of weak physical forces such as intermolecular interactions (very significant at nanoscale) have considerably accounted for graphene sheets reaggregation [54].

\subsubsection{High inter-sheet junction contact resistance}

Interfacial contacts, such as plane-to-plane, plane-to-edge, and edge-to-edge, can occur among small-sized graphene sheets incorporated within a polymer matrix [55]. These contacts are, however, not completely achieved due to polymer chains in between or covering the low content of graphene filler. This hinders the percolation limit from being reached and results in poor electrical and thermal conductivities. The presence of functional groups between the functionalized graphene platelets and polymers can initiate high intersheet junction contact resistance among the graphene platelets [32]. Long range of connected graphene sheets is thus inhibited. Percolation of electrons and phonons between graphene sheets is also restrained. This culminates in overall decrease of electrical and thermal conduction in the resultant polymer matrix composites (PMC).

Different approaches have been adopted to resolve the challenges precluding the full expression of graphene properties within polymers. Such approaches can be mainly grouped into two: (i) assembling and (ii) functionalization techniques. Assembly approach is primarily used to prevent graphene sheet agglomeration. Different types of graphene sheet assemblies are vertically oriented graphene sheets and deformed graphene sheets

which includes curved graphene, folded graphene, crumpled graphene and spaced graphene nanosheets [56]. Functionalization of graphene sheets is employed to improve 
interactions between the polymers and graphene. This can be achieved by properly functionalizing its edges and surface with chemical groups which have a strong affinity for side chain polymers [57], [58]. While the approaches have facilitated improvements in GRPCs, more of graphene remarkable properties can still be explored using the 3D graphene-based architecture, such as graphene foam.

\subsection{Graphene Foam: Alternative to Graphene}

Graphene foam is considered as a great substitute to graphene for reinforcing polymers for structural and multi-functional applications. The design of graphene to a three-dimensional framework has great capabilities to minimize constraints associated graphene when integrated in polymers. Detailed description about GrF nanomaterial, its synthesis and its influence as reinforcing agent in property enhancement of polymer composites are presented in the following subsections.

\subsubsection{What is Graphene Foam?}

Graphene foam $(\mathrm{GrF})$, one of the types of 3D graphene architectures, is a threedimensional (3D) nano-carbon material. It is a macro-porous, continuous 3D network of graphene sheets. GrF framework has pore size of $580 \mu \mathrm{m}$. The graphene sheets network consists of hollow branches and node junctions. The hollow branch has diameter of $50 \mu \mathrm{m}$. GrF branch-node anatomy enables seamless pathways of phonons, electrons and stress transfer. Its architecture thus boosts graphene foam capacity to serve as a multi-functional reinforcing agent. GrF macro-porous framework enables it to have an ultra-low density of $4 \mathrm{mg} / \mathrm{cm}^{3}$. This makes $\mathrm{GrF}$ a desirable alternative for light weight reinforcement in conventional and smart polymer composites. GrF has large specific surface area $\left(850 \mathrm{~m}^{2} / \mathrm{g}\right)$ and can exhibit remarkable electrical conductivity $(125 \mathrm{~S} / \mathrm{cm})$ [48], thermal conductivity 
$\left(0.26-1.7 \mathrm{~W} \mathrm{~m}^{-1} \mathrm{~K}^{-1}\right)$ [49], elastic modulus and other mechanical properties [50]. Figure 1 conveys the increasing trend in the number of publications on GrF and GrF-reinforced composites for the past 9 years. GrF-polymer matrix composites (PMC) has a obvious increase in its number of publications compared to GrF-based metal and ceramic composites. The noticeable attention towards GrF-PMC can be primarily attributed to the simple synthesis of polymer composites. It does not demand high temperature and pressure conditions for its fabrication, which is needed in the processing of metal and ceramic composites.

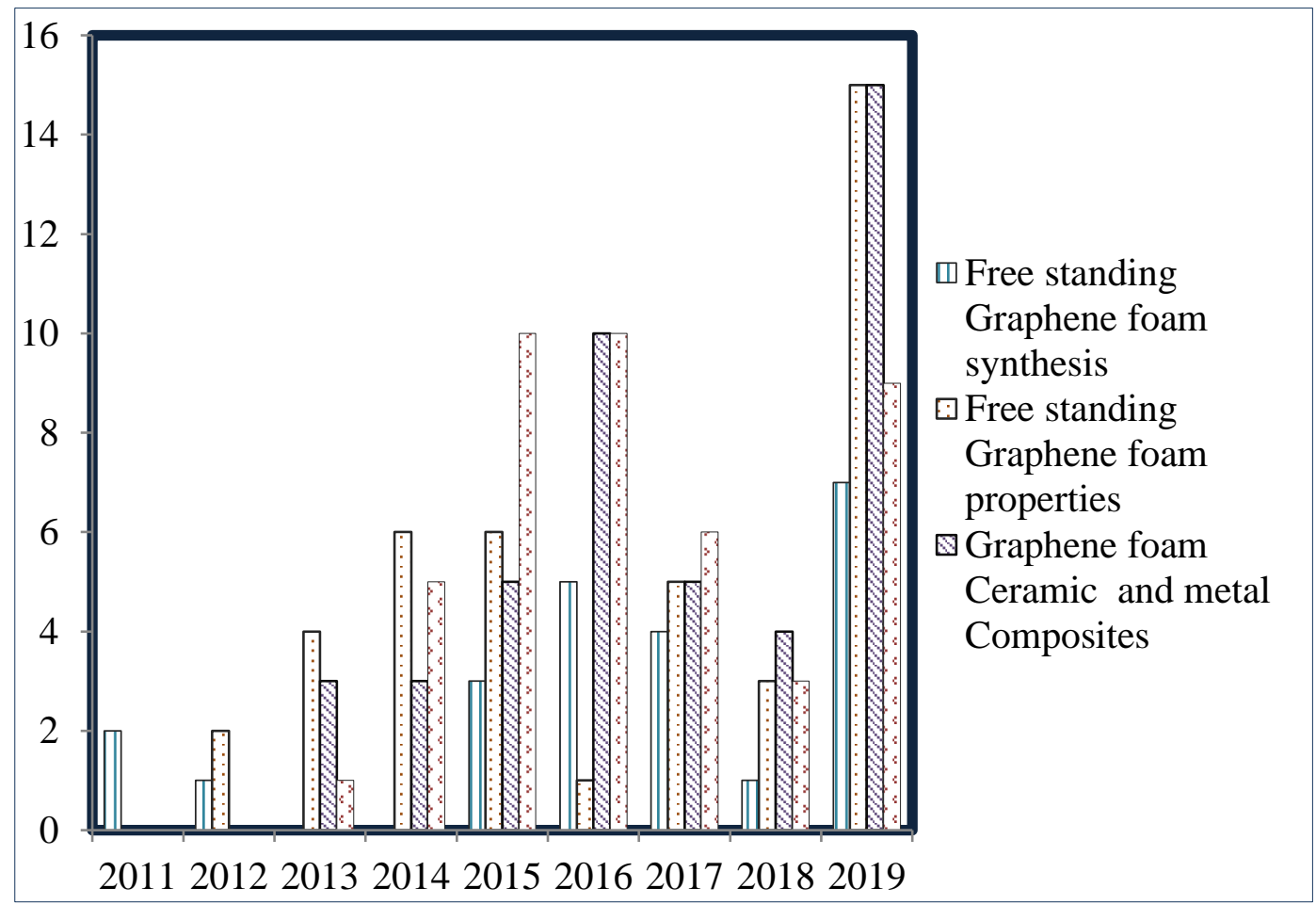

Figure 1: Number of publications on GrF properties, ceramic/polymer matrix-GrF composites during 2011 to date [23]. 


\subsubsection{Fabrication of 3D Graphene Foam}

3D graphene-based frameworks can be classified into aerogels/hydrogels and foambased structures as shown in figure 2. The approaches used to synthesis hydrogels and aerogels include freeze-drying [51], non-template approach [52]-[54], electrochemical reduction [55], sugar blowing approach [56], sol-gel reaction [57] and template assembly of graphene oxide [58]-[60], assembly of graphene oxide (GO) [61]-[63] (including selfassembly of graphene oxide), cross-linking agent [64], 3D printing [65], commercial graphite paper technique [66] and chemical vapour deposition (CVD).

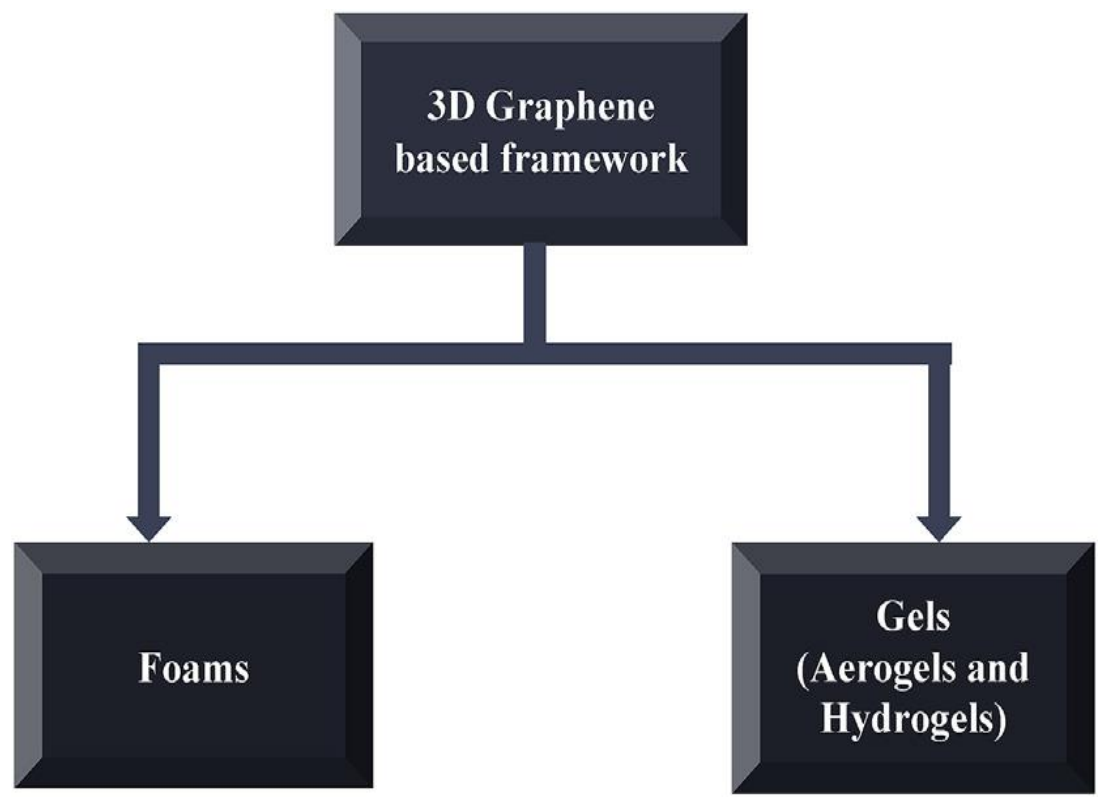

Figure 2: Classification of 3D Graphene-based frameworks [23].

Typical fabrication of 3D GrF is based on the template-directed CVD technique [67]. The technique begins with the selection of nickel foam (a porous, interconnected 3D scaffold of nickel) as a pattern for the $\mathrm{GrF}$ growth. Then decomposition of $\mathrm{CH}_{4}$ at $1000^{\circ} \mathrm{C}$ under ambient pressure provides and releases carbon source onto the nickel foam. Next step is the precipitation of graphene films, where the wrinkle formation is observed [68], [69]. 
Then, an attenuated layer of poly (methyl methacrylate) on the graphene film surface is deposited, precluding the disintegration of graphene network during nickel removal. Subsequently, $\mathrm{HCl}$ (or $\mathrm{FeCl}_{3}$ ) was subsequently used to etch away the nickel foam, as shown in figure 3 Acetone was finally employed to cautiously remove PMMA, resulting in a monolith interconnected 3D graphene network. Graphene foam continuous network and high porosity $(99.7 \%)$ that are created facilitates uniform dispersion in composite matrices.
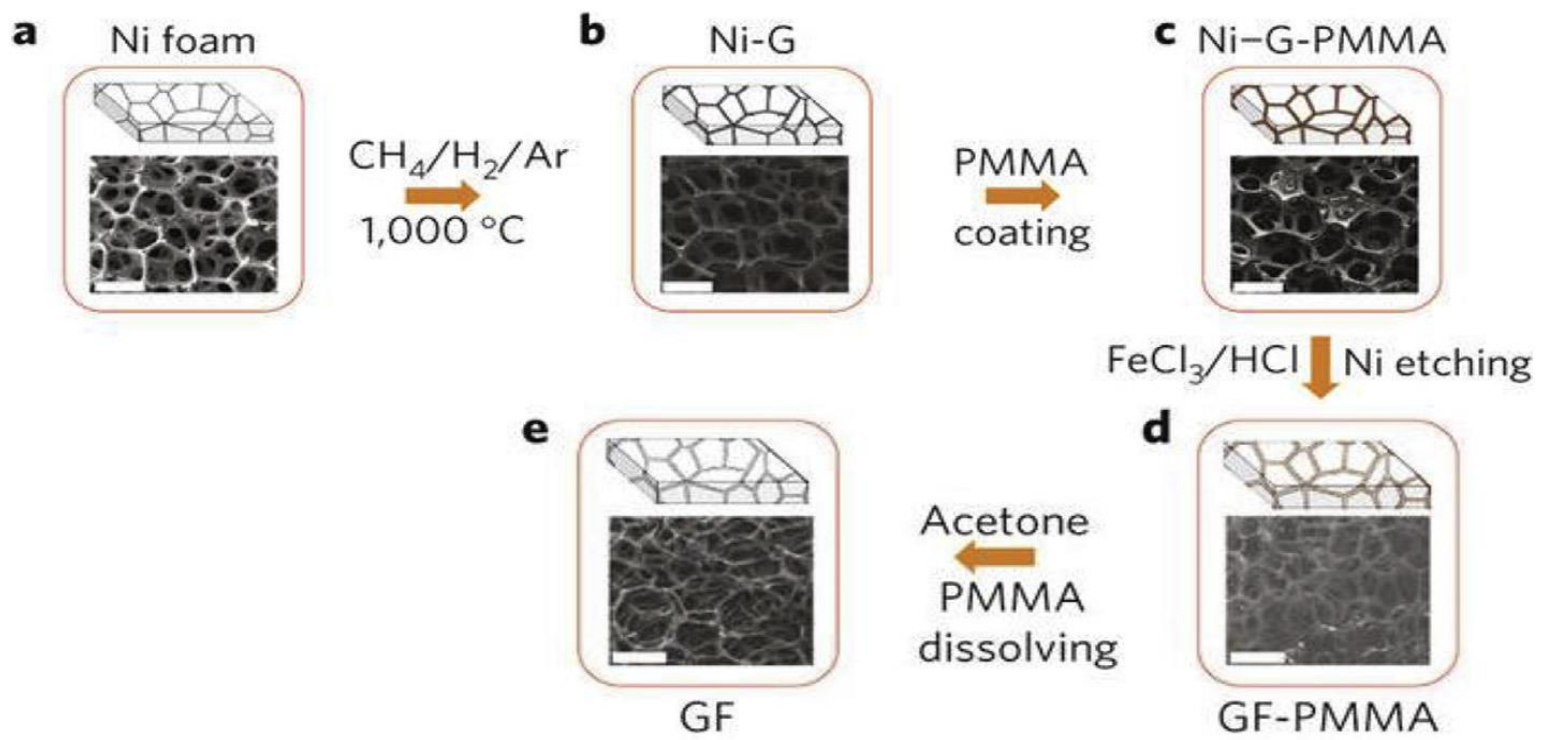

Figure 3: Synthesis of Graphene foam: ( $a$ and b) CVD growth of graphene films (Ni-G, b) using a nickel foam as scaffold. (c) as-grown graphene film after coating a thin PMMA supporting layer (Ni-G-PMMA). (d) Graphene foam coated with PMMA (GrF-PMMA) after etching the nickel foam with $\mathrm{FeCl}_{3} / \mathrm{HCl}$ solution. (e) Free-standing $\mathrm{GrF}$ after PMMA dissolution with acetone [67].

\subsubsection{Free Standing Graphene Foam Properties}

GrF macroporous framework offers promising properties that can be harnessed by polymer-based systems. Studies have shown that GrF node-branch anatomy plays a pivotal role in the mechanical behavior of free standing of GrF and GrF-based polymer composites [34]. GrF architecture exhibits structural coherence to effectively transfer load throughout 
the framework during its tensile deformation. Furthermore, comparison of GrF elastic modulus in tension and compression are evaluated using nanoindentation technique [34]. GrF wall tensile elastic modulus is four order of magnitude greater than when its compressive modulus. The remarkable elastic modulus can be ascribed to high in-plane mechanical behavior of graphene sheets during tension. Presence of deformation mechanisms could also contribute to the impressive mechanical properties of GrF. Deformation mechanisms such as GrF branch flexibility [70] and crack deflection [71]; vibration effect of GrF hollow wall [72]; sliding, rotation and rippling of graphene sheets can enhance the energy absorption, fracture toughness, damping behavior of GrF.

Schematic illustration in figure 4. also conveys insights into deformation of GrF in multiscale dimensions. It provides spatial and temporal understanding by featuring defects initiation, defects propagation and failure strain in multi-scale dimensions. In agreement with Nieto et al.'s investigation on GrF deformation, Pan. D et al. investigated uniaxial tensile behavior and fracture mode of 3D GrF expanded from 2D graphene mesoscopic model (figure 5) [73]. 2D graphene meso-structure transformation to 3D GrF points out the physical mechanisms during GrF tensile deformation. The mechanisms include squeezing of graphene flakes and formation and fading of graphene ripples, as tensile strain increases. The bonds breakage noticed between graphene flakes can be ascribed to rotation and slippage of graphene flakes. These mechanisms justify for the ductile fracture display of 3D GrF. Further investigation on mechanics of 3D graphene assembly elucidate that 3D graphene has outstanding high strength at comparably high density. In order words, its strength is 10 times as high as mild steel while its density is $4.6 \%$ of mild steel density [74]. 
Free standing GrF can also exhibit additional properties such super-hydrophobic properties [75], electrochemical characteristics [76] and microwave absorption properties [77].

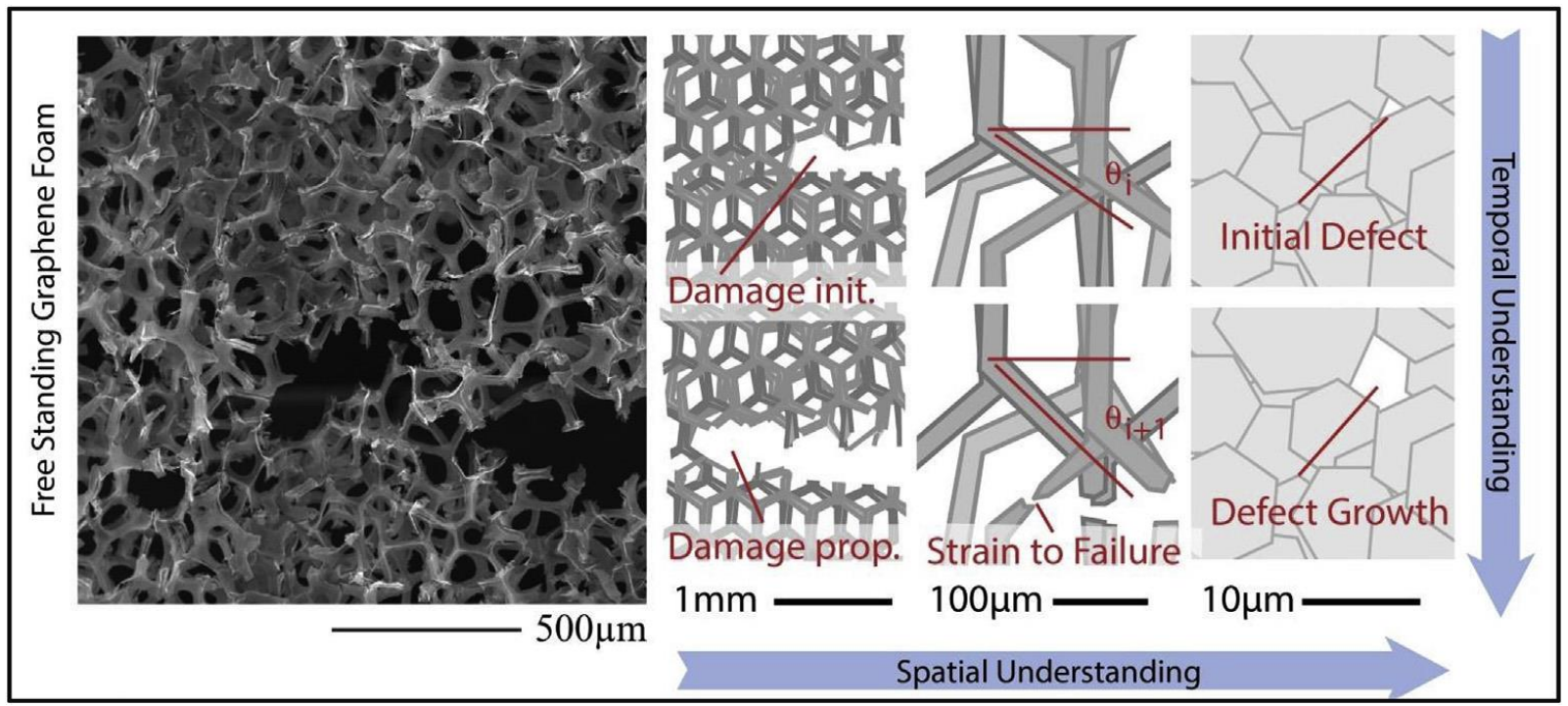

Figure 4: Insitu, multiscale, and dynamic analysis for determination of properties and failure mechanisms of free-standing $\mathrm{GrF}$ [23]. 


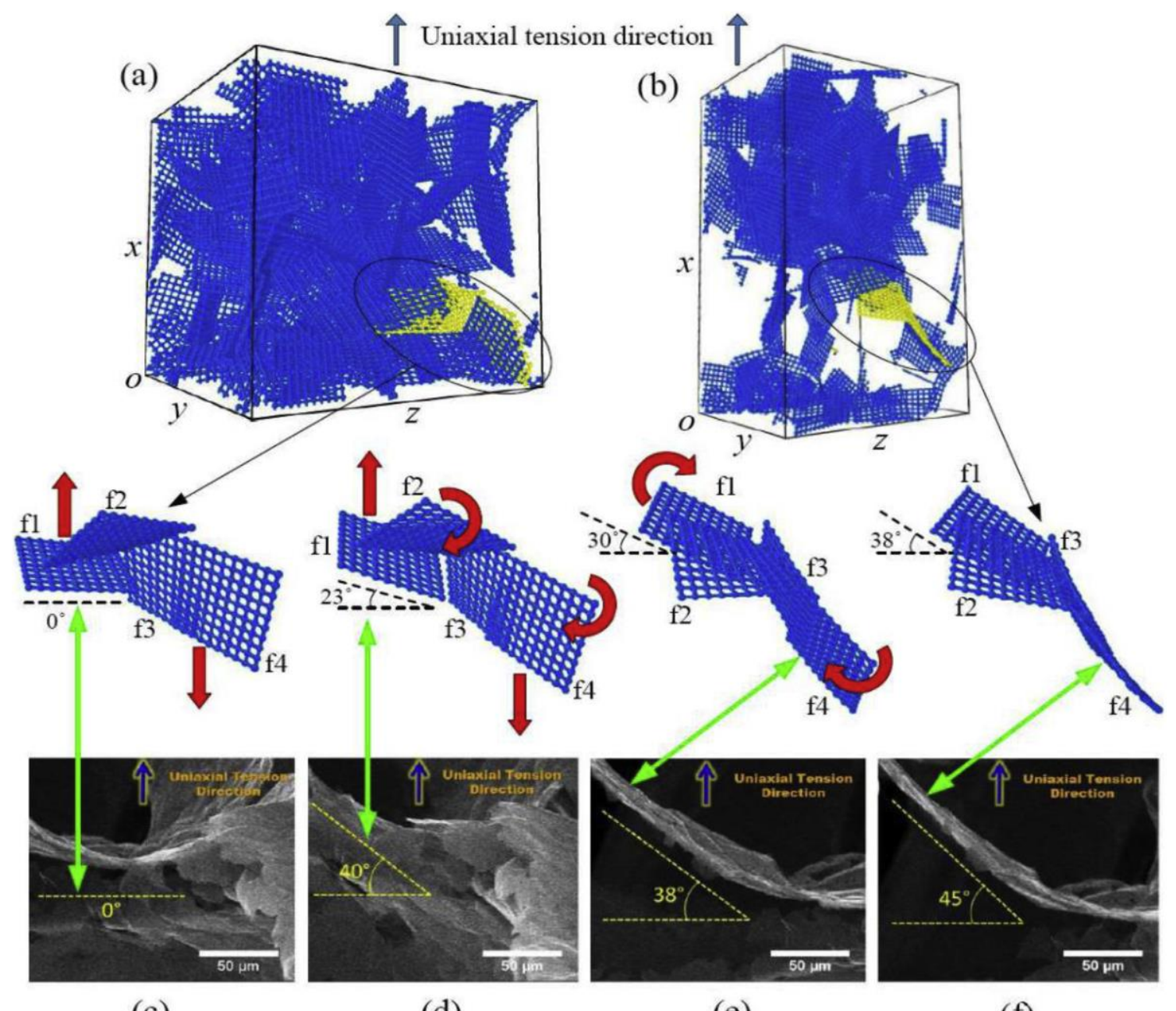

(c)

(d)

(e)

(f)

Figure 5: Meso-structure evolution during tensile deformation. (a) Beginning state of $\mathrm{GrF}$ in uniaxial tension (b) End state of GrF uniaxial tensile deformation; Yellow color represents local graphene flakes. (c-f) four sequential thermodynamic states of mesostructures during tensile deformation [73].

\subsubsection{Processing of Graphene Foam-Based Polymer Composites}

The structure-property behavior of GrF-based polymer matrix composites (GrFPMC) relies on its fabrication approach. The types of fabrication techniques for GrF-PMC include dip coating, casting, electrostatic spray deposition, vacuum infiltration, natural sedimentation and centrifugal assisted method. 


\subsubsection{Dip coating}

Dip coating technique is a suitable processing approach when liquid polymer precursor as the matrix for the fabrication of the composite. The method basically involves the immersion of pristine GrF in the liquid polymer and varying the parameters that would determine the quality and formation of the coating and composites, respectively. Such parameters include dipping time and $\mathrm{GrF}$ content with the variation in time of dipping resulting in different thicknesses in the coating.

Curing of the polymer-GrF system takes place under specific time and temperature conditions after the completion of the dip coating. Figure 6 presents a schematic showing dip coating process of graphene foam in the polymer. Figure 6 shows the gradual dipping and holding of GrF in the liquid polymer, using a control apparatus, respectively. After complete immersion of $\mathrm{GrF}$, the polymer-GrF is removed from the precursor. It is finally cured using UV light or heat source in Figure 6. The technique was adopted by Embrey et al. to fabricate epoxy-GrF multifunctional composite, where the composite was successfully synthesized [78].

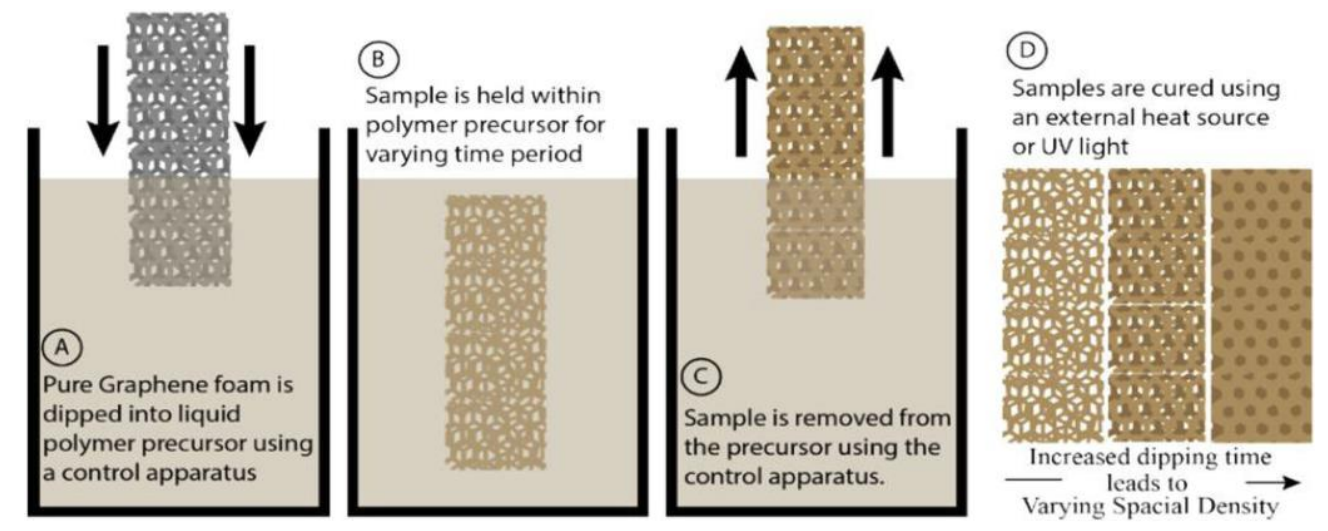

Figure 6: Schematic showing dip-coating process involving polymer-GrF coating process [23]. 


\subsubsection{Casting approach}

Casting is another fabrication method used in processing GrF-reinforced polymer composites. It involves pouring the polymer into a mold containing GrF. This technique allows GrF to be completely infiltrated with the polymer solution. The polymer infiltrates through the pore and coats the nodes and branches of the GrF. Subjected to heat source, the polymer containing the embedded GrF polymerizes and forms a resultant GrF based polymer composite [78].

\subsubsection{Electrostatic spray deposition}

Electrostatic spray deposition technique has also been adopted for the fabrication of carbon-based composites. Bakshi et al. successfully employed this approach in the synthesis of ultrahigh molecular weight polyethylene-carbon nanotube composites (UHMWPE-CNT) [79]. Since CNT and GrF belong to the same carbon filler family, electrostatic spray can be employed to fabricate GrF-based composites.

Electrostatic spray uses spraying technique to deposit polymer matrix in the powder form on the $\mathrm{GrF}$ for the formation of GrF-based polymer composites. Figure 7 demonstrates electrostatic spray coating deposition process. The GrF connects to an electrically conductive metal foil, which aids in the capture of the sprayed powder particles. Then, the spray gun applies an electrostatic charge to the polymer powder particles that attract to the GrF substrate. The polymer powder forms a uniform thin layer on GrF. Processing variables of spray time and distance can be tailored to obtain specified thickness of polymer deposition on the GrF. The polymer powder particles are in solid state before being put in the oven for melting and curing. After curing, the polymer forms a continuous 
thin layer on GrF if it has a good wettability with GrF. Curing of these components at its curing time and temperature produces consolidated GrF-PMC composite.
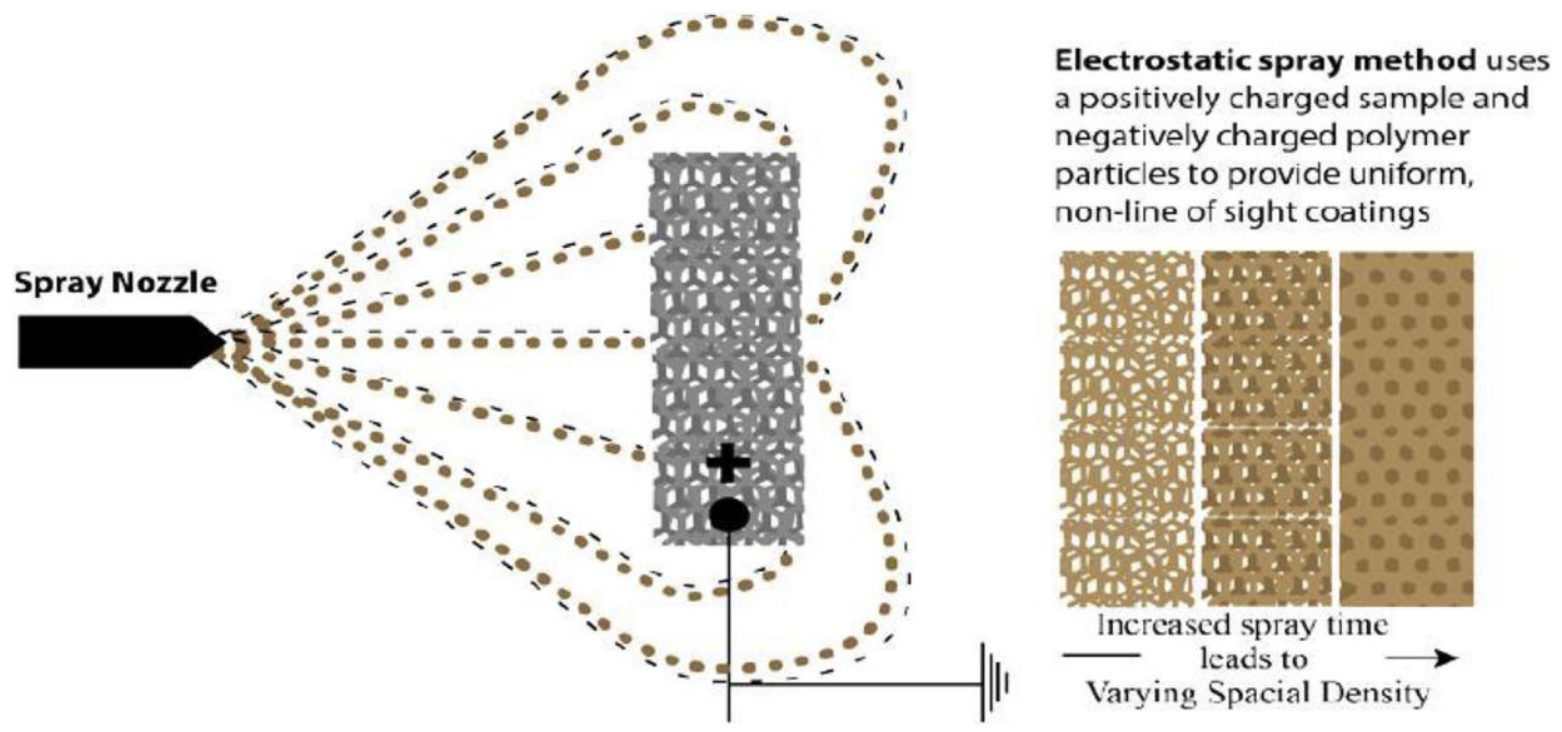

Figure 7: Schematic of electrostatic spray coating process [23].

\subsubsection{Vacuum infiltration technique}

Vacuum infiltration approach can be used in the development of GrF-based polymer composites. This technique involves infusion of the polymer into GrF inside a vacuum chamber. It has been adopted in the fabrication of GrF-based polydimethylsiloxane (PDMS) composite [80]. Consolidation of PDMS with GrF takes place under vacuum, where the PDMS infiltrates through the macroporous architecture of the GrF, as air pulled out from the vacuum chamber. This is thereafter cured at $80^{\circ} \mathrm{C}$ to form a solid GrF-PDMS composite. 


\subsubsection{Properties of Graphene Foam-Based Polymer Composites}

\subsubsection{Mechanical Properties}

Noticeable enhancement in the mechanical behavior of GrF-based polymer composites can be traced to synergistic contribution from the polymer and GrF. Examples of polymer solutions which are integrated with GrF for improvement in their mechanical properties include PDMS [81]-[84], epoxy [71], [78], [85], polyimide [70], polyurethane [86], polylactic acid-poly $\varepsilon$ caprolactone [87]. Synthesis methods of GrF-based polymer composites discussed in the preceding section are important to achieve high performance in the properties of these polymers. Schematic in figure 8. portrays the processing (either dip coating or casting) of GrF-PMC in which interface defects such as delamination could occur. However, occlusion of the defect growth happens after loading; defects are filled owing to the hierarchical structure of GrF in the polymer matrix.

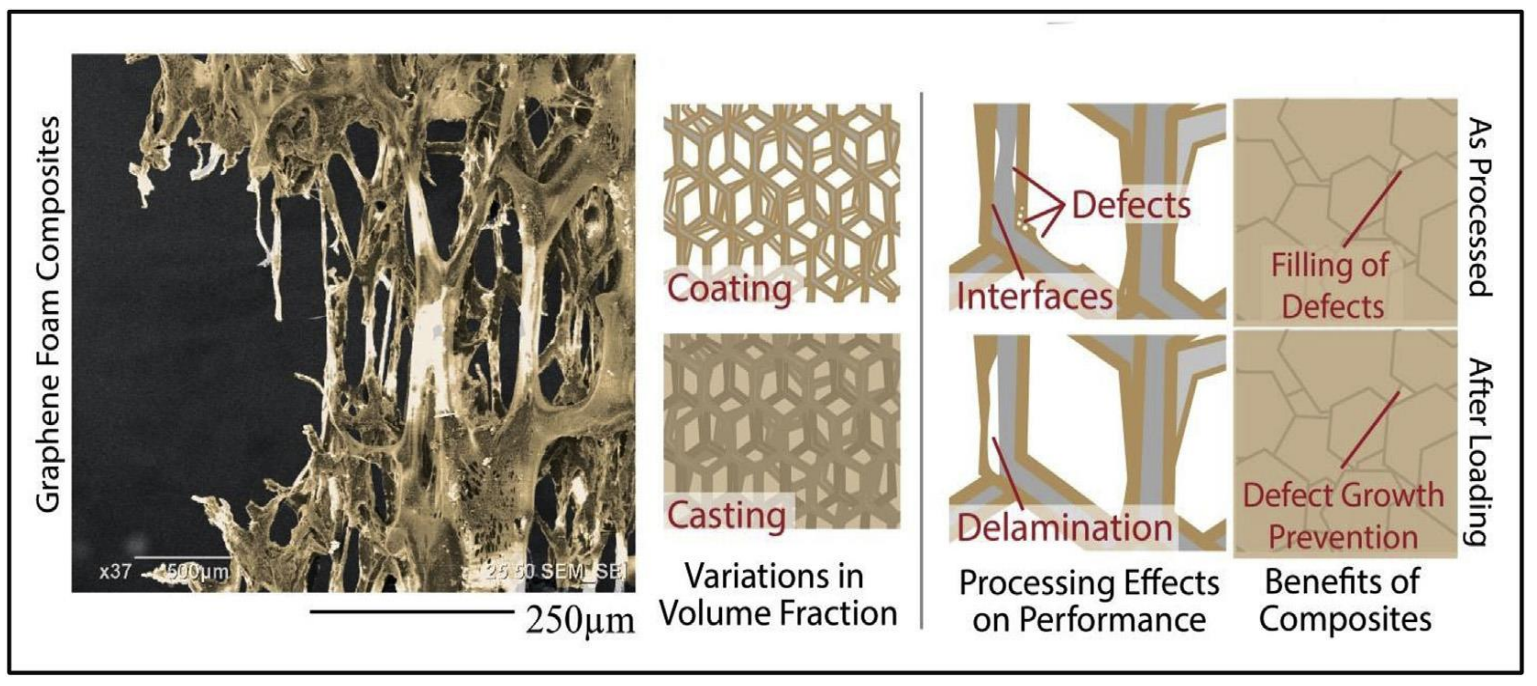

Figure 8: Schematics showing failure mechanisms and influence of processing on the behavior of graphene foam-based polymer composites fabricated by coating and casting [23]. 
Hierarchical GrF architecture allows load transfer between the matrix and nodebranch framework of GrF. This consequently results in the polymer infiltrating the interface defects or delaminated areas, during and after loading. It thereby leads to defect closure in GrF-based polymer composites. Impressive GrF mechanical behavior as highlighted section 2.2.2 positively impact polymer composites. Addition of 0.13 and 0.6 wt. $\%$ of GrF results in $12 \%$ increase in ultimate tensile strength and $10 \%$ enhancement in the flexural stress of the epoxy composites, respectively [78]. K. Siva et al. also showed the boost in mechanical properties (elastic modulus, peak stress, and absorption energy) of GrF-PDMS compared with GrF, carbon nanotube (CNT) and CNT/PDMS [83]. The tuned porosity and continuous GrF network, which fosters stress transfer, could be attributed for these improvements. GrF role in flexible, stretchable, and sensitive strain sensors has also been displayed in GrF/PDMS and fragmented GrF/PDMS composites. Investigation on GrF/PDMS composite as strain sensor exhibited a high gauge factor of 98.6 under 5\% applied strain. It was also 30\% stretchable of its original length [88]. The mechanism behind the strain sensor behavior during stretching is graphene sheets slippage. The slippage makes cracks to occur due to breaking of the pi-piconjugated interaction within the graphene sheets. Crack reconnection is the mechanism that occurs during relaxation cycles. Fragmented GrF/PDMS has higher sensitivity with a gauge factor range 15-29 than GrF/PDMS with a gauge factor 2.2 [89]. This can be attributed to its resistance change increase owing to large contact area between the adjacent fragmented graphene foam. Fragmented GrF/PDMS also has high durability and stretchability of over $70 \%$ strain and more than 1000 stretching-releasing cycles. Another investigation on GrF/PDMS displayed improvement in its Young modulus, tensile and compressive strains. The improvement was 
ascribed to brilliant mechanical properties of $\mathrm{GrF}$ [90]. GrF addition to PDMS in figure 10 displayed peak modulus of GrF/PDMS (50 MPa) compared with pristine graphene foam (8.57 MPa) in figure 9. Figure 10 points out the synergistic influence of GrF and PDMS on the polymer composite.

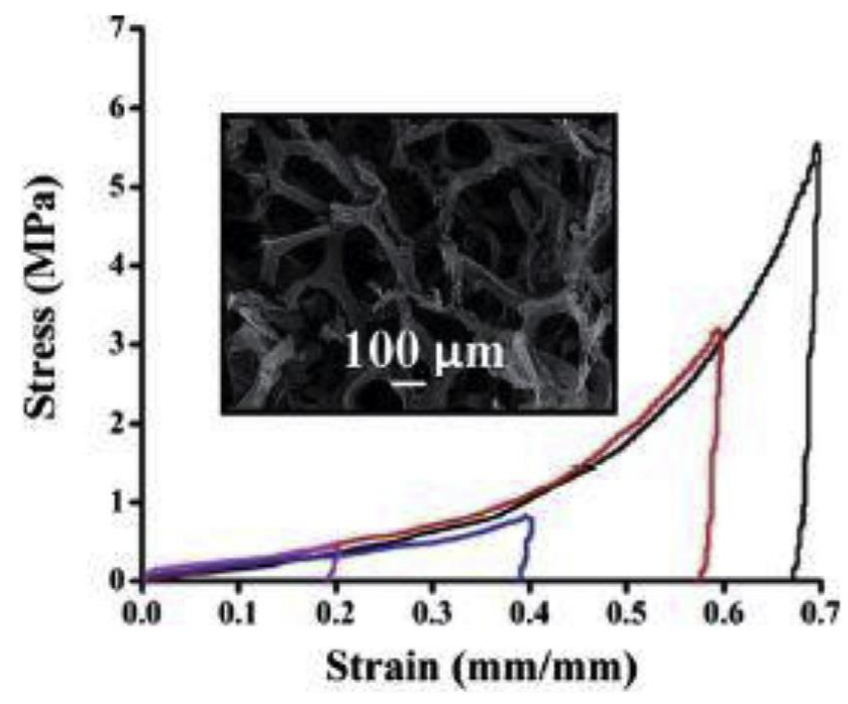

Figure 9: Stress-strain graph of Graphene Foam. i) black line - 70\% loading strain; ii) red line $-60 \%$ loading strain; iii) blue line e $40 \%$ loading strain; iv) purple line $-20 \%$ loading strain [83].

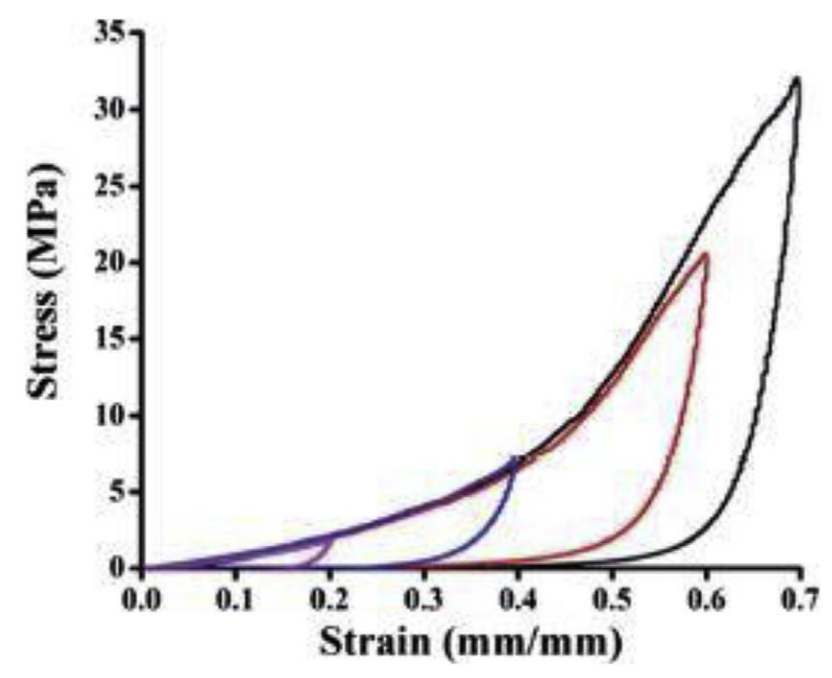

Figure 10: Stress-strain graph of GrF/PDMS. i) black line - 70\% loading strain; ii) red line $60 \%$ loading strain; iii) blue line $40 \%$ loading strain; iv) purple line $20 \%$ loading strain [83]. 
Polylactic acid-poly $\varepsilon$ caprolactone (PLC) and polyimide (PI) are among other polymers that could also serve as matrices in GrF-based composites. A. Nieto et al. explained that GrF-PLC could be a robust support-structure for human mesenchymal stem cells (hMSCs). This is due to superior strength and ductility of the GrF-PLC scaffold. The bio-mimetic mechanical properties of GrF-PLC, coupled with the ability to support human stem cells differentiation, makes it suitable for tissue engineering applications such as growth of cartilage extracellular matrix (ECM) [87]. Furthermore, Nautiyal et al. demonstrated how mixture PI and GrF could exhibit remarkable damping properties [70]. The work reveals $300 \%$ enhancement in the damping characteristics of PI (0.12) after $1.5 \mathrm{wt}$ \% GrF addition at room temperature. Impressively, the damping capacity of PI-GrF (0.36) is retained after its exposure to high temperature $\left(4000^{\circ} \mathrm{C}\right)$ which is $\mathrm{Tg}$ of PI. It portrays that PI-GrF nanocomposites are desirable for exposure to extreme thermal conditions experienced by aircrafts and space vehicles. All these studies point to the impact of graphene foam reinforcement on the mechanical properties of polymer composites.

\subsubsection{Electrical properties}

The synergy of polymers and GrF nano-reinforcement has demonstrated boost in the electrical behavior of 3D GrF-based polymer composites. In fact, GrF excellent electrical properties have received credit for improvement in the electrical conduction of polymer composites. For instance, Jia et al. probed into the electrical characteristics of GrF-epoxy composites [71]. His group observed excellent electrical conductivity of $3 \mathrm{~S} / \mathrm{cm}$ after 0.2 wt. \% GrF addition. The electrical performance can be ascribed to seamless pathway of $\mathrm{GrF}$ network for charge carriers. Similarly, G. Chen et al. noticed the output of 2.5 vol.\% GrFepoxy composite. The composite displayed remarkable peak conductivity of $196 \mathrm{~S} / \mathrm{cm}$ 
which points to the GrF inherent capactity to significantly enhanced electrical properties of the polymer composites [91].

Different from GrF-epoxy investigation, X. Sun et al. conducted a study on electrical conductivity of GrF/PDMS and GrF/CNT/PDMS [92][]. The result showed that GrF/PDMS and GrF/CNT/PDMS exhibit enhanced electrical conductivity values of 6.74 and $31.5 \mathrm{~S} / \mathrm{cm}$, respectively. Increase in graphene and CNT concentration results in complete percolation in PDMS. The complete percolation facilitated PDMS to display more electrical conductivity. It indicates that effective synergy between CNT and GrF electron conduction occured. This portrays $\mathrm{GrF}$ as potential filler that could efficiently mingle with other carbon filler. Y. Jun et al. also examined study of in-plane and throughplane conductivities of GrF/PDMS. The GrF were made from large and small graphene oxide foams (graphene flakes) [93]. Comparison of the in-plane electrical conductivity of the GrF/PDMS (graphene flakes) and GrF/PDMS was provided. Results, as depicted in figure 11a, showed that lower graphene content for large graphene flakes gives higher inplane conductivity than the standard conductivity. This can be attributed to the bigger size of graphene sheets. Its large size, in addition to its network structure, creates more surface area for efficient pathway for electron transfer. Figure $11 \mathrm{~b}$ also illustrates the behavior of through-plane conductivity and normal conductivity of GrF/PDMS. It was observed that the through-plane conductivity was higher than the average conductivity of increased graphene content for small graphene flakes. This could be due to more electron percolation through increased content of the small graphene flakes. It can, therefore, be attested that GrF infiltration with polymers enables the tailoring and improvement of electrical behavior of $\mathrm{GrF} / \mathrm{PDMS}$ composites. 

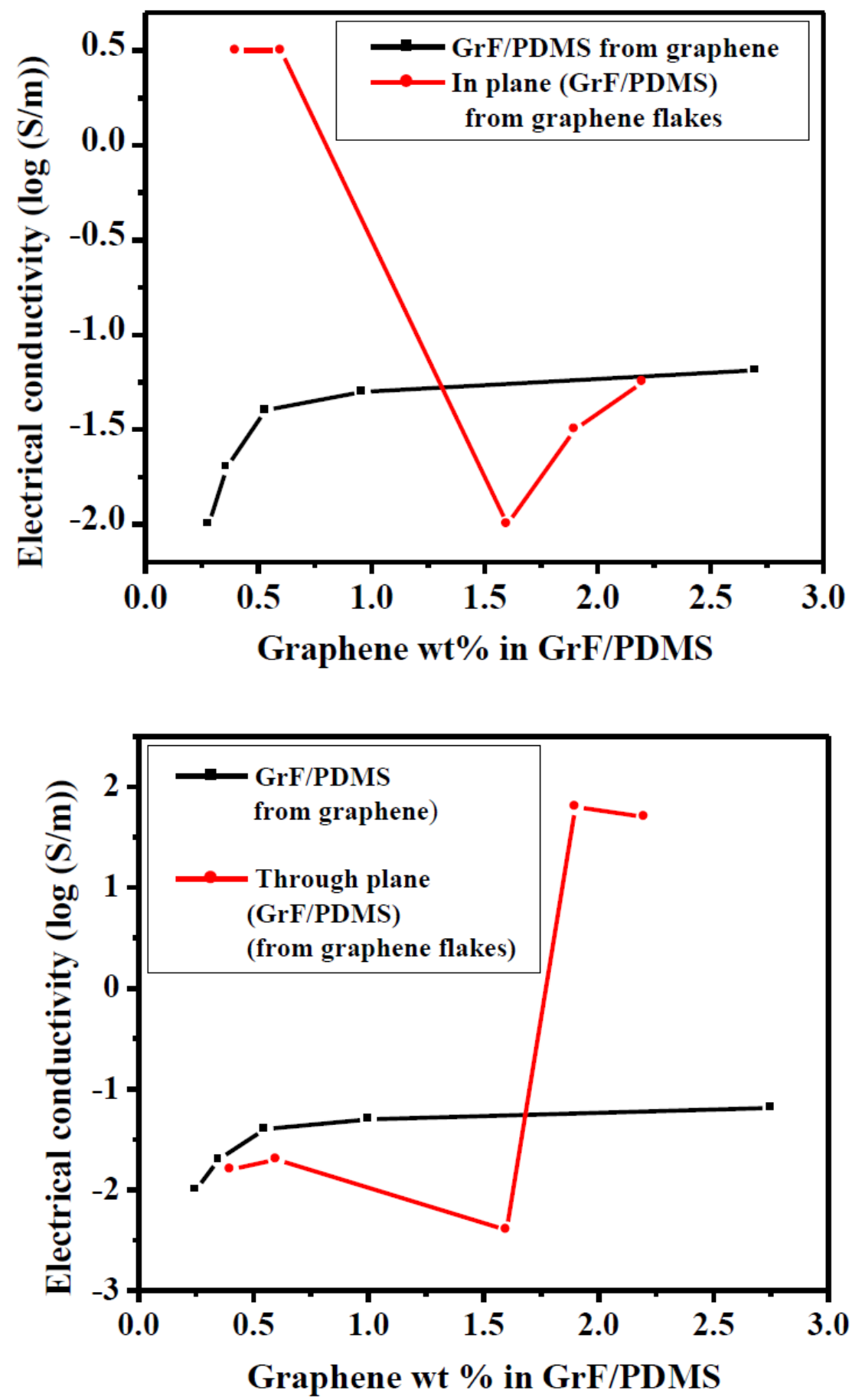

Figure 11: (a). Electrical conductivities of GrF/PDMS (in-plane) vs Graphene wt.\%. (b) Electrical conductivities of GrF/PDMS (through-plane) vs Graphene wt.\% [23].

Furthermore, figure 12 shows the comparison of electrical conductivity behaviors of GrF/PDMS [92] and GrF/Epoxy [93]. Starting at 0.5 wt.\% GrF loading, GrF epoxy 
composite has higher conductivity than GrF-PDMS composite. It points out that below 0.5 wt.\% filler loading, GrF does not bring about adequate percolation in the epoxy matrix for appreciable electron conduction. Electrical conductivity increases above 0.5 wt.\% GrF. It is also noticed that after 0.6 wt.\% of GrF filler in PDMS, GrF attained its percolation threshold. This is revealed in the steady electrical conductivity value of GrF-PDMS composite, as shown in figure 12. Meanwhile, GrF-epoxy composite attained percolation limit after 2.0 wt.\% GrF loading in epoxy (more than thrice of GrF loading in PDMS). This could be due to the viscosity behavior of epoxy. Epoxy viscosity, which is higher than that of PDMS, requires more GrF loading to arrive at its percolation threshold. It thus attests that choice of polymer matrix can considerably influence the electrical conductivity of the GrF reinforcement in polymer matrices. More importantly, electrical property of GrF in epoxy and PDMS helps in tailoring and improving their composites.

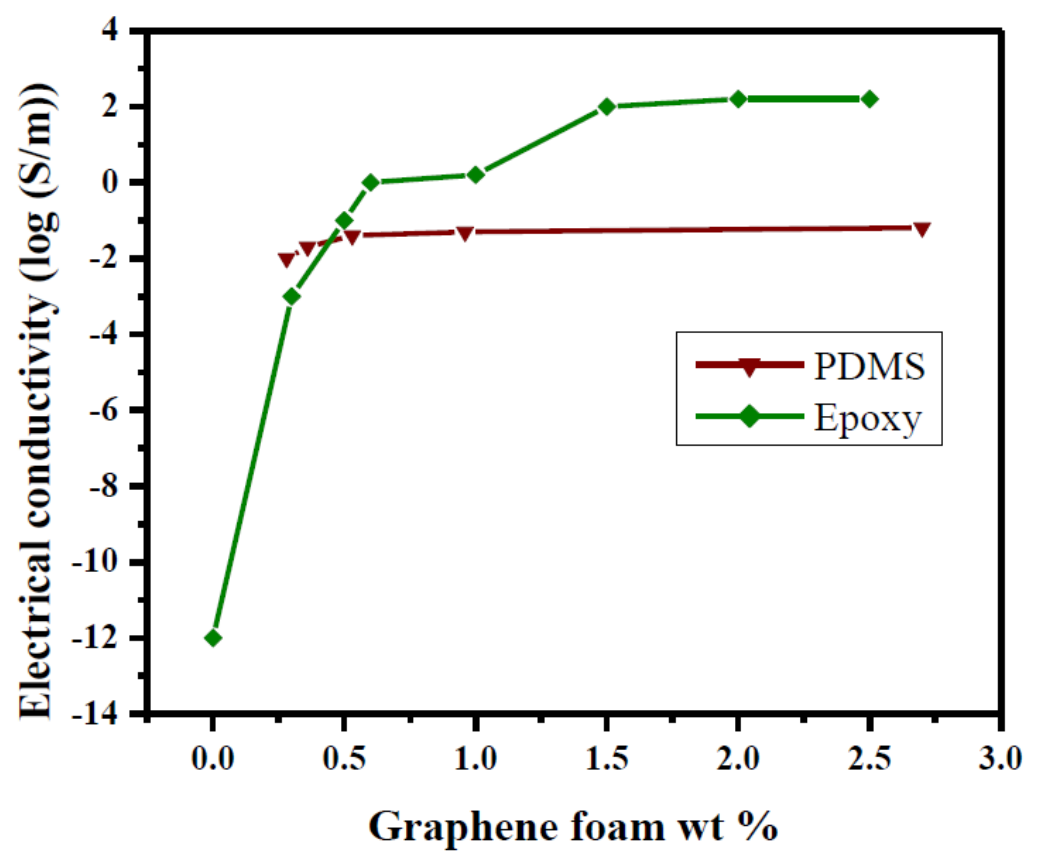

Figure 12: Electrical conductivities of GrF/PDMS \& GrF/Epoxy vs Graphene foam wt.\% [23]. 
GrF incorporation in other polymers, such as polyvinylidene fluoride (PVDF), polycaprolactone (PCL) and polypyyrole (PPy), have also shown noticeable improvement in their electrical properties. PVDF/GrF \& PCL/GrF composites scaffolds indicate that their electrical conductivities were maintained at $0.07 \pm 0.02 \mathrm{~S} / \mathrm{cm}$ and $0.13 \pm 0.06 \mathrm{~S} / \mathrm{cm}$, respectively compared with pristine $\mathrm{GrF}$ conductivity $(0.36 \pm 0.11 \mathrm{~S} / \mathrm{cm})$. The ability to retain such conductivities was ascribed to the free flow of electrons from the electrodes to the graphene foam network due to micro cracks within the polymer coatings [94].

Besides, results from GrF-PPy composites pointed to remarkable cycle life of GrF and PPy- GrF electrodes. Initial capacitances are preserved after 10000 and 6000 chargedischarge cycles, respectively. It was touted that the hierarchical structure of GrF and its density on the flexible electrode are responsible for the enhanced capacitance stability [95]. Research on polyimide and polyaniline nanofiber sponge (PANI-NFS) composites substantiated GrF as electrical reinforcement. M. Loeblein et al. asserted that 3D grapheneinfused polyimide (PI) could exhibit a higher electrical conductivity of large 10 orders of magnitude than PI [96]. Also, examination of PANI-NFS/GrF conductivity displayed conductance $(>10.8 \mathrm{~S} / \mathrm{cm})$ greater than normal PANI-NFS $(3.5 \mathrm{~S} / \mathrm{cm})$ [97]. The outcome was due to efficient electronic transport of GrF scaffold having a pore size of 100-500 $\mu \mathrm{m}$. GrF hierarchical pore structures when combined with PANI-NFS (100-500 nm pore size) contribute to extremely high volumetric and gravimetric capacitances of the composite. Overall, improvements in electrical behavior of the composites were primarily due to the seamlessly interconnected porous structures of the graphene foam, which supplied more efficient path for electron transfer inside the polymers. 


\subsubsection{Thermal behavior}

Numerous studies have investigated distinct thermal properties of some filler materials, such as graphite, carbon nanotube, in polymer matrices. These reinforcing agents have accounted for enhancement in the thermal performance of polymer nanocomposites [81], [98], [99]. Recent research works on impregnation of polymers with graphene foam have demonstrated greater thermal property enhancements of the polymer composites. The scheme in figure 13 portrays thermal-electron transport process that normally occurs in polymer matrices reinforced with GrF, 2D, and 1D carbon-based nanomaterials. Phonons and electrons are galvanized from a heat source which radiates into the carbon-based composites. It results in thermal energy transfer, with GrF showing consistent performance in electronic thermal conduction within the composites.

Thermal-electron transport in Carbon based nanomaterials

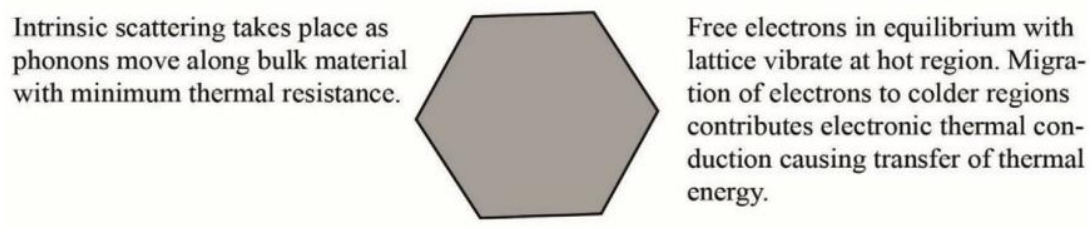

Thermal-electron transport in Composites reinforced with Carbon-based nanomaterials
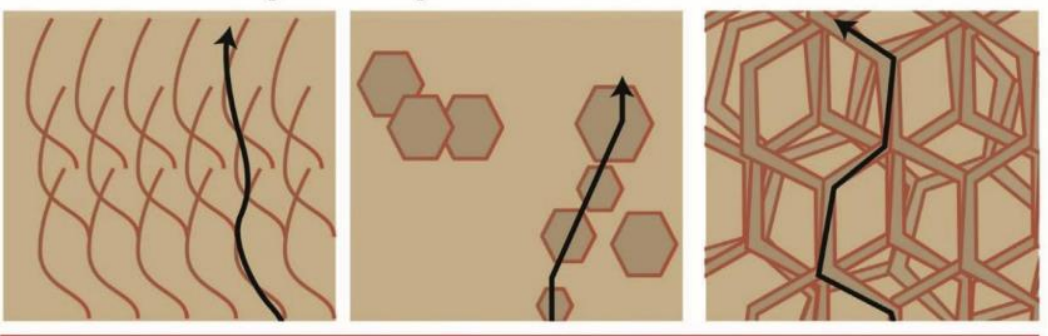

Heat Source

1D Nanomaterials and 2D Nanomaterials

Graphene Foam

Thermal-electron transport requires alignment/disper-

Alignment and dispersion sion, high volume fraction. Otherwise dominated by matrix properties.

through foam structure allows

for low volume fraction and

Inconsistent performance batch-to-batch

isotropic bulk response.

Consistent performance

batch-to-batch

Figure 13: Scheme of thermal and electron transport in composites reinforced with 1D, $2 \mathrm{D}$ and $3 \mathrm{D}$ GrF [23]. 
Pettes et al. observed increase in $\mathrm{GrF}$ thermal conductivity from 0.26 to $1.7 \mathrm{Wm}^{-1} \mathrm{~K}^{-}$ ${ }^{1}$ after using different etchants for nickel foam [100]. The thermal conductivity of pure GrF improved as the temperature increased above room temperature [101]. GrF also has a low thermal interfacial resistance of $0.04 \mathrm{~cm}^{2} \mathrm{KW}^{-1}$, which is ten times lower than conventional thermal paste and grease used as thermal interface materials [102]. With its unique thermal properties, combination of GrF reinforcement with polymers (mostly PDMS) [81] has great potential to enhance thermal properties of polymer composites. GrF/PDMS composite has very low thermal resistance $14 \mathrm{~mm}^{2} \mathrm{KW}^{-1}$ compared with commercial polymer metal composite (silver particles in epoxy) [103]. This is due to the interconnected distributed structure of GrF [104]. Some studies have probed GrF thermal behavior infiltrated with polyimide and polyamide [96], [105]. Displayed in figure 14, bare Polyimide thermal conductivity $\left(0.2 \mathrm{Wm}^{-1} \mathrm{~K}^{-1}\right)$ received a boost in its conductivity $\left(1.85 \mathrm{Wm}^{-1} \mathrm{~K}^{-1}\right)$ at $150^{\circ} \mathrm{C}$ after mere 0.1 wt. \% GrF addition. It suggests that the node-branch network of GrF allowed increased phonon flow within the PI/GrF composite. Furthermore, the comparison of PDMS/GrF and PI/GrF highlighted that PI/GrF possessed higher thermal conductivity and stability than PDMS/GrF composites. This could be due to smaller interface area exposure of PI/GrF to air, unlike that of PDMS. 


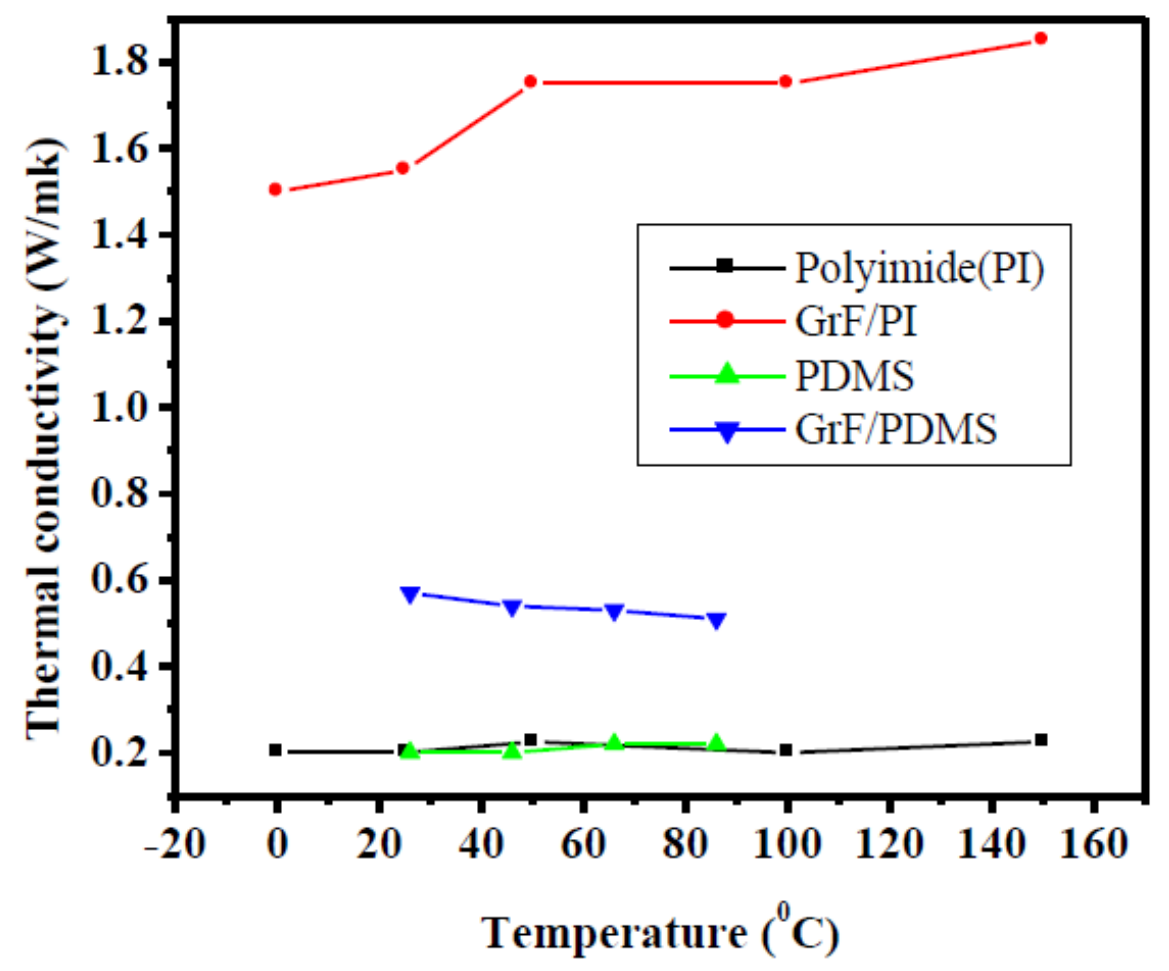

Figure. 14: Graph of thermal conductivity vs temperature of PI and 0.35 wt. $\% \mathrm{GrF} / \mathrm{PI}$ at $150^{\circ} \mathrm{C}$, and PDMS and $0.7 \mathrm{wt} . \% \mathrm{GrF} / \mathrm{PDMS}$ at $85^{\circ} \mathrm{C}$ [23].

Figure 15 presents thermal conductivity behavior of polymer composites with varying $\mathrm{GrF}$ and flakes filler contents. Polyamide/GrF [105] displayed better thermal conductivity than multi-graphene flakes (MGf) in PDMS [106]. This indicates that hierarchical structure of $\mathrm{GrF}$ is conducive for thermal conduction. Interestingly, the combination of MGf and GrF in PDMS (with a small increase in GrF content) gave higher thermal conductivity values compared to that of more GrF content in polyamide. The combined effort of GrF with other filler materials, such as carbon fiber (CF), carbon black (CB) and graphene flakes, has also stepped up the thermal behavior of polymer nanocomposites. This is due to large pathways of heat conduction made by the connection of $\mathrm{CB}$ and $\mathrm{CF}$ with the $\mathrm{GrF}$ branches. It was observed that the loading of 8 wt.\% $\mathrm{CB}$ into PDMS/GrF ( 0.5 wt. $\%)$ enhanced the thermal conductivity by $72 \%$ in comparison to 
PDMS/GrF (figure 15) [107]. When GrF and CF are combined in PDMS matrix, it also revealed appreciable improvement in the thermal behavior of $\mathrm{CF} / \mathrm{GrF} / \mathrm{PDMS}$. There are $41 \%$ and $162 \%$ increase in thermal conductivity of CF/GrF/PDMS than in GrF/PDMS and PDMS, respectively. It suggests that formation of a combined conductive network of CF within and GrF could have accounted for the significant thermal conductivity enhancement. These results thus indicate that GrF could synergize excellently with $\mathrm{CB}$ and co-contribute electron transfer with $\mathrm{CF}$.

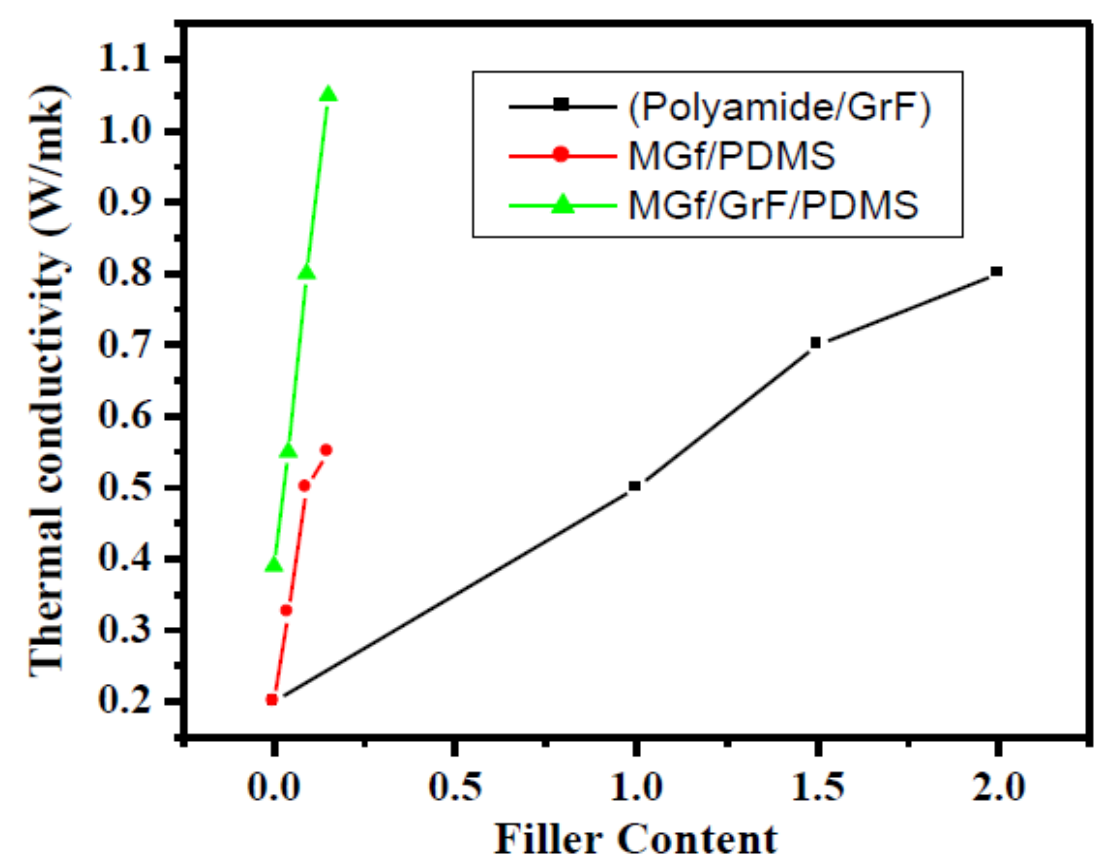

Figure 15: Graph of thermal conductivity vs filler content of Polyamide/GrF, Multigraphene flakes/PDMS, Multigraphene flakes/GrF/PDMS [23].

\subsubsection{Additional Properties of Graphene Foam}

GrF-reinforced polymer composites can exhibit additional properties including biological, acoustic, and chemical. A.Nieto et al. examined biocompatibility and bio-tolerant properties of GrF with copolymer when used as a scaffold in the culturing of human 
mesenchymal stem cells (hMSCs) [87]. Cellular studies conducted showed that the hMSCs survived and proliferated on the GrF reinforced composite. In another study, polydopamine PDA/GrF composite was synthesized for enzyme immobilization. GrF served as a bioelectrode in the immobilization of horseradish peroxidase [108]. A recent study discovered that GrF/Tungsten (W)/Epoxy composite could produce improved acoustic performance [85]. GrF hierarchical and mesoporous structure is employed in the epoxy composite to provide a confined space that would allow dense packing of the tungsten spheres within GrF pores. The compactness among epoxy, tungsten spheres, and GrF would result in a reduction of air that can propagate acoustic wave. This would lead to high acoustic impedance and increased acoustic attenuation, which is required for excellent backing material. Corrosion behavior of 3D GrF-nickel foam polymer after exposure to $3.5 \% \mathrm{NaCl}$ was also examined. It displayed decreased corrosion with increasing graphene thickness [109]. The observation suggests that when graphene film applies itself as corrosion protective coating for long-term, its compactness is very essential. It implies that when the graphene is sandwiched between nickel and PDMS, it could impede ion or oxygen diffusion to the nickel surface if its thickness is increased. This would consequently result in decreased corrosion of the composite.

Based on this comprehensive review on $\mathrm{GrF}, \mathrm{GrF}$ has thus been able to enrich conventional polymers with its great properties. To broaden the horizon where GrF can serve as reinforcing agent in polymers, this study further examines advanced polymer systems namely shape memory polymers. 


\subsection{Shape Memory Polymer Epoxy}

Shape memory polymer (SMP) is a smart polymeric material that has great scientific and technological significance [110]. SMP has capability to retain a temporarily deformed shape and to restore the deformed shape to its permanent original shape when actuated by an external stimulus. As an intelligent system, it can respond to diverse stimuli. Such stimuli can be in form of magnetic [111], ultrasound [112], direct heat [113], indirect heat (electricity) [114], irradiation (light at different wavelengths) [115], chemical environment (pH, Water/solvent ions, redox conditions) [116]. However, most SMP are thermally actuated since they are engineered from traditional polymers which have temperaturesensitive properties.

SMP can be divided into two main categories: i) physically cross-linked SMP (thermoplastics) and ii) chemically crosslinked SMP (thermosets) [110] . Thermoset SMP are amorphous polymers. They have received more attention than thermoplastic SMP primarily due to its versatile application purpose [117]. A foremost example of thermoset SMP is SMP epoxy [118]. SMP epoxy is an amorphous polymer that can be made by polymerization of liquid monomers with crosslinkers. It consists of two different epoxy monomers and a curing agent. Typical epoxy resins include Diglycidyl ether bisphenol A (DGEBA) and Neopentyl glycol diglycidyl ether (NGDE). Jeffamine D230, decylamine, etc., serve as curing agents for the precursors. Chemical structures of the resins are shown in figure 16a-d: 


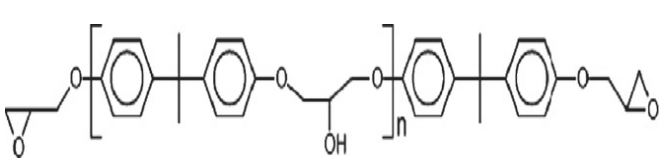

a)

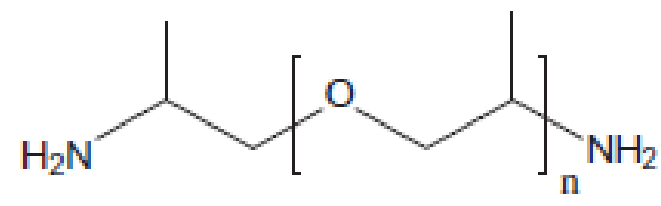

c)

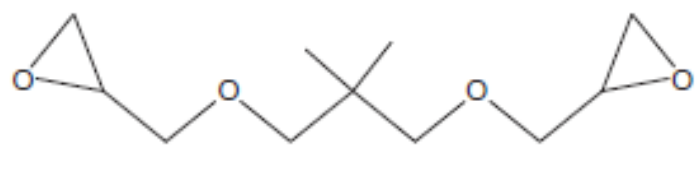

b)

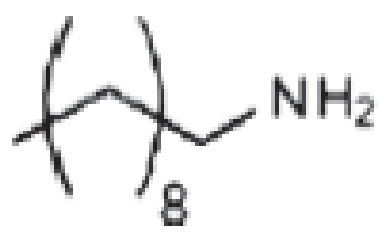

d)

Figure 16: Chemical Structures of SMP epoxy components (a) Diglycidyl ether bisphenol A (DGEBA); (b) Neopentyl glycol diglycidyl ether (NGDE); (c) Jeffamine D-230 (n 2.5); (d) decylamine [118].

The increasing interest in SMP epoxy can be ascribed to its good attributes. Such attributes include facile processing, low shrinkage during curing, availability of the monomers, good thermomechanical stability of the polymer and versatility in chemical structures [16]. Its structural flexibility allows it to have high recoverable strain and exhibit desirable shape memory performance. These attributes have made SMP epoxy to be considered for structural and other multiple applications [119]. Nevertheless, SMP epoxy has some inherent limitations that cannot be overshadowed by its advantages. Limitations include low elastic modulus $(1.4-2.5 \mathrm{GPa})$ [19], [20], poor thermal conduction $(<0.3 \mathrm{~W} / \mathrm{mk})$ [18], low recovery stress $(1-3 \mathrm{GPa})$ [21], and slow recovery time. Its low intrinsic stiffness accounts for low recovery stress generated during shape recovery. Poor heat transfer also leads to slow thermal diffusion through SMP epoxy. Low thermal diffusivity translates to longer recovery time for SMP epoxy to attain its permanent shape. It is believed that reinforcement addition to create SMP epoxy composites is a feasible solution [22]. The composites can 
propel SMP epoxy matrix to operate at optimum level of performance. Research efforts have thus been geared towards addressing the drawbacks in SMP epoxy using conventional inorganic and carbon-based fillers.

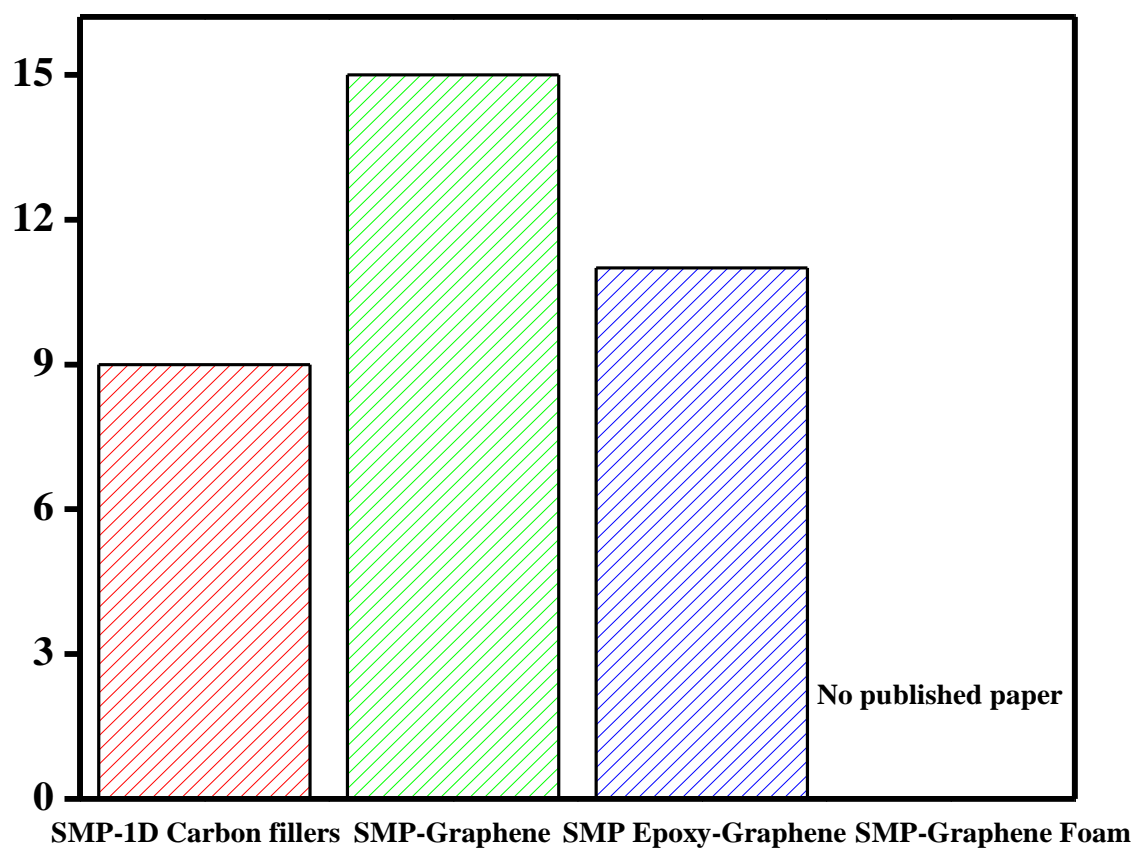

Figure 17: Number of publications on SMP reinforced with carbon-based fillers during 2015-date.

Figure 17 conveys the number of publications on carbon-based SMP composites and graphene based SMP epoxy composites. Compared with SMP-1D carbon fillers, SMPgraphene and SMP epoxy-graphene composites have increased number of publications. Though one-dimensional carbon fillers have brought a bit of improvement, their morphology (as stress initiators) and anisotropy property in the resultant composites could 
serve as drawbacks. This explains a few interests shown in them; hence, the shift to 2D graphene fillers. It construes that the drawbacks in 1D carbon fillers can be further addressed by reinforcing SMP epoxy with graphene-based fillers to boost shape memory performance of SMP epoxy.

\subsubsection{Graphene-Reinforced Shape Memory Epoxy Composites}

The brilliant properties of graphene have stirred up attention for it to be adopted as reinforcement in SMP epoxy composites. Its extraordinary high elastic modulus (1 TPa) has potential to boost recovery force of SMP epoxy [120]. High tensile strength of graphene can further improve the mechanical behavior of SMP epoxy composites. High thermal and electrical conductivity of graphene reinforcement is also a great advantage to SMP. It can help provide effective thermal stimulus, improving SMP actuation output [121]. Strong anisotropic bonding and low mass of carbon atoms can be attributed to its easy phonon flow behavior that supply heat conduction [122]. Graphene electrical property can also enable it serve as an alternative stimulus for non-electrically conductive SMP. The delocalized $\pi$ electrons within the conduction band accounts for electron flow in graphene sheet [122]. Based on the impressive highlighted properties, adopting graphene-based fillers is promising for SMP epoxy performance in structural applications.

\subsubsection{Synthesis of Graphene-Reinforced Shape Memory Epoxy Composites}

The preparation of SMP epoxy-graphene is grouped into two subsections. The first section focuses on graphene dispersion. The second section considers the processing of the SMP epoxy-graphene composites. These fabrication approaches are pivotal to propertyperformance characteristics of the composites. 


\subsubsection{Graphene Dispersion Technique}

It is important for graphene to be well dispersed for it to serve as an effective reinforcement in SMP epoxy. Graphene can be subjected to different dispersion methods. Typical dispersion approach used for graphene reinforcement in SMP is ultrasonication. Ultrasonication is simply a process that harness sound energy at high frequencies to break apart particle aggregates by cavitation, bubble expansion and implosion in the solvent [123].

Few studies have used this approach to prepare graphene filler for SMP. E. Wang et al. synthesized graphene oxide (GO) short fibers [124]. The GO fibers dispersed in deionized water with the aid of ultrasonicating machine to give uniform flocculent dispersion for GO paper formation, as shown in the schematic (figure 18). C. Huang as well fabricated homogenous GO aqueous suspensions by ultrasonication. It was later freeze dried and obtained as scaffold that was infiltrated by SMP epoxy [125]. GO could also be ultrasonicated in N-Methyl-2-Pyrrolidone (NMP) solvent for preparation of reduced GO which was integrated in SMP epoxy.

Furthermore, mechanical stirring is another approach which can be used to disperse graphene. The approach is largely combined with sonication. For instance, Z. Yu synthesized GO by first mechanically stirring a solution containing graphite powder, $\mathrm{KMnO}_{4}, \mathrm{NaNO}_{3}$ and deionized water. Then, ultrasonication process is followed [126]. 


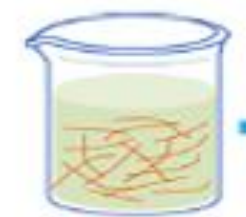

short GO nber
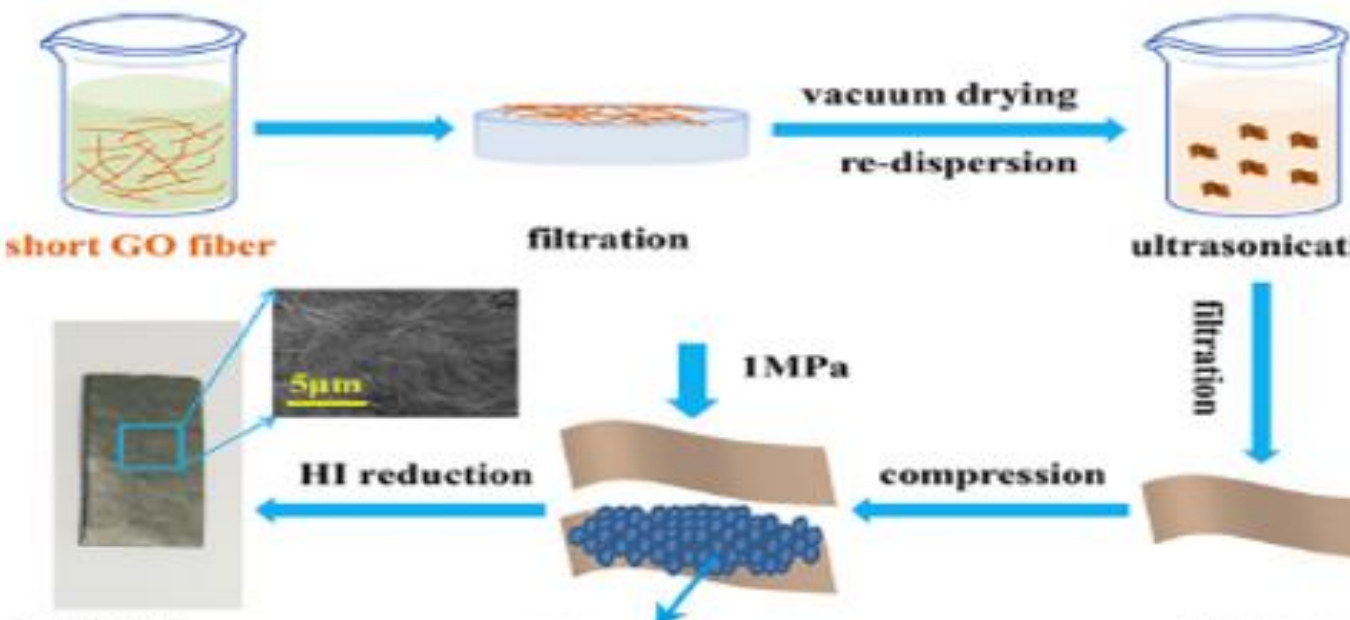

filtration

GO/WEP paper

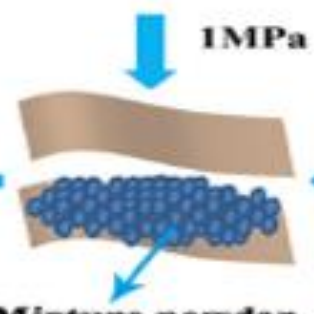

ultrasonication

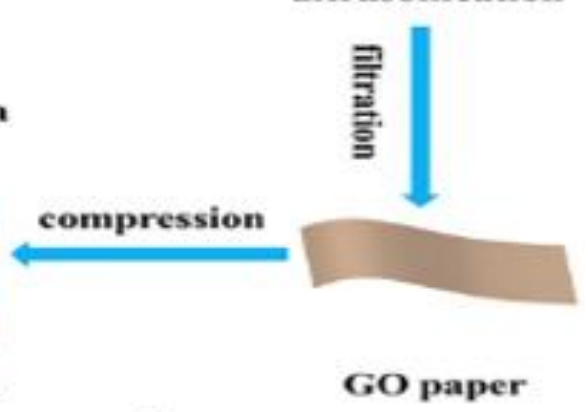

Figure 18: Preparation procedures of RGO/WEP/RGO sandwich structure composite film [124].

\subsubsection{Processing of SMP-graphene Composites}

As excellent dispersion of graphene plays a key role, graphene integration with SMP epoxy is also important. Different techniques used in fabricating SMP epoxy-graphene would be briefly described. Such techniques include resin transfer molding [127], minicalendering [128], vaccum infusion[129], sonication and mechanical stirring[130], [131], and casting [125].

\section{$\underline{\text { Resin transfer molding }}$}

The reinforcing agent (reduced GO paper) was first preloaded into the botton surface of the mold. The polymer mixture containing SMP epoxy resin and hardener was injected into the mold. SMP mixture filled the mold and was left to room-cure [127]. 


\section{Mini-calendering}

Calendering is a manufacturing processing of compressing or smoothing material by passing the material through several heated rolls. A study adopted mini-calendering technique to fabricate SMP epoxy-graphene composite [128]. Three-roll mill was initiaally heated. The SMP-graphene material was passed through it, with step-by-step reduction in the roll gaps. This was performed to achieve uniform distribution of the fillers.

\section{Sonicating and Mechanical Stirring}

SMP epoxy-graphene composites can be fabricated by ultrasonicating. In some instances, mechanical stirring can be added after sonication. A study by E. Wang et al. fabricated SMP epoxy with hybrid filler of GO and CNT by solution mixing [130]. They added CNT/GO aqueous solution to waterborne epoxy and ultrasonicated it for 30 minutes.

This was further freeze dried to give resultant powder and was used as SMP composite film. Y. Wang et al. combined ultrasonication and mechnanical stirring to prepare their composites [131]. Graphene solution was first added to SMP epoxy mixtures by magnetic stirring (200 rpm) and subjected to ultrasonication for 20 minutes. The mixture was further heat to $60^{\circ} \mathrm{C}$ to remove the solvent and stirring was increased to $300 \mathrm{rpm}$. The sample mixture was heated to $80^{\circ} \mathrm{C}$ for $10 \mathrm{hr}$ and poured into the mold for curing. Furthermore, $\mathrm{C}$. Huang et al. fabricated nacre-like SMP epoxy-graphene composites by freeze casting (schematic shown in figure 19) after preparing GO aqueous suspension by ultrasonication [125]. Frozen GO suspension was put in a freeze dryer for 2 days. The resultant GO scaffolds obtained were heated for 1 hour at $800^{\circ} \mathrm{C}$ in nitrogen. It was further filled with SMP epoxy and cured for 2 hours at $130^{\circ} \mathrm{C}$. 


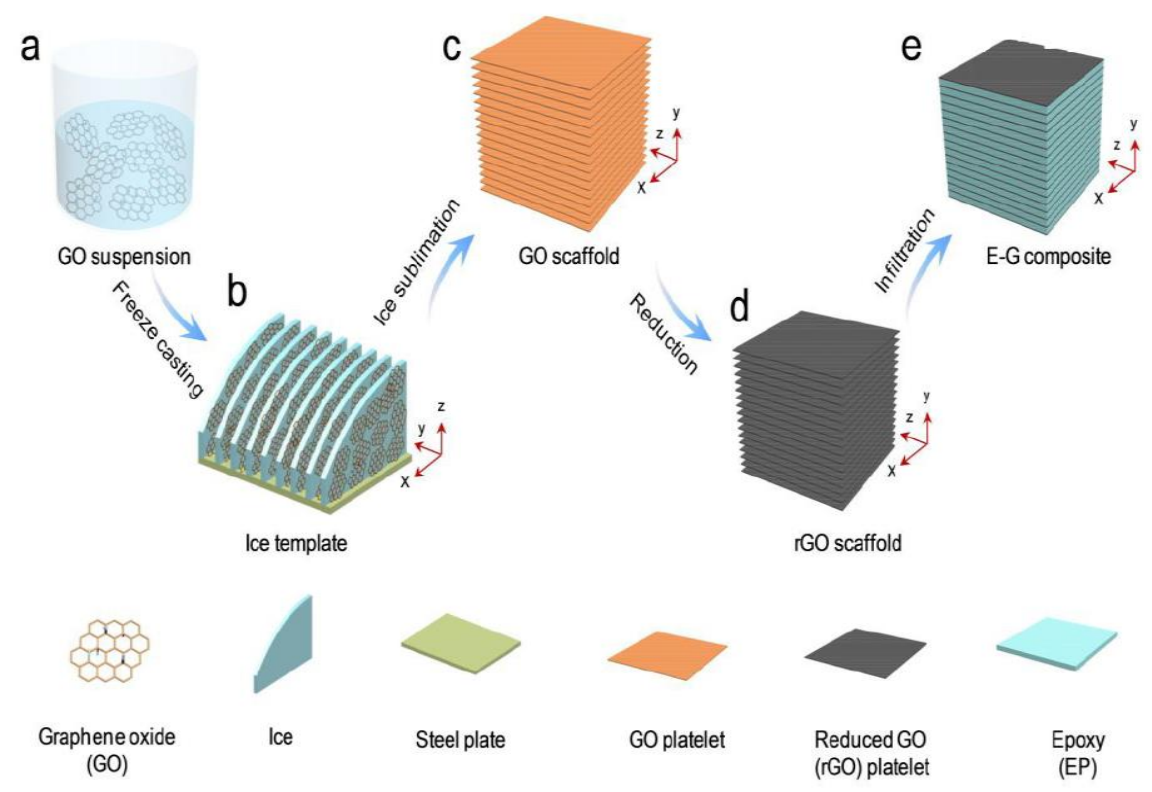

Figure. 19: Schematic of the fabrication of E-G composites. (a) Preparation of GO aqueous suspension with THF, then freezing by bidirectional freeze-casting (b). The lamellar GO scaffold was obtained after freeze drying (c). Subsequently, heating was applied to reduce the GO scaffold, forming an rGO scaffold (d). Finally, the E-G composite was produced by infiltrating with shape memory EP and (e) curing [125].

\section{Casting}

Like casting technique adopted in the fabrication of GrF based polymer technique, SMP-graphene composites can be prepared by the approach. Z. Yu et al. prepared SMP epoxy-graphene mixture at $60^{\circ} \mathrm{C}$ for 3 hours and put in vaccum drying box at $80^{\circ} \mathrm{C}$ for 12 hours [126]. The mixture was finally cast into the mold and cured at room temperature for another 12 hours. Similarly, X. Xiao synthesized SMP-based epoxy composite by casting [132]. They mixed Epon 826, NGDE and Jeffamine D230 together, by hand, in the ratio of 1.3/0.7/1 for 10 seconds. The sample mixture was cast into an aluminum mold, followed by curing at $100^{\circ} \mathrm{C}$ for 1 hour and post-curing at $130^{\circ} \mathrm{C}$ for addition 1 hour.

The processing techniques highlighted indicate robustness in the fabrication of SMP epoxy-graphene composites for smart-based applications. 


\subsubsection{Properties of Graphene-Reinforced Shape Memory Epoxy Composites}

\subsubsection{Shape Recovery Behavior}

Graphene-reinforced SMP epoxy composite is an emerging smart polymer composite. Presently, few studies have investigated graphene influence on shape memory, mechanical and thermal properties of SMP epoxy. Y. Wang et al. examined shape memory performance of graphene-hydro epoxy (H-EP) composite [131]. They revealed that increase in graphene content from 1 to $3 \mathrm{wt} . \%$ resulted in increase in shape recovery ratio from 95.6 to $96.4 \%$. Furthermore, 3 wt.\% graphene addition enhance the recovery speed of SMP epoxy by $25 \%$. Increasing graphene content causes increase in stiffness and themal conductivity, improving the shape recovery ratio and recovery speed. In another study, W. Wang constructed electrically driven SMP epoxy-based composite [127]. They found out that shape recovery speed of RGO-SMP epoxy composites increased as the applied voltage increased. In order words, the recovery speed five times faster at $4 \mathrm{~V}$ than at $6 \mathrm{~V}$. The shape recoverability was nearly $100 \%$ at 6 volts and 5 seconds. It suggests that electrical induction could save more energy than thermal actuation of SMP.

Also, L. Chen et al. incorporated different dimensions of nanocarbon (carbon black, CNT, graphene and functionalized graphene sheet (FGS)) in microwave responsive SMP epoxy [128]. FGS reinforced SMP epoxy exhibited shortest recovery time (250 seconds) compared to that of carbon black-based composite (600 seconds). The quick heat diffusion could be attributed to large BET surface area of FGS $\left(385.7 \mathrm{~m}^{2} / \mathrm{g}\right)$. Strong interface adhesion between the FGS and SMP epoxy could also minimize the heat diffusion resistance. Further, X. Xiao et al evaluated recovery or self-healing of SMP epoxy-based composites by performing scratch test using Vickers indenter [132]. Addition of nanolayer 
graphene enhances the SMP scratch resistance. Qualitatively, neat SMP epoxy showed large cracks along the crack direction. Meanwhile, under similar scratching conditions, only small cracks were observed in the first SMP epoxy-graphene sample and no visible cracks were seen on the second SMP epoxy-graphene sample (figure 20). It suggests that improvement improvement in scratch resistance is ascribed to the remarkable in-plane fracture strength of the graphene sheets. Weak interlayer interactions between the nanolayers could also engender easy interlayer movement. This enables energy absorption, preventing further crack formation or propagation.

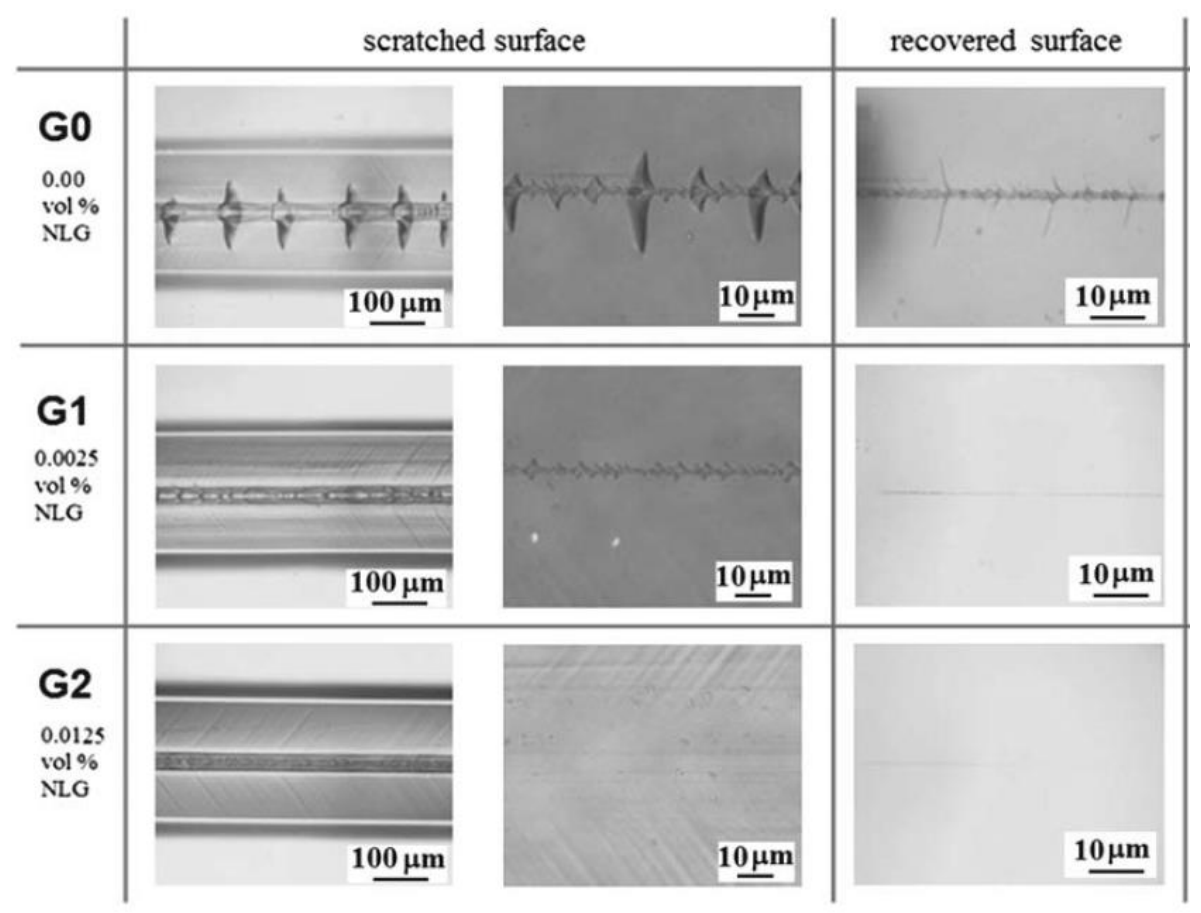

Figure 20: Surface images of the as scratched and recovered surfaces. The optical images in the left two columns represent samples after scratch testing. The optical images in the third column are the recovered samples after heating [132]. 


\subsubsection{Uniaxial and Dynamic Mechanical properties}

Effect of graphene on mechanical and thermal behavior SMP epoxy points to the possibility in performance enhancement of SMP epoxy composites. To illustrate, Z. Yu et al. investigated the uniaxial properties of SMP epoxy-graphene composites [126]. The tensile fracture stress of the composites was approximately $35 \%$ greater than the SMP epoxy resin (figure 21a). This can be attributed to good interface, resulting in more interaction between the filler and matrix. Improvement in dynamic mechanical analysis (DMA) properties was also noticed after GO addition. Storage modulus of the SMP epoxy composite increased at 0.4 and 0.8 wt. $\%$ graphene by roughly $35 \%$ at $75^{\circ} \mathrm{C}$ than that of SMP epoxy (shown in figure 21b). This could be due to increase stiffness from the graphene and interfacial stress transfer from the SMP epoxy to the graphene.

(a)

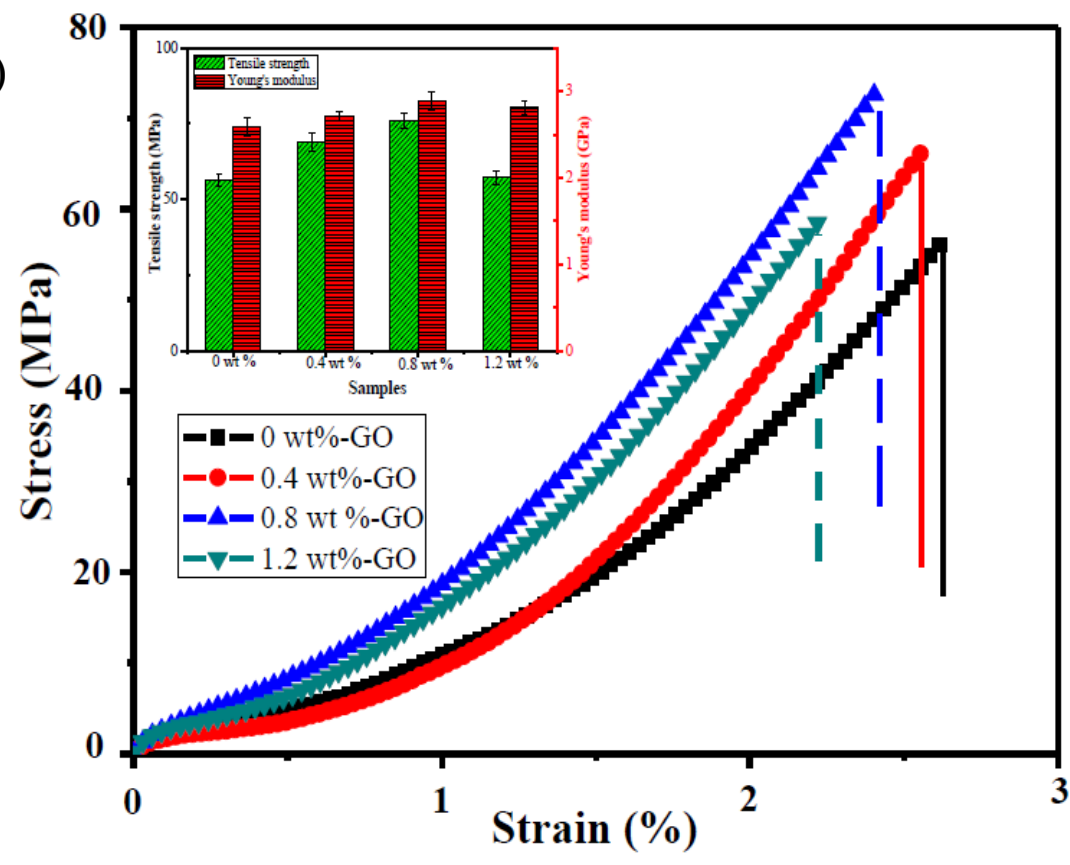




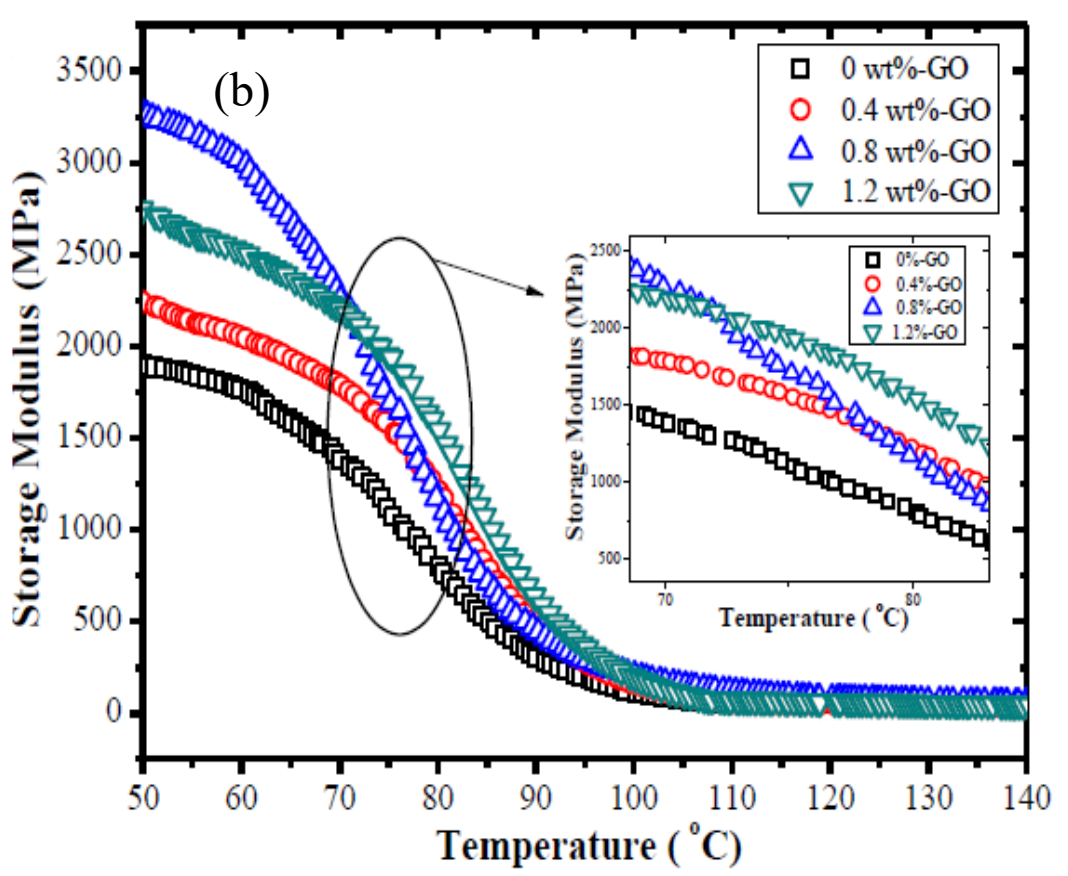

Figure 21: (a) Stress-strain curves (b) DMA curves of storage of GO-toughened epoxy resin composites [126].

Similarly, another study from X. Liu et al. probed the DMA properties of electroactive SMP epoxy-graphene/CNT composite [129]. Below glass transition temperature (Tg), the storage modulus of the composite is greater than that of the epoxy matrix. Restriction of the matrix chains by graphene and CNT could account for increase in composite's storage modulus. However, low storage modulus of the composite above the Tg could be due to weakened cross-linking density. Additional properties such thermal conductivity was also considered. E. Wang examined the thermal conductivity of SMP epoxy-GO/CNT composite [130]. Compare to SMP epoxy, thermal conductivity coefficients of SMP epoxy-GO and SMP epoxy-GO/CNT composite are180\% and 375\% higher, respectively. These remarkable phenomena could be justified from the physical properties of the hybrid fillers. Synergistic effect between GO and CNT could give these significant conductivity 
increase. Also, combination of GO and CNT could form a 3D conductive network framework. The framework creates large surface area between the fillers and the epoxy. This further creates more available pathway for phonon diffusion and and limit thermal interfacial resistance.

\subsubsection{Potential Applications of Graphene-Based Polymer and SMP Composites}

The excellent properties of graphene reinforcement and SMP matrix system make the composite an eye-catching candidate for science and industrial-based communities. Graphene-based polymer and SMP composites can fit into different applications, ranging from aerospace and automobile to biomedical and electronics [119]. Electronic industry can use the emerging graphene based SMP composites in flexible electronics, electrochemical biosensors, super-capacitors, strain sensors [133], [134] etc. The sensivity, flexibility and stability of graphene reinforced SMP composites bring up its usefulness in structural health monitoring [135]. Similarly, the stretching and relaxation movements of SMP epoxy-based composites can help in motion detection of the human body, serving as wearable biosensors [136]. Some of these SMP composites can also find its use as electrochemical sensing such as in detection of bases and enzymes [137]. Graphene based SMP composites could provide high-performance super-capacitors with the improved cyclic stability [138]. Furthermore, for its thermal applications, graphene based SMP composites demonstrated high potentials in thermal management of microelectronics devices. Thermal properties of graphene-SMP composites offer enough capacity for the composites to serve as thermal interface materials (TIM) for semiconductor chips, supplying low thermal resistance and high thermal conductivity [139]. 
It is important to note, again, that despite the improvements in the shape memory behavior of 2D graphene reinforced epoxy SMPs, the challenges such as weak interfacial interaction and graphene sheet aggregation still remain. Report from Wang et al. also highlighted that graphene restacking can result in lower epoxy SMP storage modulus to produce low shape recovery force of the epoxy SMP [131]. Hence, the need to incorporate 3D graphene foam reinforcement in SMP epoxy to completely harness graphene properties. 


\section{Chapter III: Materials and Methods}

This chapter presents materials and methods used in synthesizing GrF reinforced SMP epoxy composites. The characterization techniques and experimental setup employed to obtain information on the microstructure, mechanical, thermal, and shape memory properties are also described.

\subsection{Materials}

Two forms of epoxy resins and a diamine component were used as SMP matrix in this study. The two epoxies are diglycidyl ether of bisphenol A (DGEBA) and neopentyl glycol diglycidyl ether (NGDE). The diamine component is poly(propylene glycol)bis(2aminopropyl) ether. Graphene Foam was used as a reinforcement or filler component to primarily improve shape recovery behavior of the SMP matrix. In the subsequent subsections, the properties of these materials would be provided.

\subsubsection{SMP Epoxy Components}

\subsubsection{Diglycidyl ether of bisphenol A}

Diglycidyl ether of bisphenol A (DGEBA) is a commercial epoxy resin, synthesized by Hexion Specialty Chemicals (Columbus, OH, USA). It is produced by reacting bisphenol A with epichlorohydrin. It has a trademark name known as EPON 826. DGEBA is aromatic. It is a colorless liquid, with a density of $1.16 \mathrm{~g} / \mathrm{ml}$. DGEBA has low viscosity of $0.64-0.95 \mathrm{cP}$ at $25^{\circ} \mathrm{C}$, with a molecular weight of $340 \mathrm{~g} \mathrm{~mol}^{-1}$. 


\subsubsection{Neopentyl glycol diglycidyl ether}

Neopentyl glycol diglycidyl ether (NGDE) is an ether epoxide derivative. It is obtained from Sigma Aldrich (St. Louis, Missouri, USA). It is a long-chain epoxide, having two epoxide groups at the end of the chain. NGDE is aliphatic and is colorless liquid. It also has a low viscosity of $10-30 \mathrm{cP}$ at $25^{\circ} \mathrm{C}$. Its density and molecular weights are 1.04 $\mathrm{g} / \mathrm{ml}$ and $216.28 \mathrm{~g} \mathrm{~mol}^{-1}$, respectively.

\subsubsection{Poly(propylene glycol)bis(2-aminopropyl) ether}

Poly(propylene glycol)bis(2-aminopropyl) ether belongs to the family of polyamines. It synthesized by Huntsman Corporation (Woodlands, Texas, USA). Its trademark name is known as Jeffamine D230. The backbone chain of Jeffamine D230 consists of repeated oxypropylene units. It is a difunctional, primary amine and has an average molecular weight of approximately 230 . The primary amine groups are situated on secondary carbon atoms at the end of the aliphatic polyether chain. Like EPON 826 and NGDE, Jeffamine D230 is colorless liquid and has an ammoniacal odor. It's relative density and dynamic viscosity at $20^{\circ} \mathrm{C}$ are 0.9476 and $10.3 \mathrm{cP}$.

\subsubsection{D Graphene Foam}

The GrF selected for reinforcement of the shape memory epoxy matrix is procured from Graphene Supermarket (Calverton, NY, USA). Figure 22a shows an SEM image of the hierarchical structure of the as-received GrF. The manufacturer from Graphene Supermarket synthesized graphene foam using the template-directed CVD approach, which was described in the literature review. Free standing graphene foam has a pore size of $580 \mu \mathrm{m}$ and 1-2 mm thickness. Due to its approximately $99.7 \%$ high porosity, graphene 
foam has an ultra-low density of $4 \mathrm{mg} / \mathrm{cm}^{3}$. Its hollow branch diameter is $50 \mu \mathrm{m}$. The foam architecture consists of 5-7 thin interconnected layers of graphene sheets.

\subsubsection{Graphene Nanoplatelets}

Graphene Nanoplatelets (xGNP-M-5) were procured from XG Sciences, Lansing, MI, USA. It has a thickness of 6-8 $\mathrm{nm}$ [140]. This implies that an average GNP particle consist of about 20 graphene sheets as an individual pair of graphene layers has $\sim 0.35 \mathrm{~nm}$

thickness [141]. Having an average diameter of $15 \mu \mathrm{m}$, GNP particles exhibits a relative surface area of $120-150 \mathrm{~m}^{2} / \mathrm{g}$ [140]. GNPs contains some functional groups at its edges, consisting of approximately $0.03 \%$ hydroxyl groups, $0.035 \%$ carboxyl and $0.075 \%$ ether [140]. Figure 22b shows the SEM image of wrinkled and folded GNPs.

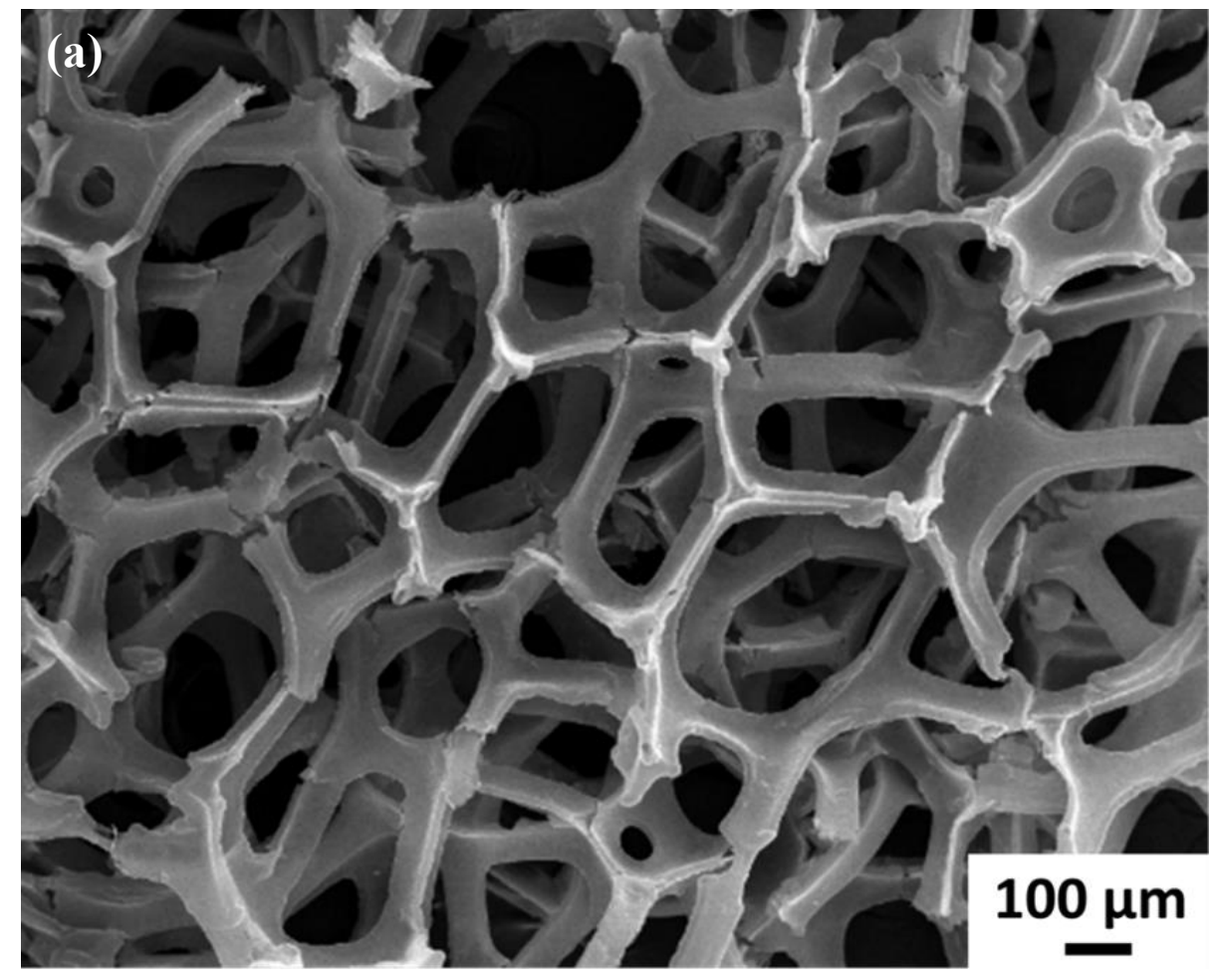




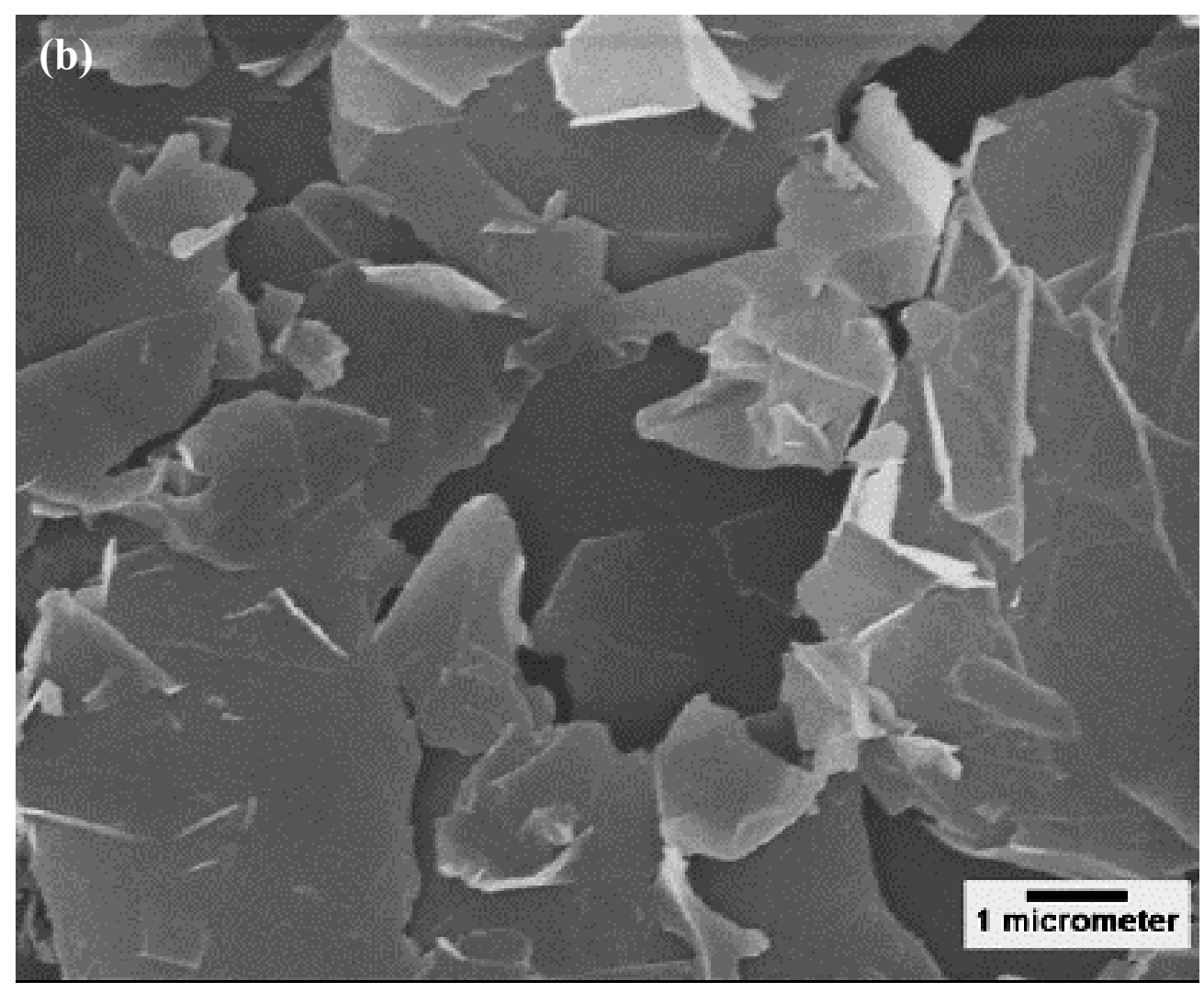

Figure 22: SEM micrograph of (a) as-received GrF made by chemical vapor deposition (CVD) [78] (b) SEM image of as-received graphene nanoplatelets (xGNP-M-5) were obtained from XG Sciences [158]

\subsection{Experimental Procedure}

\subsubsection{Preparation of SMP Epoxy}

SMP epoxy consists of a mixture of three components: Diglycidyl ether of bisphenol A (or EPON 826), neopentyl glycol diglycidyl ether and poly(propylene glycol)bis(2aminopropyl) ether. Volume ratio for SMP epoxy formulation from the three components was selected as 1:1:1. The selection was preferred because it would give optimum good 
shape memory behavior for SMP epoxy [118]. Subsequently, EPON 826 was first weighed $\left(2.98 \mathrm{ml}\right.$ ) into a ceramic cup and melted by heating in an oven preset at $70^{\circ} \mathrm{C}$ for $15 \mathrm{~min}$. This was followed by pouring the melted EPON 826 along with the weighed Jeffamine D230 and NGDE into a cylindrical plastic container. The three components were stirred and shaken vigorously by hand for about 10 s to mix them thoroughly. The volume of each component was obtained by determining the reacting mass from the ratio of number moles and respective molecular weights; then the volume was known from the ratio of their reacting mass to respective densities.

\subsubsection{Mold Casting of SMP Epoxy-GrF Composite}

\subsubsection{Fabrication of Rectangular Shape Samples}

Aluminum pan was used as the mold to fabricate rectangular samples of the composite. Free standing $\mathrm{GrF}$ of dimensions of $40 \mathrm{~mm}$ in length, $2 \mathrm{~mm}$ in width and 1.2 $\mathrm{mm}$ in thickness was in-situ inside the pan. The mixed liquid resin was poured into the pan at room temperature, infiltrating GrF till it is fully covered with SMP epoxy. The samples were placed in the oven for it to polymerize. For it to polymerize, samples were thermally cured in the oven at $100^{\circ} \mathrm{C}$ for 1.5 hours and post-cured at $130^{\circ} \mathrm{C}$ for 1 hour. After complete polymerization, the samples were demolded and cut into rectangular shapes $(1 \times 3 \times 50$ $\mathrm{mm}$ ) for shape recovery and DMA characterization. The same fabrication process was performed for the neat SMP epoxy samples. A schematic to describe the fabrication process is shown in Figure 23. 


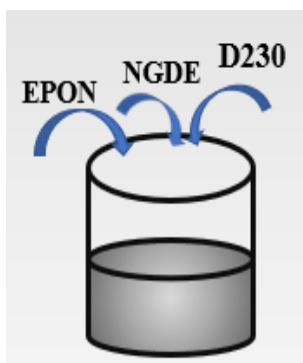

Pour the polymer mixtures at room temperature

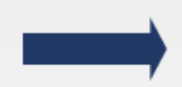

Stir the polymer mixtures at room temperature for 15 secs
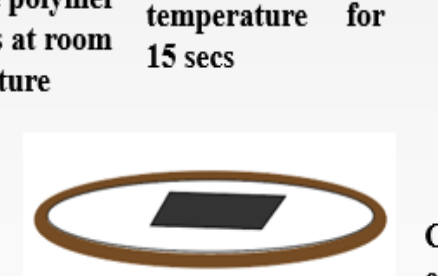

0.75 wt. $\%$ GrF-epoxy composite

r

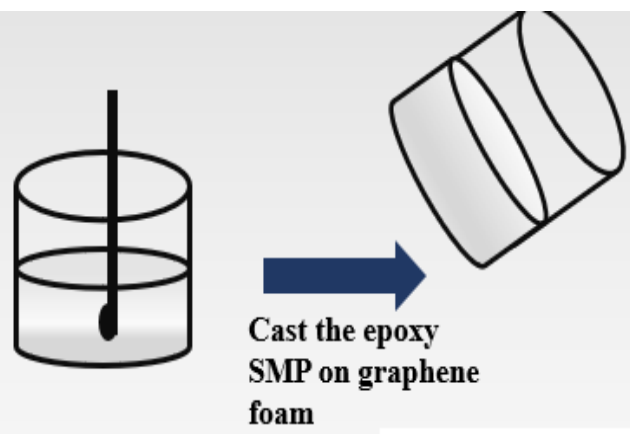

Free-flow infiltration of GrF with epoxy SMP

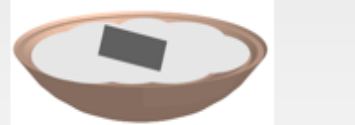

Place the GrF-epoxy composite in the oven

Figure 23: Schematic of a Mold Cast Thermal Responsive SMP-GrF Composite

\subsubsection{Fabrication of Dog-Bone Shape Samples}

SolidWorks CAD software was utilized to design a reverse impression dog-bone mold fixture. The reverse impression fixture was manufactured using aluminum material, as shown in Figure 24. The aluminum mold is used to create a silicone dog-bone mold (which was employed to produce dog-bone SMP epoxy composites). The silicone mold was coated with mold release agent and allowed to air dry. GrF were cut into three-strip pieces having dimension $30 \times 4 \mathrm{~mm}$. Each piece was weighed individually. A syringe was used to dispense a layer of SMP epoxy into the bottom of the casting silicone mold. The GrF strips were placed over the epoxy into the gauge length of the mold (figure 25). The samples were placed in the oven at $100^{\circ} \mathrm{C}$ and cured for 1.5 hours and post cured at $130^{\circ} \mathrm{C}$ for 1 hour. The same procedure was adopted to fabricate the control samples. All the samples were demolded after complete curing for uniaxial tensile testing. 


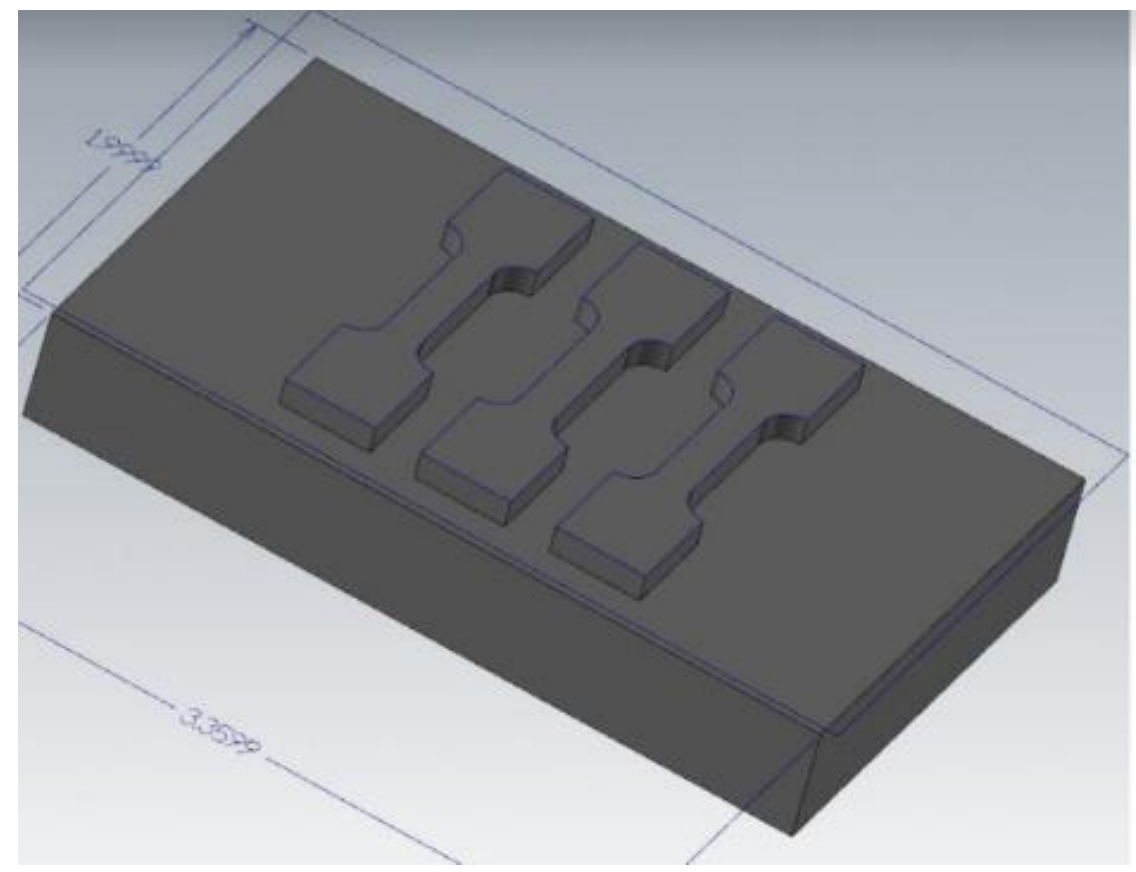

Figure 24: Reverse impression dog-bone mold fixture

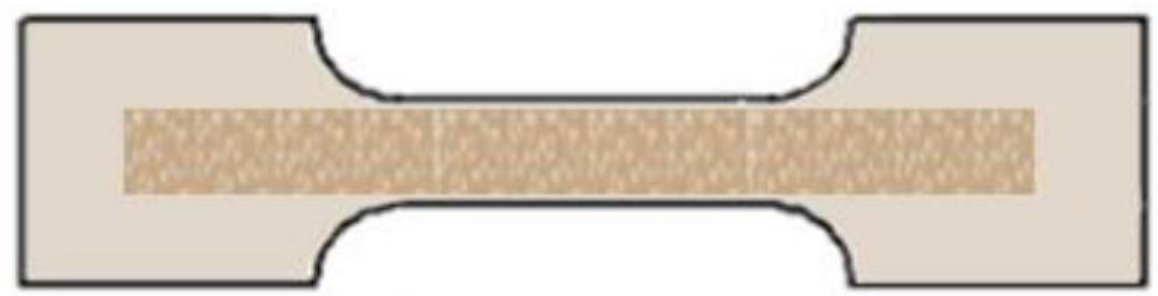

Figure 25: Schematic of SMP epoxy-GrF mold cast Composite

\subsubsection{Fabrication of Electrically Responsive SMP Epoxy-GrF Composites}

Same dimensions of GrF used in section 3.2.2.1 was connected to a platinum wire (Pt) of $0.1 \mathrm{~mm}$ diameter (Surepure Chemetals, LLC, Florham Park, NJ) using a conductive Pelco colloidal silver paste (Clovis, CA). The schematic is shown in figure 26. Addition of the silver paste between the interface of the Pt wire and $\mathrm{GrF}$ allows contact resistance to be 
minimized during current-induced heating of $\mathrm{GrF}$. The silver paste was cured at $100^{\circ} \mathrm{C}$ for 30 min. Premixed SMP epoxy was cast, infiltrating the graphene foam-wire connection laid in flat pan aluminum mold. The new configuration of SMP epoxy-GrF composite sample was similarly cured and post-cured at $100^{\circ} \mathrm{C}$ for 1.5 hours and $130^{\circ} \mathrm{C}$ for one $\mathrm{h}$, respectively.

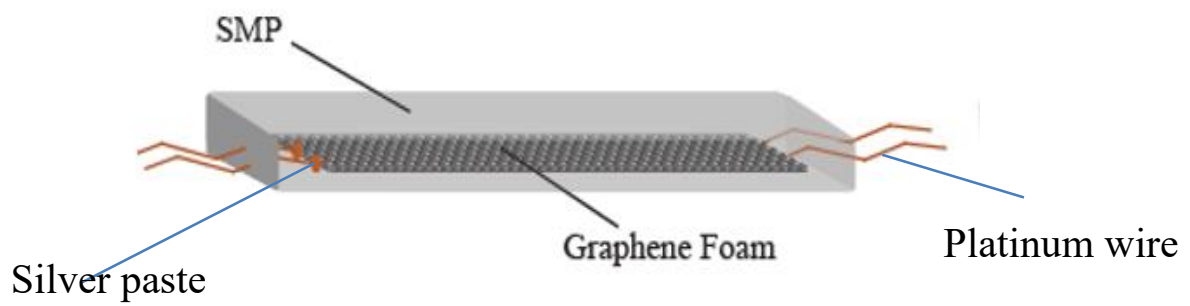

\footnotetext{
Graphene foam as a conductor embedded inside non-conducting polymer.

Graphene foam-epoxy SMP composite to be subjected to joule heating.
}

Figure 26: Schematic of a fabricated Electrically Responsive SMP-GrF Composite

\subsubsection{D Printing of SMP Epoxy and Graphene-Based SMP Epoxy Composites}

3D printing of dog-bone shape of SMP epoxy and its graphene-based counterpart was performed using a Hyrel System 30M printer with syringe dispensing system (SDS) extruder. Settings considered for the SDS extruder include software for the stepper motor, nozzle diameter and "start/end" G-code. The 3D dog-bone models for printing were designed using SolidWorks CAD software. All STL files obtained from SolidWorks were processed by an open source free software Slic3r (http://slic3r.org/), an open-source 3D printing toolbox. Slic3r sliced the files into $200 \mu \mathrm{m}$ thick layers to generate G-code 
instructions for the 3D printer. The G-code was created using the spiral option in the Slic3r software. The G-code was sent to the printer using Repetrel software, a proprietary 3D printer host software suite designed by Hyrel 3D company.

Before 3D printing, a glass slide was placed on the printer bed. The glass slide serves as the plate where the material would be directly printed. Also, the viscosity of SMP epoxy resin and the epoxy-graphene solution was determined using a viscometer. SMP Epoxy has a viscosity $730 \mathrm{cP}$, and it was drawn into a $60 \mathrm{~mL}$ plastic syringe. It was then capped with an $800 \mu \mathrm{m}$-ID 0.75" stainless steel deposition tip (Nordson EFD). The syringe was mounted into the SDS extruder of the 3D printer. Extruder nozzle was positioned at the bottom center of the printer bed, and the Z-position of the printer bed was set to zero position for the printing of the dog-bone shape samples. Printing occurred at a typical speed of $20 \mathrm{~mm} / \mathrm{s}$, taking 2.5 minutes to print each sample. During printing, freeze spray aerosol was sprayed on the extruded material. This was done to prevent material smudging because SMP epoxy is temperature-sensitive. After printing, the glass slide containing the printed samples were placed in the refrigerator. The printed samples were subjected to a low temperature of $10^{\circ} \mathrm{C}$ for 24 hours to prevent further any smudging that could occur, helping the material to retain the dog-bone shape configuration. The SMP epoxy-based samples were then allowed to cure for an additional 72 hours at room temperature. The rigid prints were demolded and gently removed from the glass slide at $40^{\circ} \mathrm{C}$. 


\subsection{Microstructural Characterization}

\subsubsection{Scanning Electron Microscopy: Powder Morphology and Microstructure of Composites}

JEOL JSM-633OF field emission scanning electron microscope (FE-SEM) operating at $15 \mathrm{kV}$ was used for the characterization of SMP epoxy powder, cured SMP epoxy and SMP epoxy-GrF composites. The primary use of FE-SEM was in investigating the fracture surface of tensile specimens made from casting and 3D printing techniques. SEM deep depth of field and large depth of focus at low magnifications enable it to be a robust technique to extract information from smooth and rough fracture surfaces on the failure mechanisms of SMP epoxy-based samples. Fracture surfaces were prepared by subjecting the samples under a tensile load till breakage point and examined the broken surfaces within the gauge length region of the samples. Samples were sputter-coated with gold for $50 \mathrm{sec}$ before examining in SEM to avoid artifact due to charging. Fracture surfaces of the cast and 3D printed samples were observed under SEM to analyze the infiltration of GrF its bonding with SMP matrix and graphene dispersion in the matrix, respectively. Also, the low magnification top view of the samples was shown with a digital microscope (DinoLite, Dunwell Tech, Inc., Torrance, CA, USA) during tensile testing.

\subsubsection{Raman Spectroscopy}

Raman spectroscopy is a very important surface characterization technique for probing molecular vibrations. They are based on the interaction of electromagnetic radiation with vibrating modes of a molecule. Raman spectroscopy is specifically based on inelastic scattering of monochromatic incident radiation when the radiation interacts with the vibrating molecules vibrational modes of a molecule [142]. Raman spectroscopy was 
conducted to show characteristics plot for all specific type of bonds present in the samples. This could be construed as the presence of a chemical compound. Raman spectroscopy on SMP epoxy-based specimens was performed using a Spectra-Physics (Model 3900S, CA) equipped with Ti sapphire crystal $(514 \mathrm{~nm})$ as the target. The Spectra-Physics also consists of a detector with $4 \mathrm{~cm}-1$ spectral resolution from Kaiser Optical Systems, Inc. (Michigan, USA), a laser power (18 $\mathrm{mW})$ and spot size of $6 \mu \mathrm{m}$.

\subsubsection{Fourier Transform Infrared (FTIR) Spectroscopy}

FTIR is also an essential technique to reveal surface functionalities of a material. It is the study of the interaction between electromagnetic fields and matter within the infrared region, showing the properties of the matter. The interaction of IR radiation with a molecule can make the molecule to absorb radiation and excite to a higher vibration state. In order words, if the energy of the photon coincides with the molecule's vibrational energy of the molecule, a frequency will be strongly absorbed. It gives fingerprint information on the chemical composition of the sample. FTIR spectra of SMP epoxy-based composite samples were recorded using FT/IR 4000 type spectrophotometer (JASCO FT/IR, 4100, Tokyo, Japan) within a range from 4000 to $400 \mathrm{~cm}^{-1}$.

\subsection{Thermal Characterization}

\subsubsection{Differential Scanning Calorimetry (DSC)}

DSC is one of the most used thermal analysis techniques to evaluate heat flows that are involved in a material transition as a function of temperature and time. It provides qualitative and quantitative information about chemical and physical changes during a change in heat capacity of the material. The changes point to glass transition temperature 
(Tg) for materials such as thermoset polymers. DSC measurements of SMP epoxy and SMP epoxy-GrF composite were performed using SDT Q600 from TA Instruments (New Castle, DE, USA). $8 \mathrm{mg}$ of sample was enclosed in an aluminum pan and subjected to heating rate of $10^{\circ} \mathrm{C} / \mathrm{min}$ from room temperature to $175^{\circ} \mathrm{C}$. It was under an argon atmosphere and a purge gas flow rate of $50 \mathrm{~mL} \mathrm{~min}^{-1}$. The inflection temperatures obtained in the DSC thermogram curves are the glass transition temperatures of SMP epoxy and SMP epoxy-GrF composites.

\subsubsection{Thermal Conductivity}

A flash thermal conductivity technique (LFA 467, NETZSCH, Germany) was employed for evaluation of the thermal diffusivity $\left(\alpha, \mathrm{mm}^{2} / \mathrm{s}\right)$ and the size of each measured circular sample at $25^{\circ} \mathrm{C}$ is $12.7 \mathrm{~mm}$ in diameter, $0.792 \mathrm{~mm}$ in thickness (for SMP epoxy) and $1.09 \mathrm{~mm}$ in thickness (SMP epoxy-graphene). The thermal conductivity $(\lambda, \mathrm{W} / \mathrm{mK})$ of SMP epoxy composites and SMP epoxy was then calculated as;

$$
\lambda=\alpha \times \rho \times \mathrm{Cp}
$$

$\alpha$ is the thermal diffusivity, $\rho$ is the density and $\mathrm{Cp}$ is the specific heat capacity Thermal diffusivity of the samples within the temperature range of 25 to $70^{\circ} \mathrm{C}$ was obtained, and its thermal conductivity was determined at $25^{\circ} \mathrm{C}$ and $70^{\circ} \mathrm{C}$.

\subsubsection{Dilatometry}

Dilatometry is a widely adopted technique for the determination of the coefficient of linear thermal expansion of specimens, particularly polymeric materials. In this approach, the sample is placed inside a dilatometer consisting of a furnace, push rod and high resolution linear variable differential transformer (LVDT) (as a sensor unit). The core of 
LVDT is joined to the sample through the pushrod and move-in coordination to specimen expansion during heating. Then after heating, thermal expansion is obtained. Dilatometry measurement of SMP epoxy samples was performed with the aid of DIL 802/802L differential horizontal dilatometer under air at $10^{\circ} \mathrm{C} / \mathrm{min}$ from room temperature to $40^{\circ} \mathrm{C}$. Dimensional change and coefficient of linear thermal expansion of the samples were obtained.

\subsection{Shape Memory Characterization}

\subsubsection{Thermally Stimulated SMP Epoxy-GrF Composite}

Rectangular samples of SMP epoxy-GrF composite having dimensions 50 × 3 × 1 $\mathrm{mm}$ was prepared, as highlighted in section 3.2.2.1. The sample was placed on a hot plate at $70^{\circ} \mathrm{C}$ for $5 \mathrm{~s}$. Then, it was manually deformed into a temporary ' $\mathrm{U}$ ' shape with a radius of $3 \mathrm{~mm}$ in its rubbery state, after it was immediately removed from the hot plate. Promptly, the sample was dipped in the sample in a cold-water bath $\left(20^{\circ} \mathrm{C}\right)$ for $6 \mathrm{~s}$ while the external constraint was maintained. This is done to lock the elastic deformation energy and retain the deformed shape. The deformed ' $\mathrm{U}$ ' shape sample was returned to the hot plate at $70^{\circ} \mathrm{C}$ for $5 \mathrm{~s}$, recovering to its original rectangular shape.

\subsubsection{Qualitative and Quantitative Evaluation of Shape Recovery of SMP Epoxy- GrF Composite \\ Qualitative evaluation of shape recovery of the composite samples was performed} with the aid of a Nikon D3500 DSLR camera. The camera was used to record videos and take images of how the deformed shape returns to its original shape. Shape recovery time was obtained from the videos. Image $\mathbf{J}$ was employed to quantify the shape recovery of the samples. Image $\mathbf{J}$ is an open-source Java-based image processing program tailored for 
scientific dimensional images. Shape recovery angles of the samples concerning time were measured with the Image $\mathbf{J}$ software, and shape recovery ratio and the rate were calculated from the recovery angle and time.

\subsubsection{Electrically Stimulated SMP Epoxy-GrF Composite}

Electrical actuation based on current-induced heating was applied to the bent predeformed GrF-based SMP epoxy samples described in section 3.2.2.3 to recover to its original flat shape. The current-induced heating was performed by supplying a direct current through the composite. Current was produced by a four-point probe technique, using a Keithley 2401 digital source meter (Cleveland, OH, USA). Two pairs of probes, connected to the DC source for current supply, were attached to the platinum wire electrodes of $0.1 \mathrm{~mm}$ diameter (Surepure Chemetals, LLC, Florham Park, NJ) in contact with the SMP epoxy-GrF composite. Flow of electric current through the GrF embedded in the SMP epoxy matrix induces heat generated to the temperature of $70^{\circ} \mathrm{C}$. The heat is enough to restore the bent shape to original flat shape. Similar approach using the camera and Image $\mathbf{J}$ is used to quanitify the recovery of the electrical responsive SMP epoxy-GrF composite.

\subsubsection{Thermal Imaging of Electrically Triggered SMP Epoxy-GrF Composite}

Thermal imaging was performed when current-induced heating propagates through the composite. This was conducted by using FLIR T620 High-Resolution Infrared Thermal Imaging camera (Wilsonville, OR). The camera served two functions concomitantly. It observed the surface temperature changes of the samples. It also recorded and revealed heat transport or distribution through the GrF to the entire SMP epoxy matrix. 


\subsection{Evaluation of Mechanical Behavior}

\subsubsection{Uniaxial Tensile Test}

Tensile strength of cast and 3D printed samples was determined by conducting uniaxial tensile testing using a mechanical testing stage (SEMtester 1000, MTI Instruments, Inc., Albany, NY, USA), shown in figure 27. The mechanical testing stage load cells (4400 and $440 \mathrm{~N}$ ) were used to perform tensile testing for casting, and 3D printed samples, respectively. The stage is controlled using MTEST Quattro software (ADMET, Norwood, MA, USA). The tests were performed by holding the sample between the grip fixtures and applying a force that stretched the sample gauge length to a failure at a rate of $1 \mathrm{~mm} / \mathrm{min}$. Load-displacement data and plot is obtained from the software after the test is completed.

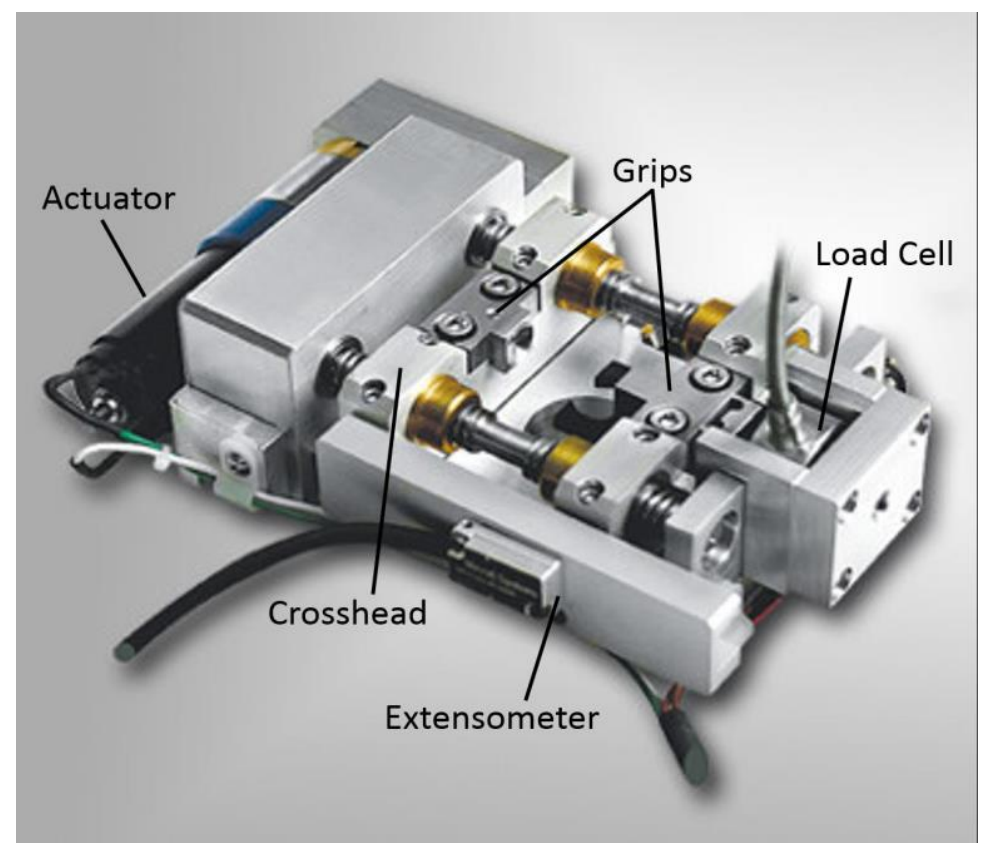

Figure 27: Image showing mechanical MTI testing stage used for tensile testing [162]

To determine the accurate representation of the material property, the loaddisplacement result is normalized. Load data is normalized with a cross-sectional area of 
the sample while displacement is normalized with the sample's gauge length. The standardization of data gives the stress-strain result of the sample, as indicated in the following equations:

$$
\sigma=\frac{F}{A}
$$

where $\sigma=$ tensile stress; $F=$ force applied and $A=$ cross-sectional area

$$
\varepsilon=\frac{\Delta L}{L}
$$

where $\varepsilon=$ tensile stress; $\Delta L=$ force applied and $L=$ original length

This means that stress is obtained by the basic calculation of dividing the applied force by sample cross-sectional area. The strain calculations are based on strain analysis using DLTdv packages in MATLAB platform. DLTdv package is a direct linear transformation for digitizing (2D motion) videos containing strain data. After digitizing, the package provides information on $\frac{\Delta L}{L}$, which is plotted against stress to give a stress-strain plot of the SMP epoxy-based samples.

\subsubsection{Dynamic Mechanical Analysis (DMA)}

Dynamic mechanical performance was conducted using Electroforce 3100 from TA Instruments (New Castle, DE, USA) and DMA 242 Artemis from NETZSCH Instruments (Burlington, MA, USA) in tensile, bending and multifrequency modes. The Electroforce is run by WinTest software, which contains the DMA application, and interfaces with TRIOS software. An external MTI heater unit (SEMtester 1000, MTI Instruments, Inc., Albany, NY, USA) was added and positioned beneath the tensile fixture of the Electroforce 
3100 instrument. This was done because the instrument does not have an in-built furnace that can subject the material above room temperature.

Rectangular samples of SMP epoxy-GrF with dimensions of $10 \mathrm{~mm}$ long, $8 \mathrm{~mm}$ wide and $1.38 \mathrm{~mm}$ thick were used. Using the Electroforce with a force transducer of 1000 gram, the samples were placed within the tensile grip fixtures. As the sample is subjected under displacement control, an amplitude load range (50-5 $\mu \mathrm{m})$ and a frequency sweep $(10-100$ $\mathrm{Hz}$ ) was applied on it. This was carried at different temperatures (from $25-70^{\circ} \mathrm{C}$ ). The temperature range was based on the environmental temperature for some structural applications. The TRIOS software provides the output results containing the tan delta of the samples.

SMP-epoxy-GrF sample was further subjected to bending deformation using DMA 242 Artemis. The sample was placed in a 3-point bending deformation fixture and enclosed in a furnace. The control type selected is mixed control. A constant static force is not applied to the sample as the force would change as the sample greatly softens during the glass transition. A static preload is therefore set by setting the proportional factor (PF) of 1.1. Thus, static preload can be determined as;

$$
\mathrm{F}_{\text {Static preload }}=P F \times F_{\text {Dynamic }}
$$

A dynamic load of $2 \mathrm{~N}$ and specific amplitudes of 50, 100, and $200 \mu \mathrm{m}$ are applied within the temperature range from $25-100^{\circ} \mathrm{C}$ at heating rate of $3 \mathrm{~K} / \mathrm{min}$ and $1 \mathrm{~Hz}$. After test completion, the storage and loss moduli and tan delta curves are obtained using the DMA 242 software. 


\section{Chapter IV: Results and Discussion}

The overall goal of this research is to enhance the shape recovery ability of GrFreinforced shape memory epoxy composites, which can be used to make low density, flexible, and morphing structures. To accomplish the objective, structure-processingproperty of the composite is significantly considered. Micro-structural characteristics of the composite are elucidated to portray good interfacial interaction between $\mathrm{GrF}$ reinforcement and SMP epoxy matrix. Shape recovery behavior is evaluated to confirm enhancement in recovery performance of the GrF-based composites fabricated by a cast and 3D printed samples. Thermal studies are undertaken for understanding GrF influence in $\mathrm{Tg}$ and expansion behavior of SMP epoxy. Mechanical performance of the reinforcement in the matrix is pursued to explain the role played by fundamental mechanisms in the matrix property enhancement. These findings are thoroughly explained in the following sections.

\subsection{Morphology of SMP Epoxy-GrF Composites prepared by Mold Casting}

$\mathrm{GrF}$, which is employed to reinforce SMP epoxy, is a macro-porous framework of interconnected graphene sheets. Figure 22 shows the hierarchical structure of the asreceived GrF exhibiting branch-node architecture. Integration of SMP epoxy-GrF composite requires that SMP epoxy permeates through the interconnected nano-pores and wets the branches and nodes of GrF. Its infiltration characteristics control permeation of SMP epoxy. This is further contingent on SMP epoxy viscosity and GrF pore size. SMP epoxy in this study has low viscosity $(25.6 \mathrm{cP})$, which easily percolates through the $\mathrm{GrF}$ 
pores $(580 \mu \mathrm{m})$. An infiltration factor $\left(\mathrm{I}_{\mathrm{f}}\right)$ equation proposed by our group was used to determine the infiltration behavior of SMP epoxy through GrF [78]:

$$
\mathrm{I}_{\mathrm{f}}=\frac{\mathrm{d}}{\mu}
$$

where $\mathrm{d}$ is GrF cell size and $\mu$ is SMP epoxy viscosity.

The high value of this factor indicates an excellent infiltration behavior. The SMP epoxyGrF composite in this study demonstrated infiltration factor of $\sim 23 \mu \mathrm{m} / \mathrm{cP}$. The free-flow infiltration of SMP epoxy through GrF makes facile processing of GrF-based SMP epoxy composites possible. Figures $28 \mathrm{a}$ and $\mathrm{b}$ show fractured surface features of SMP epoxy-GrF composites at low and high magnifications. Figure 29a display a cured roughened surface of the composite. Curing of SMP epoxy with diamine crosslinker can influence the interface of GrF and SMP epoxy during and after its polymerization. Effective addition reaction between the reactive groups of the epoxies and diamine allows for complete crosslinking. This is reflected in the interfacial appearance between the SMP epoxy and GrF. Some regions in the SMP epoxy-GrF composite revealed average interfacial spacing of $1.32 \mu \mathrm{m}$ (figure 29b). The presence of interfacial spacing can be due to observed volume shrinkage of epoxy during its curing, where long monomer chains adjust into short polymer chains of SMP epoxy.

However, figure 29a reveals that SMP epoxy percolates through the pores of the GrF. This shows the capability of SMP epoxy in filling through the entire hierarchical, macro-porous architecture of GrF. GrF infiltrated with SMP epoxy shows slight bending of its branch, without a change in its branch-node configuration. This depicts that GrF framework has good structural integrity, making it a suitable sturdy carbon filler in polymer composites. 
Some regions in figure 29 also show SMP epoxy adhering to the GrF branches. The SEM micrographs thus indicate the possibility of phonons and electron transfer SMP to improve shape memory behavior for both the thermally and electrically stimulated GrF reinforced SMP epoxy composites. The ability for SMP epoxy to wet and adhere to interconnected graphene sheets network is necessary to facilitate easy phonons and electrons transfer for its enhanced shape memory response.
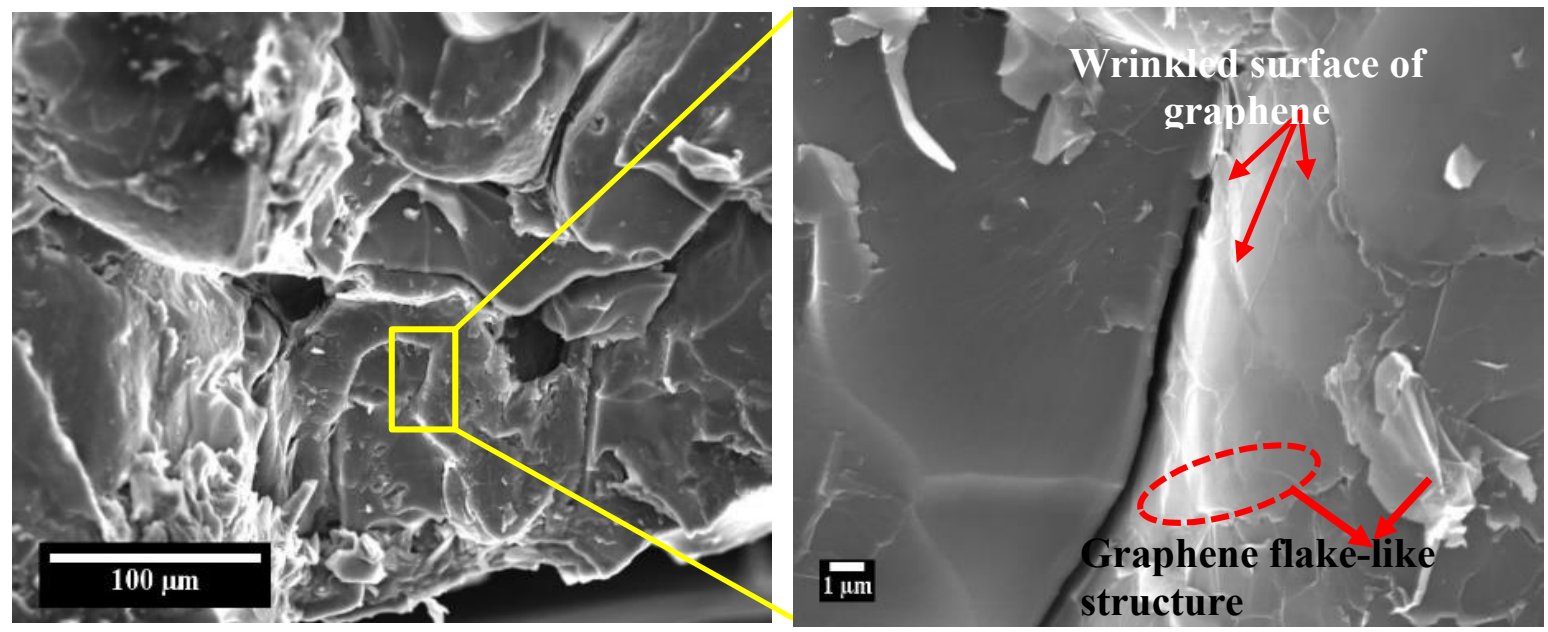

Figure 28: Fracture surface of SMP epoxy-GrF composites revealing wrinkled graphene surface and graphene flake-like structure at (a) low and (b) high magnifications

\subsection{Phase and Bonding Characteristics}

Micro Raman spectroscopy study was conducted on SMP epoxy-GrF composites to mainly examine the interaction of graphene sheets with SMP epoxy chain molecules. Figure 29 shows the Raman measurement of pristine GrF, displaying three major characteristics signatures of graphene sheets. The signatures include $\mathrm{sp}^{2}$ carbon-carbon bonds in-plane vibrations ( $\mathrm{G}$ band), out of plane vibrations due to structural defects (D band), and second disorder band also known as an overtone of D-band (2D band). The significantly low intensity of D compared with intensity of G indicates that the graphene 
sheets are free of defects, as confirmed in previous studies on Raman of graphene [34]. The Raman shift of the 2D peak of pristine GrF used in this study $\left(2704.35 \mathrm{~cm}^{-1}\right)$ is comparable to the 2D peak of typical GrF. It establishes that pristine GrF consists of a network of multilayer graphene sheets. Like pristine $\mathrm{GrF}$, the graphitic signature arising from the stretching of C-C bond remains $1577.04 \mathrm{~cm}^{-1}$ after SMP epoxy infiltrated the graphene foam. It suggests that the bond length of the $\mathrm{C}-\mathrm{C}$ bond that gives graphene identity is intact and physical interaction mainly exists between SMP epoxy and infiltrated GrF. The D and 2D peaks (disorder and second disorder band) of pristine GrF are found at 1315.64 and $2704.35 \mathrm{~cm}^{-1}$, respectively. However, impregnation of GrF with SMP epoxy considerably shifted the D and 2D peaks to the right with an assigned value of 1343.24 and $2709.32 \mathrm{~cm}^{-}$ ${ }^{1}$, respectively. This implies that there is physical interaction of the back-bone chain of SMP epoxy with a network of graphene sheets. Such interaction could have led to an adjustment in bond length of the SMP epoxy molecules. Fourier transforms infrared (FTIR) analysis further confirms the bond length adjustment in SMP epoxy after GrF addition. Figure 30 shows the FTIR spectra of SMP epoxy, GrF, and SMP epoxy-GrF composite. Absorption peaks in SMP epoxy spectrum are revealed at 3348, 2970, 1535, and $1100 \mathrm{~cm}^{-}$ 1 which are attributed to stretching and bending vibrations from $\mathrm{O}-\mathrm{H}, \mathrm{C}-\mathrm{H}, \mathrm{N}-\mathrm{H}$, and C$\mathrm{O}$, respectively [143]. The spectrum of GrF shows characteristics peaks of some functionalities such as C-O at $1100 \mathrm{~cm}^{-1}$; C-H at $2970 \mathrm{~cm}^{-1}$ and O-H at $3348 \mathrm{~cm}^{-1}$ [144]. SMP epoxy-GrF absorption band shows that SMP epoxy peaks are pronounced, overshadowing the weak peaks of GrF. From the FTIR spectra, it could be seen that interfacial intermolecular interactions occur between functionalities on GrF and SMP epoxy. The O-H group from graphene, which can easily be attached to graphene edges, 
initiate electrostatic attractions with $\mathrm{O}-\mathrm{H}$ and $\mathrm{N}-\mathrm{H}$ groups from SMP epoxy. This creates hydrogen bonding, a form of intermolecular force between the graphene and SMP epoxy. Hydrogen bonding interactions between hydroxyls and hydroxyl-amide group result in the formation of hydrogen bond strengths. Typical intermolecular hydrogen bond strength between hydroxyl and amide groups is $29 \mathrm{~kJ} / \mathrm{mol}$, and hydrogen bond between two hydroxyl and hydroxyl groups is $21 \mathrm{~kJ} / \mathrm{mol}$ [145]. Thus, these strong hydrogen bond attractions between these functionalities could account for the good interfacial bonding witnessed in SMP epoxy-GrF composites.

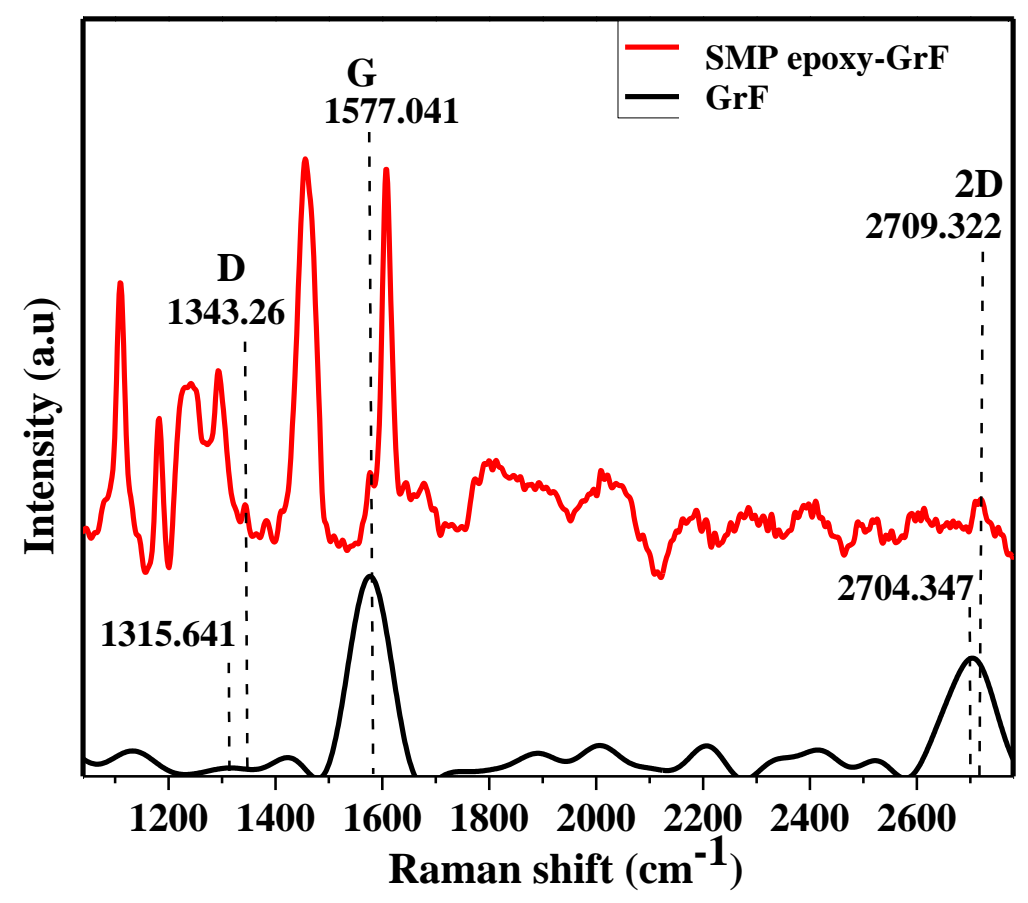

Figure 29: Raman spectrum of GrF and SMP epoxy-GrF Composite revealing peaks characteristics of $\mathrm{GrF}$ and peak shifts in the composite 


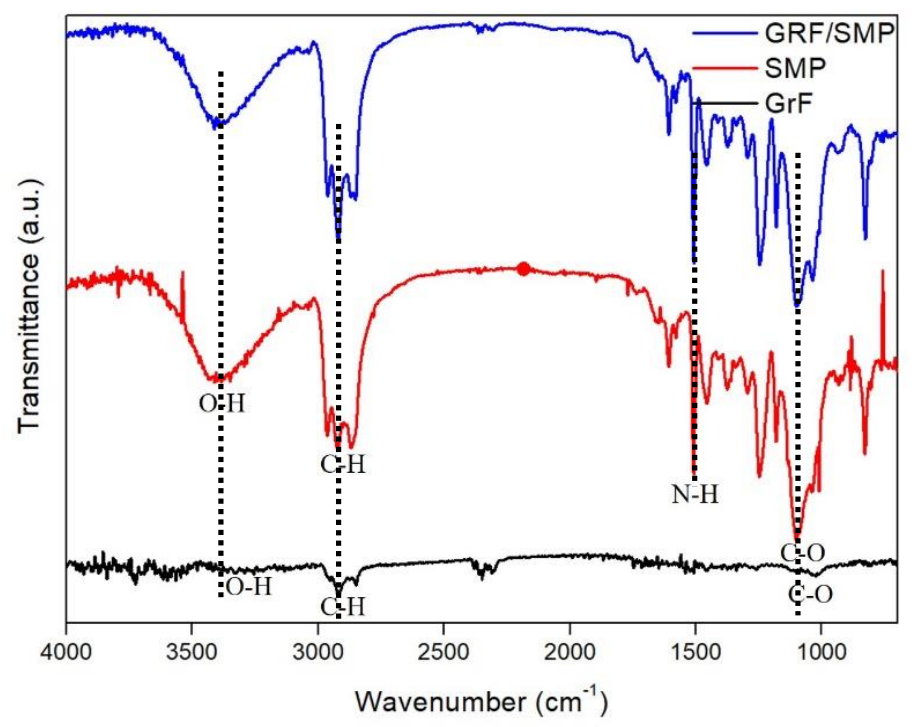

Figure 30: FTIR spectra of GrF, SMP epoxy and SMP epoxy-GrF composite confirming possibility in interfacial interactions between functional groups on SMP epoxy and $\mathrm{GrF}$

\subsection{Thermal characteristics}

Figure 31 presents the thermal transition characteristics of SMP epoxy-GrF composites. Tg of SMP epoxy displayed in figure 31 is $42^{\circ} \mathrm{C}$. GrF filler in SMP epoxy increased the $\mathrm{Tg}$ of 0.13 wt.\% SMP epoxy-GrF sample to $50^{\circ} \mathrm{C}$. This implies that $\mathrm{GrF}$ addition increases the Tg of SMP epoxy-GrF sample composites by $19 \%$ over epoxy SMP. 


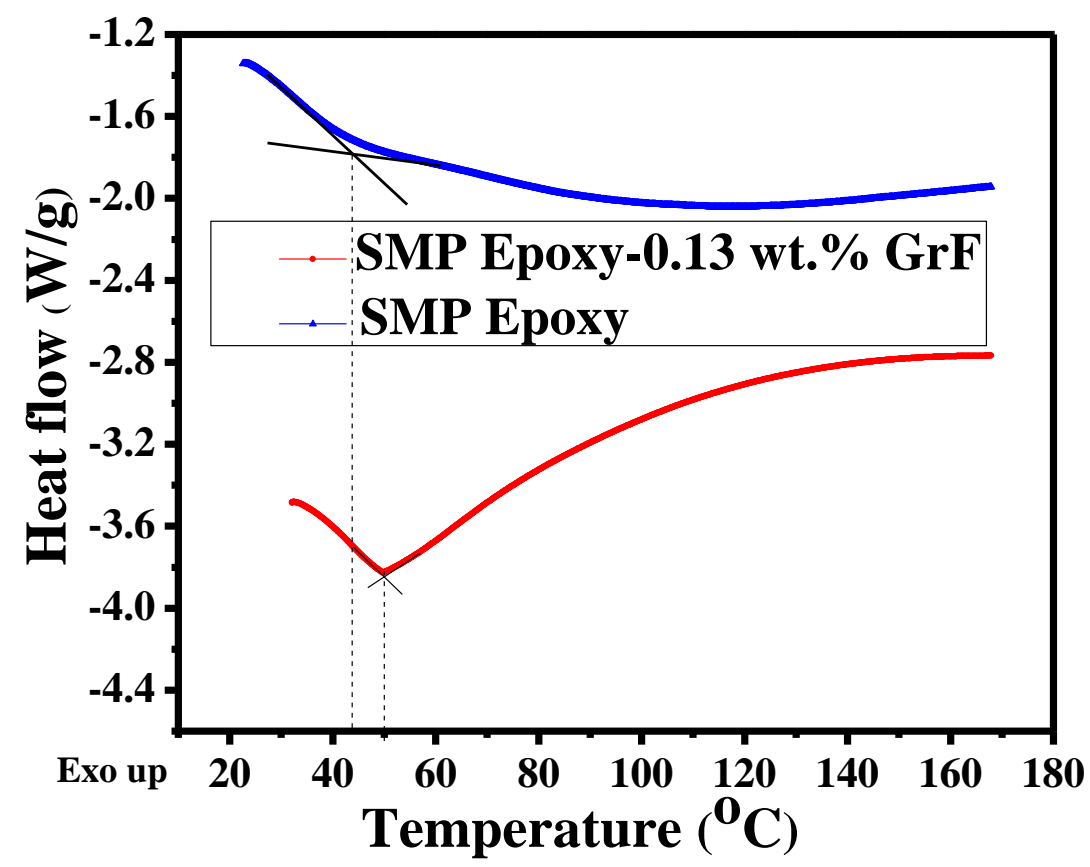

Figure 31: Differential Scanning Calorimetry of SMP epoxy and SMP epoxy-0.13 wt.\% $\mathrm{GrF}$ composite displaying their glass transition behaviors

3D interconnected framework that GrF provides within SMP epoxy matrix initiates entanglement of the terminal hydroxyl group in the side chains of SMP epoxy. This consequently immobilizes the surrounding chains of SMP epoxy molecules around its terminal group, preventing its chain crosslinking [71]. More physical interaction of SMP epoxy terminal group with the 3D graphene sheets network causes the backbone SMP epoxy chain to wrap around the branches and nodes of GrF. The interaction leads to more obstruction in the degree of movement of the individual SMP epoxy chain past one another. Consequently, it requires greater thermal energy to drive the mobility of the confined SMP epoxy chains from its glassy state to a rubbery state. The physical crosslinking results in enhanced $\mathrm{Tg}$ of SMP epoxy-GrF composites. Increase in $\mathrm{Tg}$ is very necessary because $\mathrm{Tg}$ of SMP epoxy needs to be higher than typical environmental temperature. This helps to 
prevent untimely trigger of shape recovery of SMP epoxy. It could also preclude shape recovery deterioration of SMP epoxy after thermal cycling. It is important to note that higher or lower $\mathrm{Tg}$ value is desirable based on the type of application. For aerospace and structural applications such as morphing of wing and microrobots [117], [146], higher Tg is needed for robust integrity of the component and to survive typically higher operating temperature.

Besides this, SMP epoxy as an amorphous polymer exhibited a broad width of $\mathrm{Tg}$ on the DSC curves (figure 31). However, dramatic narrow Tg was revealed with 0.13 wt.\% GrF addition into SMP epoxy. The sudden change in the thermal transition (Tg) width of SMP epoxy-GrF composites depends on the chain conformations of SMP epoxy [147]. Integration of the GrF in SMP epoxy matrix results in a change in the orientations of SMP epoxy chains. This is due to the chain entanglements of SMP epoxy within the interconnected network of graphene sheets. The chain entanglements restrict the formation of branched chains, thereby decreasing the relaxation time distributions associated with the mobility of the SMP epoxy chains. Reduced relaxation time distributions of SMP epoxy lead to sharp width of the thermal transition of SMP epoxy-GrF composites. The thermal transition width can influence shape recovery behavior of SMP epoxy and the composite. In order words, broad transition temperature of SMP epoxy can give rise to its incomplete shape fixing and slower shape recovery. A narrow transition temperature of SMP epoxy$\mathrm{GrF}$, on the other hand, can result in maximum shape recovery rate of the composite. Thus, it suggests that the narrow thermal transition of SMP epoxy-GrF can bring about the faster recovery rate of the composite. This is further corroborated in section 4.4.2. 


\subsection{Thermal actuation of SMP epoxy-GrF composites}

Direct heating using a hot plate, is one of the most recognized stimuli methods to thermally activate SMP epoxy [148]. Hot air from a heat gun was also employed to directly heat and actuate SMP epoxy. Shape memory behavior of SMP epoxy actuated by direct heating is based on dual-component mechanism of SMP epoxy [149]. Figure 32a and b show schematics which describe the shape memory effect of SMP epoxy at the molecular level. SMP epoxy, made up of a structural combination of EPON and NGDE, is heated from room temperature, $T_{1}$, to above the triggering temperature, $T_{2}$. Stress is applied at $T_{2}$ $>\mathrm{Tg}$. This results in the elastic deformation of NGDE chains and storage of energy within the EPON stiff phase.
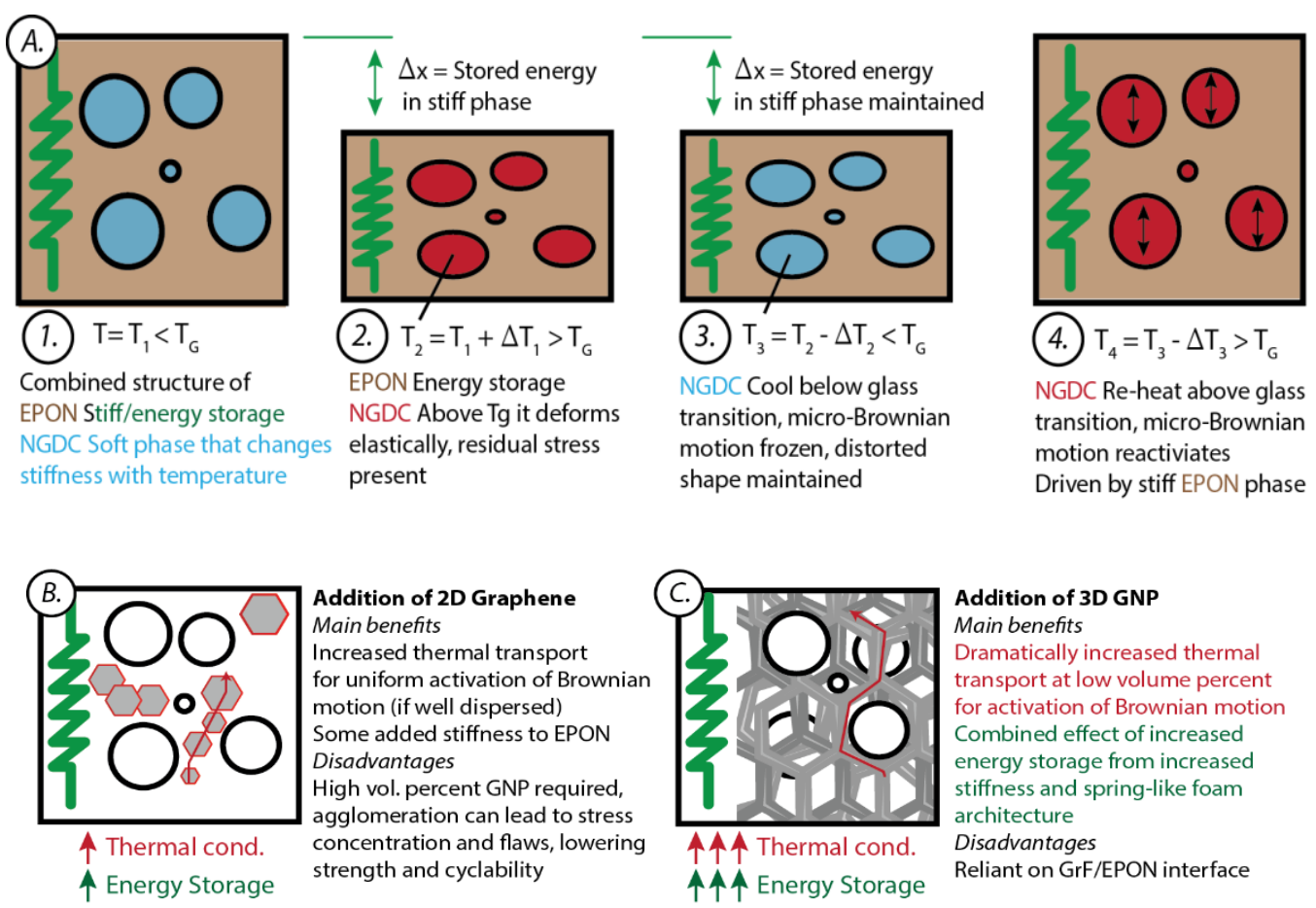

Figure 32: (a) Illustration of the Dual Component Mechanism (DCM) in SMP Epoxy (b) Dual Component mechanisms of enhanced performance for 2D Graphene addition, (c) the mechanisms of improved performance for 3D graphene foam-based additions. 
The temperature of SMP epoxy is then cooled below $\mathrm{Tg}\left(\mathrm{T}_{3}<\mathrm{T}_{\mathrm{g}}\right)$, and the applied stress is removed. The temperature drop, $\mathrm{T}_{3}$, in the SMP epoxy reflects in the cooling of the NGDE polymer segments. The polymer chains, having micro-Brownian motion, are frozen at this stage and allows for the distorted, temporary shape of SMP epoxy. SME of SMP epoxy is afterward triggered when $\mathrm{T}_{3}>\mathrm{T}_{\mathrm{g}}$. This is because the EPON phase propels the full recovery of the distorted polymer molecules when NGDE is reheated (where frozen micro-Brownian motion reactivates). Evaluation of the shape recovery behavior of SMP epoxy-based samples in terms of temporal and thermal gradients is further considered.

\subsubsection{Temporal Gradient Behavior of SMP epoxy-GrF Composite}

Temporal gradient of the SMP epoxy samples are qualitatively assessed when subjected to direct heating source from the hot plate (Figure 33). Figure 33a and b present fold-deploy shape recovery test of SMP epoxy and SMP epoxy-0.75 wt.\% GrF composite, respectively, at $70^{\circ} \mathrm{C}$.

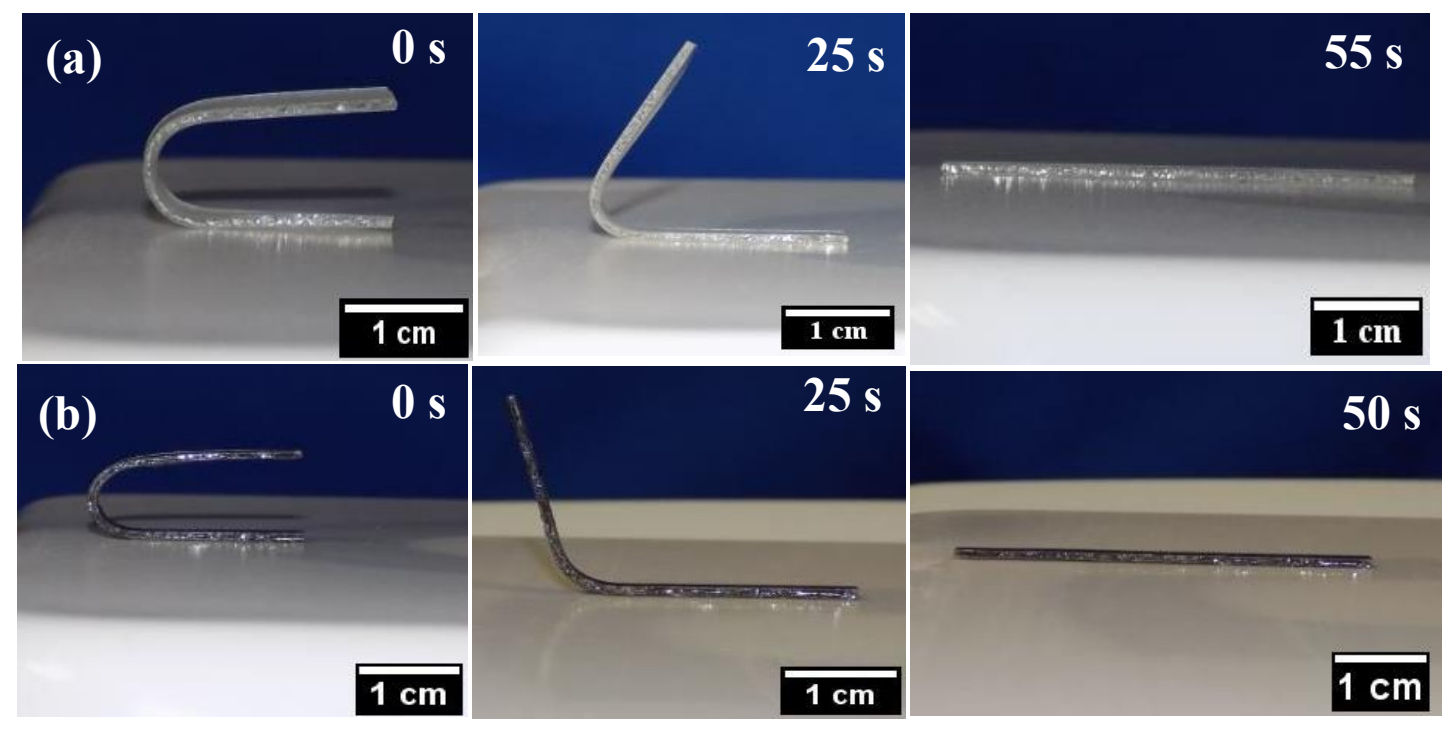

Figure 33: Shape recovery images of (a) SMP epoxy and (b) SMP epoxy-0.75 wt.\% GrF composite triggered by direct heating from the hot plate. 
It is observed that the SMP epoxy composite shows higher temporal gradient than SMP epoxy. Further, based on the shape recovery ratio equation (eqn 4.2), temporal gradient of the SMP epoxy samples are quantified in terms of their recovery angle. It is given as:

$$
\begin{gathered}
\text { Shape recovery ratio: } R_{r}=\frac{\theta_{\text {deformation }-} \theta_{\text {residual }}}{\theta_{\text {deformation }}} \times 100 \\
\theta_{\text {residual }}=\text { residual angle during recovery state } \\
\theta_{\text {deformation }}=\text { angle of bending deformation }
\end{gathered}
$$

Figure 34, deduced from the shape recovery ratio, shows the temporal gradient of folddeploy test of SMP epoxy-GrF composites. The plot illustrates that SMP epoxy and its composite exhibit complete shape recovery behavior. The recovery angle as a function of time provides insight about temporal gradients (Figure 34). It can be observed that from fold-deploy test, SMP epoxy-GrF composite displays higher temporal gradients (recovery rate of 4.93 degree/s) compared with that of SMP epoxy (4.0 degree/s). This is due to seamless phonon and electron transfer offered by branch-node framework of GrF. 


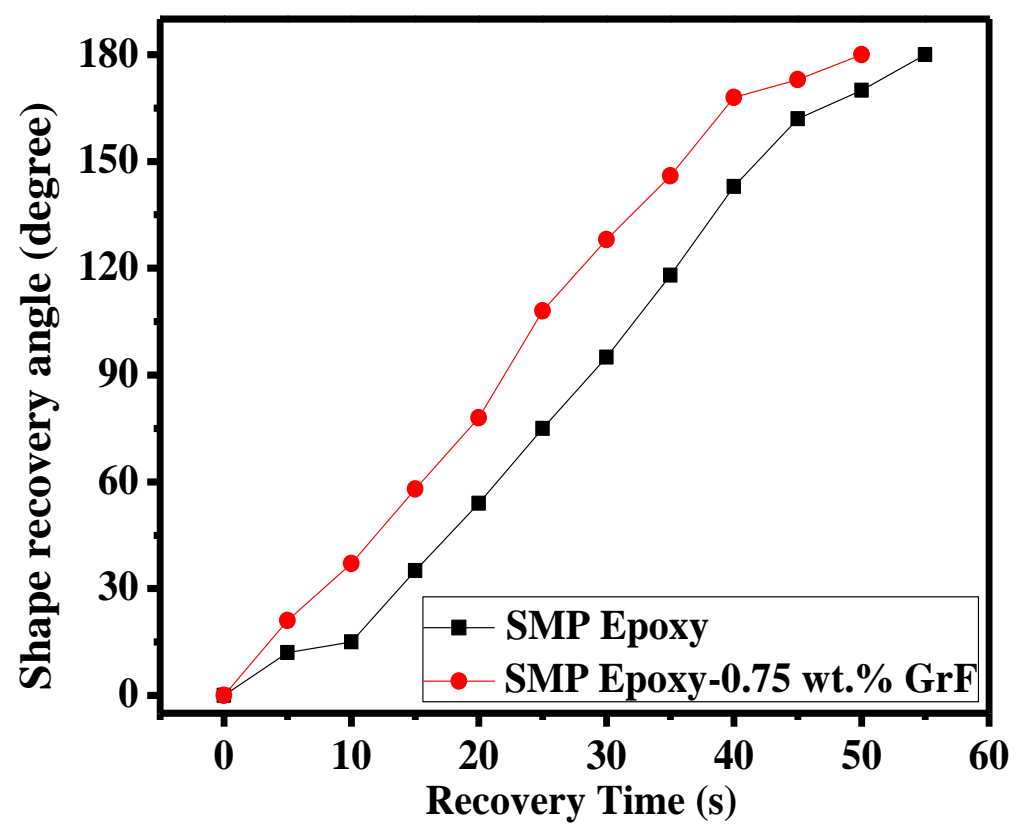

Figure 34: Shape recovery angle as a function of time for SMP epoxy and SMP epoxy$0.75 \mathrm{wt} . \% \mathrm{GrF}$ composite subjected under direct heating from the hot plate

Thus, SMP epoxy-0.75 wt.\% GrF demonstrates a faster recovery rate than SMP epoxy by $0.4^{\circ} / \mathrm{s}$. A t-test was further conducted to establish significance between SMP epoxy and SMP-GrF composite samples. The t value obtained was 38.4. Computation of $p$-value for the shape recovery rate characteristics of the two groups reveals that p-value $<\mathbf{0 . 0 5}$. It indicates that results obtained from recovery rate analysis of the two groups are statistically significant. It further confirms that our research hypothesis that $\mathrm{GrF}$ addition enhances shape recovery behavior of the SMP epoxy composites.

Furthermore, figure 35a and b show recovery images of bent shapes of SMP epoxy and its composite when stimulated by hot air from heat gun having a temperature of $70^{\circ} \mathrm{C}$. SMP 
epoxy and its composite return to their original shape in the recovery time frame of 36 secs when stimulated above their $\mathrm{Tg}$.
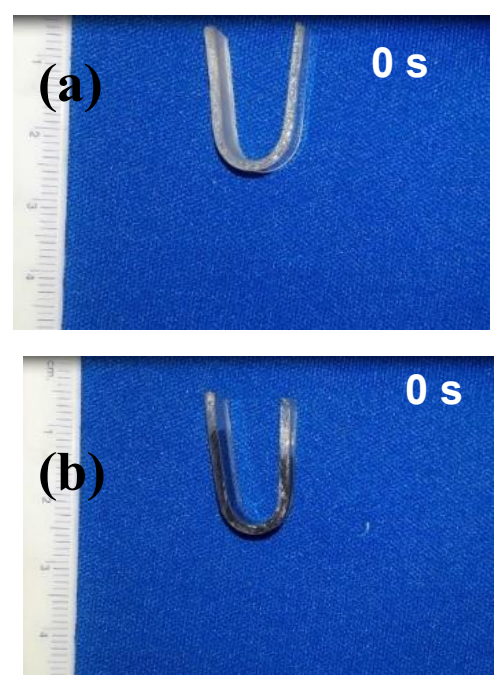

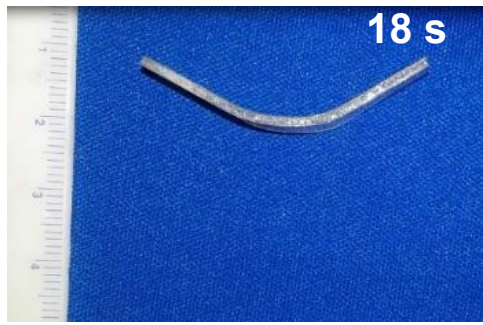

$18 \mathrm{~s}$

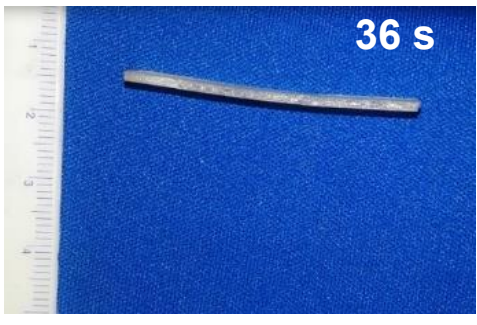

$36 s$

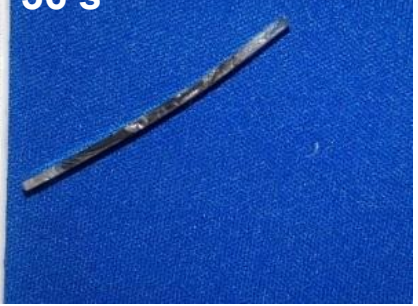

Figure 35: Shape recovery demonstration of thermally actuated (heated air) (a) SMP epoxy and (b) SMP epoxy-0.75 wt.\% GrF composite stimulated by heated air from the heat gun.

From figure 36, SMP epoxy and its composite exhibited average recovery rates of $7.6^{\circ} / \mathrm{s}$ and $8.0^{\circ} / \mathrm{s}$, accordingly. The higher recovery rates of the samples when triggered by hot air suggest uniform heat distribution on the samples than their recovery behavior when subjected to the hot plate. Also, SMP epoxy-GrF displays faster shape recovery rate $\left(0.4^{\circ} / s\right)$ than SMP epoxy. This thus indicates that when different approaches of direct heating of samples are employed, SMP epoxy-GrF has the greater and faster shape recovery characteristics than SMP epoxy. The stiff EPON phase determines the recovery of the SMP epoxy to its permanent, original shape. Its sturdy net-points provide the energy needed to recover SMP epoxy from its temporary shape to its permanent shape. Rigid back bone chain and side chain covalent interactions between the amine and epoxide groups of the EPON segment provide SMP epoxy the capacity that makes it display $97.35 \%$ shape 
recovery. However, the $100 \%$ shape recovery in SMP epoxy- 0.75 wt.\% GrF sample demonstrate the influence of GrF addition in SMP epoxy.

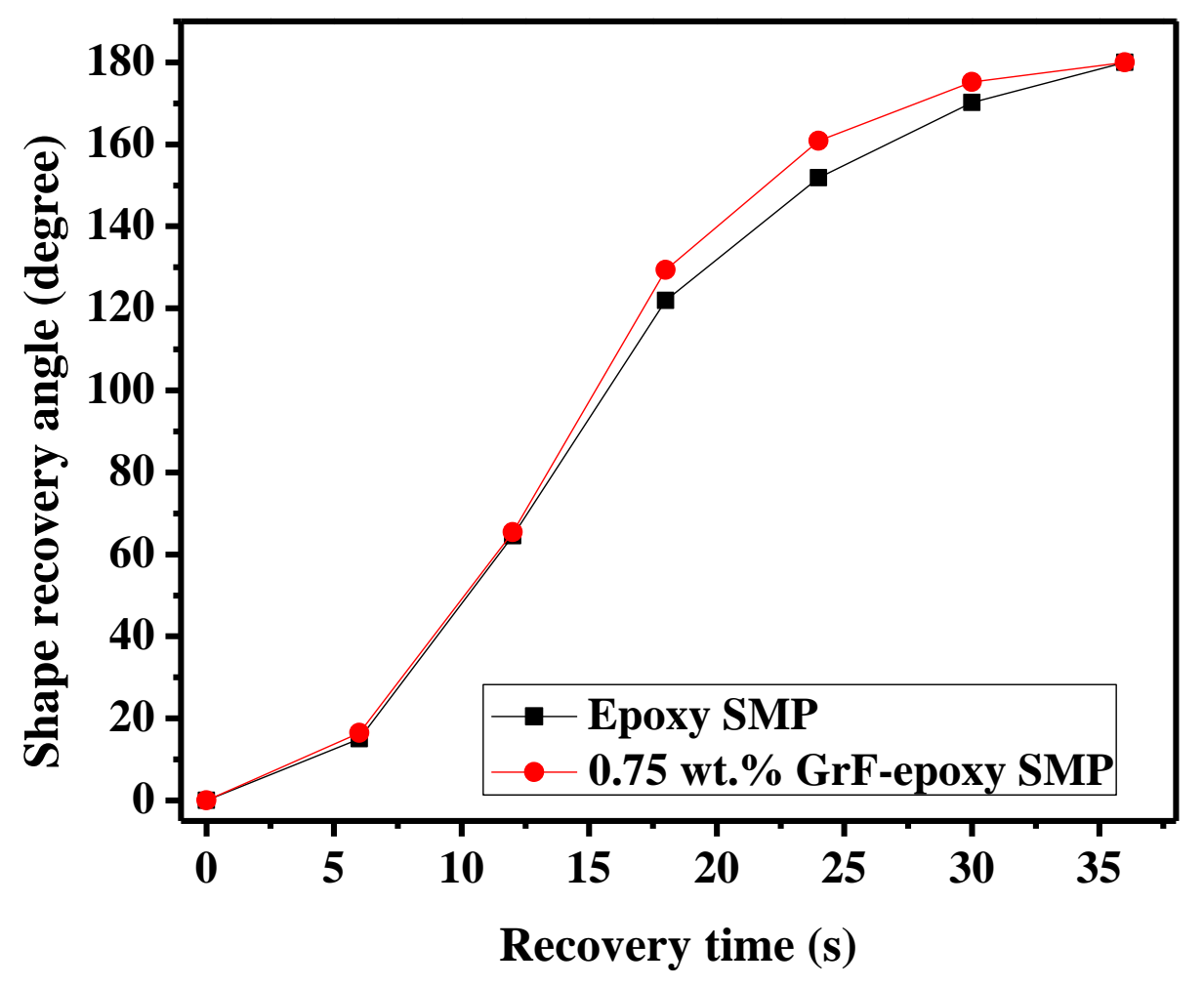

Figure 36: Shape recovery angle as a function of time for SMP epoxy and -SMP epoxy0.75 wt. $\% \mathrm{GrF}$ composite injected with hot air from the heat gun

Since graphene sheets network makes up the framework of GrF, high modulus of graphene sheets can provide more stiffness to the net-points in SMP epoxy. Uniform dispersion of GrF allows the stiffness to be distributed through the entire SMP epoxy matrix. This is due to the good interfacial interaction of the graphene sheets with the SMP epoxy. Existing van der Waals attractive forces between the graphene layers also create spring-like action between the graphene sheets. The attractive intermolecular behavior consequently allows 
GrF to exhibit a capacity for energy storage within the SMP epoxy when deformed into its temporary shape. The energy stored in the GrF is released after actuating the frozen NGDE chain segments. This indicates that $\mathrm{GrF}$ contributes to the maximum recovery noticed in the SMP epoxy-GrF composite. Simultaneous influence of net-point of EPON segment and GrF stiffness and energy storage thus enable the 100\% shape recovery of SMP epoxy-GrF composite.

\subsubsection{Spatial Control of SMP epoxy-GrF Composites via Direct heating}

Like temporal gradient of the SMP epoxy samples, thermal gradients were quantified based on the spatial control via direct heating of the samples (Figure 37a-c). Figure 37 shows Thermal Flir images of direct heat flow through the control (pure SMP) and composite (SMP-GrF) samples at $70^{\circ} \mathrm{C}$ from point $\mathrm{A}(1.0 \mathrm{~cm}$ from the marked origin) to $\mathrm{B}(2.5 \mathrm{~cm}$ from the marked origin)) at $0,5,10$ and $20 \mathrm{~s}$. Quantitative evaluation of the SMP and its composite is further presented in Figure $37 \mathrm{~b} \&$ Table 1.0 and Figure $1 \mathrm{c} \&$ Table 1.1 of this response file, respectively. SMP shows highest thermal gradient from 1 to $2.5 \mathrm{~cm}$ at $20 \mathrm{~s}$ $\left(4^{\circ} \mathbf{C} / \mathbf{c m}\right)$ whereas SMP-GrF composite exhibits highest thermal gradient at $15 \mathrm{~s}$ $\left(5.3^{\circ} \mathbf{C} / \mathbf{c m}\right)$. It is clearly observed that heat propagates faster in $\mathrm{GrF}$ reinforced sample than SMP matrix. This is attributed to uninterrupted pathway created by branch-node framework of GrF. It is important to note that faster heat propagation through $\mathrm{GrF}$ framework enables the composite temperature from point A to B to move towards equilibrium after $10 \mathrm{~s}$ while SMP epoxy still displays thermal gradient after $10 \mathrm{~s}$ (Figure 1d). 


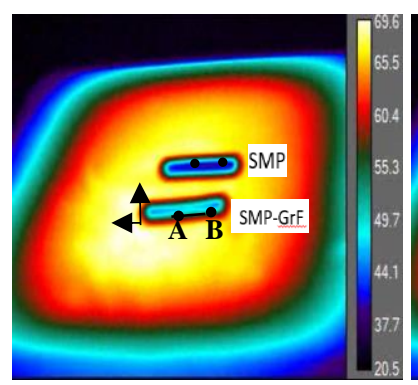

O s

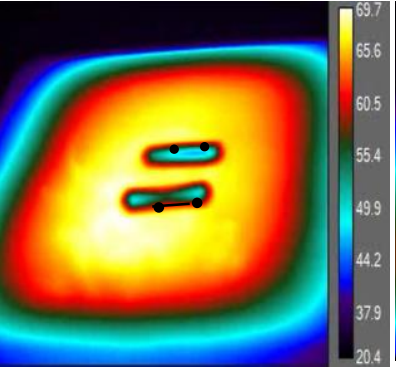

$5 \mathrm{~s}$

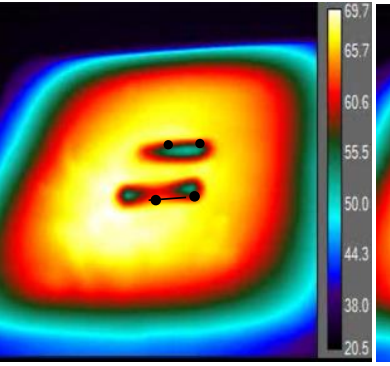

$10 \mathrm{~s}$

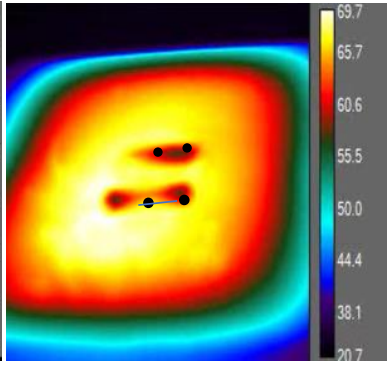

$20 \mathrm{~s}$

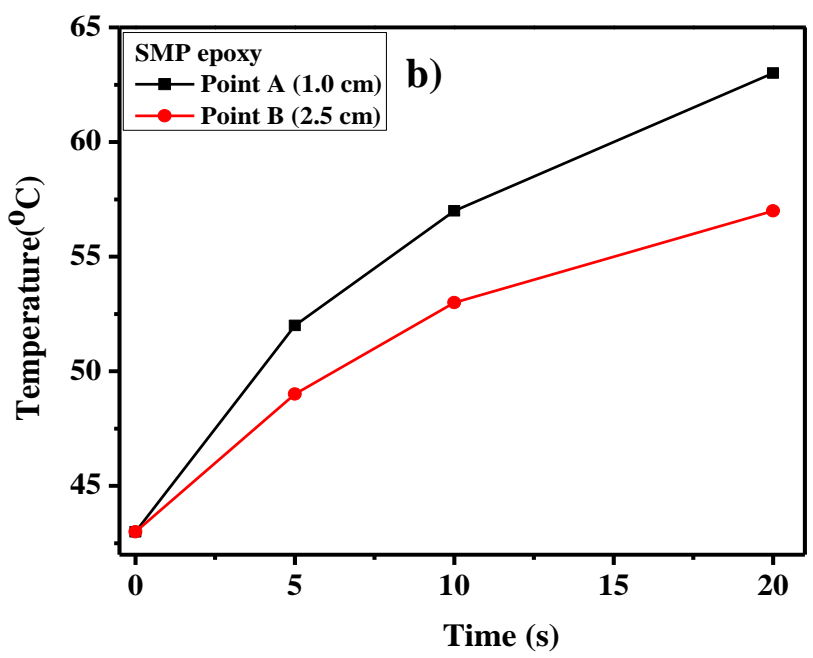

Table 4.0. Thermal gradient of direct heating of SMP epoxy

\begin{tabular}{|c|c|}
\hline At time, $t(s)$ & $\frac{d T}{d x}\left({ }^{o} \mathrm{C} / \mathrm{cm}\right)$ \\
\hline 0 & 0 \\
\hline 5 & 2 \\
\hline 10 & 2.7 \\
\hline 20 & 4 \\
\hline
\end{tabular}




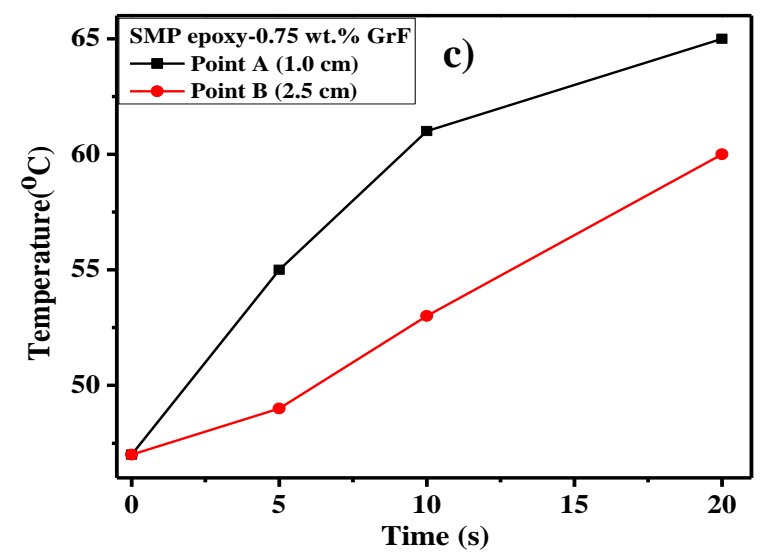

Table 4.1. Thermal gradient of direct heating SMP epoxy-GrF

\begin{tabular}{|c|c|}
\hline At time, $\mathbf{t}(\mathrm{s})$ & $\frac{d T}{d x}\left({ }^{\circ} \mathrm{C} / \mathrm{cm}\right)$ \\
\hline 0 & 0.0 \\
\hline 5 & 4.0 \\
\hline 10 & 5.3 \\
\hline 20 & 3.3 \\
\hline
\end{tabular}

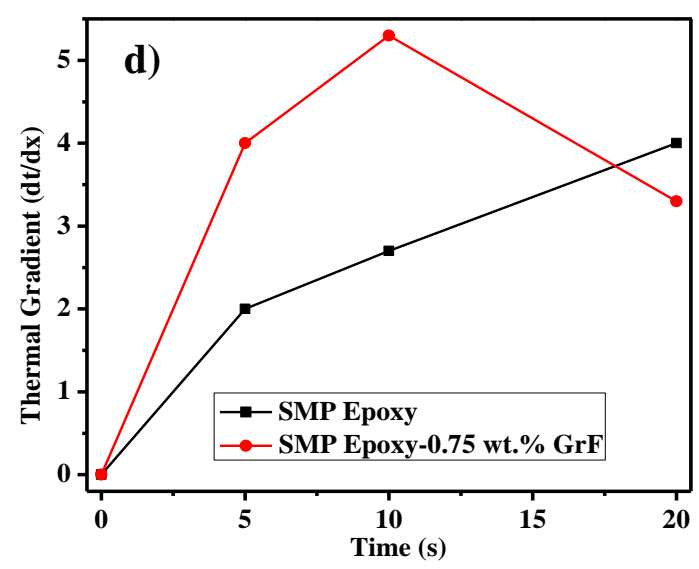

Figure 37. Thermal Flir imaging of SMP-GrF samples. a) stimulated by direct heating (hot plate); thermal gradient of b) direct heating of SMP; c) direct heating of SMP-GrF 
composite showing special control via GrF filler d) Thermal gradient of thermally stimulated SMP Epoxy and SMP Epoxy-GrF Composite as a function time, at $70^{\circ} \mathrm{C}$

Also, thermal conductivities of SMP epoxy and SMP epoxy-GrF composite at $70^{\circ} \mathrm{C}$ were obtained from flash diffusivity method. The conductivity values of SMP epoxy and SMP epoxy-0.5 wt.\% GrF are $0.189 \mathrm{~W} / \mathrm{m}-\mathrm{k}$ and $0.296 \mathrm{~W} / \mathrm{m}-\mathrm{k}$, respectively (figure 38). It implies that the composite has thermal conductivity value which is approximately $57 \%$ greater than that of SMP epoxy. The higher thermal conductivity of the composite further accounts for its $0.3^{\circ} / \mathrm{s}$ increase in recovery rate than that of SMP epoxy. The conductivity results thus establish that the thermal conductivity of GrF provides a higher recovery rate of SMP epoxy-GrF composite.

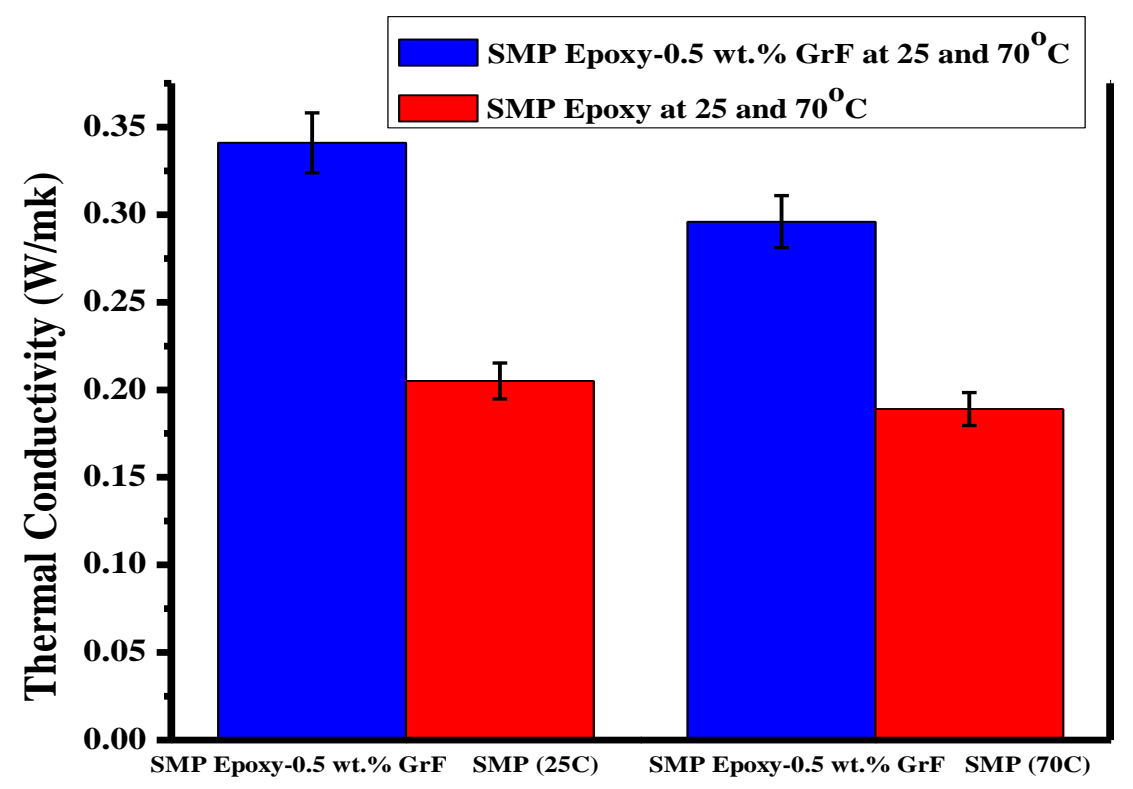

Figure 38: Thermal conductivity behavior of SMP Epoxy and SMP epoxy-0.5 wt.\% GrF at 25 and $70^{\circ} \mathrm{C}$ 


\subsubsection{Effect of switching temperatures on the shape recovery rate of SMP epoxy and SMP epoxy-GrF composite}

Switching temperatures for shape recovery refers to $\mathrm{Tg}$ and temperatures around the Tg region. They are essential parameter in the thermal actuation of SMP epoxy and SMP epoxy-GrF composite. Figure 39 reveals the evolution and trend of the shape recovery rate of SMP epoxy and SMP epoxy-GrF at different switching temperatures. It is notable that for all the switching temperatures, the recovery rate of SMP epoxy-GrF is faster than the SMP epoxy. This attests that GrF addition in SMP epoxy-GrF allows heat conduction in the SMP epoxy-GrF composite due to phonon transfer across the node-branch interconnected network. GrF provides efficient heat transfer that contributes to the unlocking of NGDE chain molecules. The heat conduction within the graphene foam enables the stored energy in both GrF and EPON molecules to drive the shape recovery of graphene foam-epoxy SMP composite. Comparison of recovery rates of SMP epoxy and SMP epoxy-GrF composite for the switching temperatures is presented in figure 39. Figure

39 reveals that the highest recovery rate is obtained at the switching temperature of $\operatorname{Tg}+20^{\circ} \mathrm{C}$. It suggests that the injection of thermal energy at $\mathrm{Tg}+20^{\circ} \mathrm{C}$ provides enough heat distribution needed to trigger shape recovery in SMP epoxy and its composite. This positions the switching temperature, $\mathrm{Tg}+20^{\circ} \mathrm{C}$, to be the required trigger temperature for shape recovery of SMP epoxy-GrF and SMP epoxy-GrF composite. 


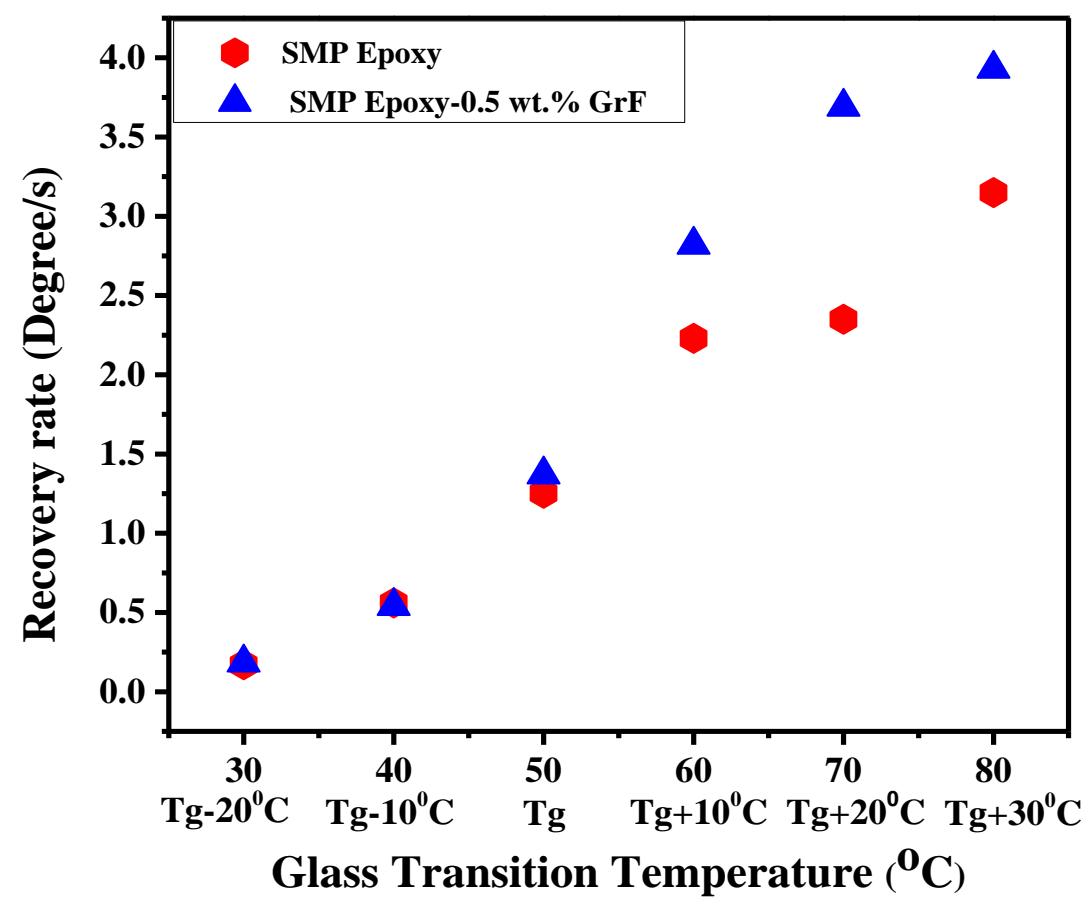

Figure 39: Shape recovery rate of SMP epoxy and SMP epoxy- $0.5 \mathrm{wt} . \% \mathrm{GrF}$ as a function of switching temperatures

Furthermore, the narrow Tg width of SMP epoxy-GrF shown in figure 31 also influences the recovery rate of SMP epoxy-GrF in figure 39. Restriction in the mobility of the soft segment NGDE chains can bring about short relaxation time distribution, leading to narrow glass transition width. GrF does not only physically crosslink with the net-points; it can also impede the movement of NGDE chains and limit its overall chain length for interaction. Consequently, graphene foam addition in SMP epoxy-GrF displays narrow width of Tg transition. This thereby contributes faster shape recovery behavior in SMP epoxy-GrF composite than SMP epoxy-GrF. 


\subsubsection{Influence of thermo-mechanical cycles on the glass transition of SMP epoxy-0.5 wt.\% GrF composite}

Figure 31 has previously shown that integration of GrF in SMP epoxy-GrF can increase the Tg of SMP epoxy-GrF composite. T-M cycling can also influence the Tg of the composite. Figure 40 reveals that $\mathrm{Tg}$ behavior of the composite is controlled by applied T-M cycles.

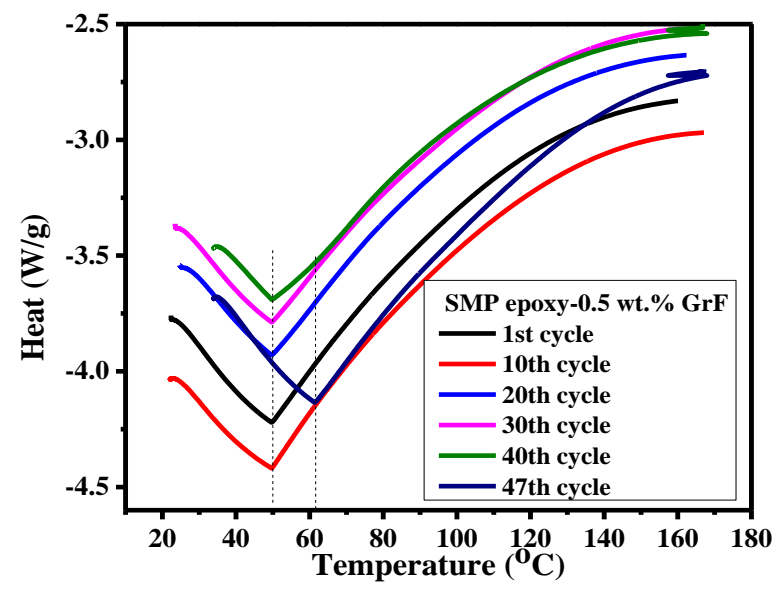

Figure 40: Glass transition behavior of SMP epoxy- $0.5 \mathrm{wt} . \% \mathrm{GrF}$ as a function of T-M cycles

1st to 30th applied T-M cycles do not show a change in Tg. However, the 47th T-M cycle demonstrates an increase in $\mathrm{Tg}$ from $50^{\circ} \mathrm{C}$ to $58^{\circ} \mathrm{C}$. This indicates that the $\mathrm{Tg}$ of the composite increase by $16 \%$. The constant Tg of SMP epoxy- 0.5 wt. $\%$ GrF observed from 1st to 40th T-M cycles could be due to constrained entanglements of GrF with sidechains of SMP epoxy. As the T-M cycle increases, it facilitates more physical interaction of GrF with the backbone chain of SMP epoxy. The increased physical interaction confines the internal rotation of the backbone chain at $47^{\text {th }} \mathrm{T}-\mathrm{M}$ cycle. This leads to the $16 \%$ increase in Tg of SMP epoxy- 0.5 wt. $\%$ GrF at 47th T-M cycle. T-M cycling of SMP epoxy- 0.5 wt. $\%$ 
GrF composite can thus enable the tailoring the $\mathrm{Tg}$ of the composite. This allows the temperature range to be widened for SMP epoxy composite applications.

\subsection{Electrical actuation of epoxy SMP and graphene foam-epoxy SMP composite}

The use of the direct thermal source for actuation of shape change in SMP epoxy limits its practical applications. Induction of heat through electricity (indirect heating) as an alternative trigger method in epoxy SMP is essential. Graphene, as a carbon-based filler, has been considered to electrically trigger epoxy SMP [150]. However, graphene's high electrical conductivity can be compromised because of its high inter sheet contact resistance and restacking issues. Hence, 3D GrF filler is considered to electrically stimulate SMP epoxy for improved shape memory behavior of the composite.

Indirect heating of SMP epoxy and SMP epoxy-GrF composite are conducted to examine their shape recovery behavior and to determine is temporal (figure 41) and thermal gradients (figure 42). SMP epoxy and its composite subjected to Joule heating at applied currents of (0.1-0.4 A). Thermal Flir images, as shown in figure 42, demonstrates the qualitative evaluation of the shape recovery of the electrically actuated samples. No shape recovery is observed in SMP epoxy. Complete shape recovery is noticed in SMP epoxyGrF composite at 0.4 A. Figure 41 presents quantification of the temporal gradient of the electrically actuated SMP epoxy samples at 0.4 A. No temporal gradient was shown during electrical actuation of SMP epoxy. The composite, however, showed recovery rate of 5.67 degree/s between recovery time 2 and $8 \mathrm{~s}$. Absence of heat flow through current-induced SMP epoxy is further confirmed from the infra-red (IR) thermal image (figure 42a). The images shows constant surface temperature of SMP epoxy from 0 to $25 \mathrm{~s}$. This attests that no heat is generated from the $0.4 \mathrm{~A}$ current. In order words, current-induced heat is absent 
within the SMP epoxy. It suggests that it is impossible to electrically induce SMP epoxy to exhibit shape recovery because it is non-electrically conductive. GrF addition, however, enables SMP epoxy to be actuated by an electrical means. GrF reinforcement serves as a pathway that allows conduction of current-induced heat in SMP epoxy matrix. Applied currents at 0.1-0.4 A are injected through GrF embedded in SMP epoxy. This is illustrated in figure 26 (materials and method section). GrF acts as a resistive element when current passes through it. The resistive heating generated in GrF is induced to the surrounding NGDE and EPON molecular segments of the SMP epoxy matrix. The induced heat from GrF reactivates the micro-Brownian motion of the NGDE molecules. It also agitates the EPON aromatic molecules to discharge the energy stored in the pre-deformed shape. Changes in SMP epoxy chain conformations, due to the heat, drive the recovery of SMP epoxy from its temporary shape to its original shape.

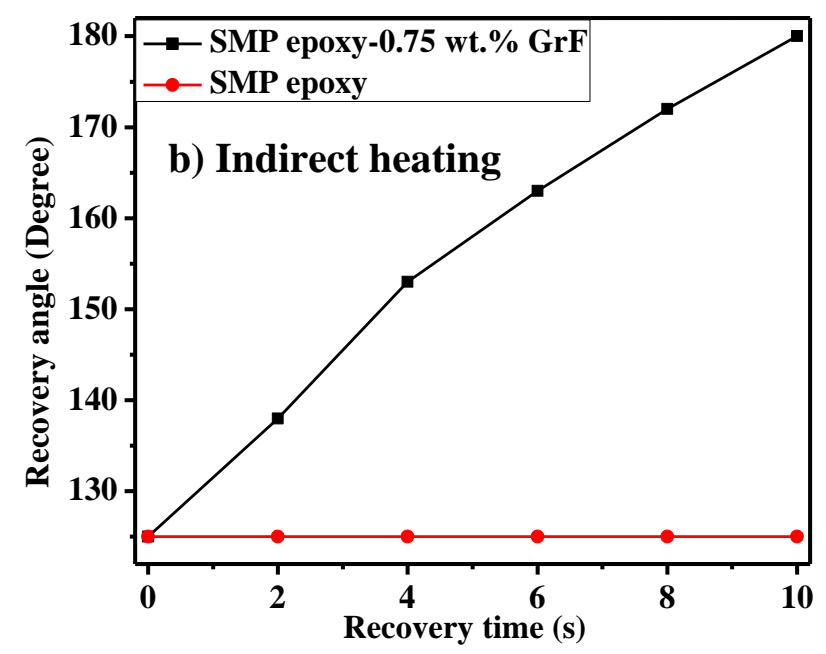

Figure 41. Temporal gradients of fold-deploy tests of SMP epoxy-GrF composites. a) stimulated by direct heating (hot plate); b) triggered by indirect or electrical heating at 0.4 A 


\subsubsection{Spatial Control of SMP epoxy-GrF Composites via electrical (Indirect) heating}

To elaborate on the thermal gradient of electrical heating of SMP epoxy-GrF composite, spatial control via electrical heating is carried out at 0.1-0.4 A (figure $42 \mathrm{~b}$-e and Table 2.0). Thermal Flir images in figure 42b demonstrates current-induced heating through the $\mathrm{GrF}$ filler from point $\mathrm{A}$ ( $1 \mathrm{~cm}$ from the marked origin) to point $\mathrm{B}(5 \mathrm{~cm}$ from the marked origin) at 0,10 and $25 \mathrm{~s}$. GrF spatially controls heat propagation through distance $\mathrm{AB}$ when dose of currents $(0.1-0.4 \mathrm{~A})$ is applied. This is noticed from change in heat intensity (GrF glow) along distance $\mathrm{AB}$, at the applied currents. Heat is gradually and increasingly transported through the GrF to the epoxy SMP at the applied currents. The amount of applied electric current injected into GrF in epoxy SMP determines the intensity of heat propagation through $\mathrm{GrF}$ and from GrF to the SMP epoxy matrix. SMP epoxy-GrF composite exhibits lowest thermal gradient $\left(1.38{ }^{\circ} \mathbf{C} / \mathbf{c m}\right)$ from point A to B, at $25 \mathrm{~s}$ and applied current of $0.1 \mathrm{~A}$. The composite also shows highest thermal gradient $\left(31.3{ }^{\circ} \mathrm{C} / \mathrm{cm}\right)$ at $25 \mathrm{~s}$ and $0.4 \mathrm{~A}$, as shown in figure $42 \mathrm{f}$ and Table 2.0. The propagation of heat in GrF can be attributed to the branch-node anatomy of the GrF. GrF interconnected framework provides seamless pathways for electron flow and phonon transfer. It is observed that electrical heating of the composite shows greater temperature values at 0.3 and $0.4 \mathrm{~A}$ (figure $42 \mathrm{~d}$ and e) than direct heating of the composite (figure $37 \mathrm{a}$ ). This can be due to heat localization created by electrical heating through $\mathrm{GrF}$, whereas direct heating transport heat to a larger body mass of SMP. 


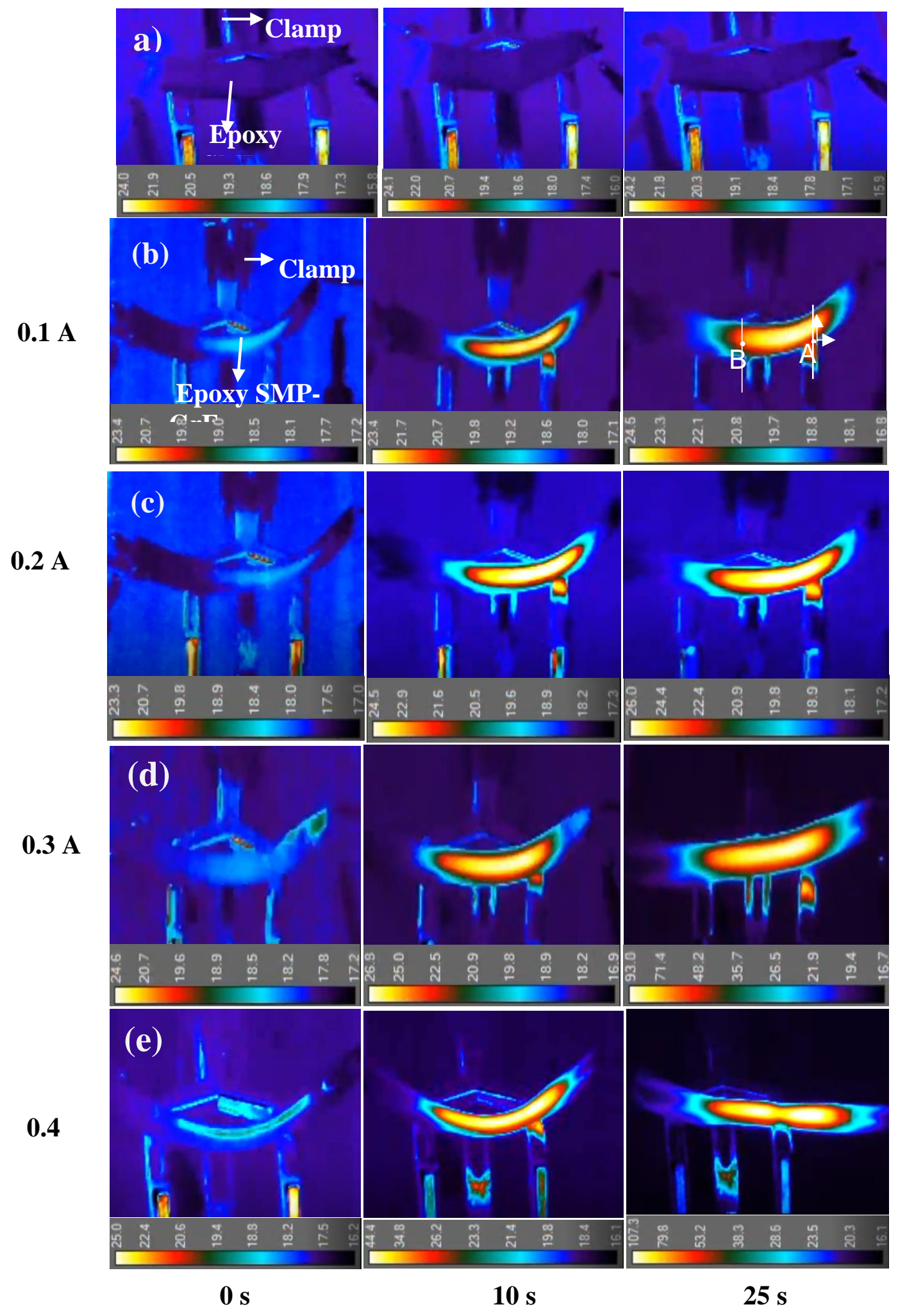




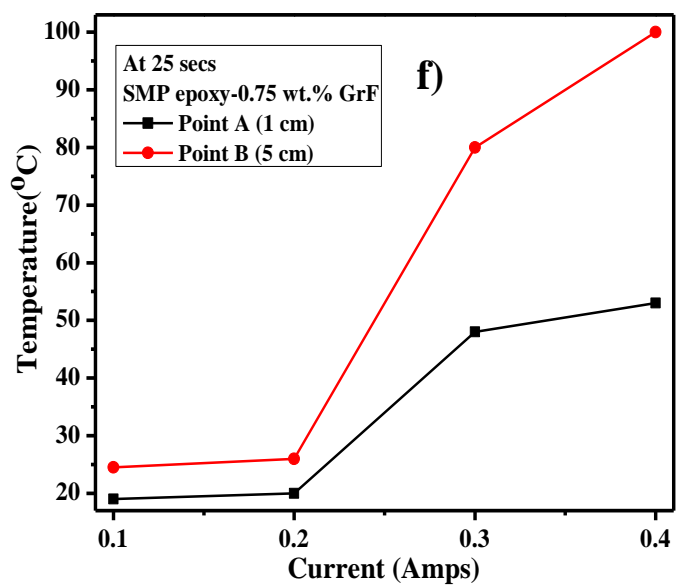

Table 4.2. Thermal gradient of direct heating SMP epoxy-GrF

\begin{tabular}{|c|c|}
\hline $\begin{array}{c}\text { Current } \\
\text { (Amps) }\end{array}$ & $\begin{array}{c}\frac{d T}{d x} \\
\left({ }^{o} \mathrm{C} / \mathrm{cm}\right)\end{array}$ \\
\hline 0.1 & 1.38 \\
\hline 0.2 & 1.5 \\
\hline 0.3 & 21.3 \\
\hline 0.4 & 31.3 \\
\hline
\end{tabular}

Figure 42. Thermal Flir images of SMP epoxy actuated by indirect (electrical) heating at a) $0.4 \mathrm{~A}$ and SMP epoxy-GrF samples at b) $0.1 \mathrm{~A} \mathrm{c}$ ) $0.2 \mathrm{~A} \mathrm{~d}) 0.3 \mathrm{~A} \mathrm{e}) 0.4 \mathrm{~A} \mathrm{f}$ ) thermal gradient of fold-deploy tests of electrical heating of SMP composite showing spatial control via GrF filler

It is important to note that for most applications, both spatial and temporal gradients are important as shape recovery should happen throughout the component and in a rapid manner. This includes example of unmanned aircraft wings and electrically stimulated 
crawling robot. Spatial control is determined by the uniform dispersion of GrF whereas temporal control is largely dictated by the excellent conductivity of GrF.

As seen earlier, electrical heating results in a significantly high spatial control in SMP epoxy-GrF composite as compared to pure SMP. Whereas, it is already determined that for pure SMP, electrical heating does not result in recovery, hence; temporal control is irrelevant. However, it is noted that change in the shape is dictated by the change in the transformation temperature. Thus, a faster rate to achieve the desired temperature in a specific location would always have an edge. Therefore, while spatial and temporal gradients are both very important, temporal gradient has a slight edge for rapid actuation. It is also important to highlight that a good interface between GrF and SMP epoxy also plays good role in the spatial and temporal gradient behavior. Good interface could facilitate thermal boundary conductance of the composite. This could contribute to heat transfer from graphene foam to the epoxy SMP. Further, interfacial thermal resistance (ITR) between GrF and SMP epoxy could influence the shape recovery of SMP epoxy-GrF composite. A simple thermal equation that can be used to determine the ITR between GrF and SMP epoxy is shown below [151]:

$$
\mathrm{R}=\frac{\Delta T A}{Q}
$$

Where $\mathrm{R}$ is the resistance at the interface; $\Delta T$ is temperature drop across the interface; $\mathrm{A}$ is the cross-sectional area, and Q is the total heat transfer across the interface. Determination of parameter $\mathrm{R}$ would influence the control of the heat transport across the SMP epoxyGrF interface. The ITR can cause phonon vibrational mismatch and scattering at SMP epoxy-GrF interface during phonon transfer at the interface. It thus suggests that nano- 
scale contact area between GrF and SMP epoxy accounts for shape recovery of electrically actuated SMP epoxy-0.75 wt.\% GrF composites.

\subsection{Proof of concepts of SME in graphene foam-epoxy SMP shapes and bird composites}

Structural versatility of SMP epoxy and its composites can make it a highly demanded smart polymer in engineering applications. Its large strain deformation enables it to be fabricated into different facile shapes; yet, it does not lose its shape memory behavior. SMP epoxy and SMP epoxy-GrF composites are engineered into simple S, L, and inverted U shapes, and accordion shapes. The basic S, L shapes of epoxy SMP and an inverted U-shape of SMP epoxy-GrF sample were stretched out to its temporary shape and triggered back to its original shape by hot water (figure 43a-c). Also, the accordion shapes made of SMP epoxy and SMP epoxy-GrF were fabricated. These shapes were triggered by both hot water and hot air (figure $44 \mathrm{a}-\mathrm{c}$ and figure $45 \mathrm{a}$ and $\mathrm{b}$ ). The shape designs demonstrate that SMP epoxy and SMP epoxy-GrF composite have very high flexibility and adaptability when above its glass transition temperatures. SMP epoxy-GrF accordion shapes further exhibited faster recoverability (approximately two seconds faster) than that of SMP epoxy due to strong thermal and mechanical capabilities of GrF. This suggests that SMP epoxy-GrF composite can be greatly useful in actuators and piezoelectric devices, deployable reflectors and masts, and offer considerable potentials in morphing wing of aircraft owing to its high shape deformability. 

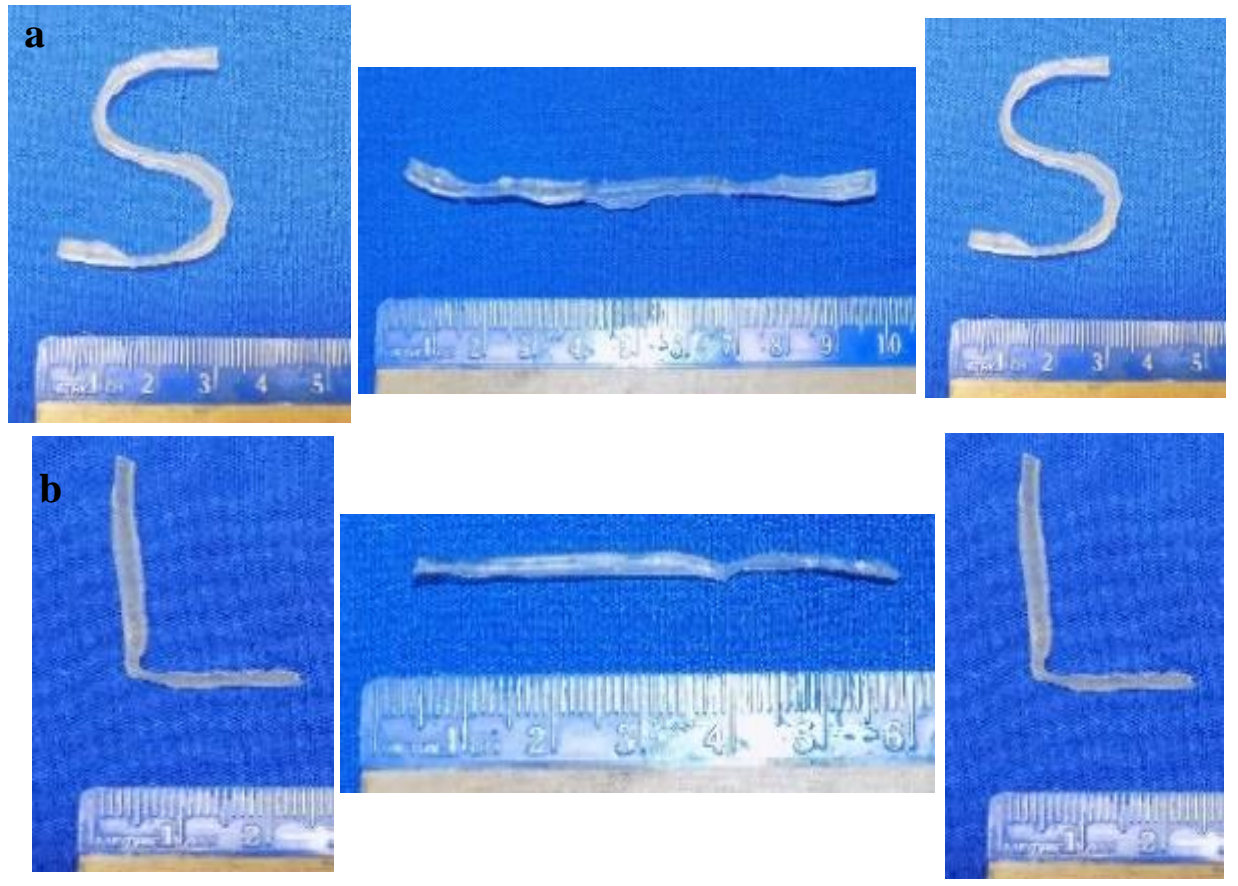

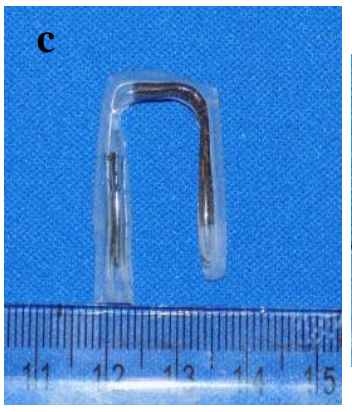

Original shape

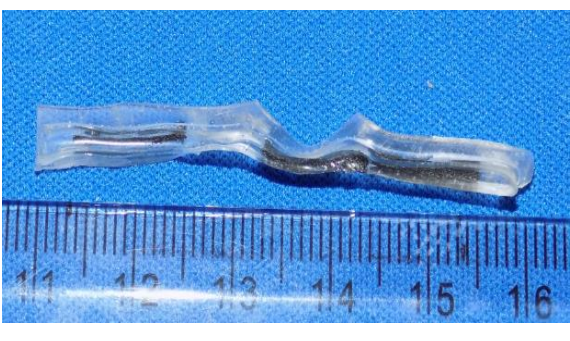

Temporary shape

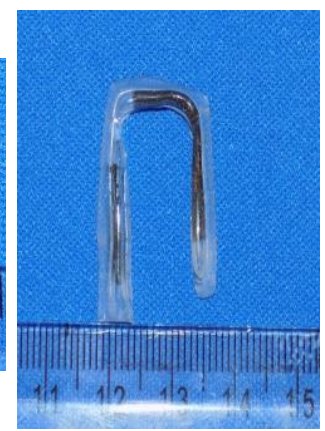

Original

Figure 43: a) Images of epoxy SMP fabricated to S-shape, deformed to thin rectangular shape and recovered back to $S$-shape under the influence external heat stimulus; b) Images of epoxy SMP designed to L-shape. deformed into rectangular shape and recovered back to L-shape when subjected to heat trigger; c) Images of graphene foam-epoxy SMP made into inverted U-shape deformed into irregular rectangular shape and recovered back to inverted U-shape when stimulated by heat 

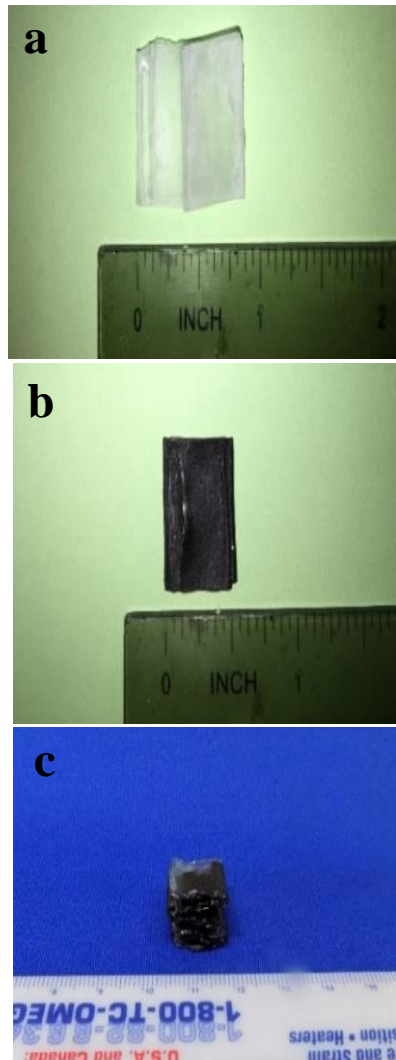

sıацеан • vоім! ụeils pue a

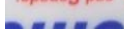
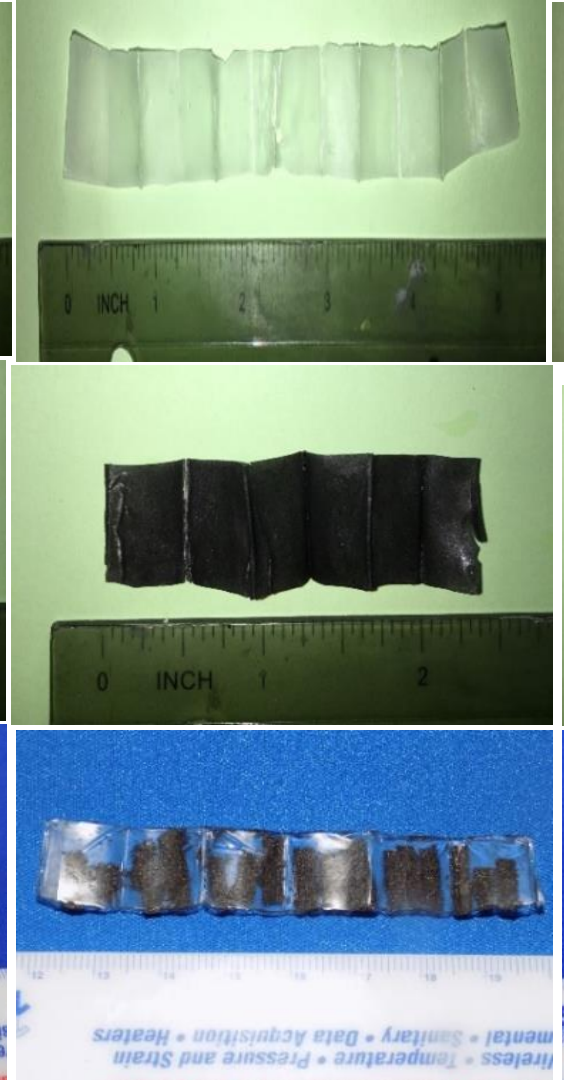

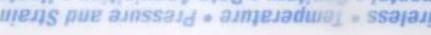
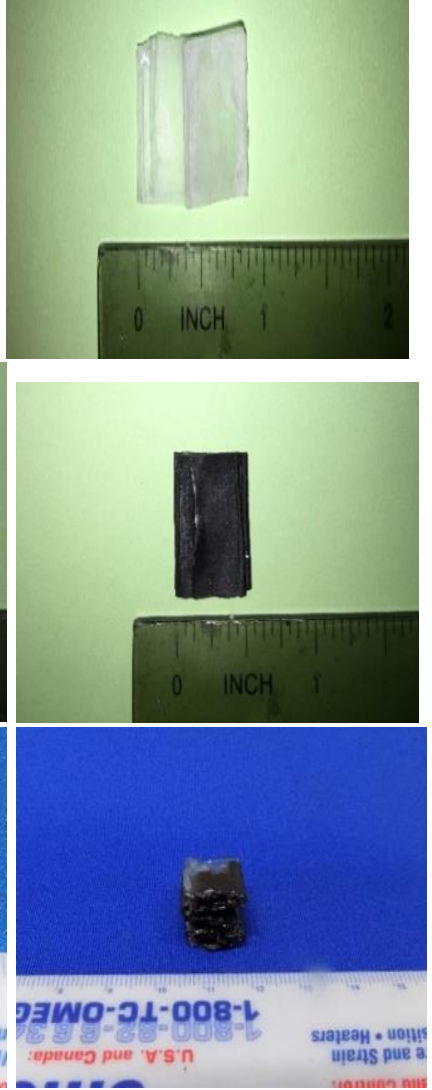

Original Shape

\section{Original shape}

Temporary shape

Figure 44: a) Images of epoxy SMP fabricated into accordion-like shape, stretched out into temporary shape and restored to its original shape under the influence external heat from hot water; b) Images of graphene-epoxy SMP made into accordion-like shape, deformed into temporary shape and recovered to its original shape when triggered by thermal actuation; c) Images of graphene-foam-epoxy SMP composite made into accordion-like shape, deformed into temporary shape and recovered to its original shape when stimulated under heat 


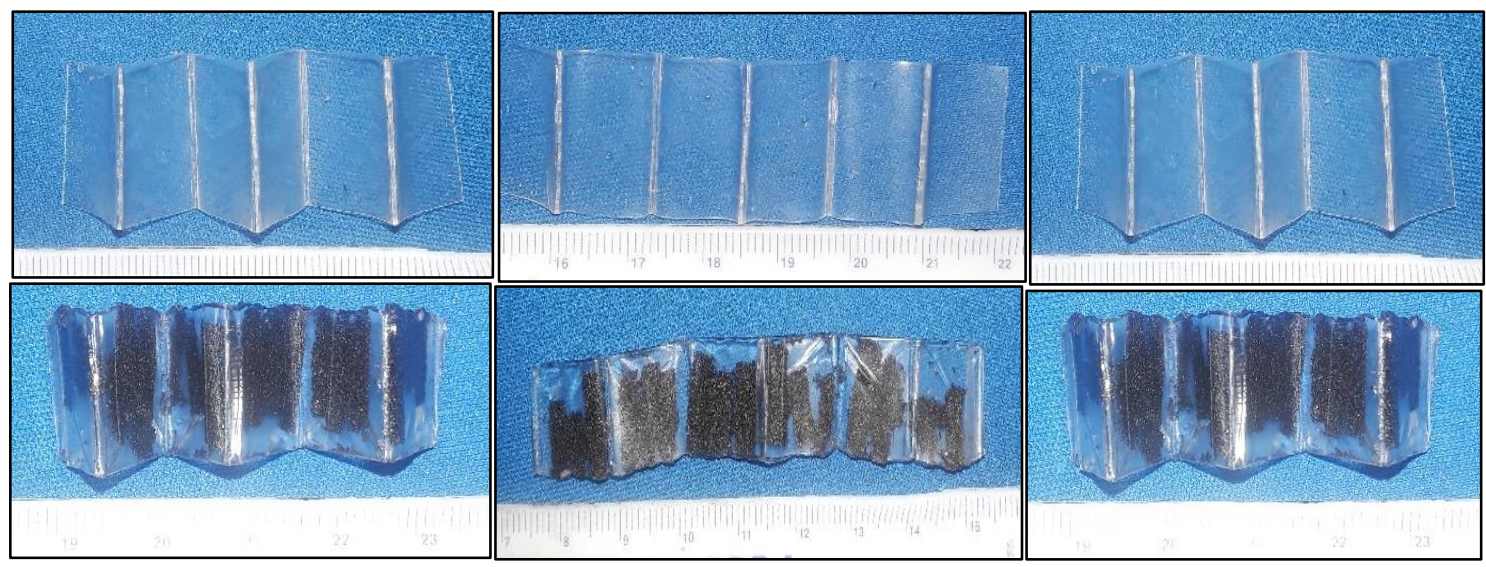

Original shape

Temporary shape

Original Shape

Figure 45: a) Images of epoxy SMP fabricated into accordion-like shape, stretched out into temporary shape and restored to its original shape under the influence hot air; b) Images of graphene foam-epoxy SMP made into accordion-like shape, deformed into temporary shape and recovered to its original shape when triggered by hot air

Furthermore, the good shape deformability of SMP epoxy and high recoverability of SMP epoxy-GrF composites can offer huge potentials in aerospace applications, such as in the morphing wing of aircraft. Inspired by wing of a bird, we fabricated stimulusresponsive SMP epoxy and SMP epoxy-GrF composite birds. Figure 46a and b show predeformed bird wing of SMP epoxy and its composite, respectively. The birds demonstrated highly flexible wings during its deformation above $\operatorname{Tg}\left(70^{\circ} \mathrm{C}\right)$. The wings retained their deformed shape when cooled below its Tg to room temperature. To stimulate the deformed bird wings to recover its original shape, hot water at $70^{\circ} \mathrm{C}$ is used as a trigger source. Hot water provides a steady source of heat during immersion of SMP epoxy and its composite. The recovery of the SMP epoxy and SMP epoxy-GrF bird wings to their original shape is shown in figure 46c and d, respectively. The recovery time of SMP epoxy-GrF bird wing was approximately $1 \mathrm{sec}$ ahead of its pure SMP epoxy bird wing. This is due to the added 
energy storage from GrF in SMP epoxy, which propelled quicker wing recovery of GrF reinforced SMP epoxy bird.

Also, figure 46e illustrates that several T-M cyclic wing deformation of graphene foam-epoxy composite bird revealed surface crack opening on the wing. It is, however, note-worthy that crack closure occurs during recovery of the birds wing to its original shape under heat stimulant (figure 46f). The phenomenon suggests that that structural and dynamic factors contribute to the crack closure behavior of the SMP epoxy-GrF composite. During the formation of surface cracks on the wing of SMP epoxy composite bird, the integrity of the net-points from the EPON phase of SMP epoxy remains intact. This is because the GrF filler serves as a crack-bridging agent, thereby restricting the disintegration of the SMP epoxy net-points. Energy used to initiate crack surface is also transferred through the GrF branch and node hierarchical anatomy. It prevents failure of the wing of SMP epoxy-GrF bird.

Moreover, the dynamic factor of heat causes an adjustment in chain conformations of EPON phase located around the crack region of the wing of SMP epoxy-GrF bird. The chain conformation adjustment of the EPON phase unlocks the stored energy with the EPON phase. Release of stored energy within the EPON segment contributes to the recovery of its original bird wing shape. Conserved energy within the GrF also is released to the crack surface of the SMP epoxy, during the adjustment of EPON chain conformation. The release of store energy helps to drive the crack closure of SMP epoxy-GrF bird composite. SMP epoxy composite bird, as proof of concept, thus points out that SMP epoxy-GrF composite is promising towards the design of morphable wings of aircraft. Furthermore, the concept of reinforcing a shape memory polymer with GrF could be 
extended to a biopolymer such as SMP polyurethane (PU), which is biodegradable and biocompatible. Thermoplastic SMP-PU can find application as neurovascular stent to deliver drug locally which can treat ischemic stroke. The application leverage on shape memory feature during neurovascular stent deployment (stent expansion) to deliver the drug. This is done by using laser to selectively heat the local area and trigger the shape memory function [115]

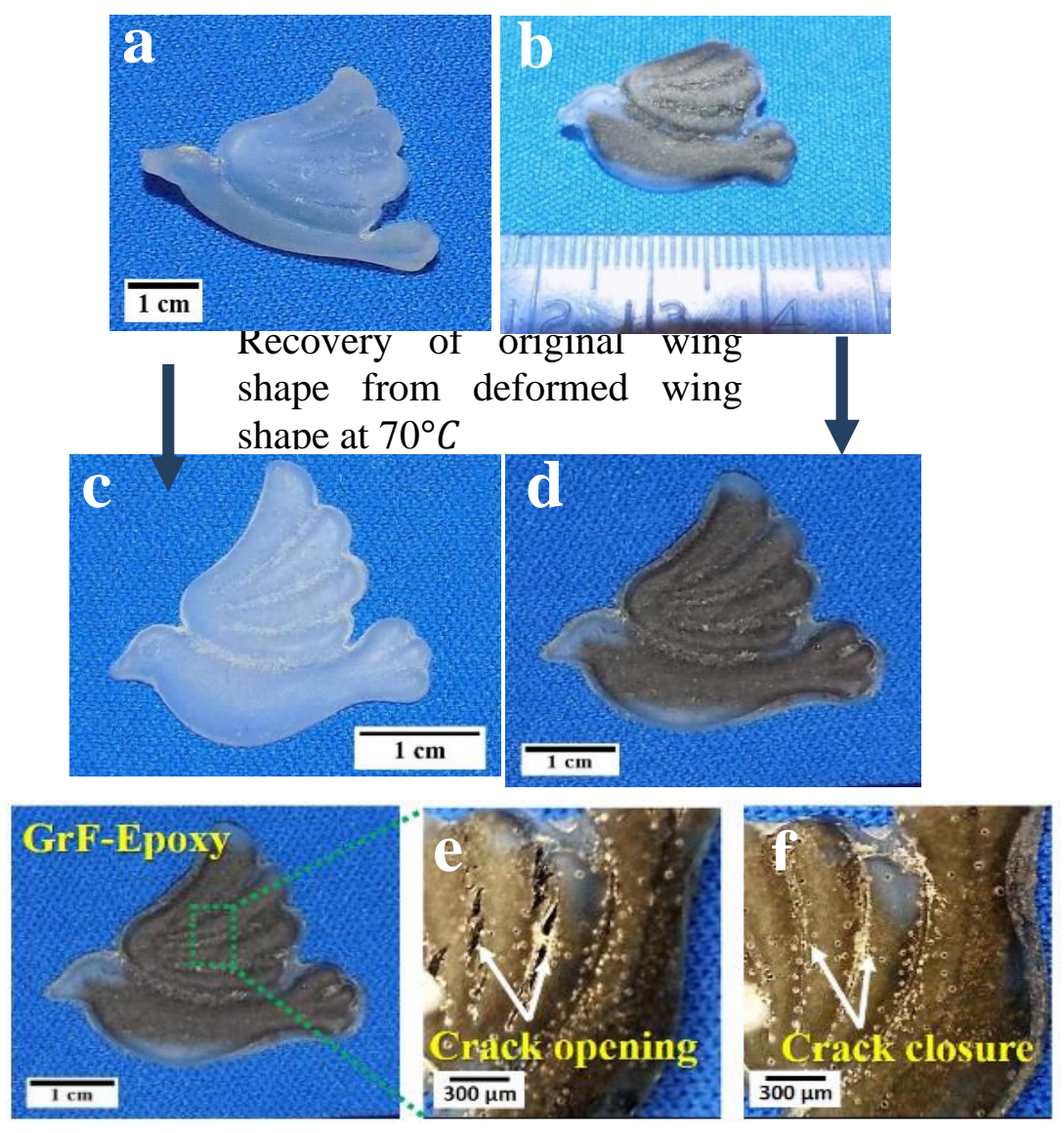

Figure 46: (a) Images of deformed (temporary shape) wings of SMP epoxy-GrF bird and (b) SMP epoxy-GrF bird at $25^{\circ} \mathrm{C}$; (c) Images of recovery of the deformed wings of SMP epoxy bird and (d) SMP epoxy-GrF SMP bird (e) Image of cracked-open wing of biomimetic SMP epoxy-GrF bird after $15^{\text {th }} \mathrm{T}-\mathrm{M}$ cyclic deformation.; (f) Images of the crack-closed wing of SMP epoxy-GrF bird 


\section{Chapter V: Mechanical Behavior of SMP Epoxy-GrF Composite}

It has been established in the preceding section that SMP epoxy-GrF composite exhibit shape memory effect when triggered by thermal and electrical stimuli. The mechanical properties of the composite are a pivotal factor for it to exhibit a shape memory effect. SMP epoxy matrix is selected as a matrix for this study primarily due to its robust aliphatic and aromatic epoxides, and low curing shrinkage [16]. This chapter describes the static and dynamic mechanical properties of SMP composites after GrF addition.

Static mechanical properties of SMP epoxy-GrF composite include ultimate tensile strength, elastic modulus, and fracture strain. The composite dynamic mechanical properties primarily consist of storage modulus, loss modulus, and loss tangent. These properties could provide further insight into additional mechanical behaviors such as shear modulus, recovery force, etc. The knowledge of the static and dynamic mechanical properties would help in the prediction of structural behavior, which would ensure the safety and accuracy control of engineering components.

\subsection{Uniaxial Tensile Properties}

Uniaxial tensile properties can be identified as a form of static mechanical properties of a material, derived from its stress-strain behavior. The stress-strain curves of mold-cast SMP epoxy-based samples were calculated and derived from the measured forcedisplacement curves, as highlighted in the materials and methods section (equations 3.2 and 3.3). The stress-strain curves and Young modulus of SMP epoxy with and without GrF reinforcement are shown in figure $47 \mathrm{a}$ and $\mathrm{b}$. The corresponding mechanical properties deduced from tensile tests are listed in Table 5.1. 

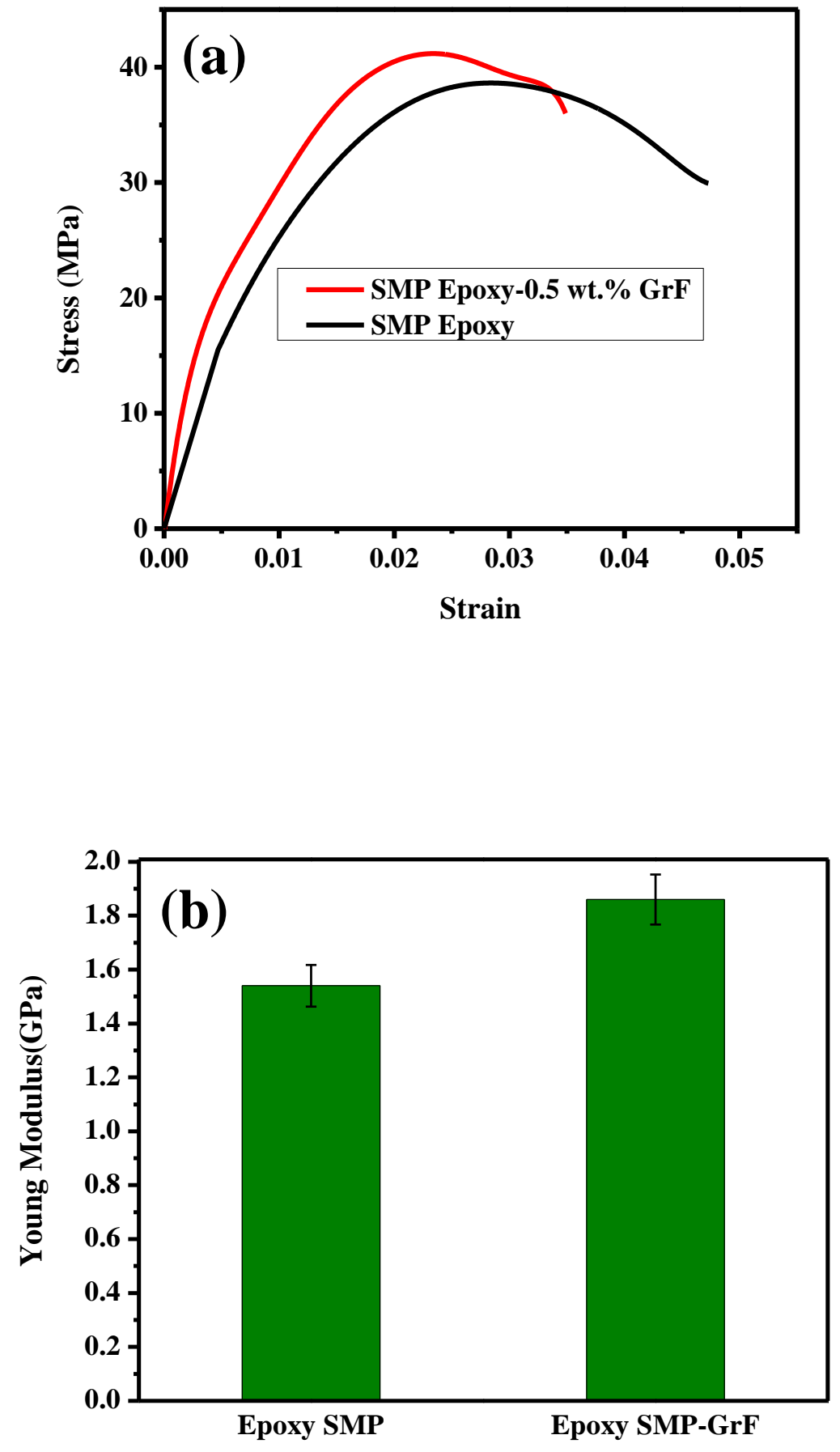

Figure 47: (a) Uniaxial tensile stress-strain curves for SMP epoxy and mold cast composite of 0.5 wt.\% GrF content (b) Comparison of Young modulus (elastic modulus) of SMP epoxy and composite of $0.5 \mathrm{wt} . \% \mathrm{GrF}$ content. 
Table 5.1. Tensile Properties of SMP Epoxy and SMP Epoxy-0.5 wt.\% GrF Composite

\begin{tabular}{|l|c|c|c|c|}
\hline Sample & $\begin{array}{c}\text { Elastic Modulus } \\
(\mathbf{G P a})\end{array}$ & $\begin{array}{c}\text { Ultimate Tensile } \\
\text { Strength (MPa) }\end{array}$ & $\begin{array}{c}\text { Strain at } \\
\text { failure (\%) }\end{array}$ & $\begin{array}{c}\text { Modulus of } \\
\text { Toughness } \\
\left(\mathbf{M J} / \mathbf{m}^{\mathbf{3}}\right)\end{array}$ \\
\hline SMP Epoxy & $1.543 \pm 0.077$ & $37.87 \pm 1.89$ & 4.8 & 1.22 \\
\hline SMP Epoxy-GrF & $1.860 \pm 0.093$ & $42.05 \pm 2.10$ & 3.6 & 1.84 \\
\hline
\end{tabular}

It is noticed that elastic modulus of SMP epoxy-GrF nanocomposite is enhanced by $20 \%$, as compared to SMP epoxy sample. The nanocomposite also experienced $6 \%$ improvement in ultimate tensile strength (UTS) of the nanocomposite than that of SMP epoxy. The composite ductility was, however, restricted (failure strain of 3.6\%) as compared to SMP epoxy (failure strain of 4.7\%). The high in-plane elastic modulus of graphene sheets though accounts for limited failure strain displayed by the composite; GrF node-branch configuration enables it to induce strengthening mechanisms that allow stress transfer from matrix to the reinforcement. Also, the area under stress-strain curve is a measure of fracture toughness of SMP epoxy and SMP epoxy-GrF composites.

Area under the curve $=\mathrm{U}_{\mathrm{T}}=\int_{0}^{\epsilon_{\mathrm{f}}} \sigma \mathrm{d} \epsilon$

An approximation of the modulus of toughness $\left(\mathrm{U}_{\mathrm{T}}\right)$ is obtained by dividing the stressstrain curve into triangular section and rectangular section. A 50\% improvement in toughness behavior of SMP epoxy-GrF composite is attributed to mechanical resistance offered by the network of graphene foam. Figures $48 \mathrm{a}-\mathrm{d}$ reveal the fracture surface of the SMP epoxy-GrF composite. 

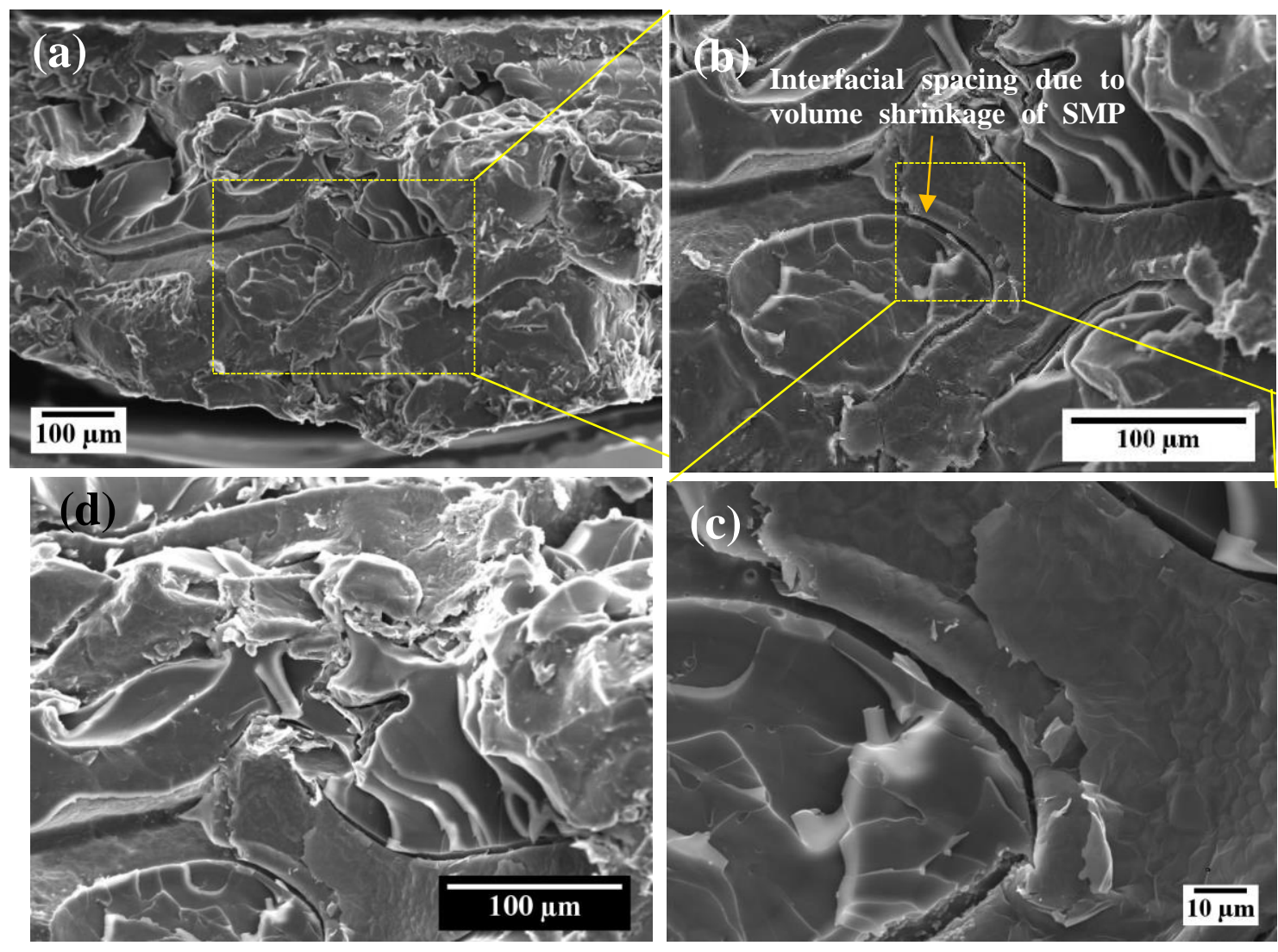

Figure 48: SEM images of the fracture surface of SMP epoxy-GrF composite. (a) GrF branch-node anatomy retaining its integrity in SMP epoxy after curing (b) shrinkage of SMP epoxy leading to the interfacial spacing between GrF and SMP epoxy (c) distinct surface features of GrF and SMP epoxy showing higher magnification of the interfacial spacing (d) close view of good interface regions between GrF and SMP epoxy.

Figure 48a clearly shows that GrF has robust structural integrity because it retained is branch-node anatomy after tensile fracture of SMP epoxy composite. Remarkable in-plane stiffness of graphene sheet can be attributed to its preserved frame-work [24]. This attribute supports the strengthening mechanism during the tensile deformation of the composite. Further magnifications as shown figures $48 \mathrm{~b}$ and $\mathrm{c}$ reveal interfacial spacing region $(\sim 2 \mu \mathrm{m})$ between SMP epoxy and GrF. This is due to shrinkage of the polymer during cthe uring process. The spacing, however, did not limit GrF to reinforce the matrix stiffness. 
This is because GrF reinforcement has la arge surface area which enables more interaction with SMP epoxy chain in other regions of the composite (figure 48d). Figure 48 thus portrays that despite the interfacial spacing between the two components, the composite still exhibits higher tensile strength than the matrix. This suggests that SMP epoxy-GrF composite has great potential to improve the tensile strength if interfacial spacing is significantly minimized. The tensile deformation behavior of SMP epoxy-GrF can also be explained using thermodynamics concept as explained below.

\subsubsection{Thermodynamic Response of Mechanical Behavior of SMP epoxy-GrF}

The amount of stretching demonstrated by a tensile specimen for most materials, when subjected to the small applied load, is influenced by the stiffness of the chemical bonds or enthalpic response at the atomic level. Nevertheless, the stiffness of some materials - particularly rubber, polymer or plastic - stems not from bond stiffness. It arises from entropic factors [152]. This construes that an ideal polymer, under tensile force, is the one that gives a complete entropic response with negligible internal energy changes. Thus, polymers with coiled conformation have high entropy or many possible microstates. Polymers chains with extended conformation have low entropy or very few microstates. Schematics of polymer chains at high entropy state (before stretching) and low entropy state (after stretching) are shown in figure 49a and b, respectively. 

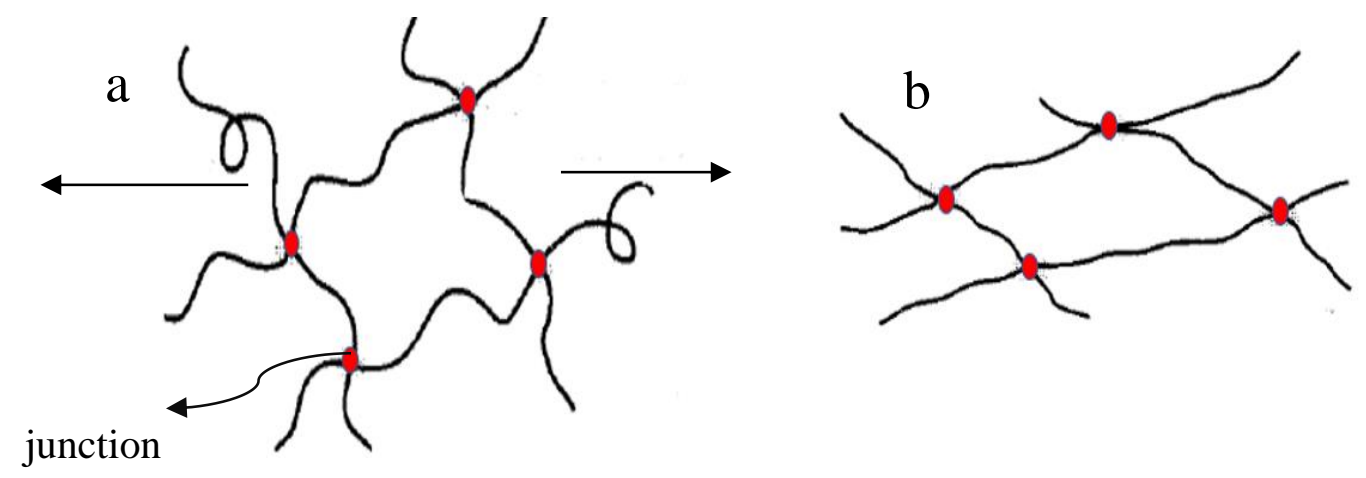

Figure 49: Schematics showing a) Stretching of entangled polymers chains at high entropy state; b) stretched, aligned polymer chains at low entropy state [152]

Deformation of SMP epoxy chains during tensile loading (figure 47a) can thus be interpreted in terms of entropy concept. The entropic response of the polymer chains is theoretically analyzed using Boltzmann equation and strain data from tensile deformation of the samples. The entropy of mixing of the three epoxy resins component is first calculated from the Boltzmann equation used in statistical thermodynamics;

$$
\mathrm{S}=k \ln \Omega
$$

where $k$ is Boltzmann constant $=1.38 \times 10^{-23}$

Based on equation 4.4, the entropy of mixing for the epoxy resins is;

$$
\Delta S^{\mathrm{mix}}=-k\left[n_{a} \ln n_{a}+n_{b} \ln n_{b}+n_{c} \ln n_{c}\right]
$$

where $n_{a}, n_{b}$, and $n_{c}$ are the mole fractions of resin component $a$ (EPON 826), $b$

(NGDE), and $c$ (Jeffamine D230).

Mole fractions $\left(n_{a}, n_{b}\right.$, and $\left.n_{c}\right)$ are 0.38889 moles. 0.3914 moles, 0.2197 moles. The calculated $\Delta S^{\mathrm{mix}}$ obtained from equation 4.4 is $1.5097 \times 10^{-23} \mathrm{~J} / \mathrm{K}$. The positive entropy value suggests that the initial state of SMP epoxy polymer chains when mixed before 
curing and deformation is in a highly randomly coiled position as shown in the schematic (figure 50).

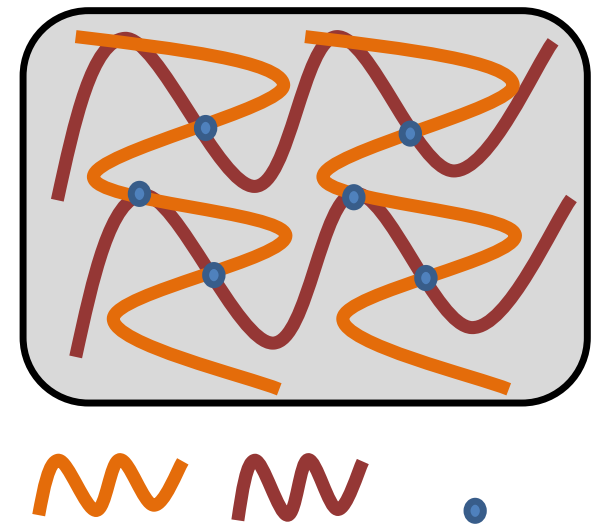

\section{Epon 826 NGDE Jeffamine D230}

Figure 50: Schematic portraying the highly randomly coiled position of SMP epoxy after mixing the three epoxy resins component without curing and chain deformation.

Furthermore, the entropic effect on the deformation of the polymer chains after curing can be determined from the insights provided by Helmholtz free energy as indicated in equation 4.6;

$$
\mathrm{A}=\mathrm{U}-\mathrm{TS}
$$

This means that mechanical work, $\mathrm{dW}$, done by a force $\mathrm{F}$ acting through a differential displacement $\mathrm{dL}$ will produce an increase in free energy as shown in equation 4.7 and 4.8 ;

$$
\begin{array}{lr}
\mathrm{F} \mathrm{dL}=\mathrm{dW} & \text { eqn } 4.7 \\
\mathrm{dW}=\mathrm{dU}-\mathrm{TdS} & \text { eqn } 4.8
\end{array}
$$

where $\mathrm{U}$ is the internal energy of a system, T is the temperature, and $\mathrm{S}$ is the entropy. To obtain force,

$$
F=\frac{d W}{d L}=\left[\frac{d U}{d L}-T \frac{d S}{d L}\right]
$$


Consider SMP Epoxy as an ideal elastomer (rubbery material), the internal energy change, $\mathrm{dU}$, is negligible; so, the force is related directly to the temperature and the change in entropy dS produced by force. To determine the force-deformation relationship, we need to consider how entropy changes with deformation. And based on statistical thermodynamics, changes in configurational probability are related to corresponding changes in thermodynamic entropy by the Boltzmann relation in equation 4.10;

$$
\Delta S=k \ln \frac{\Omega_{2}}{\Omega_{1}}
$$

where $\Omega_{1}$ and $\Omega_{2}$ represents the configurational probability of

the polymer chain in unpertubed and perturbed states

The relative change in probabilities between the perturbed and unperturbed states can now be written as,

$$
\ln \frac{\Omega_{2}}{\Omega_{1}}=-\frac{1}{2}\left(\lambda_{x}^{2}+\lambda_{y}^{2}+\lambda_{z}^{2}\right)
$$

Since deformation or stretching in SMP epoxy and its composites can be described in terms of extension ratios, which are the ratios of stretched to original dimensions, $\frac{\Delta L}{L_{o}}$, stretches in $x, y$ and $z$ directions are denoted by $\lambda_{x}, \lambda_{y}$, and $\lambda_{z}$ respectively.

$$
\text { Thus, } \Delta S=-\frac{k}{2}\left(\lambda_{x}^{2}+\lambda_{y}^{2}+\lambda_{z}^{2}\right)
$$

It is important to note that SMP epoxy and SMP-epoxy-GrF samples are considered as two separate systems during the deformation. For deformation of SMP epoxy, $\lambda_{x}(0.0315)$, $\lambda_{y}(-0.0106)$, and $\lambda_{z}(-0.01)$ are obtained from strain data used in the stress-strain curves (figure 47a). The strain data were analyzed using direct linear transformation (DLTdv) package in MATLAB platform. Basically, tensile test videos of the samples are loaded into the DLT package, where the sample displacements are evaluated from pixel 
movthe ement of the tensile test video. The package analyzes the pixel movement and converts it to strain data. Imputing these values in equation (4.12) gives $-8.31 \times 10^{-27}$ $\mathrm{J} / \mathrm{K}$ as the entropy change $(\Delta S)$ of SMP epoxy after deformation. Stretching or deformation of SMP epoxy-GrF composite can be also computed in the same manner. Values obtained for the extension ratios for the composite are $\lambda_{x}(0.02302)$, $\lambda_{y}(-0.01508)$, and $\lambda_{z}(-0.01)$. The computed result indicated that entropy change $(\Delta S)$ of the composite is $-6.02 \times 10^{-27} \mathrm{~J} / \mathrm{K}$. Negative entropy values of SMP epoxy and SMP epoxy-GrF samples confirm that stretching decreases the entropy of the polymer chains compared to when unstretched (figure 51)
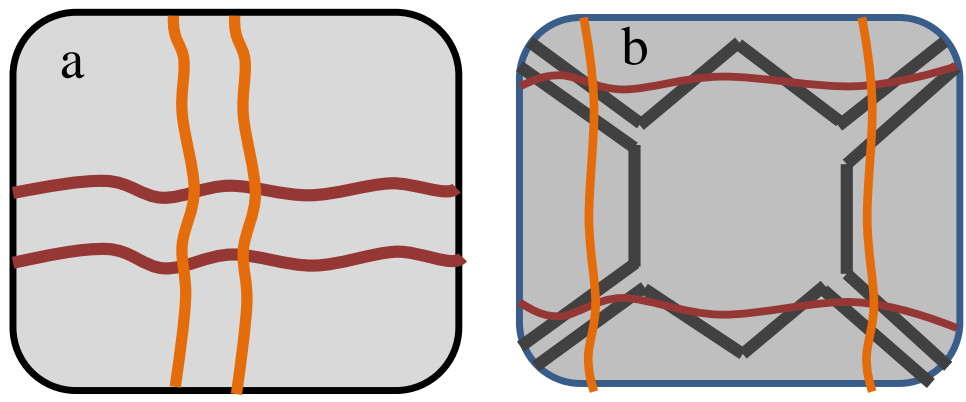

Figure 51: Schematic portraying (a) highly stretched SMP epoxy chain and (b) SMP epoxy-GrF samples after curing and deformation.

Furthermore, it is observed that the entropy value of the composite system is slightly more positive that SMP epoxy. This suggests that GrF addition in thermoset SMP epoxy causes more chain disorder. Node-branch configuration of GrF along the pathway of stretched polymer chains could result in randomly coil chains within individual GrF pores and around its branches.

The thermodynamic analysis has fundamentally explained the behavior of SMP epoxy molecular chains during tensile deformation. In order words, the more randomly 
coiled chains in the composite system (higher entropy than the matrix), the less stretching of the chains and vice-versa. It thus attests that the stiffness behavior of SMP epoxy and composite largely stems from the entropic response of the molecular chains.

\subsection{Dynamic Mechanical Analysis}

Dynamic mechanical analysis (DMA) is a technique that reveals the change of molecular motion in viscoelastic materials (polymers and SMPs) when influenced by frequency and temperature. It is a straightforward method to obtain viscoelastic properties (storage modulus, loss modulus, and tan delta), which contain the in-phase and out-ofphase components. The storage modulus of SMP epoxy is the in-phase component, representing the elastic portion. It measures the ability of the polymer to store energy during deformation. The polymer loss modulus is the out-of-phase component, signifying the viscous portion. It measures energy dissipated as heat. The relationship between these components and phase lag $\delta$ (which is the angle at which the strain $\left(\varepsilon_{0}\right)$ lags the stress $\left(\sigma_{o}\right)$ are given by;

$$
\begin{aligned}
\sigma^{\prime} & =\sigma_{o} \cos \delta \\
\sigma^{\prime \prime} & =\sigma_{o} \sin \delta
\end{aligned}
$$

where $\sigma^{\prime}$ and $\sigma^{\prime \prime}$ are in-phase and out-of-phase stress components

Dynamic moduli for the components can be written

$$
\begin{aligned}
& E^{\prime}=\frac{\sigma^{\prime}}{\varepsilon_{0}}=E^{*} \cos \delta \\
& E^{\prime \prime}=\frac{\sigma^{\prime \prime}}{\varepsilon_{0}}=E^{*} \sin \delta
\end{aligned}
$$

The angle $\delta$ allows characterizing the dynamic moduli in terms of complex notation;

$$
E^{*}=E^{\prime}+i E^{\prime \prime}
$$


where $E^{*}$ is the complex modulus and $E^{*}=\frac{\sigma_{O}}{\varepsilon_{0}}$

The ratio of loss modulus to storage modulus yields loss tangent also known as $\tan \delta$, as shown in equation 4.17

$$
\frac{E^{\prime \prime}}{E^{\prime}}=\frac{\left\{\frac{\sigma_{O}}{\varepsilon_{0}}(\sin \delta)\right\}}{\left\{\frac{\sigma_{O}}{\varepsilon_{0}}(\cos \delta)\right\}}=\tan \delta
$$

Higher values of loss tangent $(\tan \delta$ ) point to a higher ratio of viscous to elastic response in the polymer. This construes that $\tan \delta$ value can be used to characterize damping of the material. Dynamic mechanical properties of SMP epoxy and its composites were obtained using Electroforce 3100 and DMA 242 Artemis.

\subsubsection{Loss Tangent Behavior of SMP Epoxy Composite Samples under Tensile Deformation}

High-resolution transducer of the Electroforce 3100, which contains $1000 \mathrm{~g}$ force transducer was used for the DMA analysis. In a tensile deformation mode, the samples were subjected to mulitple cycles (1200-12000) under an amplitude load range (50-5 $\mu \mathrm{m})$, at a temperature range $\left(25-70^{\circ} \mathrm{C}\right)$, and heating rate of $3^{\circ} \mathrm{C} / \mathrm{min}$ to reveal its loss tangent behaviors. Figures 52a-c show loss tangent behavior of SMP epoxy and SMP epoxy-0.5 wt. \% GrF composite as a function of cycles at $25^{\circ} \mathrm{C}$ and function temperatures at low frequency $(30 \mathrm{~Hz})$ and high frequency $(100 \mathrm{~Hz})$, respectively. SMP epoxy samples subjected to multiple cycles $(1200-12000)$ at $25^{\circ} \mathrm{C}$ are shown in Figure 52a. The data shows that loss tangent (i.e. measure of damping) of SMP epoxy samples decrease with an increase in the number of cycles. SMP epoxy-GrF exhibits higher loss tangent than that of the matrix. 
Compared to that of SMP epoxy, SMP epoxy- 0.5 wt.\% GrF composite displays higher loss tangent at 30 and $100 \mathrm{~Hz}$. At $30 \mathrm{~Hz}$, loss tangent peaks occur at $60^{\circ} \mathrm{C}$ for SMP epoxy and $50^{\circ} \mathrm{C}$ for the composite (figure $52 \mathrm{~b}$ ). Meanwhile, loss tangent peak of all the SMP epoxy-based samples at $100 \mathrm{~Hz}$ appears at the same temperature $\left(60^{\circ} \mathrm{C}\right)$. It is observed that the loss tangent peak of the composite at $50^{\circ} \mathrm{C}$ is approximately $12.5 \%$ greater than that of SMP epoxy at $60^{\circ} \mathrm{C}$.

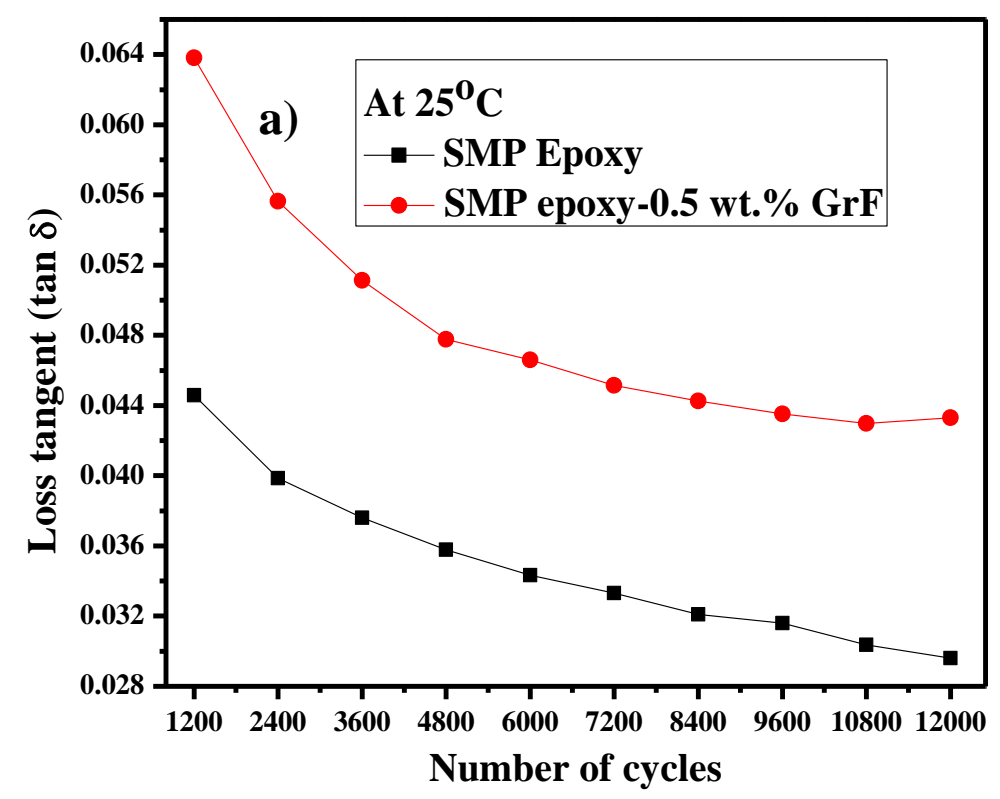



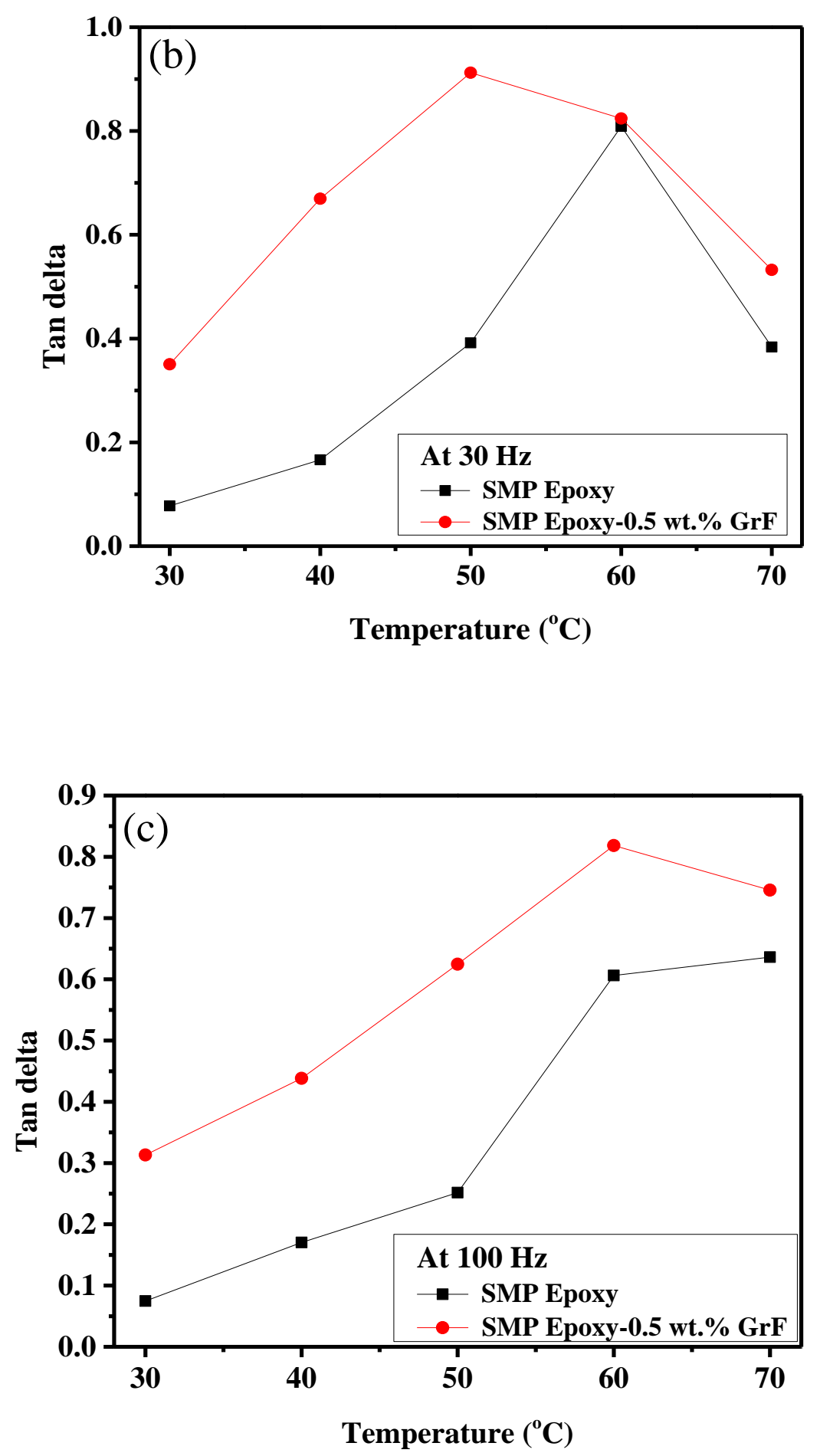

Figure 52: Plot of loss tangent versus of SMP Epoxy and SMP Epoxy-0.5 wt.\% GrF a) as a function of number of cycles; as a function temperature at b) $30 \mathrm{~Hz}$; c) $100 \mathrm{~Hz}$ 
Repeated fatigue cycles as shown in figure 52a could facilitate SMP chain stiffening, explaining the increase the elastic portion of the matrix and composites as the number of cycles increase. Mechanisms such as ripple formation and propagation accounts for the increase in the viscous portion in SMP epoxy-GrF composite. Figures 52b and c show an increase in loss tangents of SMP epoxy-based samples below glass transition $(\mathrm{Tg})$ region, which attain its peaks within the $\mathrm{Tg}$ region. Above the $\mathrm{Tg}$ region, the loss tangents decrease. It construes that as the temperature increases gradually towards the glass transition region (within $45-60^{\circ} \mathrm{C}$ ), molecular frictions between the polymer chains are initiated. This leads to heat dissipation, which contributes to loss tangent increase experienced below $\mathrm{Tg}$. Furthermore, internal structural changes begin to occur in SMP epoxy and its composite. Molecular structure of SMP epoxy-based samples changes from frozen Brownian motion of the polymer chains to mobile segmental chains. Chain mobility activates the viscous portion of SMP epoxy to start gaining momentum ahead of its elastic part, resulting in loss tangent increase of the samples. The cooperative segmental movement of SMP epoxy chains at $\mathrm{Tg}$ region gives rise to loss tangent peak. The decrease in loss tangent above $\mathrm{Tg}$ region is mainly due to decreasing in molecular chain frictions of SMP epoxy as the temperature increases.

It is important to highlight at both 30 , and $100 \mathrm{~Hz}$, SMP epoxy-0.5 wt.\% GrF composite exhibits a higher loss tangent peak than that of the matrix. This portrays the role GrF addition plays in SMP epoxy. It has been established that GrF exhibits excellent damping behavior [70], [86]; thus, GrF reinforcement can be attributed to loss tangent enhancement of SMP epoxy composite. The remarkable GrF behavior stems from the occurrence of a couple of physical mechanisms. Such mechanisms include van deer Waals 
interactive forces between the graphene sheets and formation and propagation of graphene ripples [86]. With the increment in frequency to $100 \mathrm{~Hz}$, loss tangent peak of SMP epoxyGrF composite shifts towards higher temperature (figure 52c) compared to its peak at 30 Hz. This indicates that structural changes are not only dependent on temperature; frequency can also influence chain conformation of the SMP epoxy composite. Higher frequency of $100 \mathrm{~Hz}$ does not allow complete relaxation of polymer chains, thereby gradually restraining molecular motion. It further causes slight stiffness in the chains [153].

Consequently, molecular relaxation can only occur at a higher temperature. This accounts for the shift in the loss tangent peak at $100 \mathrm{~Hz}$. The decrease in loss tangent peak value of the composite from 0.92 to 0.84 also establishes the chain stiffness when the frequency is increased.

\subsubsection{Storage Modulus and Loss Tangent Behavior of SMP Epoxy-GrF under Bending Deformation}

Like Electroforce 3100, DMA 242 E Artemis is also employed to examine the DMA properties of the samples. DMA 242 Artemis is considered for dynamic mechanical characterization of SMP epoxy as it provides 3-point bending deformation mode. Electroforce 3100 does not have this feature. DMA 242 Artemis also has an in-built furnace compartment, unlike Electroforce which requires an external heater unit to subject the material above room temperature. Using DMA 242, the SMP epoxy-based samples are subjected to 3-point bending deformation, amplitude load of 50, 100 and $200 \mu \mathrm{m}$ are applied within the temperature range from $25-100^{\circ} \mathrm{C}$ at hea ating rate of $3 \mathrm{~K} / \mathrm{min}$ and $1 \mathrm{~Hz}$. Figures 53a and $\mathrm{b}$ show storage modulus and loss tangent dependence on temperature and amplitude. 

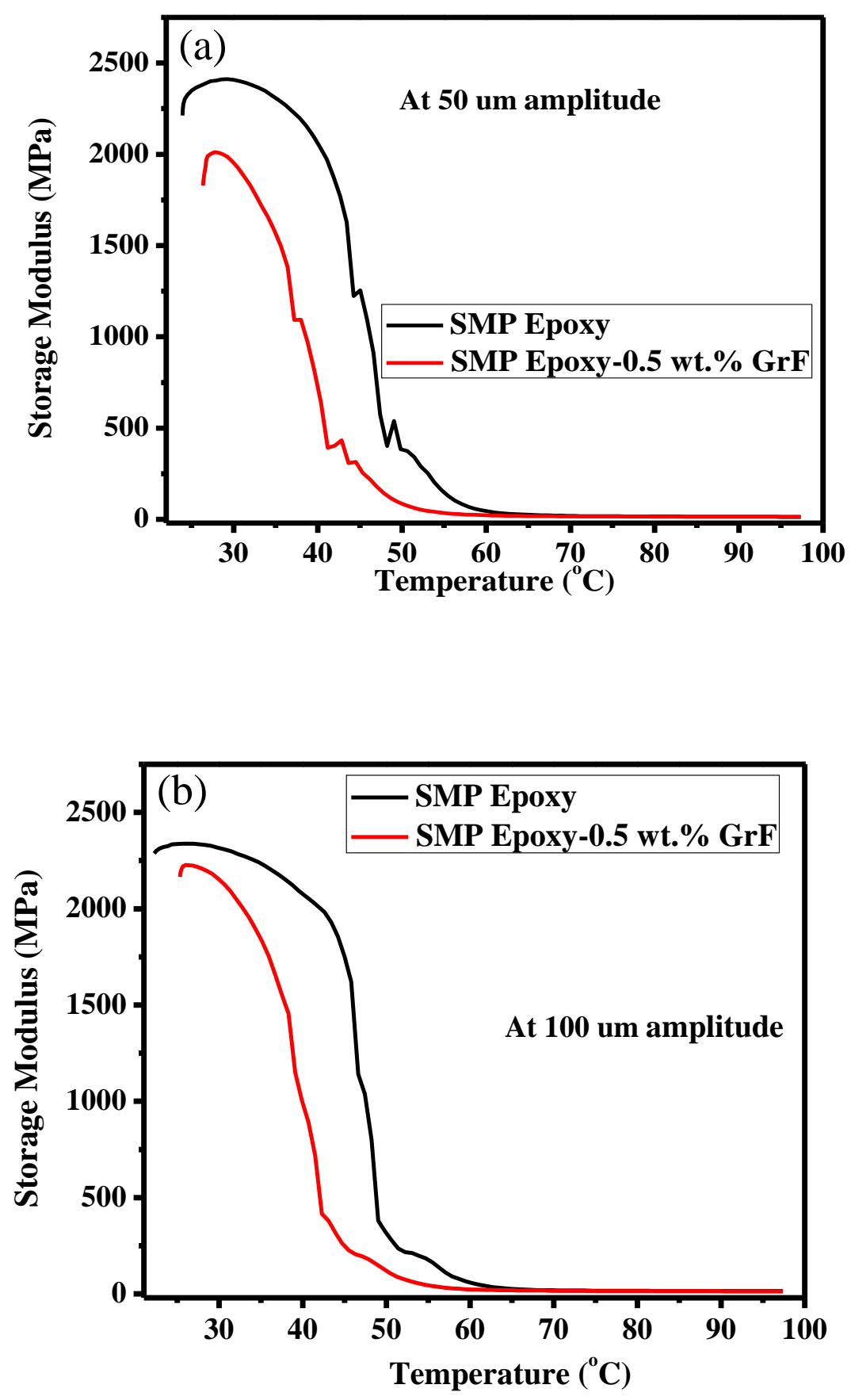


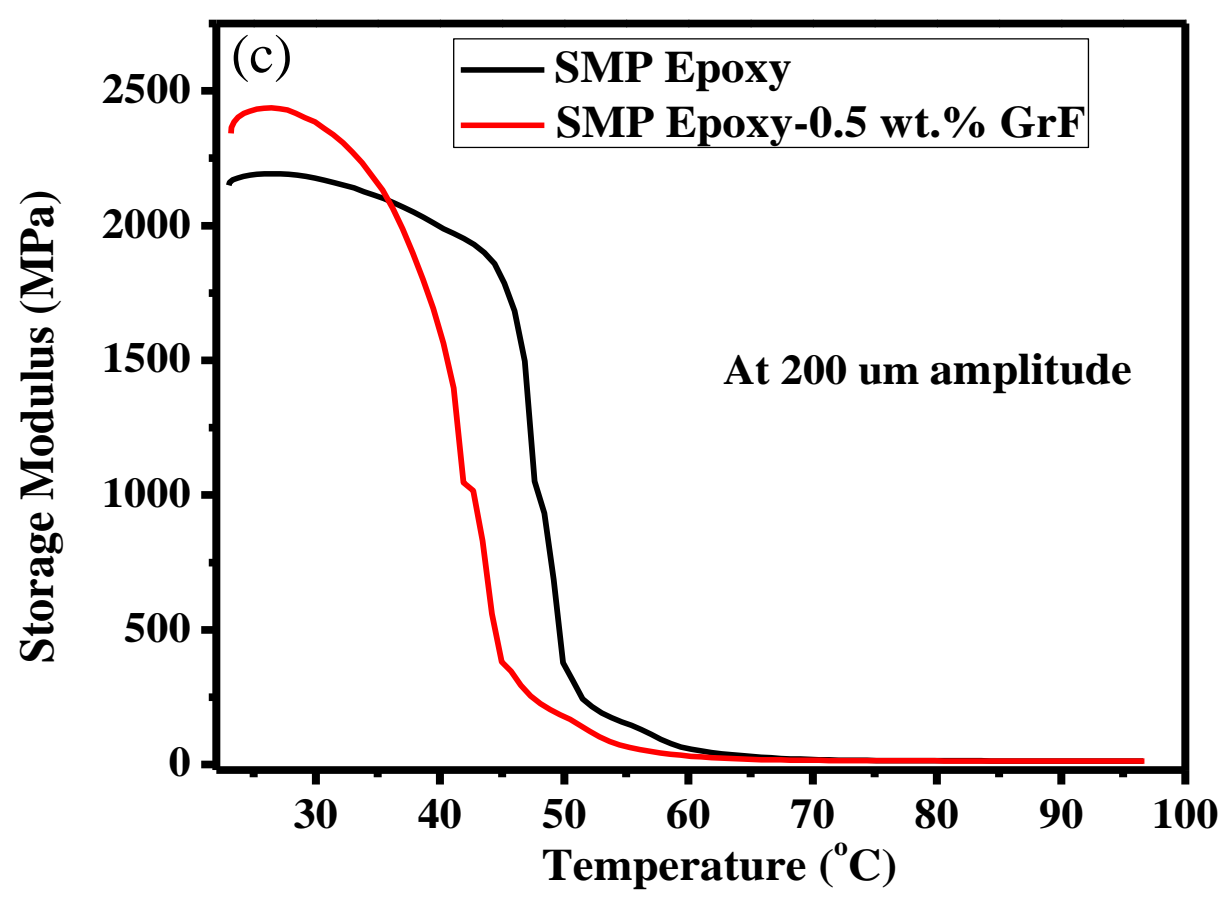

Figure 53: Plot of storage modulus versus temperature of SMP epoxy and SMP epoxy-0.5 wt. $\% \mathrm{GrF}$ at an amplitude of (a) $50 \mu \mathrm{m}$ (b) $100 \mu \mathrm{m}$ and (c) $200 \mu \mathrm{m}$

In figure 53, a decreasing trend in the storage modulus of SMP epoxy with increasing amplitude is observed on the one hand. On the other hand, the SMP epoxy-GrF sample shows an increasing trend in its storage modulus (from 2000 to $2470 \mathrm{MPa}$ ) below $35^{\circ} \mathrm{C}$. At amplitude load of 50 and $100 \mu m$, the storage modulus of SMP epoxy was higher than its composite counterpart at all the given temperatures (figures 53a and b). However, far below the Tg region $\left(25-38^{\circ} \mathrm{C}\right), \mathrm{SMP}$ epoxy-GrF composite shows higher storage modulus than the matrix at amplitude load of $200 \mu \mathrm{m}$ (figure 53c). It is also noticed that above the Tg region (above $60^{\circ} \mathrm{C}$ ), storage moduli of both the SMP epoxy and SMP epoxy-GrF samples appear to be the same value, reducing to almost zero. Figure 54 displays the loss tangent behavior of SMP epoxy-based samples. Like the data trend obtained from 
Electroforce instrumentation technique, SMP epoxy and SMP epoxy-GrF samples show incran ease in loss tangent up to $\mathrm{Tg}$ region. This is subsequently followed by height depression of the loss tangent peak.
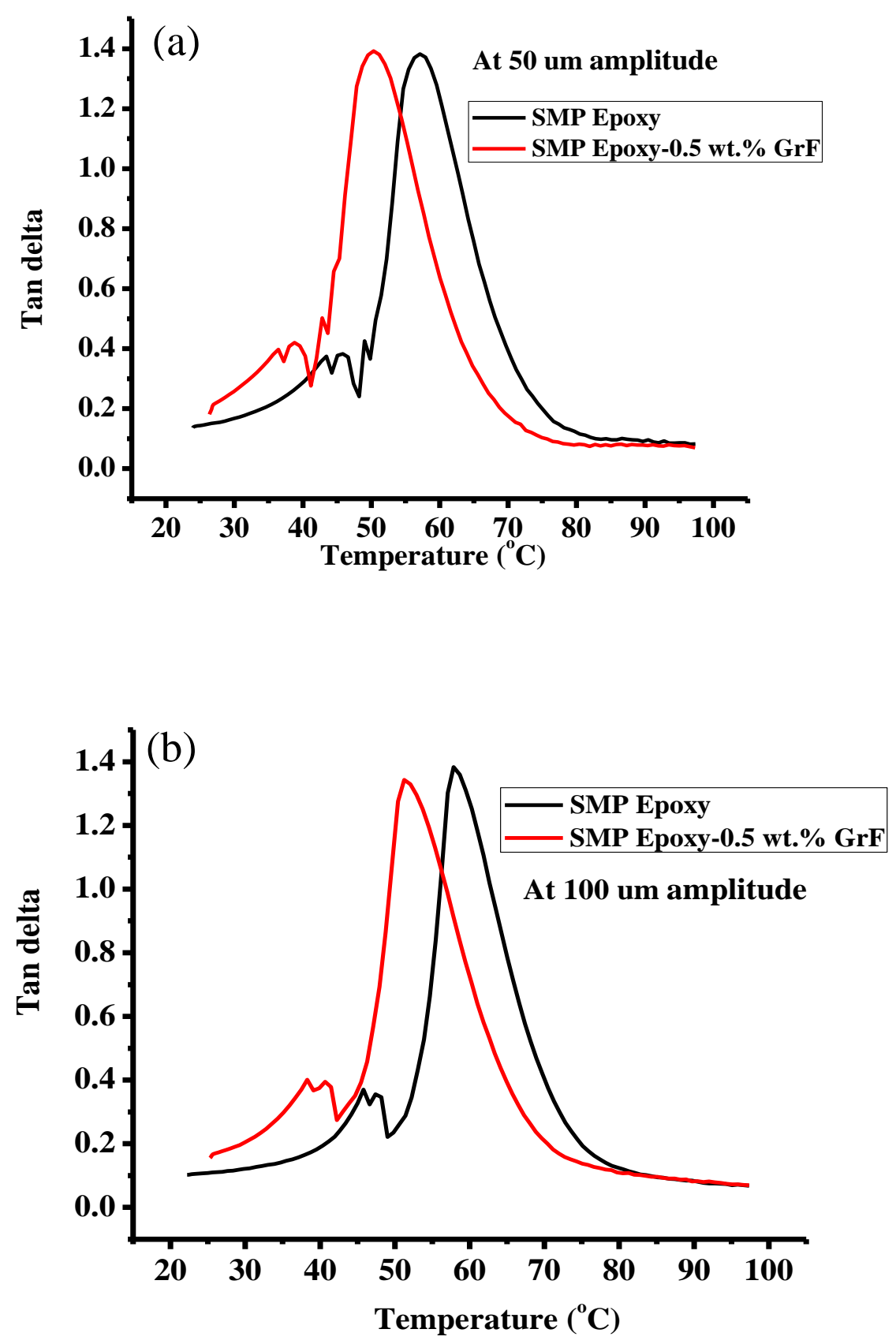

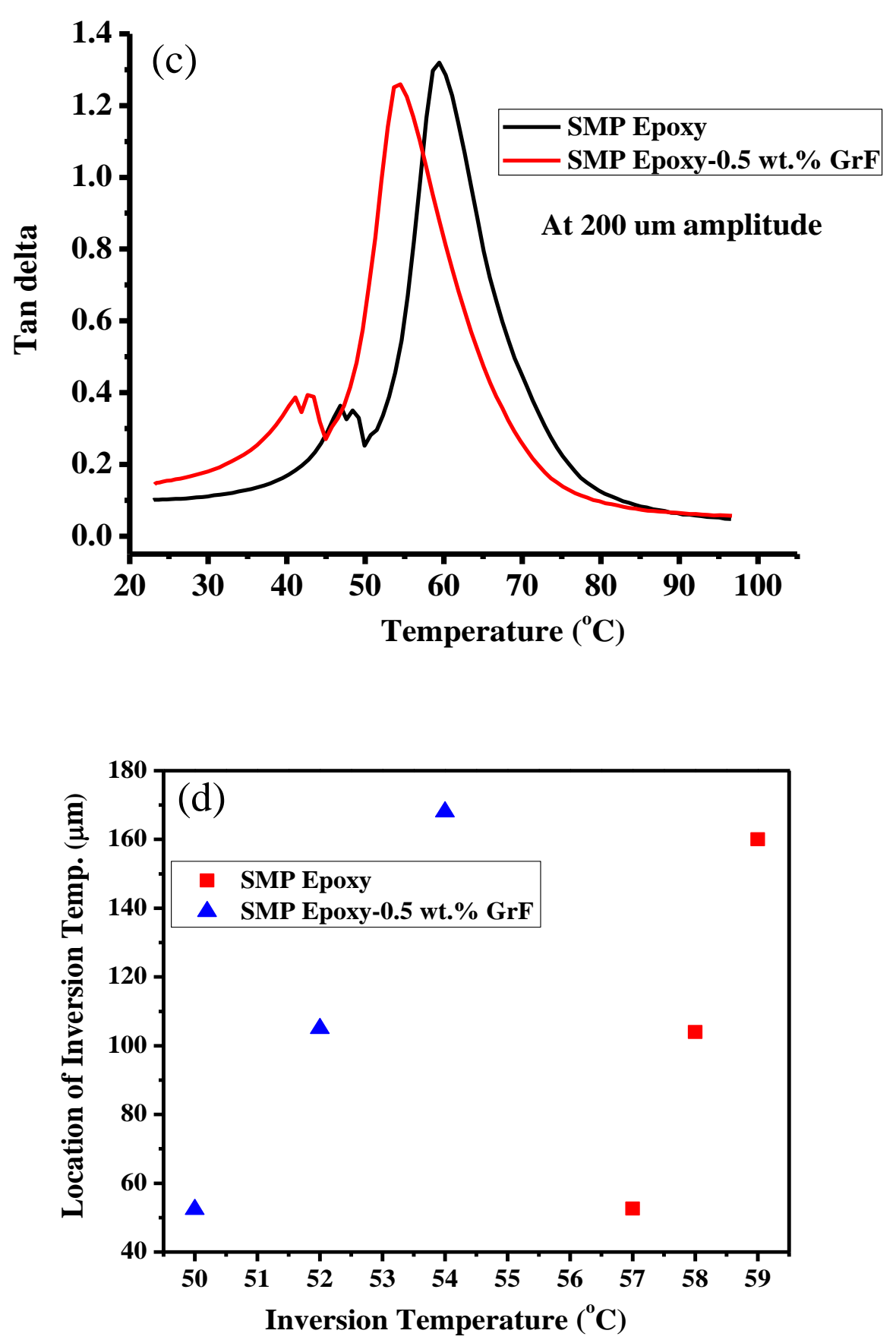

Figure 54: Plot of loss tangent versus temperature of SMP epoxy and SMP epoxy-0.5 wt. $\% \mathrm{GrF}$ at amplitude of (a) $50 \mu \mathrm{m}$ (b) $100 \mu \mathrm{m}$ and (c) $200 \mu \mathrm{m}$; (d) Plot of distance points against inversion temperature of SMP Epoxy and SMP Epoxy- 0.5 wt.\% GrF 
At $50^{\circ} \mathrm{C}$, SMP epoxy-GrF composite demonstrated approximately $180 \%$ increase in loss tangent than SMP epoxy at the three amplitude loads. Above the Tg region, loss tangent gradually assumes the near-zero value. As stated in section 5.2.1, the loss tangent decrease is mainly due to a decrease in energy dissipation due to reduction in frictions between the molecules.

Furthermore, distance points $(\mu \mathrm{m})$ corresponding to loss tangent peak positions of the samples can be extracted from loss tangent curves of the samples (figures 54a-c) after bending deformation using DMA 242 Artemis. These points can be used to observe shifts in the peak position of loss tangent curves of SMP epoxy and its composite samples at the peak temperatures. Figure 54d reveals distance points $(\mu \mathrm{m})$ corresponding to loss tangent peak position as a function of temperature at loss tangent peak. It is observed that as the amplitude load increases from 50 to $200 \mu \mathrm{m}$, the distance points corresponding to the peak position of SMP epoxy and SMP epoxy-GrF composite increases. Compared with that of SMP epoxy, the distance points increase more for the composite (figure 54d).

\subsection{Mechanisms involved in Storage Modulus Behavior}

The displayed results in figure 53 and 54 embody the microscopic response and macroscopic representation of the thermomechanical behavior of SMP epoxy and SMP epoxy-GrF composite. Temperature increase above $25^{\circ} \mathrm{C}$ at $50 \mu \mathrm{m}$ initiates reorganization of the molecular structure of the samples. The reorganization starts with local motion of SMP epoxy chain. As the free volume of the chains increases, larger segments move [154]. The movement corresponds to the bending of side groups and back-bone chain rotation. This results to coordinated large scale chain motions around the $\mathrm{Tg}$ region. In figure 53a 
and b, SMP epoxy-GrF composite shows a slight decrease in storage modulus than that of SMP epoxy at 50 and $100 \mu m$. Physical mechanism such as possible slippage of graphene sheets during polymer chain relaxation could be attributed to this behavior. Few tiny air pockets in between the graphene sheets and SMP epoxy could also contribute to the lower composite storage modulus behavior (figure 53). It is, however, important to note that as the amplitude load increases, the storage modulus of the composite increases below the $\mathrm{Tg}$ region (figure 53a and b). At $200 \mu m$, the composite exhibits higher storage modulus than its counterpart (figure 53c). With the higher amplitude load of $200 \mu \mathrm{m}$, more interaction volume of GrF with SMP epoxy occurs (since the average distance between GrF and epoxy surface is approximately $150 \mu \mathrm{m}$ ). More interaction volume could enable the spring-like effect of GrF. The in-plane stiffness of graphene could also contribute to higher storage modulus behavior from the composite.

\subsection{Mechanisms involved in Loss Tangent Behavior}

It is critical to highlight that two couples of phenomena come into play to explain the loss tangent behavior of SMP epoxy-based samples in figure 54. The first couple is GrF and free volume effects. GrF effect dominates and is accountable for higher loss tangent demonstrated by SMP epoxy-GrF composite than that of the matrix below $\operatorname{Tg}\left(25-58^{\circ} \mathrm{C}\right)$ at the applied amplitude loads. This effect is simply the influence of the energy dissipation capability of GrF. At this temperature range, energy dissipation mechanisms in $\mathrm{GrF}$ are activated. Friction between the SMP epoxy molecules and GrF; van deer Waals opposing forces between the graphene layer and GrF branch flexibility are some of the physical mechanisms that explain higher loss tangent behavior of the composite [86](schematically shown in figure $55 \mathrm{a}$ and $\mathrm{b}$ ). 
Intermolecular friction among the SMP epoxy molecules (arising from covalent bond interaction) and frictional between SMP epoxy chains and graphene sheet (stemming from physical interaction) could contribute to heat dissipation. This, in turn, facilitates dissipation of energy. van deer Waals intermolecular forces between graphene sheets could also increase loss tangent behavior of the composite (figure 55a). The intermolecular opposing forces are mobilized due to compressive forces from 3-point bending, which decrease the graphene sheet interlayer distance. This makes impact energy to absorbed between the layers, thereby promoting loss tangent characteristics of the composite.

Further, the flexural load could cause GrF branch to bend. Branch flexibility also causes energy to be absorbed (figure 55b). These mechanisms enable to SMP epoxy-GrF composite to display higher loss tangent than SMP epoxy at temperature range $\left(25-58^{\circ} \mathrm{C}\right)$ and all applied amplitude loads.
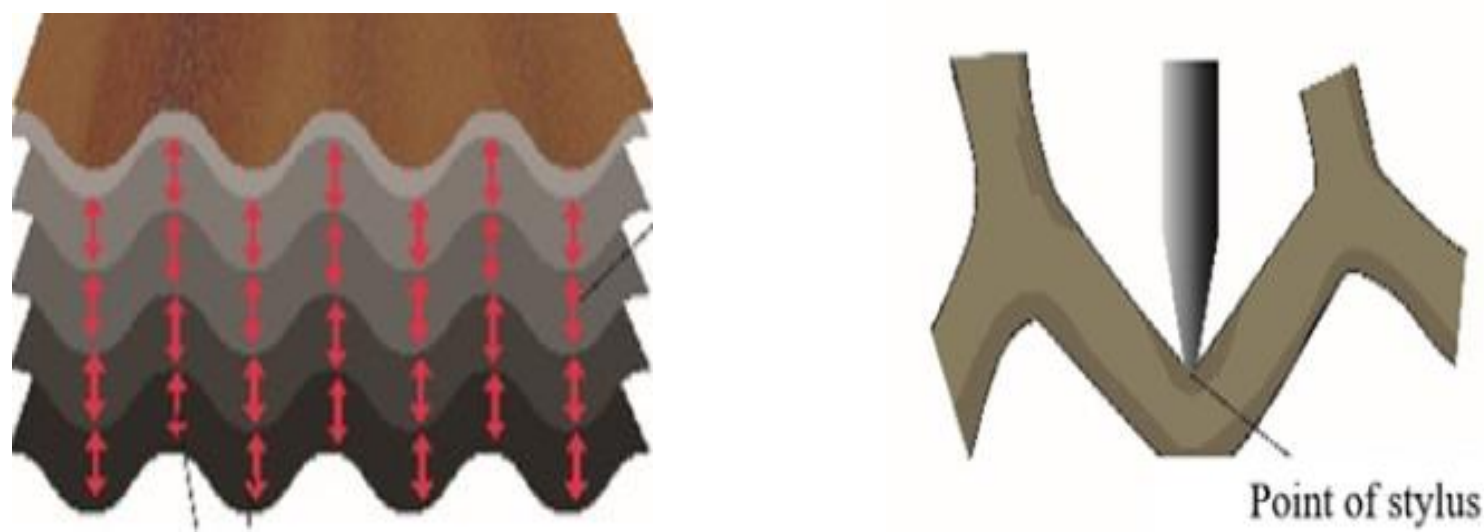

Figure 55: (a) van deer Waals interactions between graphene sheets, and (b) bending of GrF branch during applied load from 3-point bending test [86] 
Above this temperature range, the free volume mechanism plays a role in the loss tangent behavior of the samples. Free volume is the space or gap that is left between SMP polymer chains. GrF filler in SMP epoxy matrix can occupy the spaces, influencing the polymer chain packing. Above $58^{\circ} \mathrm{C}$, molecular resistance of the polymers is overcome, resulting in more chain mobility. However, chain mobility in SMP epoxy-GrF composite is limited due to GrF addition compared with SMP epoxy. Hence, the lower loss tangent of the composite is demonstrated.

The second phenomenon is competing factors of thermal strain and amplitude load in the SMP epoxy-based samples. Thermal strain in terms of different coefficients of thermal expansion (CTE) is first explained. To illustrate, thermal strain investigation on thermal expansivity study is conducted on SMP epoxy and SMP epoxy-GrF samples using a dilatometer. Figure 56 shows the CTE behavior of SMP epoxy-based samples at these temperatures $\left(26-39^{\circ} \mathrm{C}\right)$. SMP epoxy exhibited higher CTE compared with SMP epoxyGrF composite. This indicates that GrF interaction with SMP epoxy has resulted in lower CTE behavior of the composite in comparison to that of the matrix. Since CTE is associated with bonding [155], the CTE results suggest bonding interaction between GrF and SMP epoxy chains. As highlighted from Raman and FTIR results of the SMP epoxy-GrF composites (figure 30a and b), CTE results in figure 56 further corroborates that strong physical (chain entanglements) and weak chemical bonding ( $\mathrm{OH}$ and $\mathrm{NH}$ bonding) exist between GrF and SMP epoxy chain. This thus implies that GrF reinforcement can minimize the thermal expansion of SMP epoxy. 


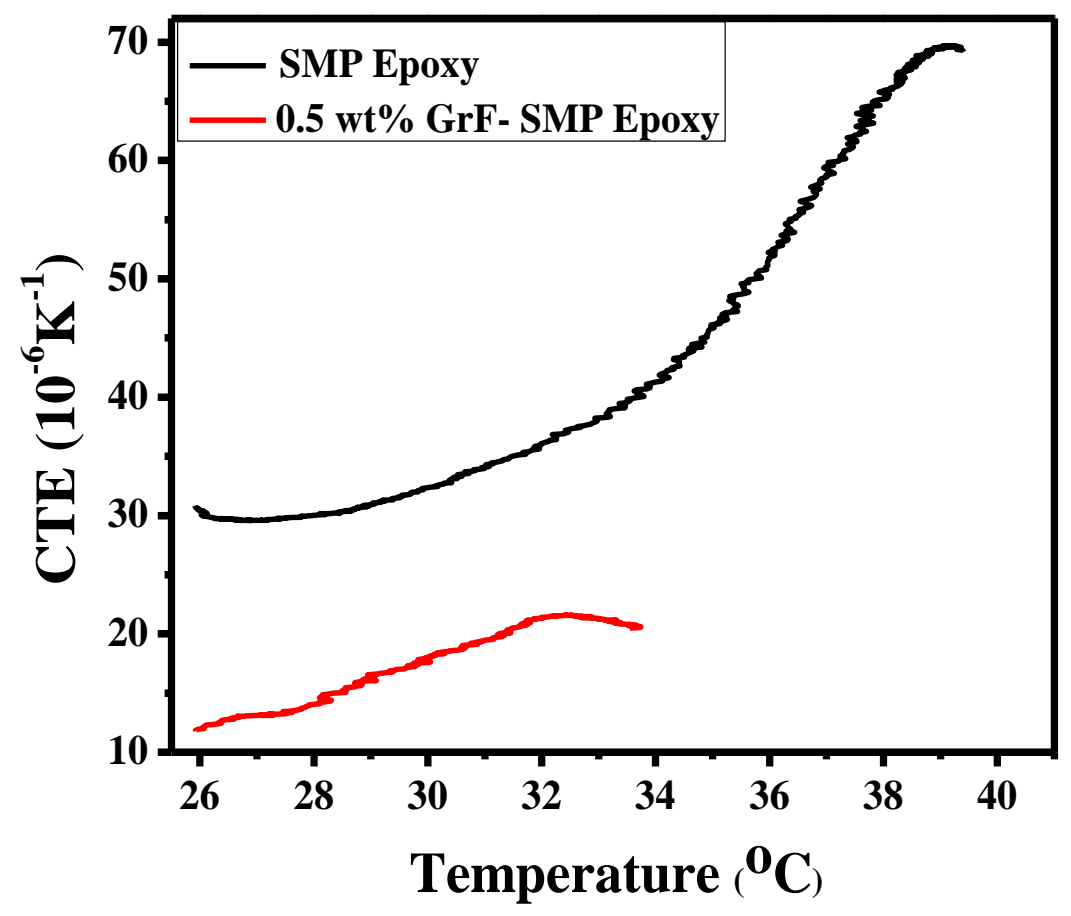

Figure 56: Plot of Coefficient of thermal expansion vs. temperature of SMP epoxy and SMP-0.5 wt.\% GrF Composite

Based on the CTE results in figure 56, investigation of thermal expansion of $\mathrm{GrF}$ reinforcement is considered. Figure 57 shows the CTE results of a unit and multiple cells of GrF. 

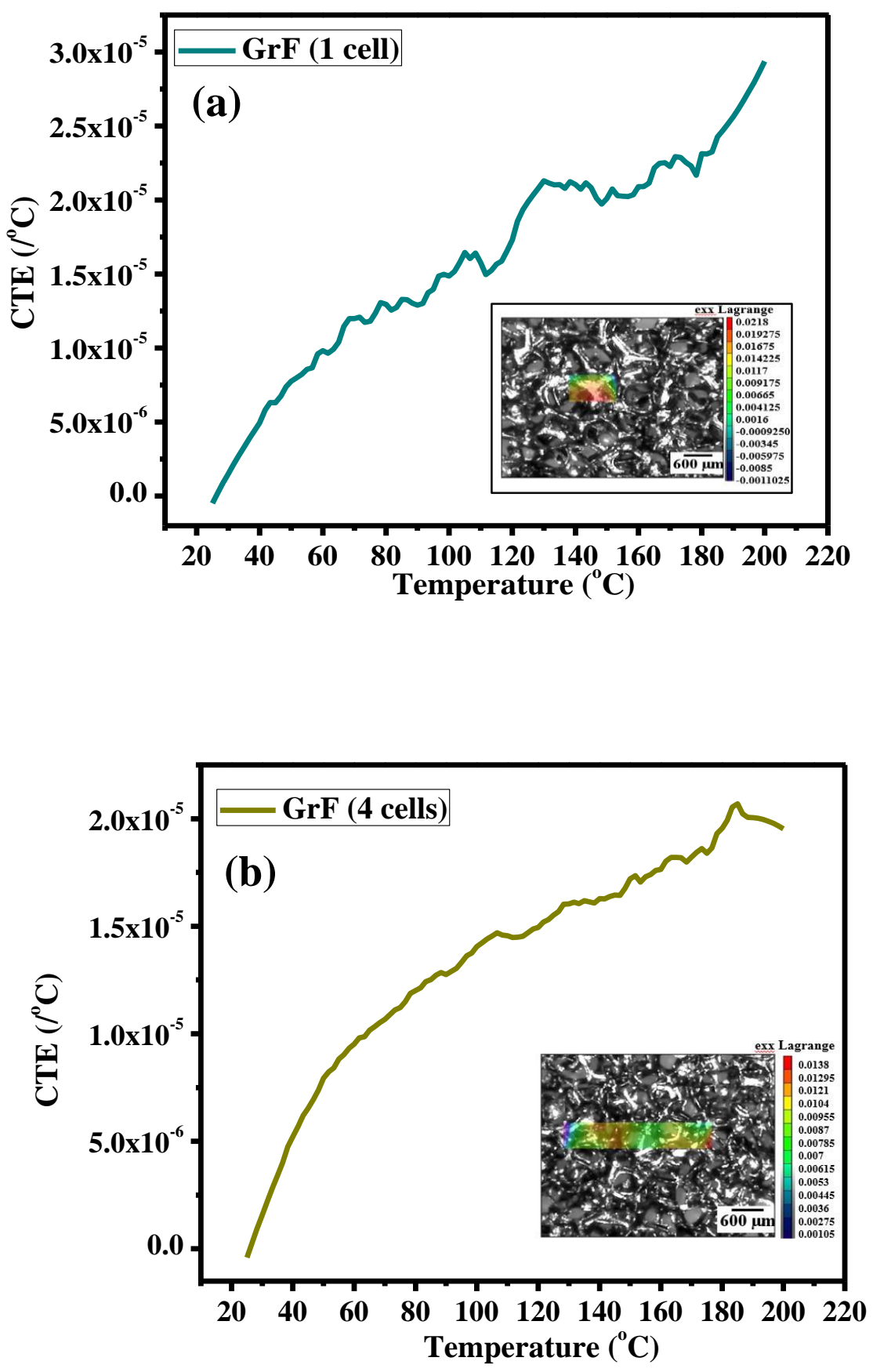


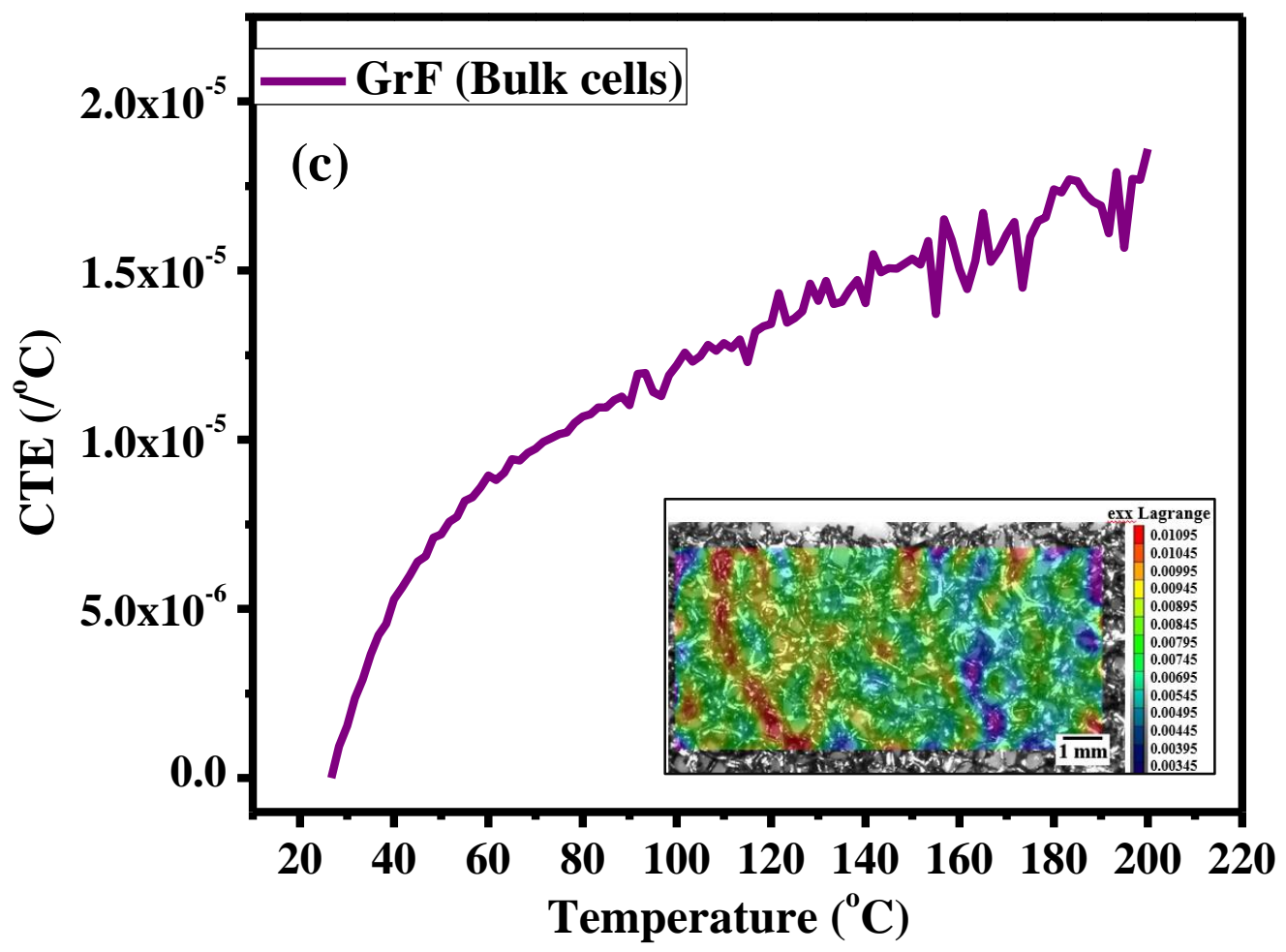

Figure 57: Plot of Coefficient of thermal expansion vs. temperature of GrF (a) 1 cell; (b) 4 cells and (c) Bulk cells

It is observed that as temperature increases from 25 to $200^{\circ} \mathrm{C}, \mathrm{GrF}$ CTE linearly increases for the unit and multiple cells. At $200^{\circ} \mathrm{C}$, CTE of the GrF unit cell, four cells, and bulk cells are $3 \times 10^{-5}, 2 \times 10^{-5}$ and $1 \times 10^{-5} /{ }^{\circ} \mathrm{C}$, respectively. The result depicts that $\mathrm{CTE}$ decreases from unit to bulk cells of GrF. This could be attributed to the presence of air, which has poor thermal conduction, in between the GrF pores. GrF CTE $\left(1 \times 10^{-5} /{ }^{\circ} \mathrm{C}\right)$ result corroborates nearly three times decrease in CTE experienced by CTE of SMP epoxy after GrF addition, as shown in figure 57. CTE mismatch of SMP epoxy and GrF can thus lead to thermal strain in the composite material. 
At low temperature for all amplitude load (50, 100 and $200 \mu \mathrm{m})$, thermal strain effect is not significant. This makes the amplitude load dominate and contribute to the loss tangent behavior of the samples. That is, amplitude load creates a compressive force on the composite, further triggering dissipative mechanisms (graphene sheet ripples, branch bending, sheet sliding). However, as the temperature increases under these amplitude loads, thermal strain due to CTE mismatch becomes considerably active. This thermal strain is in the form of tensile nature (stretching). The stretching could lead to the alignment of some portion of SMP epoxy chains, making that region to have elastic nature. Consequently, this mechanism could contribute to the decrease in loss tangent of the composite than SMP epoxy.

Furthermore, it is essential to briefly consider a slight change in the peak shift of the loss tangent of the SMP epoxy matrix and composite as a function of temperature at loss tangent peaks (figure 54d). Increase in the loss tangent peak position of the composite can be attributed to a combination of multiple factors. It could be due to synergy of $\mathrm{GrF}$ dissipation mechanisms, structural relaxation of the SMP epoxy chain, and amplitude load (which causes more interaction chain volume of the polymer). These factors enable the viscous character of the composite to be active, influencing loss tangent peak position. Additional studies, however, would need to be conducted to fully ascertain the cause of the peak shift of loss tangent curves of SMP epoxy and its composite system.

Besides the detailed knowledge of mechanical properties of SMP epoxy samples by mold cast approach, 3D printing of SMP epoxy-based samples is conducted as another promising fabrication technique. 


\section{Chapter VI: 3D Printing of SMP Epoxy-Graphene Nanoplatelets Powder Composites by Slurry-Based Extrusion}

This chapter provides details about processing, structure and properties of SMP epoxy-graphene composites by 3D printing technique. Three-dimensional (3D) printing, an additive manufacturing process, involves putting a model design into CAD file and translating it into a three-dimensional physical object through layer-by-layer printing fashion. Fabrication of the composites by this approach presents a feasible route to further explore the capabilities of the shape memory epoxies.

Among the types of 3D printing techniques known [156], this study employed the extrusion-based method. In order words, this study presents 3D printing of SMP epoxy by slurry-based extrusion using Print head (figure 58) that is attached to Hyrel printer. Furthermore, SMP epoxy is also reinforced with graphene nanoplatelets (GNP). GNP has shown great ability to serve as an excellent mechanical and thermal reinforcement in polymers and SMP. SMP epoxy-GNP composite is 3D printed by the same method for the first time, and characterization is performed on the samples.

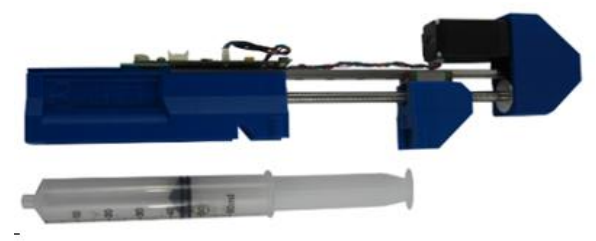

Figure 58: Print head syringe for low viscosity polymers respectively (http://www.hyrel3d.com/core-suystems/system-30m)

\subsection{SMP Epoxy Precursor for 3D Printing: challenge and solution}

It is infeasible to use SMP epoxy in its as-prepared state as 3D printing precursor. As-prepared SMP epoxy is simply a mixture of three liquid resins (two epoxy composition 
and one curing agent). The infeasibility of using the resin mixture as a printing material is due to its viscosity, a fundamental material property. The epoxy mixture, in its liquid state at $25^{\circ} \mathrm{C}$, has a viscosity of $25.6 \mathrm{cP}$. The low viscosity of SMP makes it highly flowable material out of the syringe nozzle during printing. Three different approaches used to address the viscosity issue are further highlighted. However, only one of the approaches comes out successful.

\subsubsection{Mixing of SMP liquid and its powder}

First approach or trial, which was used to increase SMP epoxy viscosity, involves mixing SMP epoxy liquid and powder. SMP epoxy powder is prepared by chopping a cured monolith SMP epoxy to large particles. Liquid nitrogen is added to the particles to make it brittle to be crushed into powdery form. The ball-milling machine is then used to finally process them to fine powders for $20 \mathrm{~min}$. Figure 59a shows SEM micrograph of the SMP epoxy powder where its size is measured. Powder size distribution reveals a particle diameter ranging between 2 to $109.99 \mu \mathrm{m}$ (figure 59b). The distribution indicates that particle size diameter mostly falls within $30-39.99 \mu \mathrm{m}$ from the cumulative distribution. This indicates that the SMP epoxy powder size is far less than the nozzle size; hence, no clogging issue at nozzle tip would exist during 3D printing of the SMP epoxy precursor. 

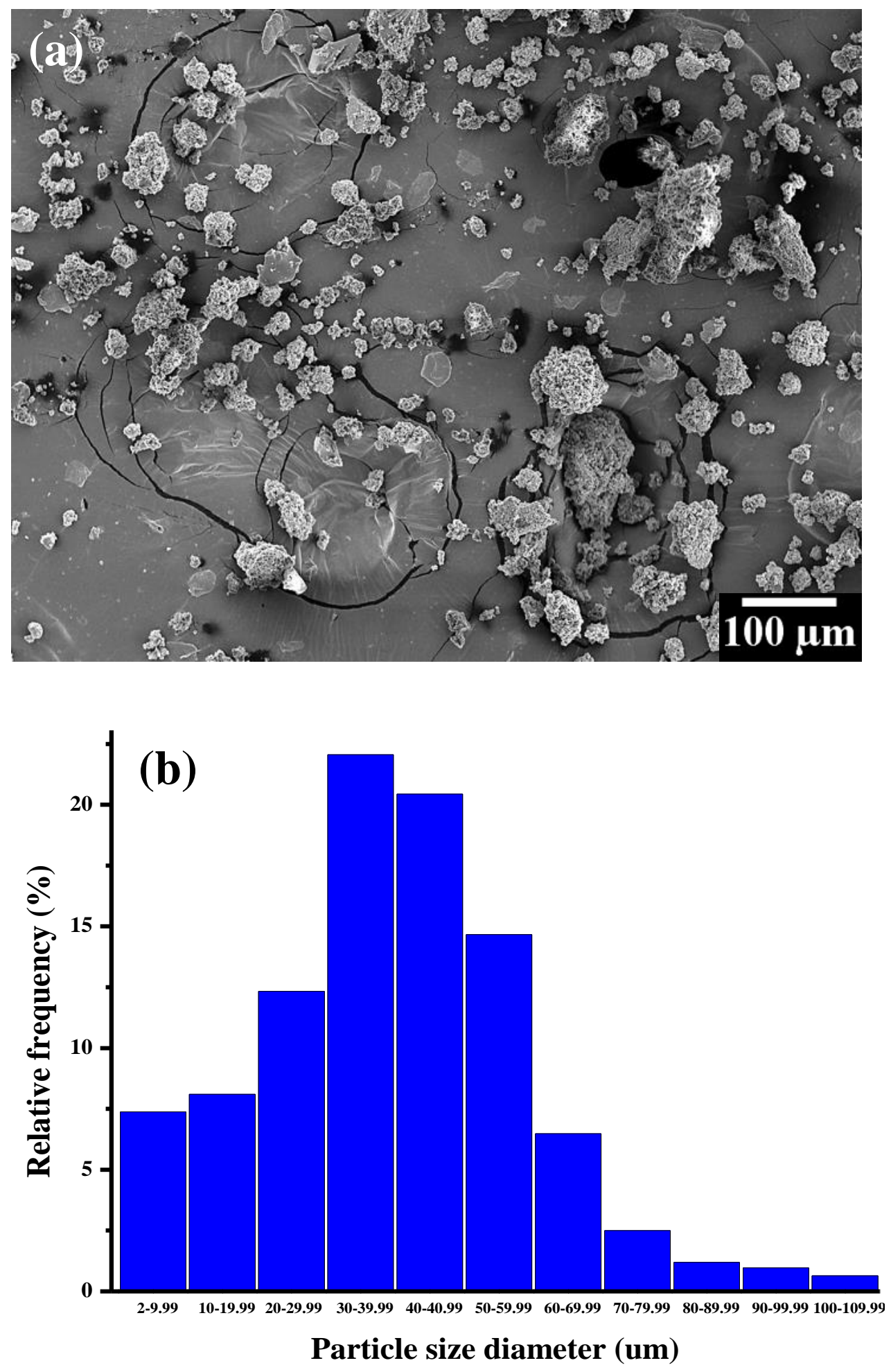

Figure 59: (a) SEM micrograph showing varying sizes of SMP epoxy powder processed by ball milling technique, (b) particle size distribution of SMP epoxy powder. 
It is important to highlight that appropriate viscosity of SMP epoxy paste is required for efficient extrudability of the precursor from the syringe nozzle. Achieving the SMP extrusion with the appropriate nozzle diameter would result in good printing resolution. Thus, the SMP epoxy powder is mixed with its liquid counterpart in the ratio of 3:1 for 30 $\mathrm{s}$ to attain significantly higher viscosity $(\sim 730 \mathrm{cP}$ as comparable with some traditional epoxies in the literature) than that of only SMP liquid $(25.6 \mathrm{cP})$ at $25^{\circ} \mathrm{C}$. It is observed that the mixing of colorless SMP epoxy liquid and white SMP epoxy powder produced a greyish slurry color (figure 60). This could be due to a chemical reaction between the SMP powder (functionalized with liquid nitrogen) and the SMP liquid. The prepared precursor was used for $3 \mathrm{D}$ printing out of syringe nozzle size $(800 \mu \mathrm{m}$, inner diameter $)$, printing velocity of $30 \mathrm{~mm} / \mathrm{sec}$, and a layer height of $200 \mu \mathrm{m}$. More details of the 3D printing are provided in the materials and methods chapter (section 3.2.3).

3D printed dog-bone shape sample obtained from the described precursor smudges almost immediately after printing (figure 60). This construes that the printed sample could not retain the dog-bone shape sample. The smudging noticed after printing is due to shearthinning behavior of the SMP epoxy precursor as it comes out of the nozzle. Consequently, the first approach is not considered for 3D printing of SMP epoxy and SMP epoxy-GNP samples. 


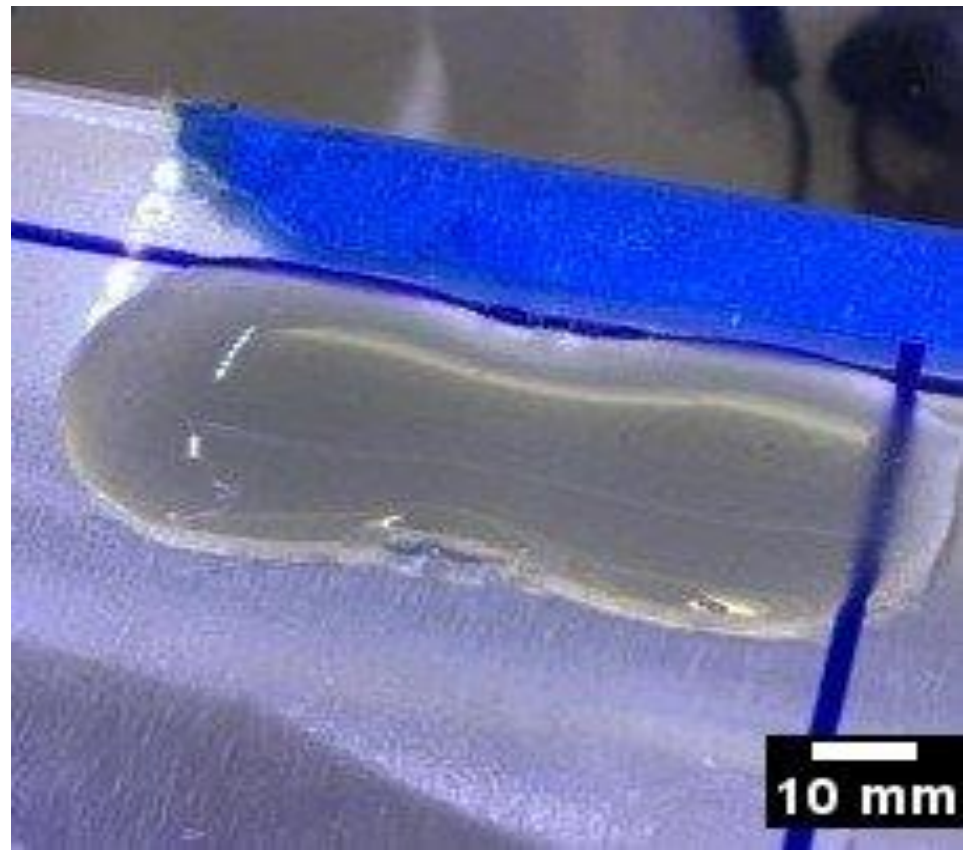

Figure 60: Image showing 3D printed dog-bone shape sample using a mixture of SMP epoxy liquid and powder

\subsubsection{Partial curing of SMP liquid at room temperature}

Partial curing of SMP liquid at room temperature is the second approach considered to overcome the smudging challenge during extrusion-based 3D printing of SMP epoxy. After mixing the resin components, the SMP epoxy liquid is left to partially cure at $25^{\circ} \mathrm{C}$ for 36 hours to achieve a viscosity $(\sim 800 \mathrm{cP})$. The viscosity is slightly higher than the thickness used in the first approach $(\sim 730 \mathrm{cP})$ due to reduced shear-thinning between the SMP epoxy precursors itself and between the SMP epoxy and the walls of the extruder nozzle during printing. Using the same printing conditions for the first approach, the SMP precursor is used to 3D print dog-bone shape sample. The printed sample retained the dogbone shape sample for approximately 30 minutes at room temperature before it started smudging (indicated in figure 61a and b). 

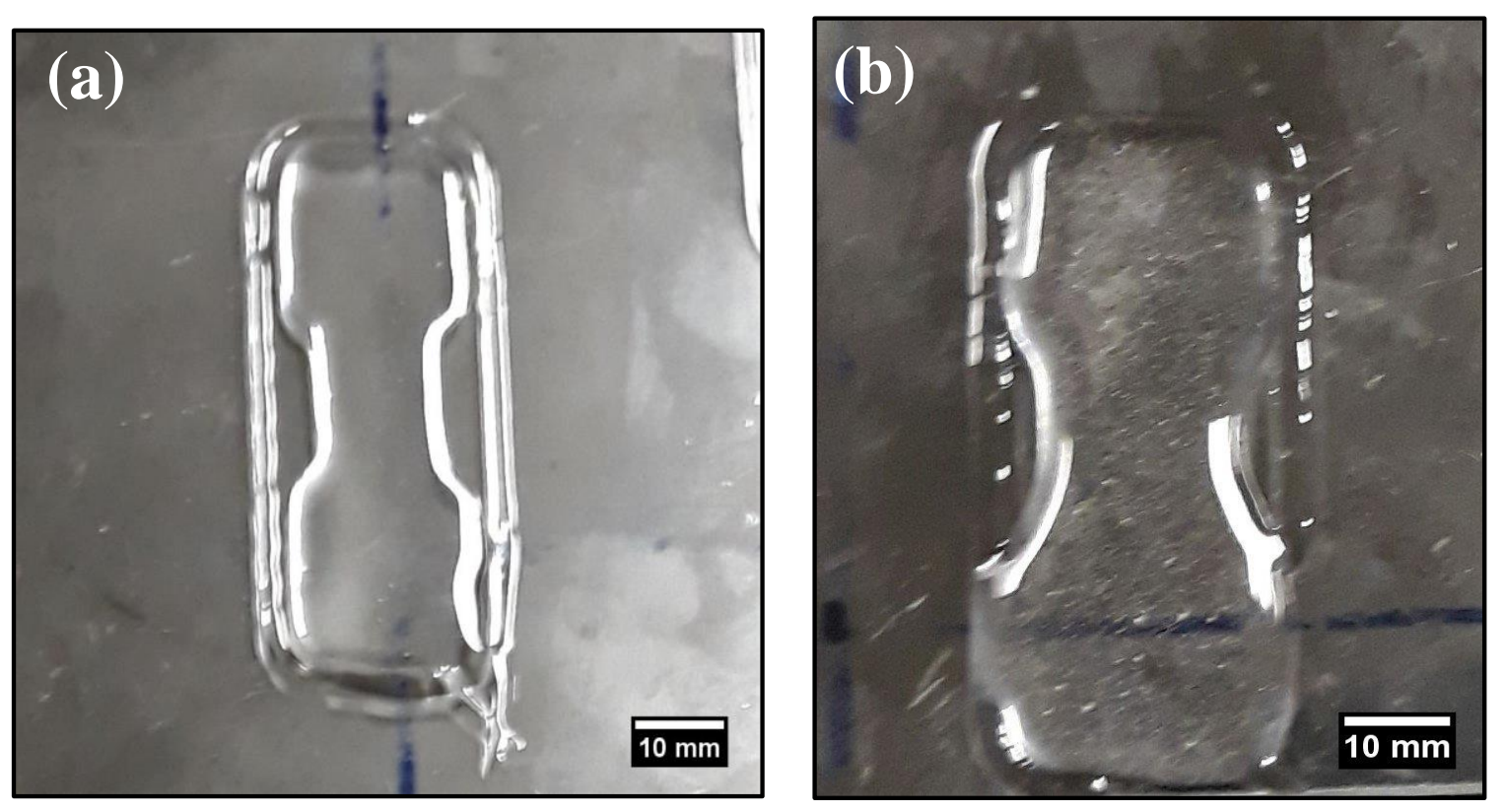

Figure 61: Image of 3D printed dog-bone shape sample using partial cured SMP epoxy precursor. (a) immediately after printing (retaining the dog-bone shape geometry); (b) 30 minutes after printing (revealing smudging of SMP epoxy and not maintaining the shape geometry)

\subsubsection{Partial curing of SMP epoxy liquid at room temperature (placed under a nearly cryogenic condition after $3 D$ printing)}

Like the second approach, the SMP epoxy precursor is prepared by partial curing the SMP epoxy liquid at $25^{\circ} \mathrm{C}$ for 36 hours, maintaining the previous viscosity $(\sim 800 \mathrm{cP})$. However, to overcome shear thinning behavior of the precursor, an additional process is added during and after 3D printing the dog-bone shape sample. The process is in two stages: (i) freeze spraying $\left(10^{\circ} \mathrm{C}\right)$ on the dog-bone shape sample during and after $3 \mathrm{D}$ printing, using freeze spray component consisting of tetrafluropropene, and (ii) subjecting the dog-bone sample to nearly cryogenic condition $\left(-10^{\circ} \mathrm{C}\right)$ for $24 \mathrm{hrs}$ by placing it in a refrigerator.

Insight is drawn from SME phenomenon (discussed in section 4.1) to consider this process. Since shape fixity requires subjecting SMP epoxy far below the $\operatorname{Tg}\left(\sim 20^{\circ} \mathrm{C}\right)$ to 
retain its temporary shape, then this condition is borrowed and used on SMP epoxy precursor for 3D printing. After $24 \mathrm{hrs}$, the 3D printed sample successfully retained the dog-bone geometry after removing it from the refrigerator and left under room temperature to be completely cured. The complete curing took another four days after removal from the refrigerator. This is indicated in figure 62a. Based on the successful extrusion of SMP epoxy samples, the third approach is employed to 3D print SMP-epoxy with $0.1 \mathrm{wt} . \%$ GNP (figure 62b). The viscosity of SMP increases to $12300 \mathrm{cP}$ after $0.1 \mathrm{wt} \%$ GNP addition. 0.1 wt.\% GNP is considered because higher weight percent of GNP can lead to agglomeration and result in nozzle clogging. This could further lead to poor printing resolution.

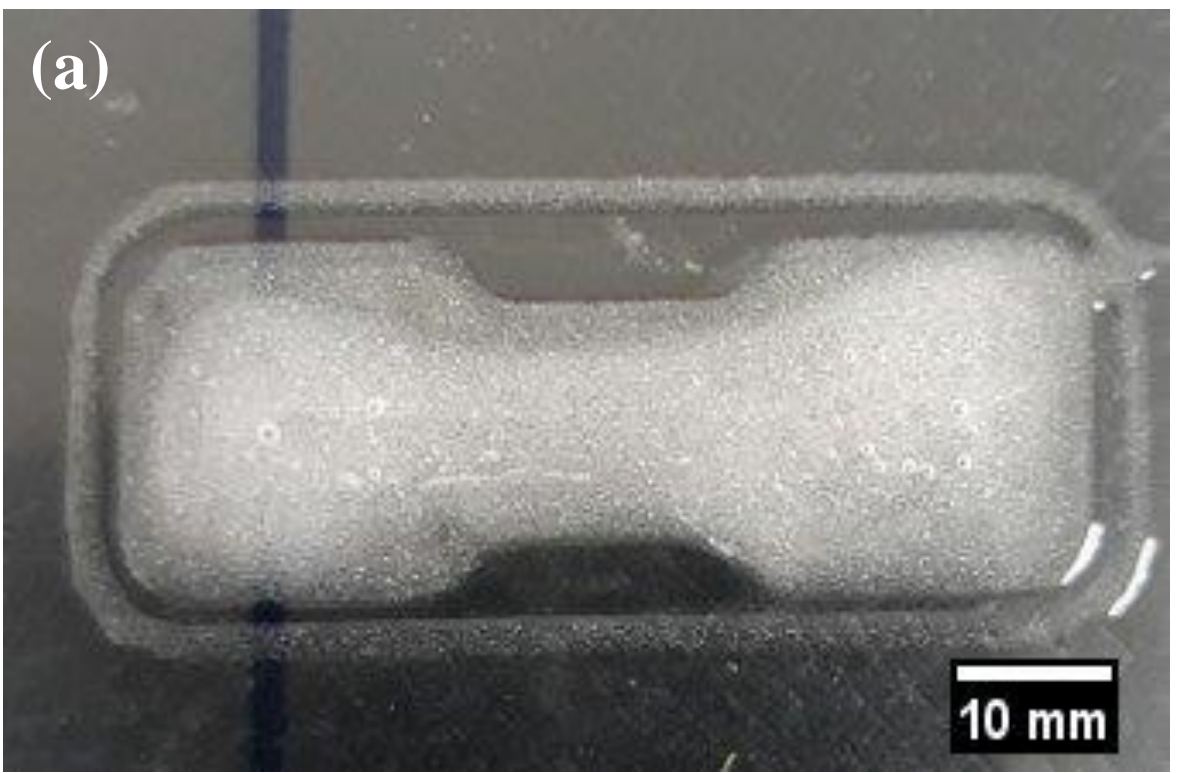




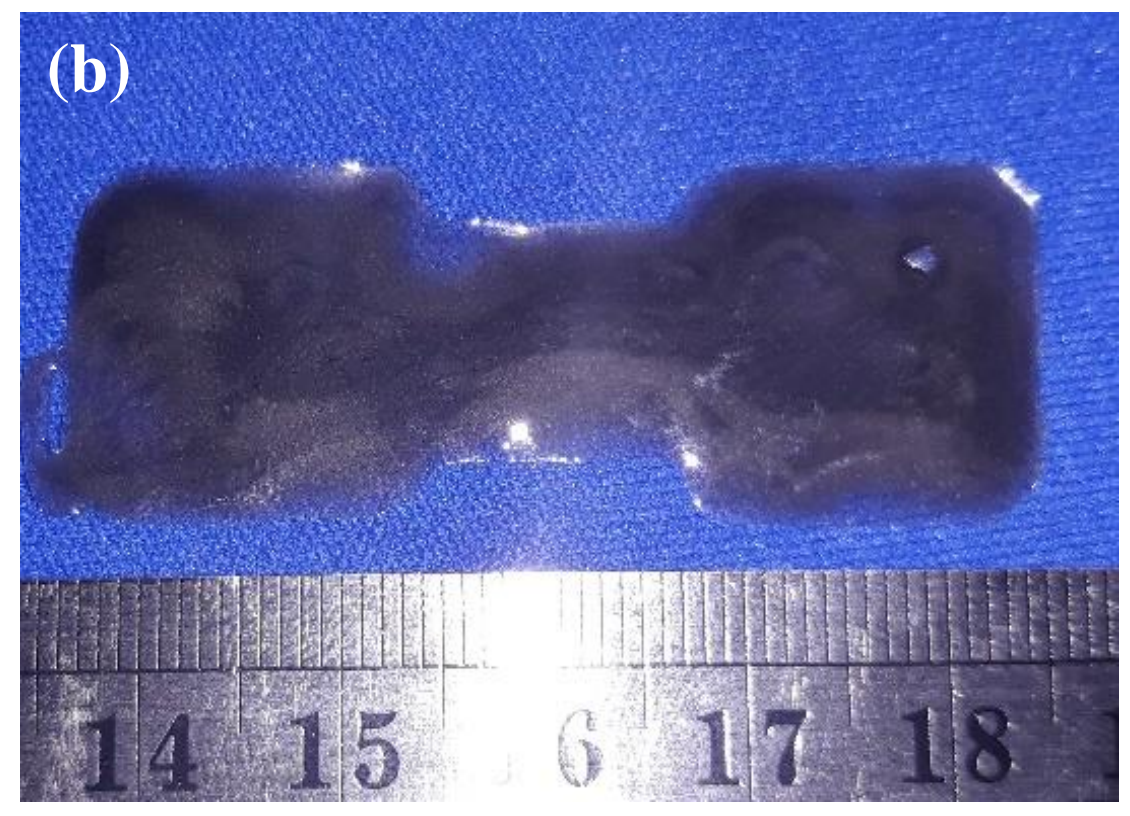

Figure 62: Images of 3D printed dog-bone shape sample placed under nearly cryogenic condition. a) SMP epoxy b) SMP epoxy-0.1 wt.\% GNP

Also, good printing of dog-bone shape of SMP epoxy-GNP is influenced by control process parameters associated with extrusion 3D printing. Such primary parameters include nozzle scanning speed during extrusion, build-plate temperature, and gap between build layer and nozzle tip. Table 2 shows the parameter values for the 3D printing of SMP epoxy dog-bone shape parts. Selection of these parameter values is very critical in obtaining a good quality print. For instance, nozzle speed can greatly affect dimensional accuracy. It is observed that high nozzle speed can lead to vibration of 3D printer, resulting in a relative error of printed components. As a result, the printing parameter values used in this study agrees with the literature study on 3D printing of low viscous conventional polymers [157]. 
Table 6.1. Printing parameters of 3D printed SMP epoxy-0.1 wt.\% GNP Composites

\begin{tabular}{|l|c|}
\hline Parameters & Values \\
\hline Nozzle scan speed while extruding $(\mathrm{mm} / \mathrm{s})$ & 22 \\
\hline Bedplate temperature $\left({ }^{\circ} \mathrm{C}\right)$ & 0.2 \\
\hline $\begin{array}{l}\text { The gap between the first layer and the nozzle tip } \\
(\mathrm{mm})\end{array}$ & 100 \\
\hline Print infill density $(\%)$ & \\
\hline
\end{tabular}

\subsection{Morphology of 3D printed SMP epoxy-graphene nanoplatelets powder (GNP)}

The morphology of 3D printed SMP epoxy and SMP epoxy-0.1 wt.\% GNP composites is examined from their top and side view surfaces (figure 63). Figures 63a and b show SEM micrographs of top surfaces of SMP epoxy. SEM micrographs of side view surface of SMP epoxy are displayed at low and high magnifications (figures $63 \mathrm{c}$ and d). SMP epoxy (control sample) reveals smooth surface with stripes that could have stemmed from the infilling pattern at $45^{\circ}$ (figure 63a). At higher magnification, SMP epoxy further revealed directionality at almost $45^{\circ}$ of the non-crystalline or amorphous arrangement of epoxy chains oriented. It could also possibly be due to influence of the infilling of the gauge length (filled at $45^{\circ}$ ) during $3 \mathrm{D}$ printing. Figures $63 \mathrm{c}$ and d show smooth and slightly rough surface regions of SMP epoxy. Higher magnification micrograph shows that there exist largely smooth surface regions in between the rough surface regions. This typical surface characteristics of thermoset epoxy consisting of disordered chains. 


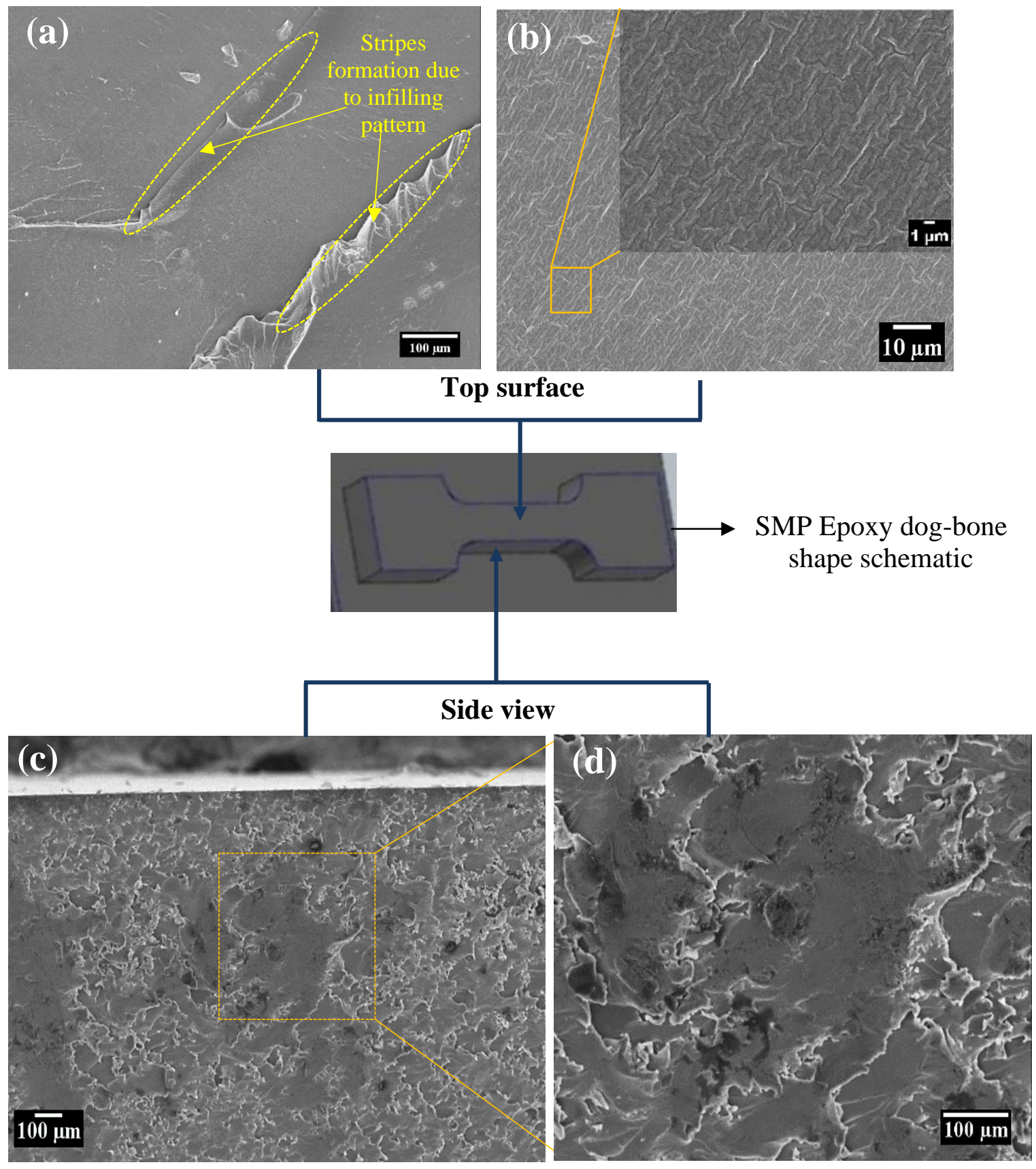

Figure 63: SEM micrographs of cross-section of 3D printed dog-bone shape specimen from SMP epoxy top view surface (a) at lower magnification $(150 \times)$; (b) at higher magnification $(1500 \times$ and $5000 \times$ [inset] $)$ and side view surface $(c)$ at lower magnification $(100 \times)$ and $(d)$ at higher magnification $(750 \times)$ 

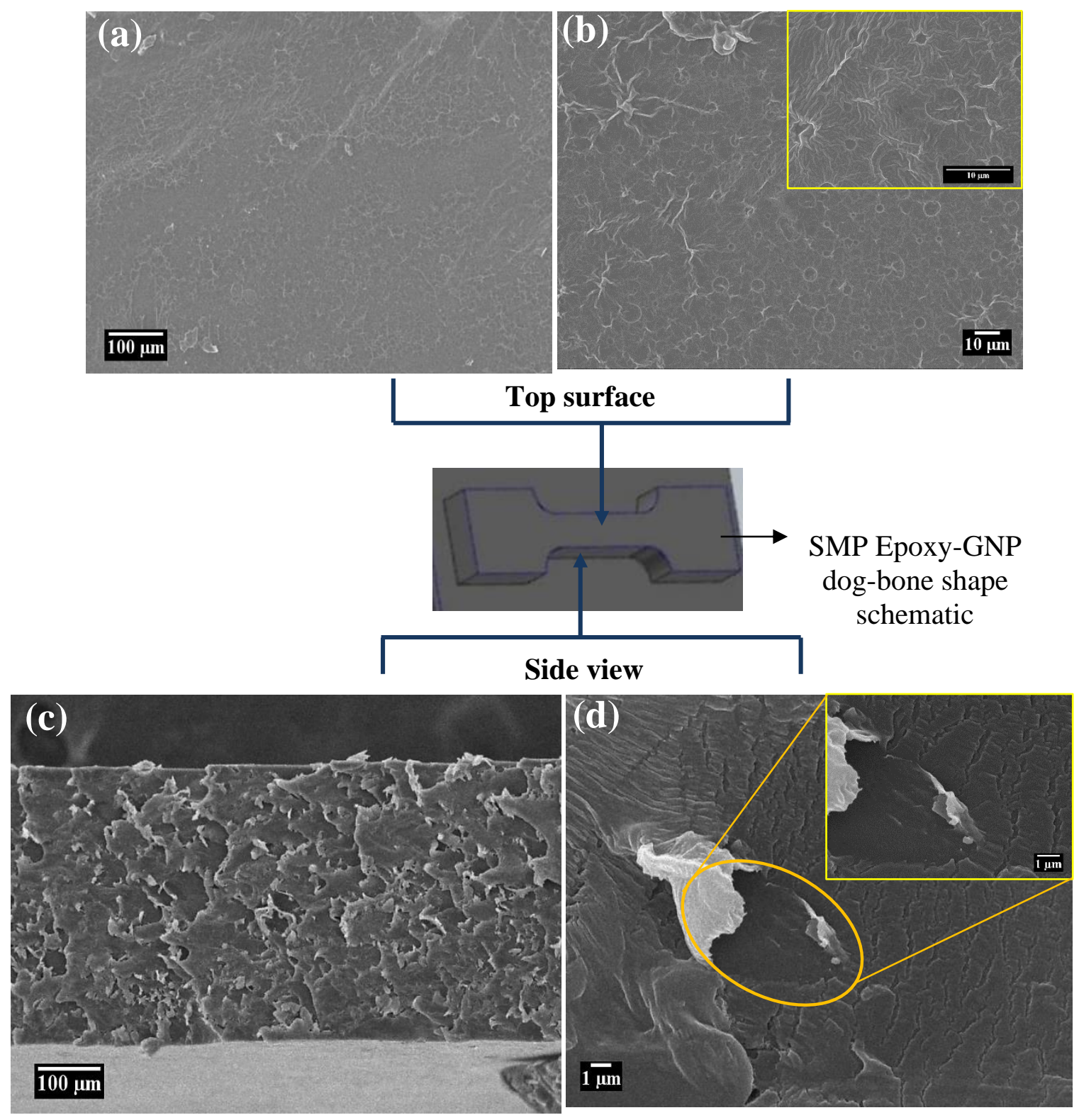

Figure 64: SEM micrographs of cross-section of 3D printed dog-bone shape specimen from SMP epoxy-0.1 wt.\% GNP (a) at lower magnification $(150 \times)$; (b) at higher magnification $(1500 \times$ and $5000 \times$ [inset] $)$ and SMP epoxy-0.1 wt.\% GNP (c) revealing rough surfaces and (d) interface between graphene and SMP epoxy

Furthermore, figures $64 \mathrm{a}-\mathrm{d}$ show the top and side view surfaces of 3D printed dogbone shape SMP epoxy-0.1 wt.\% GNP composite. Unlike that of the SMP epoxy sample, the top surface of the composite sample shows dendritic patterns (figure 64a) due to GNP 
powders added into the polymer matrix. The top surface of SMP epoxy-GNP composite shows graphene dispersed in some regions having a rough surface. Few regions show smooth surfaces. It indicates that uniform GNP dispersion is noticed in some regions of SMP epoxy matrix and non-uniform GNP distribution in a few other regions. It also shows that the amount of GNP added is suitable for the nozzle inner diameter $(800 \mu \mathrm{m})$ and print speed $(30 \mathrm{~mm} / \mathrm{s})$ to perfectly extrude the precursor. This is because higher GNP weight percent can cause nozzle clogging, largely resulting in non-uniform GNP distribution in the printed sample. Tiny air bubbles and non-directionality in the epoxy chains was also observed at higher magnification (figure 64b). The air bubbles could be the air trapped during mixing of SMP epoxy and GNP powders, and during partial curing of the composite. The inset at higher magnification $(5000 \times)$ clearly shows some regular arrays of SMP epoxy chain within its overall disoriented system after GNP addition. This indicates that GNP reinforcement partially aids in the alignment of the chain molecules of SMP epoxy.

The significant irregular protuberances or rough surfaces observed on the side view surface (figure 64c) could be attributed to the embedded GNP that is strongly bonded with SMP epoxy, with no nanolayer pull out. Figure 64d shows SMP epoxy well coated on the GNP and less graphene pull out from the matrix. This indicates excellent interface between SMP epoxy and GNP. This results in strong physical bonding that can influence the mechanical property of SMP epoxy-GNP composite. Additional SEM fractographs are shown in the section of mechanical property of the 3D printed samples (section 6.6.1). 


\subsection{Phase and Bonding Characteristics}

Raman and FTIR spectra results are obtained from the microstructural characterization to understand phase and bonding characteristics of the 3D printed SMP epoxy-based samples (figure 65a-c). Figure 65a shows Raman spectra of GNP powder obtained from previous work in our group [158]. Like GrF Raman spectra discussed in section 4.1, figure 65a unveils the three typical graphene peaks representing graphene molecular fingerprints. The D, G, and 2D peaks are observed at 1360, 1580 and $2700 \mathrm{~cm}^{-1}$, respectively. Figure $63 \mathrm{~b}$ and c present Raman spectra of SMP epoxy, SMP epoxy-0.1 wt.\% GNP and SMP epoxy0.25 wt. $\%$ GNP samples at wavenumber $1000-1800 \mathrm{~cm}^{-1}$ and $2000-4000 \mathrm{~cm}^{-1}$. SMP epoxy peaks are recognized at wavenumbers of typical epoxy peaks [159]. Some of the noticeable peaks are at 1113, 1162, 1318, 1460, 1580, and 1608 and $3069 \mathrm{~cm}^{-1}$.

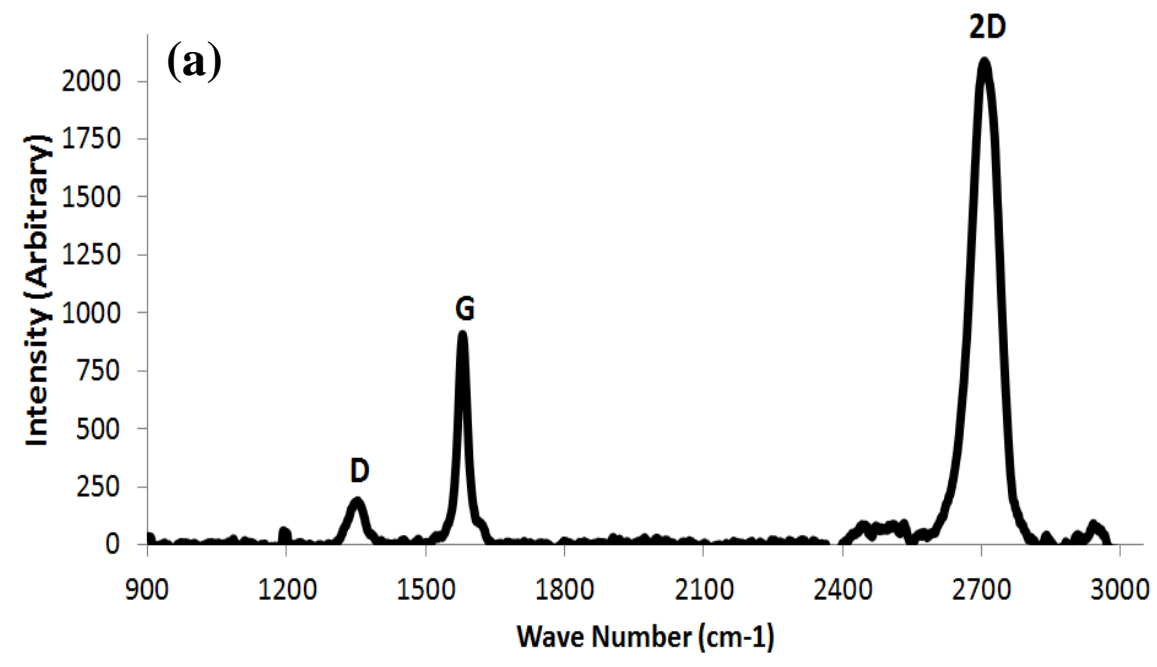



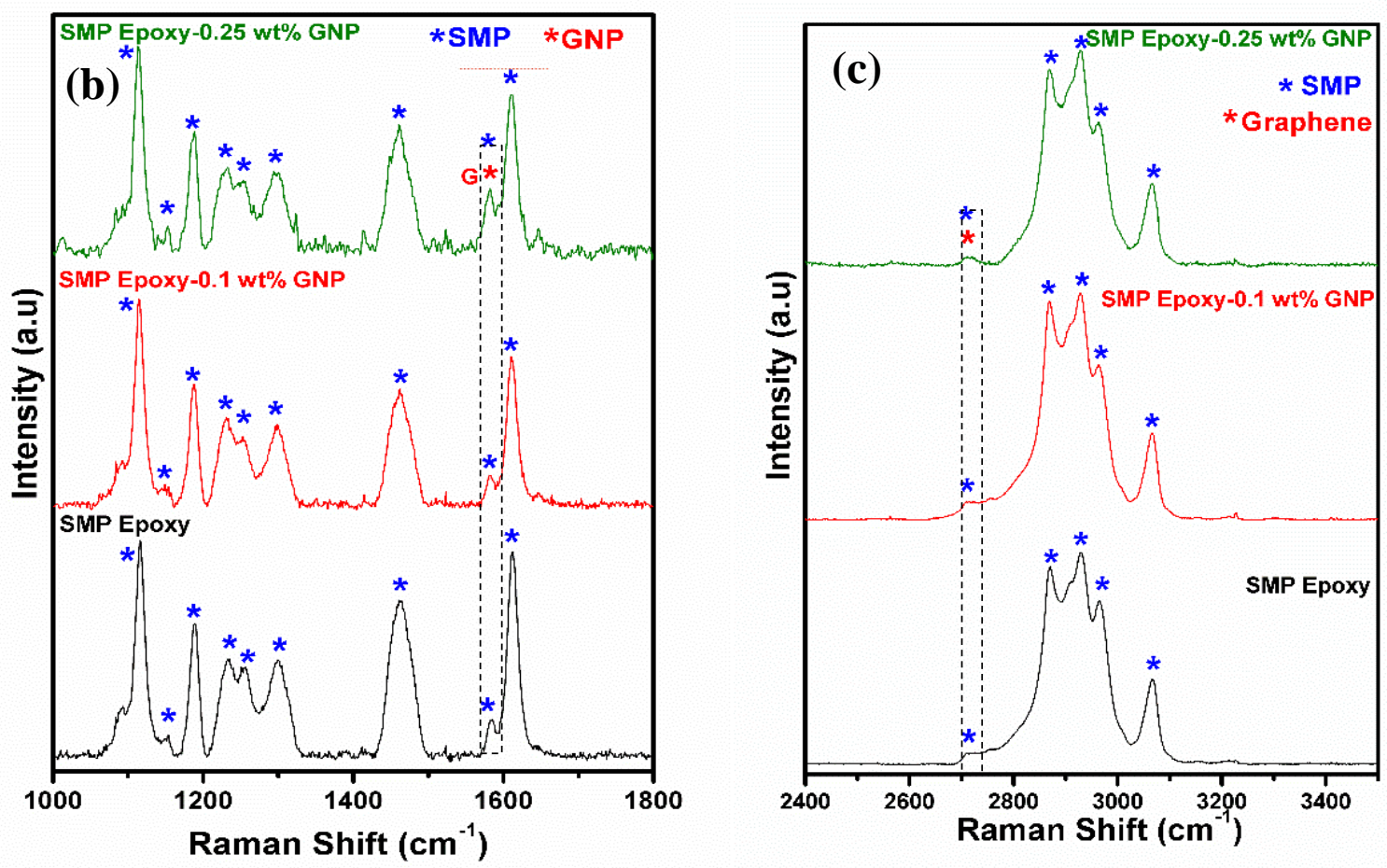

Figure 65: Raman spectrum of (a) GNP powder [158]; Raman spectra of SMP epoxy, SMP epoxy-0.1 wt.\% GNP and SMP epoxy-0.25 wt.\% GNP at (b) wavenumber $1000-1800$ $\mathrm{cm}^{-1}$; (c) at wavenumber $2000-4000 \mathrm{~cm}^{-1}$.

At these respective peaks, strong C-O-C epoxy ring, C-H waggings, $\mathrm{CH}_{3}$ bending, $\mathrm{CH}_{2}$ deformation vibration, shoulder, and strong aromatic rings stretch are observed. It is important to note that the shoulder aromatic signature from SMP epoxy appears at 1580 $\mathrm{cm}^{-1}$, which is almost the same graphitic signature from GNP.

Figure 65b reveals Raman spectra of SMP epoxy-0.1 wt.\% GNP at wavenumber 1000 $-1800 \mathrm{~cm}^{-1}$. Graphitic signature (G peak) is not detected though shoulder band was noticed at $1580 \mathrm{~cm}^{-1}$, which is similar to the Raman spectrum for SMP epoxy. G peak cannot be confirmed because the shoulder bands from the Raman spectra of SMP epoxy and SMP epoxy-0.1 wt.\% GNP has the same peak intensity. To detect the G peak, GNP content is increased from 0.1 to 0.25 wt. \%. With increased GNP content, SMP epoxy-0.25 wt.\% GNP 
Raman band reveals graphitic signature (G peak) at $1581 \mathrm{~cm}^{-1}$. The detection of the $\mathrm{G}$ peak is confirmed based on the peak intensity increase at the shoulder band $\left(1581 \mathrm{~cm}^{-1}\right)$. The ratio of the peak intensity of the shoulder peak $\left(\right.$ at $1580 \mathrm{~cm}^{-1}$ ) to strong aromatic peak (at $1608 \mathrm{~cm}^{-1}$ ) for Raman spectrum of SMP epoxy is 0.85 ; whereas, the peak intensity ratio for Raman spectrum of SMP epoxy-0.25 wt.\% GNP is 0.96 (12\% increase in peak intensity). The broad hump of D peak was not visible. It suggests that extremely low thermal treatment could facilitate the defect-free region in the graphene by coalescing of nanographene islands [86]. Good dispersion of graphene also prevents defect due to agglomeration, thereby accounting for invisible $\mathrm{D}$ peak. The wavenumber shift in $\mathrm{G}$ peak of GNP (figure 65b) could be attributed to slight shrinkage that occurs during polymerization of the SMP epoxy chains. Presence of 2D peak could be observed in 0.25 wt. $\%$ GNP composition, as shown in figure $65 \mathrm{c}$. The $2 \mathrm{D}$ peak intensity at this composition is slightly higher than at $0.1 \mathrm{wt} . \%$ GNP and SMP epoxy.

In addition to the Raman studies, FTIR spectra of the 3D printed SMP epoxy and SMP epoxy-0.1 wt\% GNP composite is shown (figure 66). The absorption bands at 3348 and $2970 \mathrm{~cm}^{-1}$ are ascribed to saturated $\mathrm{O}-\mathrm{H}$ and $\mathrm{C}-\mathrm{H}$ stretching vibrations. Bending vibration of ether $\mathrm{C}=\mathrm{O}$ corresponds to the characteristic absorbance peak at $1100 \mathrm{~cm}^{-1}$ while the peak at $1535 \mathrm{~cm}^{-1}$ can be attributed to stretching vibration of N-H. High peak intensity displayed by $\mathrm{C}-\mathrm{H}$ and $\mathrm{N}-\mathrm{H}$ is due to greater change in dipole moment concerning distance than that of $\mathrm{O}-\mathrm{H}$ and $\mathrm{C}-\mathrm{O}$ functional groups. It is observed that the peak width of SMP epoxy-0.1 wt $\%$ GNP decreased at absorption band $3348 \mathrm{~cm}^{-1}$ and slightly increased at absorption band 1535 and $2970 \mathrm{~cm}^{-1}$ compared with that of SMP epoxy. 


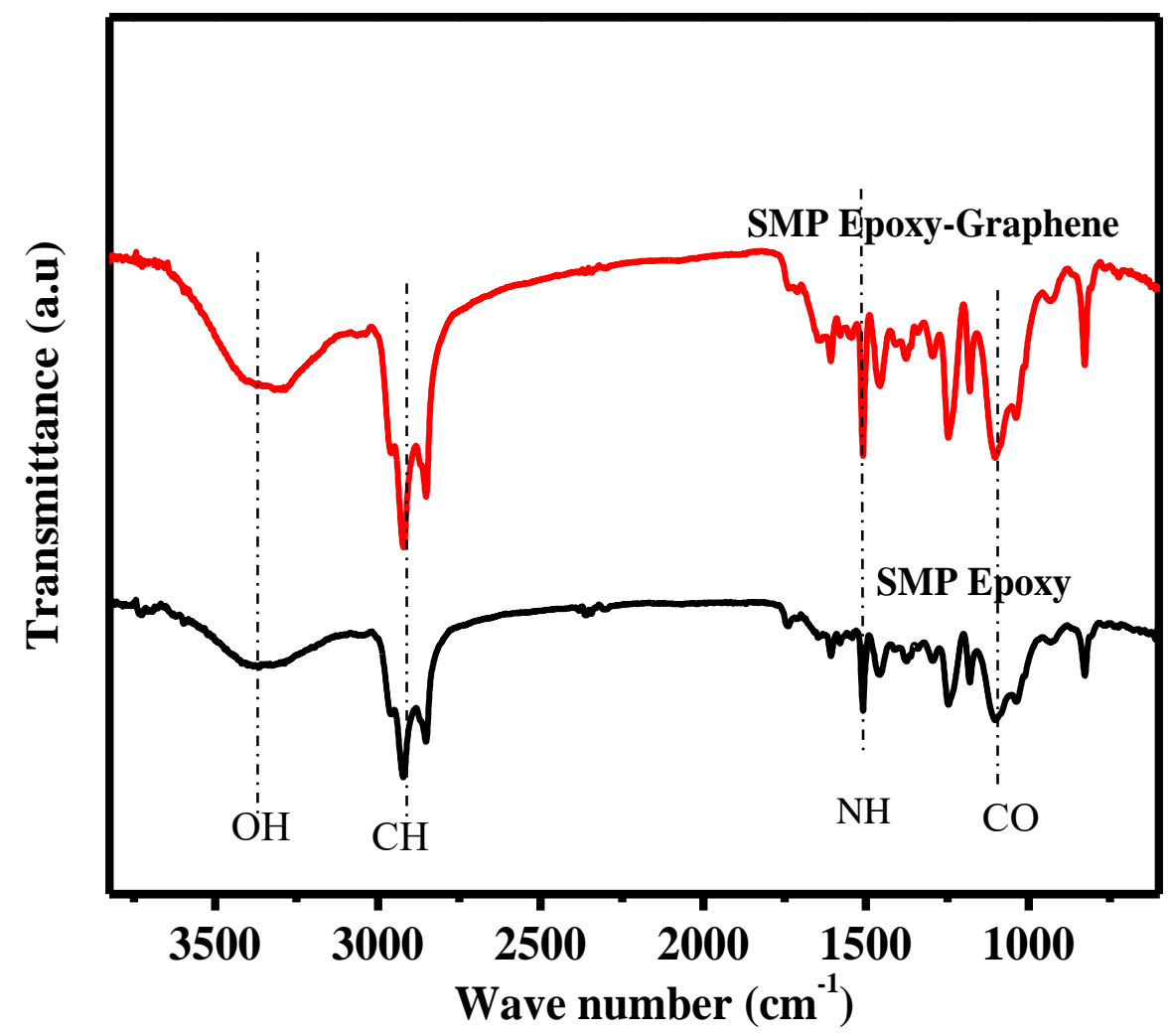

Figure 66: FTIR spectra of SMP epoxy and SMP epoxy-0.1 wt.\% GNP composite confirming the possibility of interfacial interactions between functionalities on SMP epoxy and graphene

This construes that less hydrogen bonding or interaction occurs at $3348 \mathrm{~cm}^{-1}$ and some intermolecular interactions at 1535 and $2970 \mathrm{~cm}^{-1}$. Furthermore, FTIR results of cast sample of SMP epoxy-GrF and 3D printed sample SMP epoxy-GNP are compared. Cast sample displayed higher peak intensity than its 3D printed counterpart. This could be attributed to more concentration of molecules in the cast sample, which has higher thickness $(3 \mathrm{~mm})$ than $3 \mathrm{D}$ printed sample $(0.6 \mathrm{~mm})$. Wider peak widths assigned to $\mathrm{C}-\mathrm{H}$ and $\mathrm{N}-\mathrm{H}$ of the cast samples indicates more hydrogen bond interactions of $\mathrm{C}-\mathrm{H}$ and $\mathrm{N}-\mathrm{H}$ bond of $\mathrm{GrF}$ with $\mathrm{C}-\mathrm{H}$ and $\mathrm{N}-\mathrm{H}$ bond of SMP epoxy. 


\subsection{Thermal Characteristics}

Glass transition ( $\mathrm{Tg}$ ) behavior of 3D printed SMP epoxy-based samples is investigated, using SDT Q600 instrument, after completely curing the 3D printed dog-bone shape room temperature. Figure 67 presents the $\mathrm{Tg}$ of the 3D printed samples, which is subjected to the heating rate of $10^{\circ} \mathrm{C} / \mathrm{min}$ from room temperature to $175^{\circ} \mathrm{C}$.

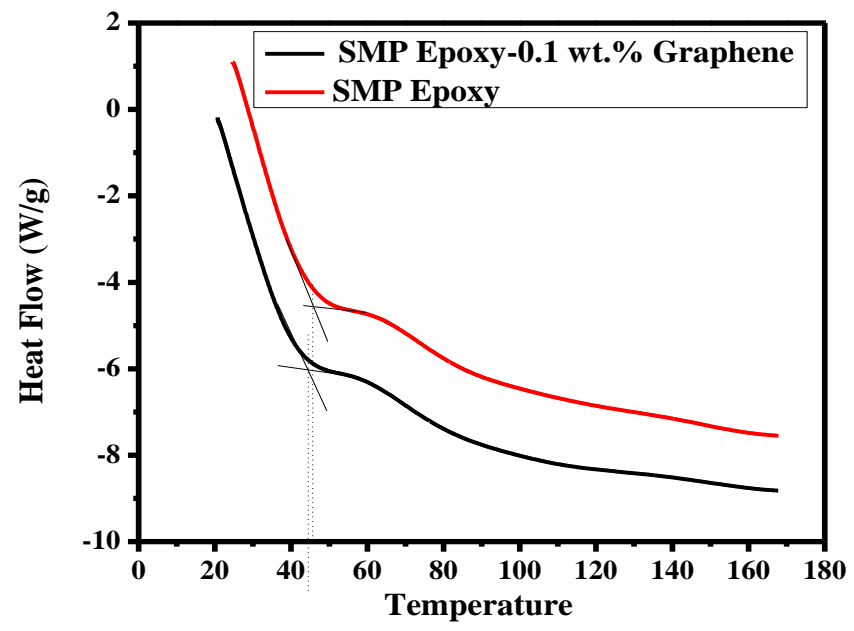

Figure 67: Differential Scanning Calorimetry (DSC) curves of 3D printed SMP epoxy and SMP epoxy-0.1 wt.\% GNP Composite

The DSC thermogram curves of SMP epoxy and SMP epoxy-GNP of both the samples showed inflection at around 45 and $44^{\circ} \mathrm{C}$, respectively. The inflection region is the $\mathrm{Tg}$ region. It also represents the small endothermic peak which can be attributed to enthalpy relaxation of the amorphous SMP epoxy from unfavorable chain orientations towards a more stable chain conformation. Figure 67 shows that SMP epoxy-GNP has a marginal Tg decrease than SMP epoxy sample. GNP addition could occlude crosslinking of the SMP epoxy chains. 
Further, low GNP content and non-uniform dispersion in some regions of the sample do not provide enough physical interaction with the SMP epoxy. Hence, these factors could result in lower Tg of the composite. Compared with $\mathrm{Tg}$ of mold cast SMP epoxy sample $\left(42^{\circ} \mathrm{C}\right.$ ) (figure 31$)$, the $3 \mathrm{D}$ printed SMP epoxy has higher $\mathrm{Tg}\left(45^{\circ} \mathrm{C}\right)$. This can be attributed to curing time (4 days) given to the 3D printed SMP epoxy (which is part of successful printing conditions). The timing enables the SMP polymer chains to find energetically favorable orientations to crosslink with neighboring polymer chains. Shear thickening behavior during 3D printing could also possibly cause enhanced intramolecular interactions that can influence the curing of the printed samples [160]. Layer height and print infill pattern are factors that could affect the Tg of the 3D printed sample. They could control the orientation of the GNP in the example. This construes that increased layer height could create an avenue for different GNP orientations. The sample layer height in this study is $0.6 \mathrm{~mm}$, which could be thin or thick depending on the applications. The height was preferred because it contributes to retaining the dog-bone shape geometry and no smudging of the SMP epoxy precursors. The layer height of the sample as an important parameter could allow for GNP orientation, but this does not significantly affect the composite Tg due to low GNP content.

\subsection{Shape Memory Behavior of 3D Printed SMP Epoxy-GNP Composite}

SMP epoxy-GrF samples fabricated by the mold-casting method have shown great capability to be thermally stimulated to exhibit shape recovery (highlighted in section 4.1). However, 3D printing of SMP component offers additional advantages such as customization, cost, and time. It is thus insightful to investigate thermal actuation and shape 
recovery performance of 3D printed SMP epoxy samples. Influence of 3D printing process parameters on the printed quality of SMP epoxy-based samples is also considered. Investigation of shape recovery of 3D printed SMP epoxy would portray the robustness of SMP epoxy resins as precursors for multiple fabrication techniques in the design of smart materials.

\subsubsection{Thermal Actuation}

Thermal stimulation of printed SMP epoxy and SMP epoxy-GNP samples with dimensions $(48 \times 1.1 \times 0.4 \mathrm{~mm})$ is performed by using hot plate heating source at a temperature of $70^{\circ} \mathrm{C}$. The stimulation propels deployment of the sample from its temporary bent shape to its permanent flat shape. Qualitative and quantitative evaluation of shape recovery of the samples is obtained from the fold-deploy tests (figure 68a and b). Figure $68 \mathrm{a}$ and $\mathrm{b}$ show the qualitative evaluation in terms of shape recovery images of bent shapes of SMP epoxy and SMP epoxy-GNP composite, respectively. The images are captured from recorded shape recovery videos of the samples during thermal stimulation.
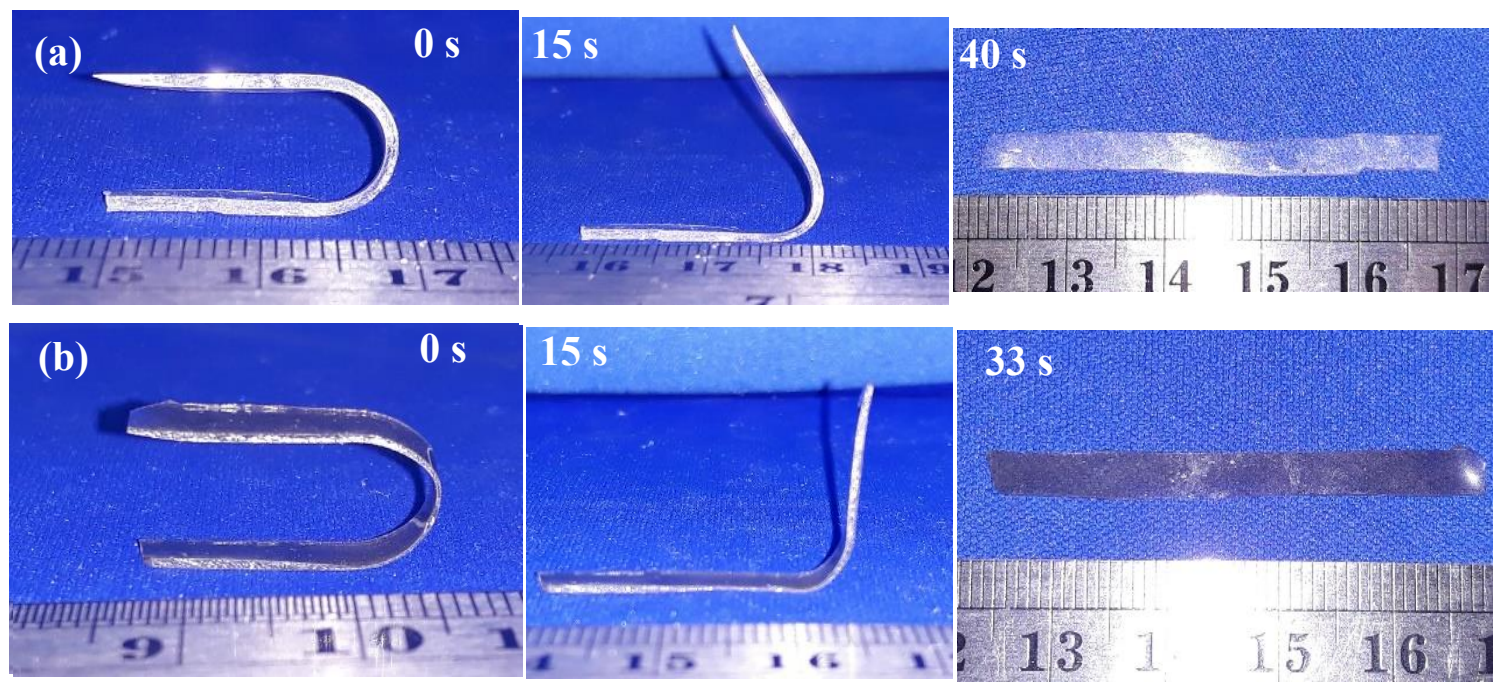

Figure 68: Shape recovery images of pre-deformed samples actuated by direct heat from hot plate (a) SMP epoxy and (b) SMP epoxy-0.1 wt.\% GNP composite 
It is noticed that both SMP epoxy and its composite recover entirely to their original shape. The recovery images thus attest that any object printed with SMP epoxy prepared in our lab through syringe-based extrusion technique can exhibit shape recovery property. Moreover, at recovery temperature of $70^{\circ} \mathrm{C}$ and $15 \mathrm{~s}$, the printed composite displays larger recovery angle compared with that of SMP epoxy. The larger recovery angle experienced by the composite can be attributed to excellent thermal conduction and high stiffness of GNP reinforcement in the SMP epoxy matrix. This implies that the composite has a faster recovery rate than the SMP epoxy. Quantification of the samples' recovery angles is further carried out (figure 69) with the aid of Image J software (version 6). Figure 69 shows the recovery angle profile concerning recovery time for the 3D printed SMP epoxy and its composite. The slope of the profile represents the recovery rate of the samples. It is observed that the slope of SMP epoxy-0.1 wt.\% GNP is higher than that of SMP epoxy. This is corroborated with the values of recovery rates between recovery time range of 10 and $25 \mathrm{~s} \mathrm{(as} \mathrm{marked} \mathrm{on} \mathrm{the} \mathrm{profile).}$ 


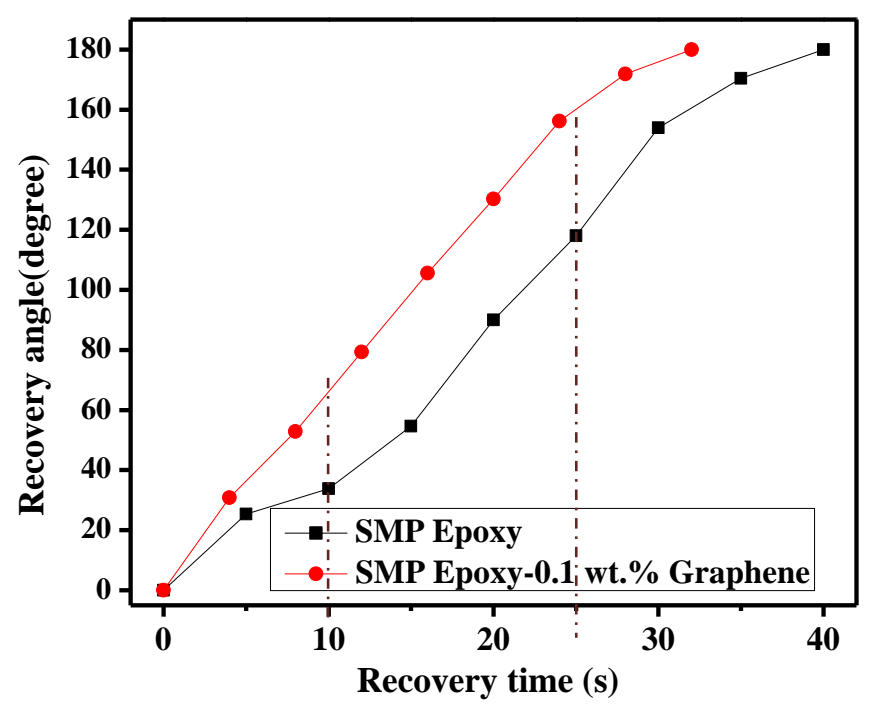

Figure 69: Shape recovery images of pre-deformed SMP epoxy and SMP epoxy-0.1 wt.\% GNP composite samples actuated by direct heat from the hot plate heat source

Recovery rates of SMP epoxy and SMP epoxy-GNP composites are 4.3 and 5.0 degree/s, respectively. This indicates that recovery rate performance of the composite is $\sim 16 \%$ higher than that of SMP epoxy. Also, shape recovery ratios of the printed samples are obtained from the shape recovery equation (highlighted in section 4.1). Like that of the mold-cast samples, the printed SMP epoxy-GNP sample exhibited 100\% shape recovery. It thus portrays that $3 \mathrm{D}$ printed samples can also demonstrate full shape recovery.

Like GrF reinforcement, mere 0.1 wt.\% GNP addition in SMP epoxy enhances the shape recovery rate of the 3D printed composite. It shows the efficacy of graphene-based reinforcement in 3D printed SMP generally. GNP efficacy can be ascribed to its intrinsic property, such as its high thermal conductivity and stiffness. Also, less agglomeration of GNP helps in improving heat transfer within the matrix system, contributing to recovery rate improvement of SMP epoxy-GNP system. It is important to highlight that GNP 
addition synergizes well with the SMP epoxy precursor viscosity and process parameters values to produce printed parts of good quality. Achieving good quality $3 \mathrm{D}$ print by extrusion technique provides near-net-shape SMP parts. This is a boost over a mold-cast approach, which sometimes does not produce a near-net-shape components. Overall, like mold-casting, 3D printing of SMP epoxy-based samples by syringe extrusion can exhibit shape recovery behavior. This makes 3D printing technique to offer additional option over mold-casting approach when it comes to manufacturing of thin SMP parts such as thin sheets for kirigami or origami design for aerospace and electronic purposes.

\subsection{Static and Dynamic Mechanical Properties}

\subsubsection{Uniaxial Tensile Behavior}

Static mechanical behavior of 3D printed SMP epoxy-based samples is investigated. 3D printed dog-bone shape samples of SMP epoxy and GNP reinforced $(19 \times 0.4 \times 0.6 \mathrm{~mm})$ are used for the tensile tests. Images of the 3D printed SMP epoxy and GNP-based samples are shown in figure 70a and $\mathrm{b}$. Tensile properties of the dog-bone shape samples are obtained from the stress-strain plot, which are deduced from loaddisplacement curves. 


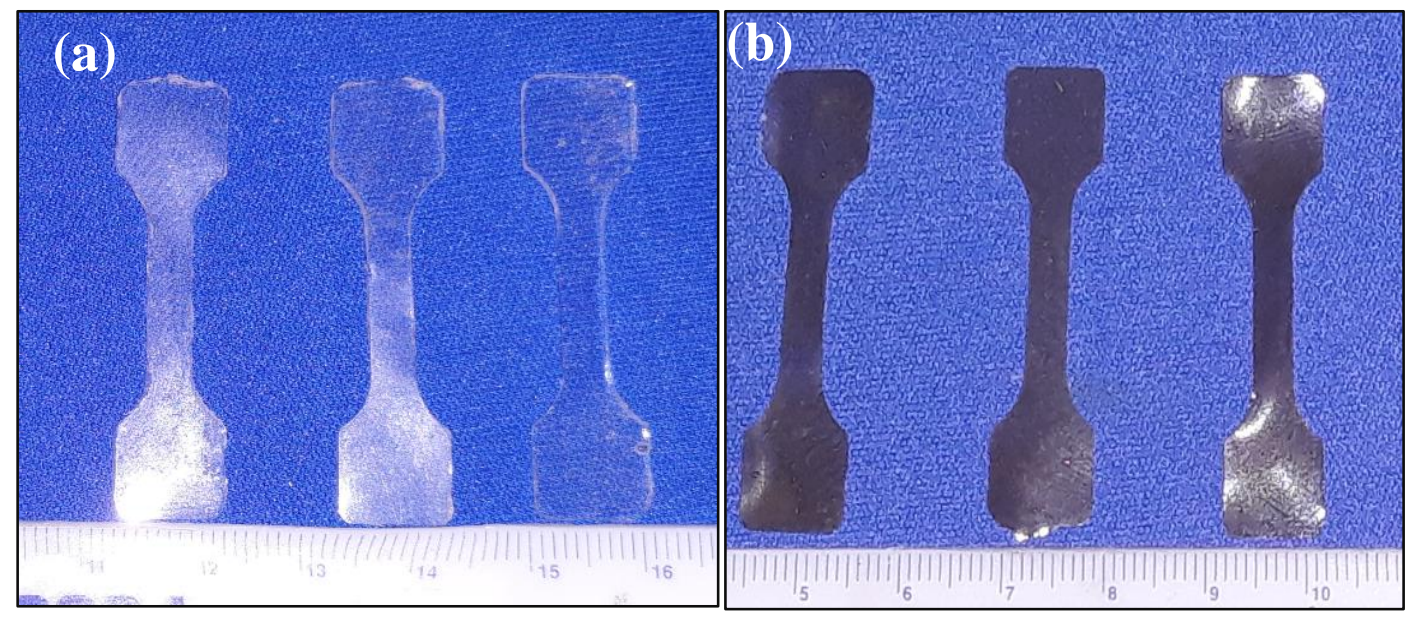

Figure 70: Images of 3D printed dog-bone shape samples fabricated by using syringe extruder and room-temperature cured (a) SMP epoxy and (b) epoxy-GNP.

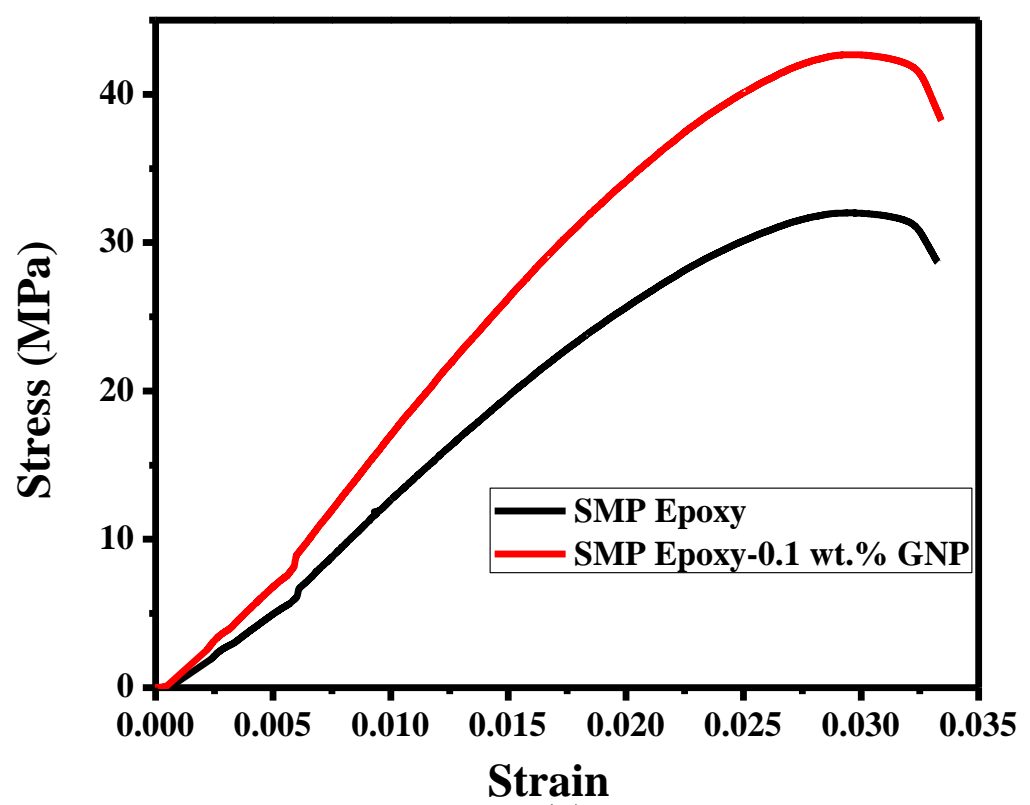

(a) 


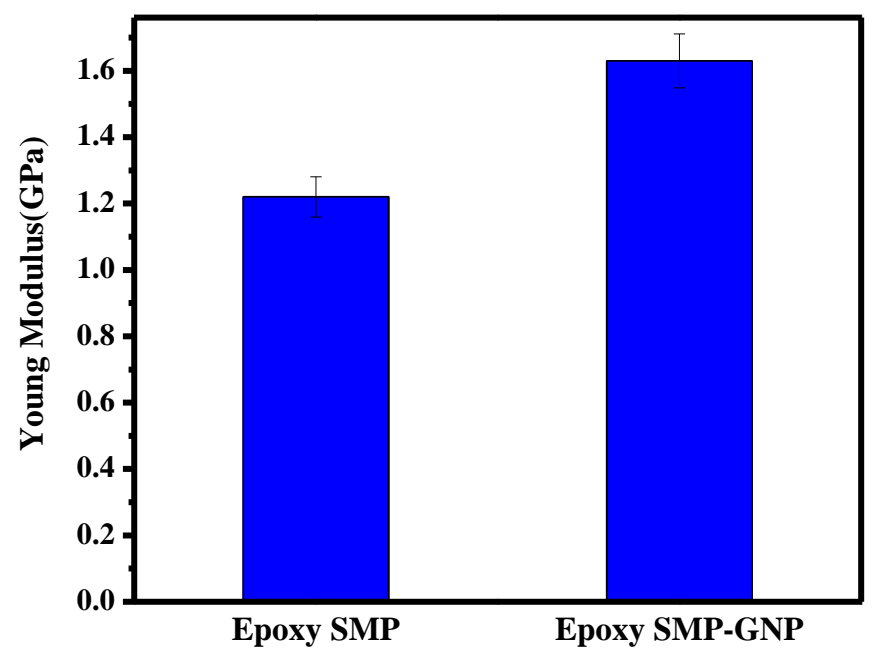

Figure 71: (a) Uniaxial tensile stress-strain curves for mold-cast SMP epoxy and SMP epoxy composite of 0.1 wt.\% GNP content (b) Comparison of Young modulus of SMP epoxy and composite of $0.1 \mathrm{wt} . \%$ GNP content

Figure 71a and b show the stress-strain profile and Young modulus comparison of the printed SMP epoxy and SMP epoxy-0.1 wt.\% GNP specimens, respectively. The corresponding mechanical properties deduced from the tensile test are displayed in Table 6.3 .

Table 6.3. Tensile Properties of SMP Epoxy and SMP Epoxy-0.1 wt.\% GNP Composite

\begin{tabular}{|l|c|c|c|}
\hline Sample & $\begin{array}{c}\text { Elastic Modulus } \\
(\mathbf{G P a})\end{array}$ & $\begin{array}{c}\text { Ultimate Tensile } \\
\text { Strength }(\mathbf{M P a})\end{array}$ & $\begin{array}{c}\text { Strain at } \\
\text { failure (\%) }\end{array}$ \\
\hline SMP Epoxy & $1.22 \pm 0.061$ & $31.20 \pm 1.56$ & 3.22 \\
\hline SMP Epoxy-GNP & $1.63 \pm 0.082$ & $41.85 \pm 2.09$ & 3.18 \\
\hline
\end{tabular}

SMP epoxy sample receives approximately $17 \%$ boost in its elastic modulus after GNP addition. SMP epoxy-GNP composite also demonstrates approximately $30 \%$ enhancement 
in the ultimate tensile strength compared to SMP epoxy. Strain to failure of SMP epoxy$0.1 \mathrm{wt} . \%$ GNP relatively remain unchanged, having a marginal increase than the control sample. In addition to results of the stress-strain response, SEM evaluation of fracture surfaces of printed SMP epoxy-GNP tensile sample is conducted. The fractographs are useful to examine the influence of the printing process parameters and strengthening mechanisms on the surface feature of the printed samples (figure 72). Figure 72 shows the SEM fractograph of 3D printed SMP epoxy taken on the planes parallel to the layer deposition axis. A smooth surface is revealed, indicating that splitting occurs along a definite plane. Similarly, smooth surface features from the cleavage of the plane are observed when taken on the planes perpendicular to the layering axis. It implies that in both parallel and perpendicular direction, the print infill angle $\left(45^{\circ} \mathrm{C}\right)$ to the layering axis does not influence or alter the brittle surface characteristics of SMP epoxy.

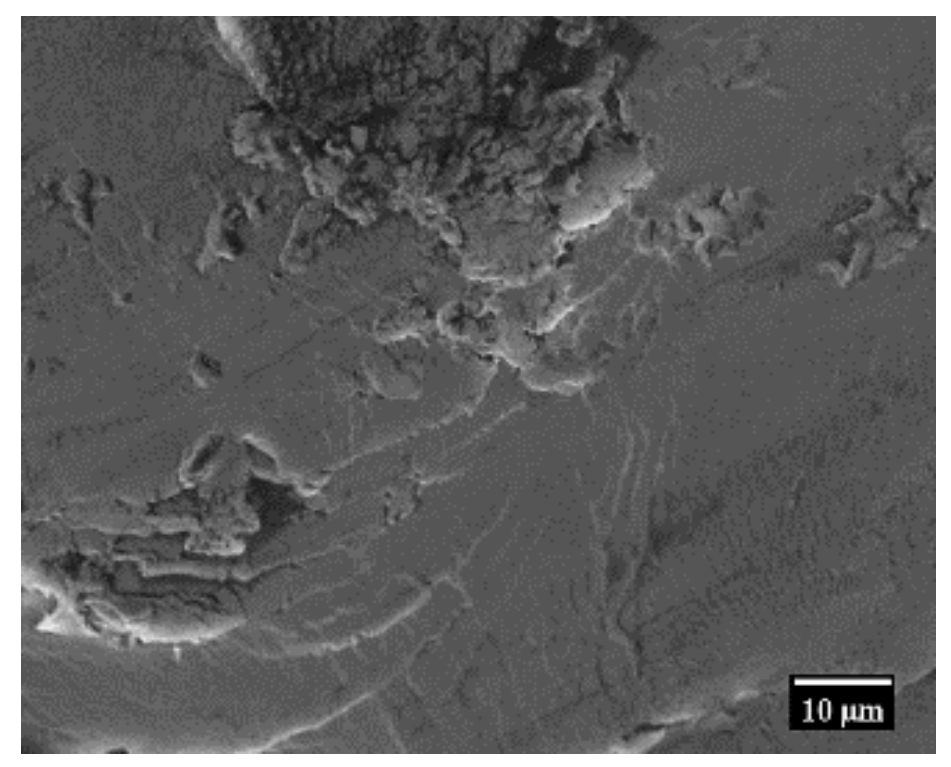

Figure 72: SEM micrographs of a cross-section of 3D printed SMP epoxy revealing smooth surface feature 
Figures 73a and $\mathrm{b}$ present SEM fractographs of 3D printed SMP epoxy-GNP taken on the planes perpendicular and parallel to the layer deposition axis, respectively. Good interface bonding of GNP with the matrix is noticed from irregular protuberances of SMP epoxyGNP fracture surface (figure 73a). The good interface facilitates excellent stress transfer from the matrix to the GNP. This prevents early matrix failure (in terms of crack initiation and propagation) while withstanding the applied tensile load. During 3D printing, appropriate distance $(0.2 \mathrm{~mm})$ between build layer and nozzle tip of the syringe extruder could also contribute to the good interface achieved. Appropriate distance would prevent the nozzle from scratching the build layer as this can create additional microvoids between GNP and SMP epoxy, resulting in weak interface. Figure 73b, obtained from the perpendicular plane, shows a feather marking feature of GNP coated with SMP epoxy. The feature also contains step-like line patterns. Print infill angle $\left(45^{\circ} \mathrm{C}\right)$ could influence GNP orientation, giving it bowing line patterns (figure 73b). The bowing lines suggest the occurrence of crack pinning of the matrix, accounting for UTS increase experienced by the composite.
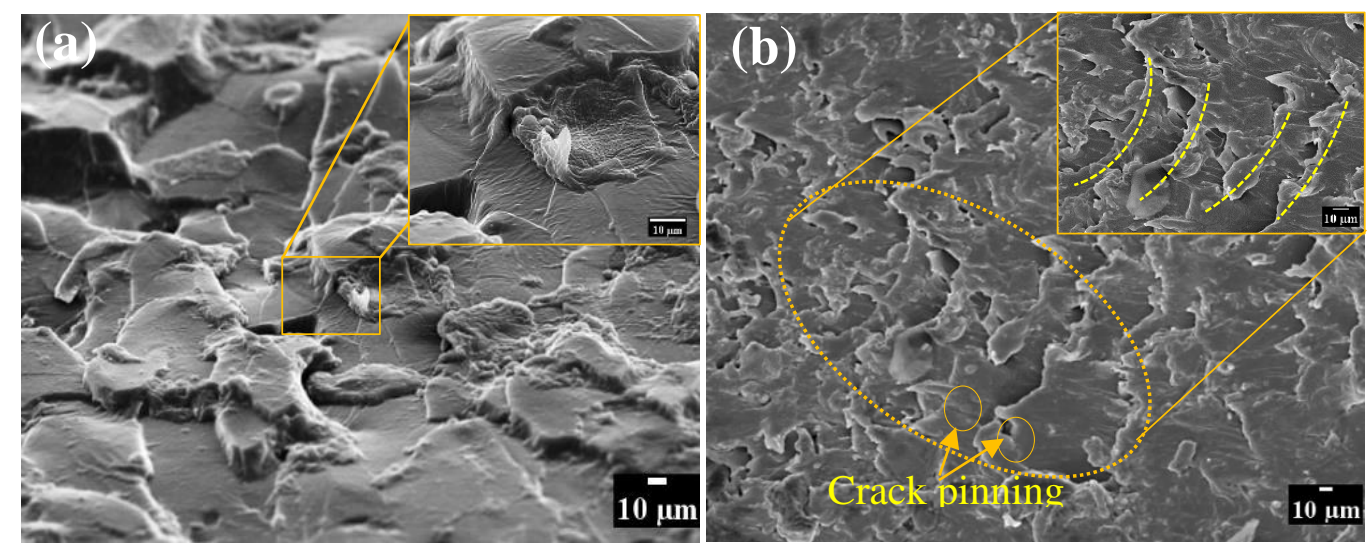

Figure 73: SEM fractographs of SMP epoxy-GNP composite. (a) image taken from perpendicular planes to the layer deposition axis, with 2200× [inset] (b) image taken from parallel plane to the layer deposition axis, with $750 \times$ [inset] 
Furthermore, figures 74a and $\mathrm{b}$ reveal GNP positioning in SMP epoxy. GNP serves as a crack bridging agent to SMP epoxy marked with propagated crack. High in-plane stiffness of GNP holds the structural integrity together of SMP epoxy and prevents the complete fracture of SMP epoxy. This portrays the great potential of GNP in SMP composite fabricated by $3 \mathrm{D}$ printing through syringe extruder.
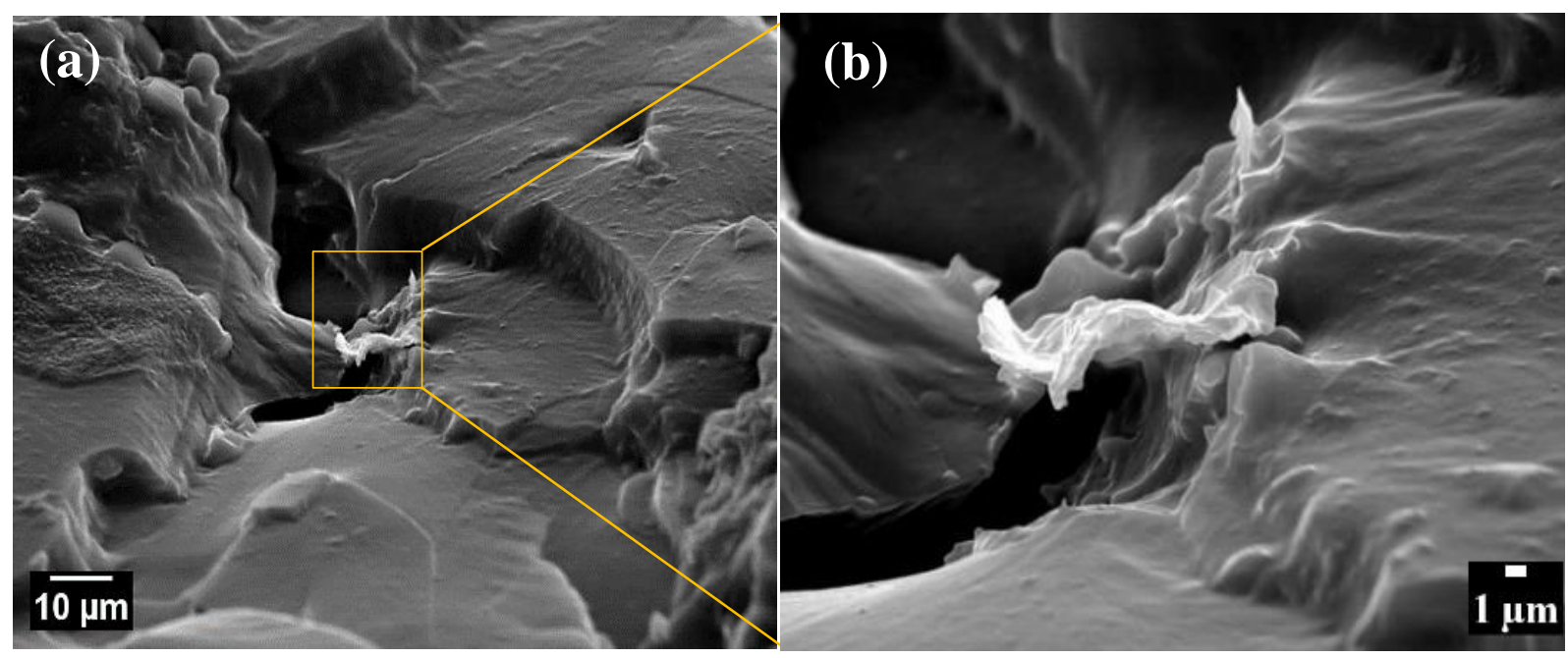

Figure 74: SEM images of the fracture surface showing the crack-bridging mechanism in SMP epoxy-GNP composite. (a) low magnification of $1500 \times$ and (b) high magnification of $5000 \times$

\subsubsection{Dynamic Mechanical Properties}

DMA characterization is performed on 3D printed SMP epoxy-based samples with dimensions $(10.70 \times 6.78 \times 0.48 \mathrm{~mm})$. The samples are subjected to tensile deformation mode because of its small thickness, which is unsuitable for 3-point bending deformation. A small amplitude load of $5 \mu \mathrm{m}$ is applied within temperature range from $24-80^{\circ} \mathrm{C}$ at heating rate of $3 \mathrm{~K} / \mathrm{min}$ and $1 \mathrm{~Hz}$. Figure $75 \mathrm{a}$ and $\mathrm{b}$ shows the dynamic storage moduli and loss tangent of SMP epoxy ( 300 MPa; 0.9) and SMP epoxy-0.1 wt.\% GNP composite printed $(\sim 1400 \mathrm{MPa} ; 1.5)$ at infill angle $\left(45^{\circ}\right)$, in rectilinear pattern and infill density 
(100\%), respectively. The plot of storage moduli and loss tangent of the printed SMP epoxy-based samples are like dynamic mechanical properties profile of the mold-cast samples. Storage moduli of the samples display typical steep decrease from 24 to $80^{\circ} \mathrm{C}$ (figure 75a). Printed SMP epoxy-0.1 wt.\% GNP composite exhibits higher storage modulus (5 times greater) than that of the SMP epoxy. Loss tangent of the samples shows a bell curve profile; it increases to the peak and decreases to zero value (figure 75b). Higher loss tangent is also displayed by SMP epoxy-0.1 wt.\% GNP compared with SMP epoxy.

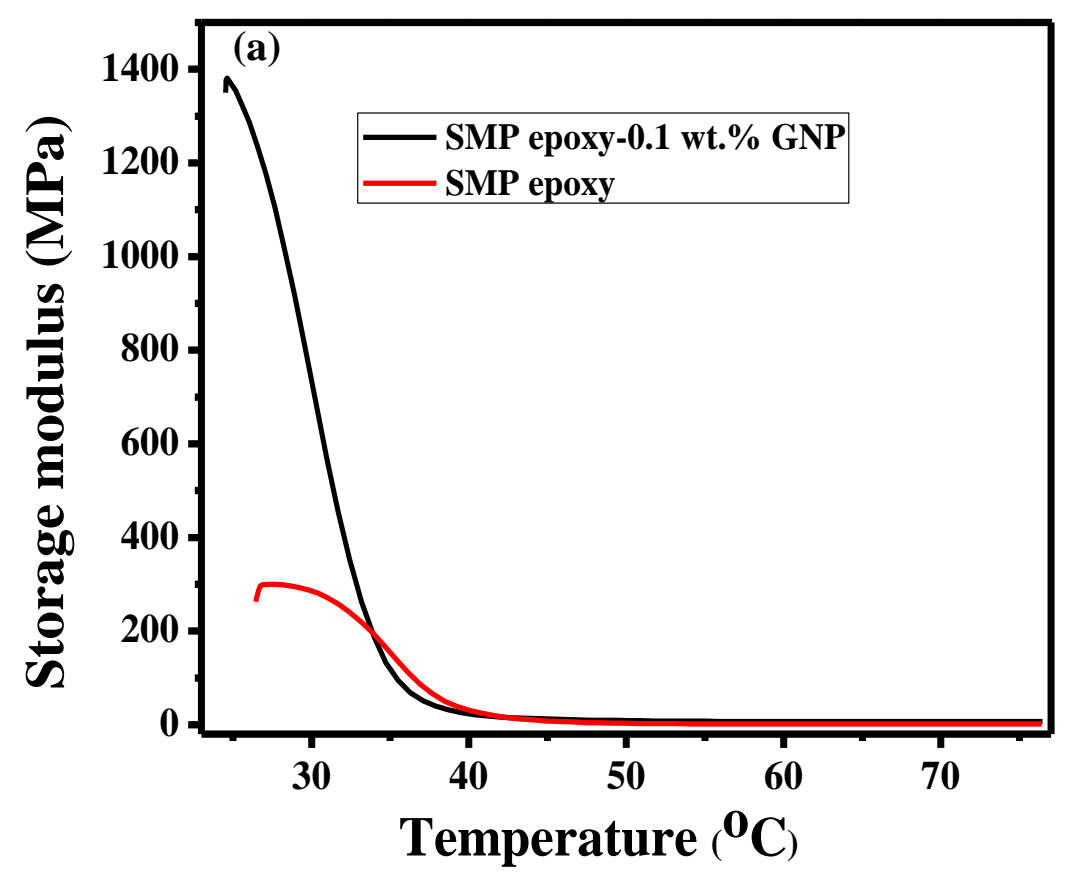




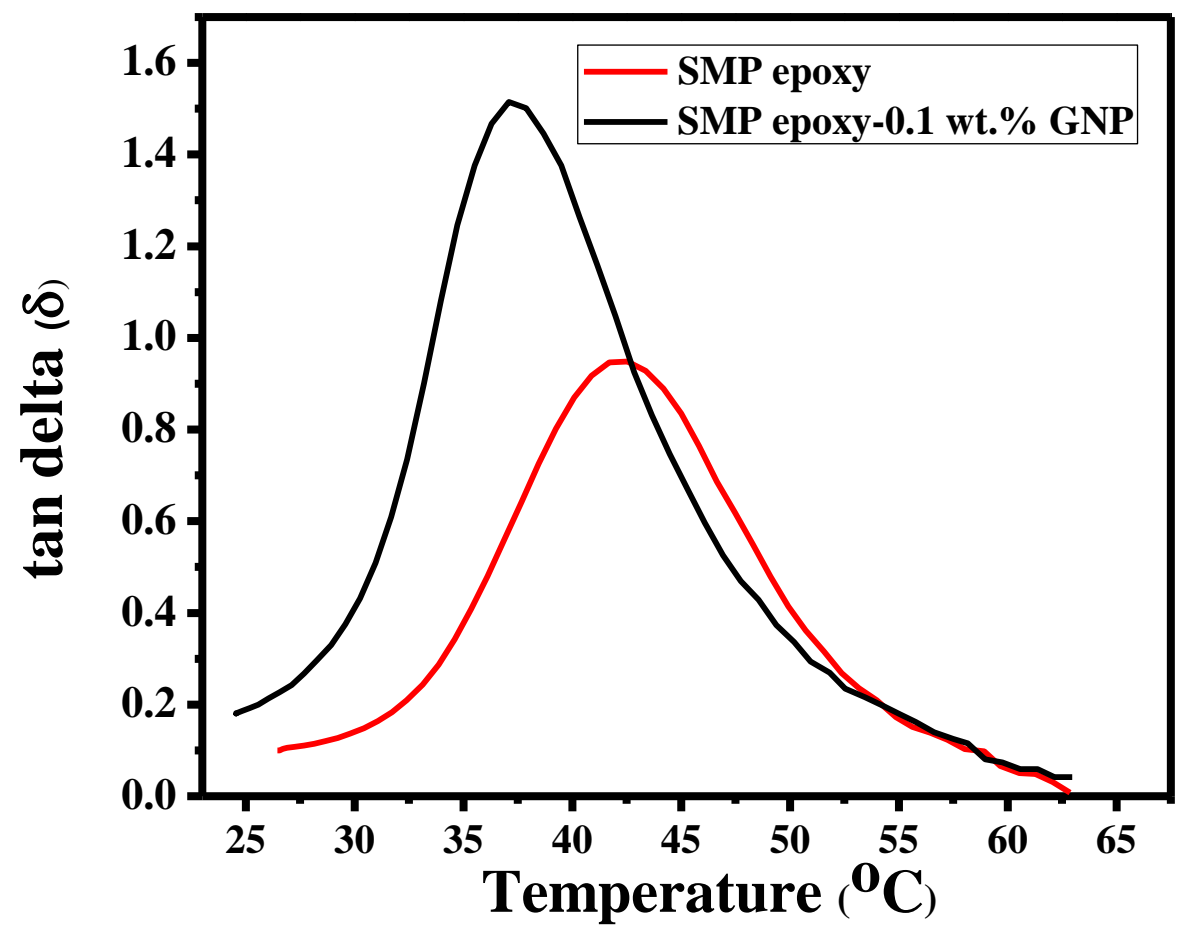

Figure 75: Plot of (a) storage modulus and (b) tan delta of SMP epoxy and SMP epoxy0.1 wt.\% GNP composite at amplitude load of $5 \mu \mathrm{m}$

The steep decrease in the storage moduli of the printed samples, as temperature increases, is due to an increase in mobility of the polymer chain molecules [154]. Incorporation of GNP in SMP epoxy matrix accounts for higher storage modulus of the composite. GNP, which has functionalized edges (carbonyl and hydroxyl groups, as shown in figure 66), creates excellent adhesion at the epoxy-GNP interface. The interface allows GNP to transfer its in-plane stiffness to the corresponding matrix [161]; hence an approximately five times increase in storage modulus of the composite between $24-34^{\circ} \mathrm{C}$. Also, printing a dog-bone shape part ( $<1 \mathrm{~mm}$ thickness) makes it possible for the composite to receive appreciable GNP response during tensile loading. Like the behavior of mold cast 
samples, at higher temperature, more cooperative segmental mobility is activated and dominant more than the GNP contribution. This leads to zero storage modulus value above $40^{\circ} \mathrm{C}$.

Furthermore, high loss tangent demonstrated by the composite can be attributed to the regions that showed fair GNP dispersion and (functionalized graphene) in SMP epoxy to achieve fair interface. This bonding enables elastic energy in the matrix to be transferred to graphene membrane where dissipation mechanisms (such as ripple formation and propagation, intermolecular interaction between layers and interlayer slippage) are mobilized [73]. Tan delta peak shift is observed from the SMP epoxy curve at $43^{\circ} \mathrm{C}$ to SMP epoxy- $0.1 \mathrm{wt} . \% \mathrm{GNP}$ at $36^{\circ} \mathrm{C}$ in figure $75 \mathrm{~b}$. The shift is noticeable compared with that of mold-cast samples, and it can be as a result of the curing process of the precursors. The mold-cast samples are cured with relatively high temperature $\left(100^{\circ} \mathrm{C}\right)$; whereas, the $3 \mathrm{D}$ printing precursor is cured at room temperature $\left(100^{\circ} \mathrm{C}\right)$. Typically, room curing of polymers has weakened crosslinking (since it is not heat-induced) compared with that of oven curing. Thus, curing of 3D printing SMP epoxy precursors at room temperature results is not as strong as that of oven-cured samples. This accounts for reason tan delta peak temperatures $\left(36\right.$ and $45^{\circ} \mathrm{C}$ ) for 3D printed SMP samples are lower than that of moldcast SMP epoxy samples. This is further reflected and attested in the Tg values of 3D printed SMP epoxy composite sample at $45^{\circ} \mathrm{C}$ (figure 67), which is lower than that of moldcast SMP epoxy composite at $50^{\circ} \mathrm{C}$ (figure 31 ). Also, GNP integration in some regions of the SMP epoxy matrix can also prevent crosslinking of the SMP resin monomers. This further reduces the crosslinking density; hence, the left shift of tan delta peak. 


\section{Chapter VII: Conclusions}

This dissertation presents a detailed analysis of thermal, mechanical, and shape memory properties of GrF-reinforced shape memory epoxy (SMP) composites. Investigations on mold-cast and 3D printed graphene-SMP epoxy composites show considerable improvement in their thermal conductivity and diffusivity. Static and dynamic mechanical behaviors of the composites reveal an increase in ultimate tensile strength, elastic moduli, loss tangent, and storage moduli with GrF and GNP reinforcements. Investigation of shape memory performance of the composites unveils enhancement in its shape recovery response after GrF addition. Key findings in this work positions $\mathrm{GrF}$ as a suitable reinforcement in SMP epoxy for structural and multifunctional applications, such as morphable aircraft wings, deployable solar array, stretchable piezoelectric sensor. SMP epoxy-GrF composites is a promising material for the highlighted application primarily due to its improved stiffness and enhanced recovery rate. Major conclusions on the processing-property-performance of SMP epoxy-GrF are summarized below:

- SMP epoxy easily percolates through the macroporous, hierarchal structure of GrF to form a robust and smart composite material. Since SMP epoxy has a higher infiltration factor than conventional polymers, facile mold-cast fabrication of SMPGrF composites can be easily achieved.

- Incorporation of $0.13 \mathrm{wt} . \% \mathrm{GrF}$ in SMP epoxy matrix introduces new architecture to the existing molecular framework, which is capable of tailoring glass transition temperature of the polymer. GrF integration increases the glass transition of SMP epoxy from 42 to $50^{\circ} \mathrm{C}$, resulting in $19 \%$ enhancement of glass transition of SMP 
epoxy matrix. Glass transition increase is due to enhanced physical interactions of the polymer chain molecules with $\mathrm{GrF}$ anatomy.

- Poor heat conduction of mold-cast SMP epoxy is greatly transformed to highly thermal conductive smart composite after GrF addition. SMP epoxy- 0.5 wt.\% GrF system exhibits $67 \%$ improvement in thermal conductivity and $63 \%$ increase in thermal diffusivity at recovery temperature $\left(70^{\circ} \mathrm{C}\right)$. The enhancement in the thermal properties is due to seamless phonon transfer from GrF interconnected framework to the matrix.

- Shape recovery characteristics of mold cast SMP epoxy-0.75 wt.\% GrF and 3D printed SMP epoxy-0.1 wt.\% GNP outperformed its SMP epoxy counterpart. SMP epoxy and GrF reinforced SMP epoxy composite displays complete shape recovery from its preform to the original shape. Furthermore, SMP epoxy and SMP epoxyGrF composite can be programmed into simple and complex geometry shapes due to its high recoverable strain characteristics.

- The shape recovery rate of the mold-cast and 3D printed graphene-based epoxy composites is $23 \%$ and $16 \%$ faster than the neat SMP epoxy. High intrinsic thermal and mechanical properties of $\mathrm{GrF}$ reinforcement primarily accounts for the improvement in the shape memory performance.

- SMP epoxy-GrF composite by mold-casting demonstrates $23 \%$ faster self-healable behavior which is due to its robust, versatile structure and its response to dynamic factors such as heat. 
- Mold-cast SMP-GrF sample displays 6\% and 20\% enhancement in ultimate tensile strength and elastic modulus, with mere 0.5 wt. $\%$ GrF addition. GrF branch capacity to withstand load is a proof of effective stress transfer in the composite.

- SMP epoxy has enough time space between its gel point and completes crosslinking that allows appropriate viscosity required for quality 3D printing of parts, using SMP epoxy precursor.

- 3D printed SMP epoxy-0.1 wt.\% GNP exhibits $30 \%$ and $17 \%$ appreciable improvement in tensile strength and elastic modulus, respectively. A strong interface and high in-plane stiffness of graphene are accountable for these improvements.

The specific conclusions listed above have established that processing of graphene-based SMP composite is highly feasible using mold casting and 3D printing techniques. The study also demonstrates that with the addition of graphene-based reinforcement, SMP epoxy can receive a considerable boost in its thermal, mechanical, and shape memory properties. This can make the SMP epoxy composites to serve as multifunctional smart materials that can be employed in several applications. SMP epoxy-GrF properties can be further improved by making modifications in the processing. Thus, recommendations for future work have been made to expand the boundaries of GrF reinforced SMP polymer composites (such as improvement in the fabrication quality (excellent resolution 3D print) and enhancing material property (mechanical characteristics) limit of SMP epoxy-GrF composite for aerospace wing morphing application and smart composite coatings). 


\section{Chapter VIII: Recommendations for the Future Work}

Based on the discussion and conclusions presented in the previous chapters, the following recommendations are made to further improve the properties of SMP epoxy-graphene composites and answer the new challenges.

\subsection{Optimizing GrF in mold-cast SMP Epoxy Composites}

The present work is performed with largely two compositions of GrF filler ( 0.5 and 0.75 wt.\%) in SMP epoxy. Optimizing GrF content would have a considerable effect on the mechanical and thermal performance of the composite. Property characterization of multiple graphene contents of the composites would enable researchers to determine the percolation limit of GrF in SMP epoxy cast composites. In order words, it would allow the scientific community to know the maximum amount of GrF addition after which there is no change in SMP epoxy property. This is needed to fully harness the thermomechanical properties of GrF reinforcement in the SMP matrix before degradation of the composite properties.

\subsection{Surface treatment of GrF reinforcement}

Though SMP epoxy easily wets GrF, interfacial spacing is observed in some regions of the composite. To fully harness in-plane stiffness of graphene sheets, the surface modification should be performed on GrF. The modification can be in the form of surface treatment such as low plasma treatment or creating more functionalized GrF.

\subsection{Optimizing GNP content in 3D printed SMP Epoxy Composites}

This research considers only 0.1 wt.\% GNP filler for the 3D printed SMP epoxy composite to examine GNP role in improving shape memory properties of epoxy. $0.1 \mathrm{wt} . \%$ 
GNP is considered because more weight percent GNP could lead to agglomeration and result in nozzle clogging (where the nozzle size is $400 \mu \mathrm{m}$ ). This could further lead to poor printing resolution. Also, lower GNP weight percent than $0.1 \mathrm{wt} \% \%$ might not produce the minimum mechanical and thermal reinforcement efficiency needed by SMP epoxy matrix. Thus, 3D printing of SMP epoxy-GNP composites with varying GNP contents would provide insights about the maximum GNP weight percent addition needed before clogging of the syringe nozzle. Optimized GNP content amount for shape memory and mechanical performance of epoxy would likewise be known.

\subsection{Control Process Parameters Optimization for 3D printing of SMP Epoxy-GNP Composite}

In addition to optimizing the content of graphene-based fillers, 3D printing process parameters should also be optimized. Appropriate process parameters are pivotal in obtaining excellent resolution 3D print of SMP epoxy composites. Though the printing parameters used in this study are based on our recent experiments and literature, a detailed parametric study should be performed to examine if the print resolution can be maximized. From the 3D printing of SMP epoxy, it has been observed that nozzle speed, bed plate temperature, print infill density, and layer height are the most important parameters to be considered for excellent resolution. A design of experiment containing an experimental matrix of these parameters would make the researcher make an informed decision about parameter values that would make up the excellent 3D print resolution of SMP epoxybased composites. 


\subsection{Modifications on SMP epoxy precursor and 3D Printing Instrumentation}

Challenge about more than one build layer of SMP epoxy, experienced in this study, should be addressed. Modification in the chemistry of SMP epoxy is needed to overcome the smudging issue of SMP epoxy precursor when used to print above one build layer. This would be important in using SMP epoxy for 3D printing of miniaturized components. Also, an external component which houses freeze sprayer is required. The freeze spray liquid is applied on thermal sensitive polymers like SMP epoxy to prevent smearing of the precursor.

\subsection{Fabrication of Kirigami-based SMP Epoxy Composites}

An interesting future work that is worth considering is mechanical functionalization

of thin sheets of SMP epoxy-graphene based system to create kirigami smart material. Kirigami design transforms a 2D material to 3D structure. Using kirigami concept, thin sheets of SMP epoxy-graphene composite be patterned or designed by a laser machine. This process transforms SMP epoxy-graphene based system to a multifunctional advanced composite, capable of shape recovery and be transformed into 3D configuration. Such a new generation of the advanced composite can find application in aerospace components, electronics (supercapacitors), and biomedical components. 


\section{REFERENCE}

[1] M. D. Hager, S. Bode, C. Weber, and U. S. Schubert, "Shape memory polymers: Past, present and future developments," Prog. Polym. Sci., vol. 49-50, pp. 3-33, 2015.

[2] R. Bogue, "Smart materials: A review of recent developments," Assem. Autom., vol. 32, no. 1, pp. 3-7, 2012.

[3] P. Schattling, F. D. Jochum, and P. Theato, "Multi-stimuli responsive polymers-the all-in-one talents," Polym. Chem., vol. 5, no. 1, pp. 25-36, 2014.

[4] Z. Q. Cao and G. J. Wang, "Multi-Stimuli-Responsive Polymer Materials: Particles, Films, and Bulk Gels," Chem. Rec., no. April, pp. 1398-1435, 2016.

[5] Y. J. Tan, J. Wu, H. Li, and B. C. K. Tee, "Self-Healing Electronic Materials for a Smart and Sustainable Future," ACS Appl. Mater. Interfaces, vol. 10, no. 18, pp. 15331-15345, 2018.

[6] M. S. Sarif Ullah Patwary, "Smart Textiles and Nano-Technology: A General Overview," J. Text. Sci. Eng., vol. 05, no. 01, pp. 1-7, 2015.

[7] D. Schaefer and W. M. Cheung, "Smart Packaging: Opportunities and Challenges," Procedia CIRP, vol. 72, pp. 1022-1027, 2018.

[8] I. L. Sokolov, V. R. Cherkasov, A. A. Tregubov, S. R. Buiucli, and M. P. Nikitin, "Smart materials on the way to theranostic nanorobots: Molecular machines and nanomotors, advanced biosensors, and intelligent vehicles for drug delivery," Biochim. Biophys. Acta - Gen. Subj., vol. 1861, no. 6, pp. 1530-1544, 2017.

[9] A. Yekane and S. Panchal, "Use of Smart Material made an Industrial Revolution in Development of Automobile," IOSR J. Comput. Eng., pp. 9-12, 2016.

[10] J. Leng, H. Lu, Y. Liu, W. M. Huang, and S. Du, "Shape-memory polymers - A class of novel smart materials," MRS Bull., vol. 34, no. 11, pp. 848-855, 2009.

[11] G. N and F. M, "Smart Materials and Structures: State of the Art and Applications," Nanotechnol. Appl., vol. 1, no. 2, pp. 1-5, 2018.

[12] B. M. Behl, M. Y. Razzaq, and A. Lendlein, "Multifunctional Shape-Memory Polymers," pp. 3388-3410, 2010.

[13] S. Rimdusit, M. Lohwerathama, K. Hemvichian, P. Kasemsiri, and I. Dueramae, "Shape memory polymers from benzoxazine-modified epoxy," Smart Mater. Struct., vol. 22, no. 7, 2013.

[14] A. B. Leonardi et al., "Shape memory epoxies based on networks with chemical and physical crosslinks," Eur. Polym. J., vol. 47, no. 3, pp. 362-369, 2011.

[15] B. I. A. Tli, F. A. G. Andhi, and G. R. E. G. K. Arst, "Thermomechanical Characterization of Shape Memory Polymers," vol. 20, no. January, 2009. 
[16] N. Zheng, G. Fang, Z. Cao, Q. Zhao, and T. Xie, "High strain epoxy shape memory polymer," Polym. Chem., vol. 6, no. 16, pp. 3046-3053, 2015.

[17] I. A. Rousseau, "Challenges of Shape Memory Polymers : A Review of the Progress Toward Overcoming SMP's Limitations," 2008.

[18] Changdeng Liu and P. T. Mather, "A shape memory polymer with improved shape recovery,” Mater. Res. Soc. Symp. Proc., vol. 855, no. 2004, pp. 1-6, 2005.

[19] X. Wu, Y. Liu, and J. Leng, "Investigation of mechanical behavior of epoxy shape memory polymers," Proc. SPIE, vol. 7289, no. 0, pp. 72890Z-72890Z-8, 2009.

[20] W. Chen and P. Fan, "Epoxy shape memory polymer ( SMP ): Material preparation , uniaxial tensile tests and dynamic mechanical analysis," no. July, 2017.

[21] F. Cao and S. C. Jana, "Nanoclay-tethered shape memory polyurethane nanocomposites," Polymer (Guildf)., vol. 48, no. 13, pp. 3790-3800, 2007.

[22] Y. Liu et al., "High Performance Shape Memory Epoxy/Carbon Nanotube Nanocomposites," 2016.

[23] A. Idowu, B. Boesl, and A. Agarwal, "3D graphene foam-reinforced polymer composites - A review," Carbon N. Y., vol. 135, pp. 52-71, 2018.

[24] C. Lee, X. Wei, J. W. Kysar, and J. Hone, "Measurement of the Elastic Properties and Intrinsic Strength of Monolayer Graphene," Science (80-. )., vol. 321, no. July, pp. 385-389, 2008.

[25] E. C. S. Transactions and T. E. Society, "Thermal Transport in Graphene Nanostructures: Experiments and Simulations Luis A. Jauregui,” vol. 28, no. 5, pp. 73-83, 2010.

[26] Z. U. Khan, A. Kausar, H. Ullah, and A. Badshah, "A review of graphene oxide, graphene buckypaper, and polymer / graphene composites: Properties and fabrication techniques," 2016.

[27] D. G. Papageorgiou, I. A. Kinloch, and R. J. Young, "Progress in Materials Science Mechanical properties of graphene and graphene-based nanocomposites," Prog. Mater. Sci., vol. 90, pp. 75-127, 2017.

[28] V. Balaji, K. Lau, D. Hui, and D. Bhattacharyya, "Graphene-based materials and their composites : A review on production , applications and product limitations," Compos. Part B, vol. 142, no. December 2017, pp. 200-220, 2018.

[29] J. Dai, G. Wang, L. Ma, and C. Wu, "SURFACE PROPERTIES OF GRAPHENE : RELATIONSHIP TO GRAPHENE-POLYMER COMPOSITES,” vol. 40, pp. 60$71,2015$.

[30] L. Tang, Y. Wan, D. Yan, Y. Pei, L. Zhao, and Y. Li, "The effect of graphene dispersion on the mechanical properties of graphene / epoxy composites," Carbon N. Y., vol. 60, pp. 16-27, 2013. 
[31] W. Zheng, B. Shen, and W. Zhai, "Surface Functionalization of Graphene with Polymers for Enhanced Properties," 2013.

[32] P. G. Sponges, "Highly Electrically Conductive Nanocomposites Based on Polymer-Infused Graphene Sponges,” pp. 1-6, 2014.

[33] R. Raj, S. C. Maroo, and E. N. Wang, "Wettability of graphene," Nano Lett., vol. 13, no. 4, pp. 1509-1515, 2013.

[34] A. Nieto, B. Boesl, and A. Agarwal, "Multi-scale intrinsic deformation mechanisms of 3D graphene foam," Carbon N. Y., vol. 85, pp. 299-308, 2015.

[35] F. Wypych and K. G. Satyanarayana, "Functionalization of single layers and nanofibers: A new strategy to produce polymer nanocomposites with optimized properties," J. Colloid Interface Sci., vol. 285, no. 2, pp. 532-543, 2005.

[36] O. Breuer and U. Sundararaj, "Big returns from small fibers: A review of polymer/carbon nanotube composites," Polym. Compos., vol. 25, no. 6, pp. 630645, 2004.

[37] Z. M. Huang, Y. Z. Zhang, M. Kotaki, and S. Ramakrishna, "A review on polymer nanofibers by electrospinning and their applications in nanocomposites," Compos. Sci. Technol., vol. 63, no. 15, pp. 2223-2253, 2000.

[38] R. Jan, A. Habib, and H. Y. Abbasi, "High Aspect Ratio Graphene Nanosheets Cause a Very Low Percolation Threshold for Polymer Nanocomposites," vol. 129, no. 4, pp. 478-481, 2016.

[39] E. Beyou, S. Akbar, P. Chaumont, and P. Cassagnau, "Polymer Nanocomposites Containing Functionalised Multiwalled Carbon NanoTubes : a Particular Attention to Polyolefin Based Materials," 1991.

[40] A. Peigney, C. Laurent, E. Flahaut, R. R. Bacsa, and A. Rousset, "Specific surface area of carbon nanotubes and bundles of carbon nanotubes," Carbon N. Y., vol. 39, no. 4, pp. 507-514, 2001.

[41] B. Y. Zhu et al., "Graphene and Graphene Oxide: Synthesis , Properties , and Applications," pp. 3906-3924, 2010.

[42] K. I. M. E. T. Al, “Activated Graphene-Based Carbons as Supercapacitor Electrodes with," no. 8, pp. 6899-6905, 2013.

[43] D. R. Paul and L. M. Robeson, "Polymer nanotechnology: Nanocomposites," Polym. with aligned carbon Nanotub. Act. Compos. Mater., vol. 49, no. 15, pp. 3187-3204, 2008.

[44] B. Li and W. H. Zhong, "Review on polymer/graphite nanoplatelet nanocomposites," J. Mater. Sci., vol. 46, no. 17, pp. 5595-5614, 2011.

[45] J. Liang et al., "Electromagnetic interference shielding of graphene/epoxy composites," Carbon N. Y., vol. 47, no. 3, pp. 922-925, 2009. 
[46] H. Hu et al., "Preparation and properties of graphene nanosheets-polystyrene nanocomposites via in situ emulsion polymerization," Chem. Phys. Lett., vol. 484, no. 4-6, pp. 247-253, 2010.

[47] H. Bin Zhang et al., "Electrically conductive polyethylene terephthalate/graphene nanocomposites prepared by melt compounding," Polymer (Guildf)., vol. 51, no. 5, pp. 1191-1196, 2010.

[48] G. Chen, Y. Liu, F. Liu, and X. Zhang, "Applied Surface Science Fabrication of three-dimensional graphene foam with high electrical conductivity and large adsorption capability," Appl. Surf. Sci., vol. 311, pp. 808-815, 2014.

[49] M. T. Pettes, H. Ji, and L. Shi, "Thermal Transport in Three-Dimensional Foam Architectures of Few- Layer Graphene and Ultrathin Graphite," 2012.

[50] K. M. Yocham et al., "Mechanical Properties of Graphene Foam and Graphene Foam - Tissue Composites," vol. 1800166, pp. 1-9, 2018.

[51] H. Bi et al., "Spongy graphene as a highly efficient and recyclable sorbent for oils and organic solvents," Adv. Funct. Mater., vol. 22, no. 21, pp. 4421-4425, 2012.

[52] S. Mao, K. Yu, J. Chang, D. a Steeber, L. E. Ocola, and J. Chen, "Direct growth of vertically-oriented graphene for field-effect transistor biosensor.," Sci. Rep., vol. 3, p. 1696, 2013.

[53] K. Yu, P. Wang, G. Lu, K. Chen, Z. Bo, and J. Chen, "Patterning Vertically Oriented Graphene Sheets for Nanodevice," pp. 537-542, 2011.

[54] Y. Chen, "properties and applications," pp. 40-53, 2015.

[55] V. A. Online, Y. Li, K. Sheng, W. Yuan, and G. Shi, "A high-performance flexible fibre-shaped electrochemical capacitor based on electrochemically reduced graphene oxide $\uparrow, "$ pp. 291-293, 2013.

[56] X. Wang et al., "Three-dimensional strutted graphene grown by substrate-free sugar blowing for high-power-density supercapacitors," Nat. Commun., vol. 4, no. May, pp. 1-8, 2013.

[57] S. Process, H. Cong, X. Ren, P. Wang, and S. Yu, "Macroscopic Multifunctional Graphene-Based Hydrogels and Aerogels by a Metal Ion Induced," no. 3, pp. 26932703, 2012.

[58] X. Huang et al., "Functional nanoporous graphene foams with controlled pore sizes," Adv. Mater., vol. 24, no. 32, pp. 4419-4423, 2012.

[59] J. Lee, S. Kim, J. Yoon, J. Jang, and L. E. E. E. T. Al, "Chemical Vapor Deposition of Mesoporous Graphene Nanoballs for Supercapacitor," no. 7, pp. 6047-6055, 2013. 
[60] S. Ye and J. Feng, "Self-Assembled Three-Dimensional Hierarchical Graphene/ Polypyrrole Nanotube Hybrid Aerogel and Its Application for Supercapacitors," 2014.

[61] X. Cao, Z. Yin, and H. Zhang, "Environmental Science," Energy Environ. Sci., no. 7, pp. 1850-1865, 2014.

[62] V. Chabot, D. Higgins, A. Yu, and X. Xiao, "Environmental Science material synthesis and applications to energy and," RSC, 2014.

[63] H. Zhan et al., "Direct fabrication of 3D graphene on nanoporous anodic alumina by plasma-enhanced chemical vapor deposition," Sci. Rep., vol. 6, no. August 2015, p. 19822, 2016.

[64] M. A. Worsley, P. J. Pauzauskie, T. Y. Olson, J. Biener, J. H. Satcher, and T. F. Baumann, "Synthesis of Graphene Aerogel with High Electrical Conductivity," pp. 14067-14069, 2010.

[65] J. H. Kim et al., "3D printing of reduced graphene oxide nanowires," Adv. Mater., vol. 27, no. 1, pp. 157-161, 2015.

[66] M. Yu et al., "Building three-dimensional graphene frameworks for energy storage and catalysis," Adv. Funct. Mater., vol. 25, no. 2, pp. 324-330, 2015.

[67] Z. Chen, W. Ren, L. Gao, B. Liu, S. Pei, and H.-M. Cheng, "Three-dimensional flexible and conductive interconnected graphene networks grown by chemical vapour deposition.," Nat. Mater., vol. 10, no. 6, pp. 424-428, 2011.

[68] A. Reina et al., "Large Area , Few-Layer Graphene Films on Arbitrary Substrates by Chemical Vapor Deposition 2009," pp. 1-6, 2009.

[69] Q. Yu et al., "Graphene segregated on Ni surfaces and transferred to insulators Graphene segregated on Ni surfaces and transferred to insulators," vol. 113103, no. 2008, pp. 1-4, 2011.

[70] P. Nautiyal, B. Boesl, and A. Agarwal, "Harnessing Three Dimensional Anatomy of Graphene Foam to Induce Superior Damping in Hierarchical Polyimide Nanostructures," Small, vol. 13, no. 10, 2017.

[71] J. Jia, X. Sun, X. Lin, X. Shen, Y. W. Mai, and J. K. Kim, "Exceptional electrical conductivity and fracture resistance of 3D interconnected graphene foam/epoxy composites," ACS Appl. Mater. Interfaces, vol. 8, no. 9, pp. 39717-39727, 2017.

[72] P. Nautiyal, B. Boesl, and A. Agarwal, "The mechanics of energy dissipation in a three-dimensional graphene foam with macroporous architecture," Carbon N. Y., vol. 132, pp. 59-64, 2018.

[73] D. Pan, C. Wang, T. Wang, and Y. Yao, "Graphene Foam : Uniaxial Tension Behavior and Fracture Mode Based on a Mesoscopic Model," ACS Nano, no. 11, pp. 8988-8997, 2017. 
[74] Z. Qin, G. S. Jung, M. J. Kang, and M. J. Buehler, "The mechanics and design of a lightweight three-dimensional graphene assembly," Sci. Adv., vol. 3, pp. 1-9, 2017.

[75] E. Singh et al., "Superhydrophobic graphene foams," Small, vol. 9, no. 1, pp. 7580, 2013.

[76] D. a. C. Brownson et al., "Freestanding three-dimensional graphene foam gives rise to beneficial electrochemical signatures within non-aqueous media," J. Mater. Chem. A, vol. 1, no. 19, p. 5962, 2013.

[77] Y. Zhang et al., "Broadband and tunable high-performance microwave absorption of an ultralight and highly compressible graphene foam," Adv. Mater., vol. 27, no. 12, pp. 2049-2053, 2015.

[78] L. Embrey, P. Nautiyal, A. Loganathan, A. Idowu, B. Boesl, and A. Agarwal, "Three-Dimensional Graphene Foam Induces Multifunctionality in Epoxy Nanocomposites by Simultaneous Improvement in Mechanical , Thermal , and Electrical Properties," ACS Appl. Mater. Interfaces, no. 9, pp. 39717-39727, 2017.

[79] S. R. Bakshi, J. E. Tercero, and A. Agarwal, "Synthesis and characterization of multiwalled carbon nanotube reinforced ultra high molecular weight polyethylene composite by electrostatic spraying technique," Compos. Part A Appl. Sci. Manuf., vol. 38, no. 12, pp. 2493-2499, 2007.

[80] Y. A. Samad, Y. Li, S. M. Alhassan, and K. Liao, "Novel Graphene Foam Composite with Adjustable Sensitivity for Sensor Applications," ACS Appl. Mater. Interfaces, 2015.

[81] C. Zhang, B. Boesl, and A. Agarwal, "Three-Dimensional Graphene Foam Polymer Composite with Superior Deicing E ffi ciency and Strength,” 2018.

[82] Q. Zhang, X. Xu, H. Li, G. Xiong, H. Hu, and T. S. Fisher, "Mechanically robust honeycomb graphene aerogel multifunctional polymer composites," Carbon N. Y., vol. 93, pp. 659-670, 2015.

[83] S. K. Reddy et al., "Highly compressible behavior of polymer mediated threedimensional network of graphene foam," RSC Adv., vol. 4, no. 91, pp. 50074-50080, 2014.

[84] J. Jia, X. Du, C. Chen, X. Sun, Y. W. Mai, and J. K. Kim, “3D network graphene interlayer for excellent interlaminar toughness and strength in fiber reinforced composites," Carbon N. Y., vol. 95, pp. 978-986, 2015.

[85] Y. Qiu, J. Liu, Y. Lu, R. Zhang, W. Cao, and P. Hu, "Hierarchical Assembly of Tungsten Spheres and Epoxy Composites in Three-Dimensional Graphene Foam and Its Enhanced Acoustic Performance as a Backing Material," ACS, vol. 8, no. 28, pp. 18496-18504, 2016. 
[86] A. Idowu, P. Nautiyal, L. Fontoura, A. Loganathan, and B. Boesl, "Multi-Scale Damping of Graphene Foam-Based Polyurethane Composites Synthesized by Electrostatic Spraying," Polym. Chem., pp. 1-9, 2018.

[87] A. Nieto, R. Dua, C. Zhang, B. Boesl, and S. Ramaswamy, "Three Dimensional Graphene Foam / Polymer Hybrid as a High Strength Biocompatible Scaffold," pp. 3916-3924, 2015.

[88] J. Li et al., "Highly Stretchable and Sensitive Strain Sensor Based on Facilely Prepared Three-Dimensional Graphene Foam Composite," ACS Appl. Mater. Interfaces, vol. 8, no. 29, pp. 18954-18961, 2016.

[89] Y. R. Jeong, H. Park, S. W. Jin, S. Y. Hong, S. S. Lee, and J. S. Ha, "Highly Stretchable and Sensitive Strain Sensors Using Fragmentized Graphene Foam," Adv. Funct. Mater., vol. 25, no. 27, pp. 4228-4236, 2015.

[90] Y.-H. Zhao, Y.-F. Zhang, S.-L. Bai, and X.-W. Yuan, "Carbon fibre/graphene foam/polymer composites with enhanced mechanical and thermal properties," Compos. Part B Eng., vol. 94, pp. 102-108, 2016.

[91] G. Chen, Y. Liu, F. Liu, and X. Zhang, "Fabrication of three-dimensional graphene foam with high electrical conductivity and large adsorption capability," Appl. Surf. Sci., vol. 311, pp. 808-815, 2014.

[92] X. Sun, X. Liu, X. Shen, Y. Wu, Z. Wang, and J. K. Kim, "Graphene foam/carbon nanotube/poly(dimethyl siloxane) composites for exceptional microwave shielding," Compos. Part A Appl. Sci. Manuf., vol. 85, pp. 199-206, 2015.

[93] Y. S. Jun et al., "Highly conductive interconnected graphene foam based polymer composite," Carbon N. Y., vol. 95, pp. 653-658, 2015.

[94] J. K. Wang et al., "Polymer-enriched 3D graphene foams for biomedical applications," ACS Appl. Mater. Interfaces, vol. 7, no. 15, pp. 8275-8283, 2015.

[95] S. . Chabi, C. . Peng, Z. . Yang, Y. . Xia, and Y. . Zhu, "Three dimensional (3D) flexible graphene foam/polypyrrole composite: Towards highly efficient supercapacitors," RSC Adv., vol. 5, no. 6, pp. 3999-4008, 2015.

[96] M. Loeblein et al., "3D Graphene-Infused Polyimide with Enhanced Electrothermal Performance for Long-Term Flexible Space Applications," Small, vol. 11, no. 48, pp. 6425-6434, 2015.

[97] J. Pedrós et al., "Polyaniline nanofiber sponge filled graphene foam as high gravimetric and volumetric capacitance electrode," J. Power Sources, vol. 317, pp. 35-42, 2016.

[98] H. Fukushima, L. T. Drzal, B. P. Rook, and M. J. Rich, "Thermal conductivity of exfoliated graphite nanocomposites," J. Therm. Anal. Calorim., vol. 85, no. 1, pp. 235-238, 2006. 
[99] O. C. Hung, M.-T, "Heat conduction in graphite-nanoplatelet- reinforced polymer nanocomposites," Appl. Phys. Lett., vol. 023117, no. June 2006, 2012.

[100] M. T. Pettes, H. Ji, and L. Shi, "Thermal Transport in Three-Dimensional Foam Architectures of Few- Layer Graphene and Ultrathin Graphite,” 2012.

[101] M. Li, Y. Sun, H. Xiao, X. Hu, and Y. Yue, "High temperature dependence of thermal transport in graphene foam.," Nanotechnology, vol. 26, no. 10, p. 105703, 2015.

[102] X. Zhang et al., "Exceptional thermal interface properties of a three-dimensional graphene foam," Carbon N. Y., vol. 66, pp. 201-209, 2014.

[103] K. Zhang, M. M. F. Yuen, N. Wang, J. Y. Miao, D. G. W. Xiao, and H. B. Fan, "Thermal interface material with aligned CNT and its application in HB-LED packaging," Proc. - Electron. Components Technol. Conf., vol. 2006, pp. 177-182, 2006.

[104] Y.-H. Zhao, Z.-K. Wu, and S.-L. Bai, "Thermal resistance measurement of 3D graphene foam/polymer composite by laser flash analysis," Int. J. Heat Mass Transf., vol. 101, pp. 470-475, 2016.

[105] X. Li, L. Shao, N. Song, L. Shi, and P. Ding, "Enhanced thermal-conductive and anti-dripping properties of polyamide composites by 3D graphene structures at low filler content," Compos. Part A Appl. Sci. Manuf., vol. 88, pp. 1653-1655, 2014.

[106] Y. H. Zhao, Y. F. Zhang, and S. L. Bai, "High thermal conductivity of flexible polymer composites due to synergistic effect of multilayer graphene flakes and graphene foam," Compos. Part A Appl. Sci. Manuf., vol. 85, pp. 148-155, 2016.

[107] Y. H. Zhao, Y. F. Zhang, Z. K. Wu, and S. L. Bai, "Synergic enhancement of thermal properties of polymer composites by graphene foam and carbon black," Compos. Part B Eng., vol. 84, pp. 52-58, 2016.

[108] J. Liu, T. Wang, J. Wang, and E. Wang, "Mussel-inspired biopolymer modified 3D graphene foam for enzyme immobilization and high performance biosensor," Electrochim. Acta, vol. 161, pp. 17-22, 2015.

[109] and S.-L. B. Qin-Ying Wang,\# Yun-Hong Zhao,\# Zhen-Kun Wu, "Exploring the Corrosion Behavior of Polymer- based 3D Graphene-Nickel Foam Composites," Chem. leters, no. August, pp. 1653-1655, 2014.

[110] M. Behl and A. Lendlein, "Shape-memory polymers," Mater. Today, vol. 10, no. 4, pp. 20-28, 2007.

[111] A. M. Schmidt, "Electromagnetic Activation of Shape Memory Polymer Networks Containing Magnetic Nanoparticles a,” pp. 1168-1172, 2006.

[112] J. M. Chem, "Spatial and temporal control of shape memory polymers and simultaneous drug release using high intensity focused ultrasound $\uparrow, "$ pp. 76927696, 2012. 
[113] A. Lendlein and S. Kelch, "Shape-Memory Effect From shape ... ... to permanent shape."

[114] J. W. Cho, J. W. Kim, Y. C. Jung, and N. S. Goo, "Electroactive Shape-Memory Polyurethane Composites Incorporating Carbon Nanotubes,” pp. 412-416, 2005.

[115] T. M. Conjugated and R. Langer, "Light-induced shape-memory polymers "," vol. 434, no. April, pp. 695-697, 2005.

[116] W. W. and W. L. Tao Bai, $\$$ Yanjiao Han, + Peng Zhang, "Soft Matter," Soft Matter, no. 8, pp. 6846-6852, 2012.

[117] F. Xie, L. Huang, J. Leng, and Y. Liu, "Thermoset shape memory polymers and their composites," J. Intell. Mater. Syst. Struct., vol. 27, no. 18, pp. 2433-2455, 2016.

[118] T. Xie and I. A. Rousseau, "Facile tailoring of thermal transition temperatures of epoxy shape memory polymers," Polymer (Guildf)., vol. 50, no. 8, pp. 1852-1856, 2009.

[119] F. Pilate, A. Toncheva, P. Dubois, and J. Raquez, "Shape-memory polymers for multiple applications in the materials world," Eur. Polym. J., vol. 80, pp. 268-294, 2016.

[120] T. Composites, "Review of Progress in Shape Memory Epoxies and Their Composites," Polymers (Basel)., pp. 1-38, 2018.

[121] L. Min, X. Feng, Y. Feng, and X. Jun, "Shape Memory Effect and Mechanical Properties of Graphene / Epoxy Composites 1,” vol. 56, no. 5, pp. 640-645, 2014.

[122] E. Pop, V. Varshney, and A. K. Roy, "Thermal properties of graphene: Fundamentals and applications," MRS Bull., vol. 37, no. December, pp. 1273-1281, 2012.

[123] Z. Sun, Z. Li, C. Huang, Y. Zhao, H. Zhang, and R. Tao, "Ultrasonication-assisted uniform decoration of carbon nanotubes by various particles with controlled size and loading," vol. 9, pp. 3-11, 2011.

[124] E. Wang et al., "A novel reduced graphene oxide / epoxy sandwich structure composite film with thermo-, electro- and light-responsive shape memory effect," Mater. Lett., vol. 238, pp. 54-57, 2019.

[125] A. P. Chuanjin Huang, Jingsong Peng, Yiren Cheng, Qian Zhao, Yi Du, Shixue Dou, "Materials Chemistry A," Mater. Chem. A, 2019.

[126] Z. Yu, Z. Wang, H. Li, J. Teng, and L. Xu, "Shape Memory Epoxy Polymer ( SMEP ) Composite Mechanical Properties Enhanced by Introducing Graphene Oxide ( GO ) into the Matrix," no. 1, 2019. 
[127] W. Wang, D. Liu, Y. Liu, J. Leng, and D. Bhattacharyya, "Electrical actuation properties of reduced graphene oxide paper / epoxy-based shape memory composites," Compos. Sci. Technol., vol. 106, pp. 20-24, 2015.

[128] L. Chen and Y. Liu, "Microwave responsive epoxy nanocomposites reinforced by carbon nanomaterials of different dimensions," vol. 45676, pp. 1-10, 2018.

[129] X. Liu, H. Li, Q. Zeng, Y. Zhang, H. Kang, and H. Duan, "Electro-active shape memory composites enhanced by fl exible carbon nanotube / graphene aerogels," $J$. Mater. Chem. A Mater. energy Sustain., vol. 00, pp. 1-9, 2015.

[130] E. Wang et al., "E ff ect of graphene oxide-carbon nanotube hybrid fi ller on the mechanical property and thermal response speed of shape memory epoxy composites," Compos. Sci. Technol., vol. 169, no. November 2018, pp. 209-216, 2019.

[131] Y. Wang, W. Tian, J. Xie, and Y. Liu, "Thermoelectric responsive shape memory graphene/hydro-epoxy composites for actuators," Micromachines, vol. 7, no. 8, 2016.

[132] X. Xiao and Y. Cheng, "Self-healable graphene polymer composites †," pp. 35083514, 2010.

[133] B. Application and A. Kausar, "Advances in Polymer / Graphene Nanocomposite for," pp. 23-28, 2018.

[134] H. Gao, J. Li, F. Zhang, Y. Liu, and J. Leng, "The research status and challenges of shape memory polymer-based flexible electronics," Mater. Horizons, vol. 6, no. 5, pp. 931-944, 2019.

[135] J. Li, Q. Duan, E. Zhang, and J. Wang, "Applications of shape memory polymers in kinetic buildings," Adv. Mater. Sci. Eng., vol. 2018, no. Figure 2, 2018.

[136] R. Liu et al., "Shape Memory Polymers for Body Motion Energy Harvesting and Self-Powered Mechanosensing," Adv. Mater., vol. 30, no. 8, pp. 1-8, 2018.

[137] S. L. Buffington, J. E. Paul, M. M. Ali, M. M. Macios, P. T. Mather, and J. H. Henderson, "Enzymatically triggered shape memory polymers," Acta Biomater., vol. 84, pp. 88-97, 2019.

[138] A. Khosrozadeh, G. Singh, Q. Wang, G. Luo, and M. Xing, "Supercapacitor with extraordinary cycling stability and high rate from nano-architectured polyaniline/graphene on Janus nanofibrous film with shape memory," J. Mater. Chem. A, vol. 6, no. 42, pp. 21064-21077, 2018.

[139] M. Shtein, R. Nadiv, M. Buzaglo, and O. Regev, "Graphene-Based Hybrid Composites for Efficient Thermal Management of Electronic Devices," ACS Appl. Mater. Interfaces, vol. 7, no. 42, pp. 23725-23730, 2015.

[140] XG Sciences Inc, "xGnP ® Graphene Nanoplatelets Carbon Nanoparticles with Multifunctional Capability." 
[141] W. Choi, I. Lahiri, R. Seelaboyina, and Y. S. Kang, "Synthesis of graphene and its applications: A review," Crit. Rev. Solid State Mater. Sci., vol. 35, no. 1, pp. 52-71, 2010.

[142] G. S. Bumbrah and R. M. Sharma, "Raman spectroscopy - Basic principle, instrumentation and selected applications for the characterization of drugs of abuse," Egypt. J. Forensic Sci., vol. 6, no. 3, pp. 209-215, 2016.

[143] Y. Liu, H. Sun, H. Tan, and X. Du, "Modified shape memory epoxy resin composites by blending activity polyurethane," J. Appl. Polym. Sci., vol. 127, no. 4, pp. 3152-3158, 2013.

[144] Z. Jin et al., "Click chemistry on solution-dispersed graphene and monolayer CVD graphene," Chem. Mater., vol. 23, no. 14, pp. 3362-3370, 2011.

[145] B. J. Emsley and S. H. Bonds, "Very Strong Hydrogen Bonding," Chem. Soc. Rev., vol. 9, pp. 91-124, 1980.

[146] K. H. Liao, S. Aoyama, A. A. Abdala, and C. Macosko, "Does graphene change T g of nanocomposites?," Macromolecules, vol. 47, no. 23, pp. 8311-8319, 2014.

[147] J. Xu and J. Song, "Thermal Responsive Shape Memory Polymers for Biomedical Applications," Biomed. Eng. - Front. Challenges, pp. 125-142, 2011.

[148] J. Leng, X. Lan, Y. Liu, and S. Du, "Shape-memory polymers and their composites: Stimulus methods and applications," Prog. Mater. Sci., vol. 56, no. 7, pp. 10771135, 2011.

[149] Y. Zhou and W. M. Huang, "Shape Memory Effect in Polymeric Materials: Mechanisms and Optimization," Procedia IUTAM, vol. 12, pp. 83-92, 2015.

[150] W. Wang, D. Liu, Y. Liu, J. Leng, and D. Bhattacharyya, "Electrical actuation properties of reduced graphene oxide paper/epoxy-based shape memory composites," Compos. Sci. Technol., vol. 106, pp. 20-24, 2015.

[151] P. Zhang et al., "A Theoretical Review on Interfacial Thermal Transport at the Nanoscale," Small, vol. 1702769, p. 1702769, 2017.

[152] D. Roylance, "Mechanical Properties of Materials," 2017.

[153] D. Zhang, M. He, S. Qin, and J. Yu, "Effect of fiber length and dispersion on properties of long glass fiber reinforced thermoplastic composites based on poly(butylene terephthalate)," RSC Adv., vol. 7, no. 25, pp. 15439-15454, 2017.

[154] K. Menard, Dynamic Mechanical Anaylsis. .

[155] R. M. Barron, T. H. K. Barron, P. M. Mummery, and M. Sharkey, "Thermal expansion and Grüneisen functions of polymer crystal models: 1. Central forces," Can. J. Chem., vol. 66, no. 4, pp. 718-724, 1988. 
[156] X. Wang, M. Jiang, Z. Zhou, J. Gou, and D. Hui, “3D printing of polymer matrix composites: A review and prospective," Compos. Part B Eng., vol. 110, pp. 442458, 2017.

[157] J. Bustillos, D. Montero, P. Nautiyal, A. Loganathan, and B. Boesl, "Integration of Graphene in Poly ( Lactic ) Acid by 3D Printing to Develop Creep and WearResistant Hierarchical Nanocomposites," 2017.

[158] A. Nieto, D. Lahiri, and A. Agarwal, "Synthesis and properties of bulk graphene nanoplatelets consolidated by spark plasma sintering," Carbon N. Y., vol. 50, no. 11, pp. 4068-4077, 2012.

[159] H. Vašková and V. Křesálek, "Quasi real-time monitoring of epoxy resin crosslinking via Raman microscopy," Int. J. Math. Model. Methods Appl. Sci., vol. 5, no. 7, pp. 1197-1204, 2011.

[160] W. Pu, F. Jiang, B. Wei, Y. Tang, and Y. He, "Influences of structure and multiintermolecular forces on rheological and oil displacement properties of polymer solutions in the presence of $\mathrm{Ca} 2+/ \mathrm{Mg} 2+$, , $R S C A d v$., vol. 7, no. 8, pp. 4430-4436, 2017.

[161] D. G. Papageorgiou, I. A. Kinloch, and R. J. Young, "Mechanical properties of graphene and graphene-based nanocomposites," Prog. Mater. Sci., vol. 90, pp. 75127, 2017.

[162] Embrey, Leslie, "Three-Dimensional Graphene Foam Reinforced Epoxy Composites" (2017). FIU Electronic Theses and Dissertations. https://digitalcommons.fiu.edu/etd/3128. 
VITA

\section{ADEYINKA TAIWO IDOWU}

Email address: aidow002@fiu.edu; yinksid@gmail.com

Phone number: +17864684721

EDUCATION

Sept 2005 - Nov 2009 Bachelors of Science (B.S.) in Metallurgical and Materials Engineering, University of Lagos, Akoka, Lagos, Nigeria

Jan 2012 - May $2013 \quad$ Masters of Science (M.S.) in Materials Science and Engineering, African University of Science and Technology, FCT Abuja, Nigeria

Aug 2016 - Dec 2019 Doctoral Candidate (Ph.D) in Materials Engineering, Florida International University, Miami, USA

\section{PUBLICATIONS AND PRESENTATIONS}

1. A. Idowu, P. Nautiyal, J. Bustillos, B. Boesl, A. Agarwal. Shape Memory-Based SelfHealing Polymer Composite Reinforced with Graphene Foam, US Patent App. 16/421,562, June 2019.

2. A. Idowu, P. Nautiyal, B. Boesl, A. Agarwal. Multi-scale Mechanical Behavior of Three-dimensional Graphene Foam-based Shape Memory Epoxy Composites. Adeyinka Idowu, The Minerals, Metals and Materials (TMS) Conference, San Antonio, Texas, USA, March 10th-14 ${ }^{\text {th }}, 2019$.

3. A. Idowu, P. Nautiyal, L. Fontoura, A. Loganathan, and B. Boesl, "Multi-Scale Damping of Graphene Foam-Based Polyurethane Composites Synthesized by Electrostatic Spraying," Polym. Chem., pp. 1-9, 2018.

4. A. Idowu, B. Boesl, and A. Agarwal, "3D graphene foam-reinforced polymer composites - A review," Carbon N. Y., vol. 135, pp. 52-71, 2018.

5. L. Embrey, P. Nautiyal, A. Loganathan, A. Idowu, B. Boesl, and A. Agarwal, "ThreeDimensional Graphene Foam Induces Multifunctionality in Epoxy Nanocomposites by Simultaneous Improvement in Mechanical, Thermal, and Electrical Properties," ACS Appl. Mater. Interfaces, no. 9, pp. 39717-39727, 2017. 
6. Funsho Olaitan Kolawole, Sharafadeen Adetunji Adeniji, Adeyinka Idowu, Tunji Adetayo Owoseni, Odet Fayen Ngasoh, Wilson Oluwole Soboyejo, "Corrugated Laterite Based Ceramic Roof Tile Stabilized with Cement," IJET;4(3) 145 - 149.

7. S. O. Adeosun, O. I. Sekunowo, O. P. Gbenebor, W. A. Ayoola, A. O. Odunade, A. Idowu, "Mechanical Properties Enhancement of 66/34Mg-Alloy for Medical Application," World Academy of Science, Engineering and Technology International Journal of Medical, Health, Biomedical, Bioengineering and Pharmaceutical Engineering Vol:8, No:2, 2014.

8. A. Idowu, B. Boesl, A. Agarwal, (2017): "Multiscale Damping Behavior of Graphene Foam Reinforced Polyurethane Composites". Materials Science and Technology Conference and Exhibition, MS\&T 17, Pennsylvania, Pittsburg, USA.

9. A. Idowu and O. Ige, "Comparison of corrosion behavior of welded zone and parent metal of low carbon steel pipe (X65) in Acidic and Alkaline Environments", $8^{\text {th }}$ International AMRS conference in December 2015, Ghana.

10. A. Idowu and W. Soboyejo, "Effect of cut-back bitumen coatings on the corrosion of low carbon steels (API 5L X65)". $7^{\text {th }}$ Biennial International Conference of the Africa Materials Research Society (AMRS), Addis Ababa, Ethiopia, December 2013. 